\title{
Development of a Simulation and Optimization Framework for Improved Aerodynamic Performance of R/C Helicopter Rotor Blades
}

\author{
by \\ Jonathan Wiebe \\ B.Eng.
}

\author{
A thesis submitted to the \\ Faculty of Graduate and Postdoctoral Affairs \\ in partial fulfillment of the requirements for the degree of \\ Master of Applied Science \\ in \\ Aerospace Engineering \\ Ottawa-Carleton Institute for Mechanical and Aerospace Engineering \\ Department of Mechanical and Aerospace Engineering \\ Carleton University \\ Ottawa, Ontario \\ January, 2015 \\ Copyright (C) 2015 \\ Jonathan Wiebe
}


The undersigned hereby recommend to the

Faculty of Graduate and Postdoctoral Affairs acceptance of the thesis

\title{
Development of a Simulation and Optimization Framework for Improved Aerodynamic Performance of R/C Helicopter Rotor Blades
}

\author{
submitted by Jonathan Wiebe, B.Eng. \\ in partial fulfillment of the requirements for the degree of \\ Master of Applied Science \\ in \\ Aerospace Engineering
}

D. Feszty, Supervisor

M. I. Yaras, Department Chair

Carleton University

January, 2015 


\section{Abstract}

To improve the performance characteristics of small unmanned rotorcraft systems based on commercially available radio controlled helicopter components the simulation and optimization framework Qoptr was developed. The framework's simulation modules model main rotor performance in hover using blade element momentum theory (BEMT) and in forward flight conditions employing a blade element theory (BET) approach. The forward flight module incorporates empirical induced inflow models and rigid blade motion.

Two software packages based on viscous-inviscid interaction methods were evaluated on their ability to generate the low Reynolds number 2D aerodynamic airfoil performance coefficients required by the simulation modules at conditions applicable to large radio controlled helicopters.

The Qoptr hover module was integrated into an optimization scheme using an algorithm from the MATLAB Optimization Toolbox. Starting from a rotor using typical commercial $\mathrm{r} / \mathrm{c}$ rotor blades the optimization raised the rotor figure of merit from 0.56 to 0.70 by adjusting rotor speed, solidity and the spanwise distributions of blade pitch and chord length. 
To my parents: Abram and Larissa. 


\section{Acknowledgements}

I would like to thank God for his continued provision throughout my life. Thank you also to my parents and siblings for your love and support. I cherish the times we get to spend together and wouldn't be the person I am today, if it weren't for you. I need to acknowledge my uncle Paul, who encouraged me to go for my dreams even if that meant moving away from home.

Thank you to the professors and other staff at the mechanical and aerospace engineering department and my coop colleagues for providing challenging and interesting learning environments. Thank you to J. Ricciardi for introducing me to the vast field of helicopter engineering. I am grateful to my fellow students and friends at the Formula SAE teams over the years for sharing many eventful days and nights with me working on various assignments, projects or contraptions.

Finally, I would like to extend my appreciation to Prof. D. Feszty for coordinating this exciting project with our partners R. Gaziano and ING Robotic Aviation. Thank you for your technical guidance, encouragement and the professional development opportunities along the way.

This work was financially supported by the Natural Sciences and Engineering Research Council of Canada (NSERC) in collaboration with ING Robotic Aviation Inc. 


\section{Table of Contents}

Abstract

Acknowledgements $\quad$ iv

Table of Contents $\quad$ V

List of Tables $\quad$ viii

List of Figures $\quad$ ix

Nomenclature $\quad$ xii

1 Introduction 1

1.1 Small Rotorcraft UAS Operation . . . . . . . . . . . . . . 1

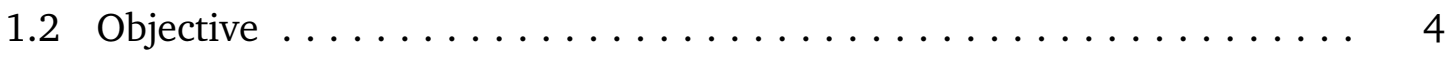

1.3 Organization of Thesis $\ldots \ldots \ldots \ldots \ldots \ldots \ldots \ldots \ldots \ldots \ldots \ldots \ldots \ldots \ldots \ldots$

2 Literature Review and Theory Background 6

2.1 Review of Small Rotor Performance Studies . . . . . . . . . . . . 6

2.2 Aerodynamic Performance Analysis Methods for Helicopter Rotors . . . 8

2.2.1 Momentum Theory $\ldots \ldots \ldots \ldots \ldots \ldots \ldots \ldots$

2.2.2 Blade Element Theory $\ldots \ldots \ldots \ldots \ldots \ldots \ldots \ldots$ 
2.2.3 Modern Rotorcraft Analysis Tools . . . . . . . . . . . . . . 24

2.2.4 Selection of Methodology $\ldots \ldots \ldots \ldots \ldots \ldots \ldots \ldots \ldots .25$

2.3 Airfoil Data Considerations $\ldots \ldots \ldots \ldots \ldots \ldots \ldots \ldots \ldots \ldots$

2.3.1 Airfoil Aerodynamic Characteristics............... 26

2.3.2 Representation of Static Airfoil Data . . . . . . . . . . . 30

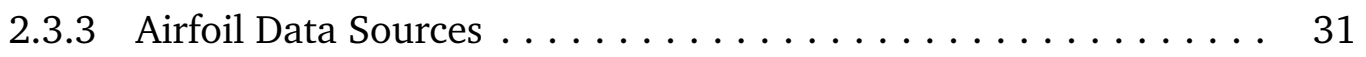

2.4 Optimization Applied to Rotorcraft Design . . . . . . . . . . . . . 34

2.4.1 Optimization Methods . . . . . . . . . . . . . . . . . 35

2.4.2 Aerodynamic Rotor Performance Optimization Studies . . . . . . . 37

2.4.3 Optimizer Selection $\ldots \ldots \ldots \ldots \ldots \ldots \ldots \ldots \ldots \ldots .41$

3 The Qoptr Simulation Modules $\quad 42$

3.1 Generating Two-Dimensional Airfoil Properties . . . . . . . . . . 43

3.1.1 Lift Stall Correction for the VII Algorithm . . . . . . . . . . 43

3.1.2 Validation of Two-Dimensional Airfoil Properties . . . . . . . . 52

3.1.3 Compressibility Corrections for Two-dimensional Airfoil Data . . 55

3.2 Blade Element Momentum Theory Implementation for Hover and Axial Climb.............................. 57

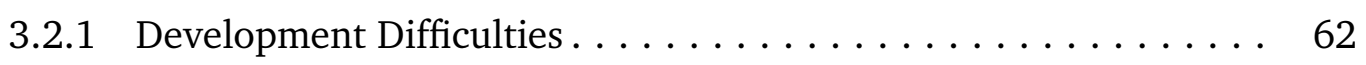

3.2.2 Validation of the Qoptr Hover Module . . . . . . . . . . . 63

3.3 Blade Element Theory Implementation for Forward Flight . . . . . . . . 67

3.3.1 Validation of the Qoptr Forward Flight Module . . . . . . . . . 79

4 Rotor Blade Optimization $\quad 85$

4.1 Optimizer Integration Verification $\ldots \ldots \ldots \ldots \ldots \ldots \ldots \ldots \ldots$

4.1.1 The Ideal Twist Rotor $\ldots \ldots \ldots \ldots \ldots \ldots \ldots \ldots \ldots \ldots$ 
4.1.2 The "Optimum Hovering Rotor" . . . . . . . . . . . . . . 87

4.1 .3 Optimizer Limitations . . . . . . . . . . . . . . . . . . 90

4.2 Optimization of a 700 Class R/C Rotor for Hover . . . . . . . . . . . 91

4.2.1 Model Rotor and Vehicle Description . . . . . . . . . . . . . . 91

4.2.2 Optimization Constants, Variables and Constraints . . . . . . . . . 92

4.2 .3 Selection of Airfoils . . . . . . . . . . . . . . . 94

4.2 .4 Hover Optimization Results . . . . . . . . . . . . . . 97

5 Conclusions and Recommendations 103

5.1 Conclusions and Accomplishments . . . . . . . . . . . . . . 103

5.2 Recommended Future Extensions for the Qoptr Framework . . . . . . . 104

$\begin{array}{ll}\text { List of References } & 106\end{array}$

$\begin{array}{lll}\text { Appendix A Airfoil Characteristics Prediction Validation } & 115\end{array}$

Appendix B Additional Hover Module Validation Figures and Discussion 126

Appendix C Blade Dynamics - Equations of Motion 135

Appendix D Additional Forward Flight Module Validation Figures and Discussion 155

Appendix E Selected Rotorcraft Airfoils Evaluated at Reynolds Numbers Between 100,000 and $1,000,000 \quad 160$ 


\section{List of Tables}

2.1 Linear induced inflow model coefficients . . . . . . . . . . . . . . . 22

3.1 Lift stall estimation correlation parameters. . . . . . . . . . . 46

4.1 Simulated analytical and optimized results for the optimum hovering rotor design. ........................ 90

4.2 Baseline rotor definition, loading and atmospheric operating conditions. 92

4.3 Performance parameters for the baseline and optimized rotor at the de-

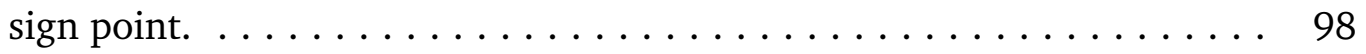

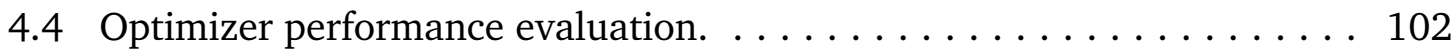

B.1 Test condition and rotor characteristics: variation of taper ratio. . . . . 128

B.2 Test condition and rotor characteristics: advanced airfoil selection. . . . 130

B.3 Test condition and rotor characteristics: variation of spanwise taper ini-

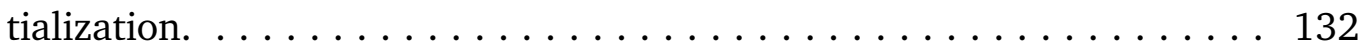

B.4 Test condition and rotor characteristics: full scale results. . . . . . . . . . 134

D.1 Scaled $\mathrm{CH}-47 \mathrm{C}$ rotor characteristics and test condition. . . . . . . . . 156

E.1 Selected rotorcraft airfoil parameters at $\mathrm{Re}=100,000 \ldots \ldots \ldots \ldots \ldots 1$

E.2 Selected rotorcraft airfoil parameters at $\mathrm{Re}=200,000 \ldots \ldots \ldots \ldots$. . . 162

E.3 Selected rotorcraft airfoil parameters at $\mathrm{Re}=500,000 \ldots \ldots \ldots \ldots$

E.4 Selected rotorcraft airfoil parameters at $\mathrm{Re}=1,000,000 . \ldots \ldots \ldots$. . . 164 


\section{List of Figures}

1.1 The Responder UAS marketed by ING Robotic Aviation. . . . . . . . . . 3

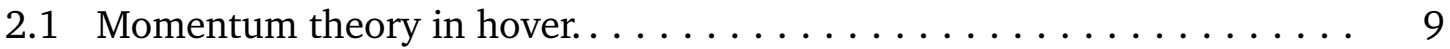

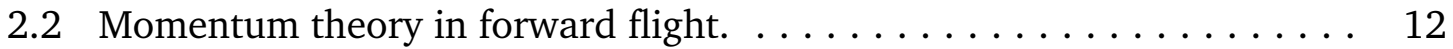

2.3 Velocity and force components at a blade section. . . . . . . . . . . 15

2.4 Blade flapping and coning induced velocities. . . . . . . . . . . . . 21

3.1 Histograms of error in the estimated lift coefficient maximum point coordinates on the lift polar. . . . . . . . . . . . . 48

3.2 Great Planes PT40 airfoil lift coefficient polars showing the VII output ( $m=0)$ and the empirical stall estimate at $R e=400,000 \ldots \ldots \ldots 0$

3.3 Eppler E472 airfoil lift coefficient polars showing the VII output $(m=0)$ and the empirical stall estimate at $R e=100,000 \& 500,000 \ldots \ldots \ldots 1$

3.4 Power coefficient and figure of merit simulated using the hover module compared to experimental results. . . . . . . . . . . . .

3.5 Error in simulated power coefficient and figure of merit predicted using the hover module. . . . . . . . . . . . . . . . . . . . . 64

3.6 Comparison of simulated power coefficient and figure of merit using XFOIL and experimentally sourced NACA 0012 airfoil data with respect to experimental results. . . . . . . . . . . . . . . . . . . . . 66 
3.7 Error in simulated power coefficient and figure of merit predicted using XFOIL and experimentally sourced NACA 0012 airfoil data. . . . . . . . 67

3.8 Fully articulated rotor hub layout in the hub-based rotating reference frame. ............................... 69

3.9 Soft in-plane teetering rotor hub layout in the hub-based rotating refer-

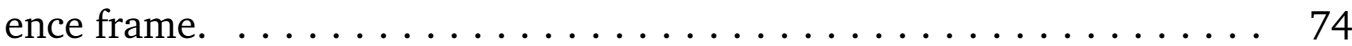

3.10 Comparison of forward flight module simulated results to experimental

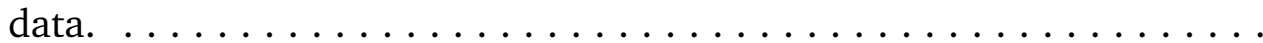

3.11 Comparison of forward flight module simulated results to experimental data (assuming teetering rotor hub) . . . . . . . . . . 83

4.1 Optimization of twist distribution for a rotor with a rectangular planform. 87

4.2 Optimization of twist and chord distributions in hover. . . . . . . . . . 89

4.3 Maximum lift coefficient of rotorcraft airfoils at Reynolds numbers between 100,000 and $1,000,000 . \ldots \ldots \ldots \ldots \ldots \ldots$

4.4 Maximum lift to drag ratio of rotorcraft airfoils at Reynolds numbers between 100,000 and $1,000,000 \ldots \ldots \ldots \ldots \ldots$

4.5 Radial performance parameter variations at the design point. . . . . . . 99

4.6 Rotor performance at off-design conditions. . . . . . . . . . . . . . . 101

A.1 Comparison of two-dimensional airfoil property data from VII including the stall estimation model and XFOIL with respect to experimental data from the University of Illinois at Urbana-Champaign. . . . . . . . . . . . . 116

A.2 Comparison of two-dimensional airfoil property data from VII including the stall estimation model and XFOIL with respect to experimental data from Universität Stuttgart. . . . . . . . . . . . . . . . . . . . . . 121 
B.1 Power coefficient and figure of merit simulated using the hover module compared to experimental results for rotors with varying taper ratios. . . 127

B.2 Power coefficient and figure of merit simulated using the hover module compared to experimental results for rotors with different airfoils. . . . 129

B.3 Power coefficient and figure of merit simulated using the hover module compared to experimental results for rotors with different spanwise taper

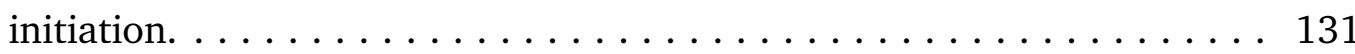

B.4 Power coefficient and figure of merit simulated using the hover module compared to full scale experimental results. . . . . . . . . . . . . . 133

C.1 Fully articulated rotor hub layout in hub-based rotating reference frame. 136

C.2 Teetering rotor hub layout in hub-based rotating reference frame. . . . . 146

D.1 Comparison of forward flight module simulated results to experimental data: collective sweep. . . . . . . . . . . . . . . . . . . . 157

D.2 Comparison of forward flight module simulated results to experimental data: shaft angle of attack sweep. . . . . . . . . . . . . . . . . 159 


\section{Nomenclature}

This thesis uses S.I. units unless otherwise specified.

$\begin{array}{ll}a & \text { Speed of sound } \\ A & \text { Rotor disk area } \\ A_{b} & \text { Total blade planform area } \\ a_{c} & \text { Lift stall correction coefficient } \\ b & \text { Airfoil semi-chord } \\ \text { BEMT } & \text { Blade element momentum theory } \\ \text { BET } & \text { Blade element theory } \\ c & \text { Chord length } \\ c_{0} & \text { Mangler and Squire Fourier series coefficient } \\ C_{a r b} & \text { Arbitrary constant } \\ C_{D} & \text { Blade element drag coefficient } \\ C_{F_{x}}, C_{F_{r}}, C_{F_{z}} & \text { Aerodynamic force coefficients in the rotating reference frame } \\ C_{H} & \text { Rotor drag force coefficient } \\ C_{l}, C_{d}, C_{m} & \text { 2D airfoil lift, drag and pitching moment coefficients } \\ C_{L} & \text { Blade element lift coefficient } \\ C_{l_{\max }} & \text { Maximum 2D airfoil lift coefficient } \\ C_{l_{x}} & \text { Lift stall correction model transition lift coefficient } \\ C_{l_{a}} & \end{array}$




\begin{tabular}{|c|c|}
\hline$C_{M_{x}}$ & Rotor rolling moment coefficient \\
\hline$C_{M_{y}}$ & Rotor pitching moment coefficient \\
\hline$c_{n}$ & Mangler and Squire Fourier series coefficient \\
\hline$C_{p}$ & Pressure coefficient at airfoil surface \\
\hline$C_{P}$ & Total power coefficient \\
\hline$C_{P_{i}}$ & Induced power coefficient \\
\hline$C_{p_{\text {inc }}}$ & Incompressible pressure coefficient \\
\hline$C_{P_{o}}$ & Profile power coefficient \\
\hline$C_{Q}$ & Rotor torque coefficient \\
\hline$C_{T}$ & Thrust coefficient \\
\hline$C_{Y}$ & Rotor side force coefficient \\
\hline$C_{\zeta}$ & Lead-lag damping coefficient \\
\hline $\mathrm{cam} / \mathrm{c}$ & Relative airfoil camber \\
\hline CFD & Computational fluid dynamics \\
\hline COTS & Commercial off the shelf \\
\hline$D$ & Drag force at blade section \\
\hline $\mathrm{d} C_{T_{a v e}}(r)$ & Mean radial thrust coefficient distribution \\
\hline $\mathrm{d} m$ & Blade mass element \\
\hline$e_{f l}$ & Non-dim. flapping hinge location \\
\hline$e_{l l}$ & Non-dim. lead-lag hinge location \\
\hline$E_{k}$ & Kinetic energy \\
\hline$E_{p}$ & Potential energy \\
\hline$e_{u s}$ & Non-dim. undersling distance \\
\hline$f$ & Optimization objective function \\
\hline$F$ & Prandtl's blade loading correction \\
\hline$F_{\text {root }}, F_{\text {tip }}$ & Prandtl's loading correction at the blade root and tip \\
\hline
\end{tabular}




$\begin{array}{ll}F_{x}, F_{r}, F_{z} & \text { Aerodynamic force components in the rotating reference frame } \\ F M & \text { Figure of merit } \\ \vec{g} & \text { Gravitational acceleration } \\ H & \text { Rotor drag force } \\ \hat{i}, \hat{j}, \hat{k} & \text { Principle direction unit vectors in the rotating reference frame } \\ I_{C o r} & \text { Equation of motion Coriolis effects } \\ I_{\beta} & \text { Blade moment of inertia about the flapping hinge } \\ I_{\beta_{T}} & \text { Rotor moment of inertia about the teetering hinge } \\ I_{\zeta} & \text { Blade moment of inertia about the lead-lag hinge } \\ \text { ISA } & \text { International standard atmosphere model } \\ j & \text { Iteration number } \\ K_{\beta} & \text { Flapping hinge spring stiffness } \\ k_{x} & \text { Longitudinal linear induced inflow model coefficient } \\ k_{y} & \text { Lateral linear induced inflow model coefficient } \\ k & \text { Reduced frequency } \\ L & \text { Lift force at blade section } \\ m & \text { VII empirical free-shear layer angle coefficient } \\ \dot{m} & \text { Mass flux } \\ M & \text { Mach number } \\ M_{\infty} & \text { Free-stream Mach number } \\ m_{d} & \text { Spanwise linear blade density } \\ M_{r} & \text { Pitching moment at blade section } \\ \left.M_{r}\right|_{\text {Blade Root }} & \text { Total aerodynamic blade pitching moment at the blade root } \\ M_{\text {Tip }} & \text { Blade tip Mach number } \\ M_{y} & \text { Rotor rolling moment } \\ & \text { Rotor pitching moment } \\ & \end{array}$




$\begin{array}{ll}\text { MAV } & \text { Micro aerial vehicles } \\ n & \text { Number of design variables (optimization) } \\ N_{b} & \text { Number of rotor blades } \\ n_{c r i t} & \text { XFOIL transition parameter } \\ \Delta p & \text { Pressure difference created across the disk } \\ P & \text { Total rotor power } \\ \Delta p_{1}, \Delta p_{3} & \text { Type I \& III Mangler and Squire inflow disk pressure distributions } \\ P_{1}, P_{2} & \text { Penalty functions } \\ \vec{P}_{\mathrm{d} m} & \text { Mass element position vector } \\ P_{i} & \text { Induced power } \\ P_{o} & \text { Profile power } \\ Q & \text { Torque } \\ q_{i} & \text { Generalized degree of freedom } \\ Q_{q_{i}} & \text { Non-conservative work } \\ Q_{\beta} & \text { Non-conservative work for the flap degree of freedom } \\ Q_{\zeta} & \text { Non-conservative work for the lag degree of freedom } \\ r & \text { Non-dim. radial blade station } \\ R & \text { Rotor radius } \\ r_{0} & \text { Non-dim. rotor root cut-out } \\ R_{\text {Tot }} & \text { Resultant force at the blade section } \\ \text { RANS } & \text { Reynolds averaged Navier Stokes equations } \\ \text { r/c } & \text { Radio-controlled } \\ R e & \text { Reynolds number } \\ \text { ref } & \text { Vehicle fixed hub-based reference frame } \\ s_{e r r} & \text { Standard error } \\ T & \text { Rotor thrust } \\ & \end{array}$




$\begin{array}{ll}t / c & \text { Relative airfoil thickness } \\ \text { TPP } & \text { Tip path plane } \\ U & \text { Resultant velocity at the blade section } \\ U_{n d} & \text { Non-dim. Resultant velocity at the blade section } \\ U_{P} & \text { Perpendicular blade section velocity component } \\ U_{R} & \text { Radial blade section velocity component } \\ U_{T} & \text { Tangential blade section velocity component } \\ \text { UAS } & \text { Unmanned aerial system } \\ \text { UAV } & \text { Unmanned aerial vehicle } \\ V_{\infty} & \text { Total rotor airspeed } \\ V_{a v e} & \text { Mean velocity } \\ V_{c} & \text { Climb velocity } \\ \vec{V}_{\mathrm{d} m} & \text { Mass element velocity vector } \\ v_{h} & \text { Hover induced velocity at the rotor disk } \\ v_{i} & \text { Induced velocity at the rotor disk } \\ w & \text { Slipstream exit velocity } \\ w_{1}, w_{3} & \text { Type I \& III Mangler and Squire inflow weighting factors } \\ x_{\text {cam }} & \text { Chordwise location of maximum airfoil camber } \\ x_{r e f}, y_{r e f}, z_{r e f} & \text { Principle directions in the vehicle fixed hub reference frame } \\ x_{t} & \text { Chordwise location of maximum airfoil thickness } \\ x_{T P P}, y_{T P P}, z_{T P P} & \text { Principle directions in the tip path plane coordinate system } \\ x_{y_{\max }} & \text { Chordwise location of maximum airfoil upper surface deflection } \\ Y & \text { Rotor side force } \\ y_{\max } / c & \text { Relative airfoil maximum upper surface deflection } \\ \alpha & \text { Effective blade section angle of attack } \\ \alpha_{1} & \text { Angle of attack for maximum airfoil lift to drag ratio } \\ & \end{array}$




\begin{tabular}{|c|c|}
\hline$\alpha_{o}$ & Zero lift angle of attack \\
\hline$\alpha_{\text {shaft }}$ & Shaft angle of attack \\
\hline$\alpha_{T P P}$ & Tip path plane angle of attack \\
\hline$\alpha_{x}$ & Lift stall model transition angle of attack \\
\hline$\left.\alpha\right|_{C_{l_{\max }}}$ & Angle of attack at maximum airfoil lift coefficient \\
\hline$\beta$ & Flapping hinge deflection \\
\hline$\dot{\beta}$ & Blade flapping velocity \\
\hline$\ddot{\beta}$ & Blade flapping acceleration \\
\hline$\beta^{*}$ & Non-dim. flapping velocity \\
\hline$\beta^{* *}$ & Non-dim. flapping acceleration \\
\hline$\beta_{0}$ & Coning angle \\
\hline$\beta_{\text {lat }}$ & Lateral disk tilt due to flapping \\
\hline$\beta_{\text {long }}$ & Longitudinal disk tilt due to flapping \\
\hline$\beta_{p}$ & Precone angle \\
\hline$\beta_{T}$ & Teetering hinge deflection \\
\hline$\dot{\beta}_{T}$ & Teetering velocity \\
\hline$\ddot{\beta}_{T}$ & Teetering acceleration \\
\hline$\gamma$ & Ratio of specific heats \\
\hline$\gamma_{1}, \gamma_{2}$ & Penalty function weighting coefficients \\
\hline$\zeta$ & Lead-lag hinge deflection \\
\hline$\dot{\zeta}$ & Blade lead-lag velocity \\
\hline$\ddot{\zeta}$ & Blade lead-lag acceleration \\
\hline$\zeta^{*}$ & Non-dim. lead-lag velocity \\
\hline$\zeta^{* *}$ & Non-dim. lead-lag acceleration \\
\hline$\theta$ & Blade element pitch angle \\
\hline$\theta_{75}$ & Collective pitch angle \\
\hline
\end{tabular}




\begin{tabular}{ll}
$\theta_{t i p}$ & Blade tip pitch angle \\
$\lambda$ & Total inflow ratio \\
$\lambda_{c}$ & Inflow ratio due to climb velocity \\
$\lambda_{h}$ & Hover induced inflow ratio \\
$\lambda_{i}$ & Induced inflow velocity ratio \\
$\mu$ & Total advance ratio \\
$\mu_{d}$ & Dynamic viscosity of air \\
$\mu_{K}$ & Kaplan's rule Mach number effect \\
$\mu_{x}$ & Advance ratio parallel to the tip path plane, a.k.a. tip-speed ratio \\
$\mu_{x_{r e f}}$ & Advance ratio parallel to the hub reference plane \\
$\mu_{z}$ & Advance ratio perpendicular to the tip path plane \\
$\mu_{z_{r e f}}$ & Advance ratio perpendicular to the hub reference plane \\
$v_{\beta}, v_{\beta_{T}}, v_{\zeta}$ & Non-dim. natural flapping, teetering \& lead-lag frequencies \\
$\rho$ & Air density \\
$\sigma$ & Rotor solidity ratio \\
$\sigma_{e}$ & Equivalent rotor solidity ratio \\
$\phi$ & Inflow angle \\
$\chi$ & Wake skew angle \\
$\psi$ & Azimuth angle \\
$\vec{\omega}$ & Angular velocity of rotating reference frame \\
$\Omega$ & Rotor rotational speed \\
$\omega_{C_{F_{z}}}$ & Under-relaxation factor (hover module) \\
$\omega_{k}$ & Excitation frequency \\
\hline
\end{tabular}




\section{Chapter 1}

\section{Introduction}

\subsection{Small Rotorcraft UAS Operation}

Unmanned aerial systems (UAS) - like other robotic devices - are developed to perform missions that are too "dull, dirty or dangerous" for manned vehicles. As more applications are identified the UAS market is expanding quickly. In 2013, market analysis firm Teal Group Corporation predicted unmanned aerial vehicle (UAV) spending to double from this decade to the next with global expenditures rising to USD 11.6 billion annually and totalling approximately USD 89 billion over the next ten years [1]. While UAVs and UASs are most famous for their employment in military activities for surveillance, reconnaissance and as integrated weapon systems, the number of civil applications is also growing fast. Surveillance and search and rescue functions are used by police and emergency services, commercial applications include aerial surveys, infrastructure inspection and monitoring, for example in the oil and gas sector. Conservation agencies and condo developers may also make use of aerial photography for wildlife and habitat monitoring or to generate panoramic view predictions, respectively [2].

As in the manned aviation industry, UAS also come in a variety of configurations. Rotorcraft UAS range from micro aerial vehicles (MAV) measuring only a few centimeters 
in length to large vehicles with rotor diameters on the order of 3 to 11 meters such as the Schiebel Camcopter and the Boeing A160 Hummingbird, which were developed as unmanned systems from their inception [3], [4], or the Northrop Grumman Fire Scout MQ-8B, which is based on a Schweizer 333 airframe [5]. Full size manned helicopters have also been retrofitted for autonomous operation; examples include the Northrop Grumman Fire Scout MQ-8C, which is build on a commercial Bell 407 airframe [6], the Kaman and Lockheed-Martin K-MAX UAS [7] and Airbus Helicopter's unmanned EC145, which performed demonstration flights for the company's optionally piloted vehicle program [8].

At the lower end of this spectrum hobbyists have and are continuing to develop radio-controlled $(\mathrm{r} / \mathrm{c}$ ) vehicles utilizing an increasing amount of autonomy. Commercially available $\mathrm{r} / \mathrm{c}$ rotorcraft come in many different configurations from the traditional single main rotor with tail rotor arrangement over counter-rotating rotors to multi-rotor designs and full vehicle costs ranging from approximately \$ 100 to \$ 4500 depending on size and manufacturer [9]-[11]. With advances in autonomous flight as well as miniaturization of sensors and real-time communication systems, numerous companies are starting to offer rotorcraft UAS services using vehicles which are often based on $\mathrm{r} / \mathrm{c}$ helicopters or otherwise rely on $\mathrm{r} / \mathrm{c}$ components for aeromechanical systems. The size and relatively low cost of these small rotorcraft UAS makes them ideal for urban implementation in support of paramilitary and emergency operations and economically viable to smaller commercial endeavours. One such example is the ING Robotic Aviation Responder UAS pictured in Figure 1.1.

Unfortunately, the flight requirements of $\mathrm{r} / \mathrm{c}$ helicopters and small rotorcraft UAS have little in common. $\mathrm{R} / \mathrm{c}$ helicopters depend on high control authority and thrust margins for agile aerobatic flight manoeuvres. UAS requirements, on the other hand, 


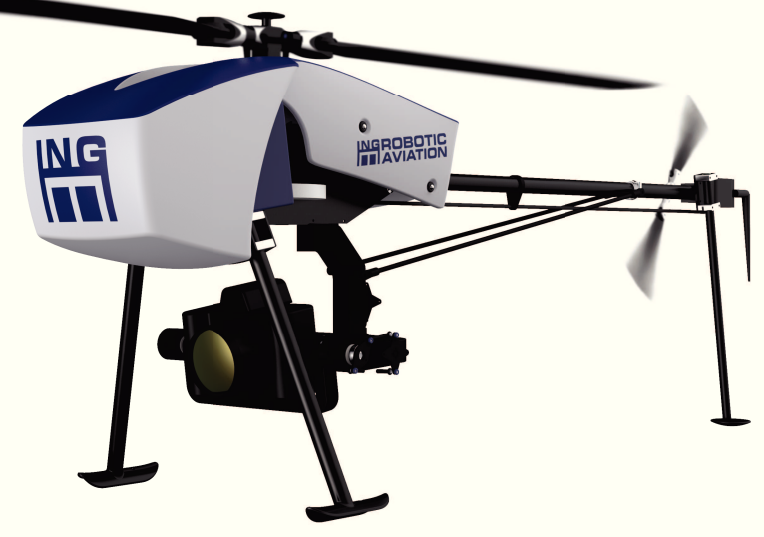

Figure 1.1: The Responder UAS marketed by ING Robotic Aviation [12].

include efficient hover, long endurance and significant payload mass fractions, which align more closely with design drivers of full-sized helicopters.

One of the major disadvantages of $r / c$ helicopters compared to their full-size counterparts is that $\mathrm{r} / \mathrm{c}$ helicopter main rotors are significantly less efficient. A common nondimensional parameter used to express helicopter hover efficiency is the rotor's figure of merit (FM), which is an expression for the ratio of ideal power required, based on simple momentum theory, and the actual power required to hover [13, pp. 70-71]. In a Carleton University undergraduate wind tunnel experiment the figure of merit for an $\mathrm{r} / \mathrm{c}$ helicopter ${ }^{1}$ was experimentally determined to be approximately $25 \%$, while modern full-sized helicopters achieve figures of merit on the order of 70 to $80 \%$ [13, p. 280].

The main reason for this large discrepancy is the fact that $\mathrm{r} / \mathrm{c}$ helicopters are built for 3D aerobatic flight, where the vehicles are expected to have almost instantaneous acceleration for metronome or tic-toc manoeuvres and the flight regime also includes

\footnotetext{
${ }^{1}$ The Walkera model V400D02 helicopter has a rotor diameter of $0.64 \mathrm{~m}$ and an approximate take-off mass of $0.5 \mathrm{~kg}$.
} 
extended periods of inverted flight and hover [14]. These requirements clearly benefit from large thrust margins as well as symmetric main rotor thrust reversal, which lead to simple untwisted rotor blade designs featuring symmetric airfoils ${ }^{2}$.

Another source for the inefficiency of $\mathrm{r} / \mathrm{c}$ rotors is the Reynolds number range in which the rotor blades operate. Depending on the size of the UAS, typical chord-based Reynolds numbers are approximately three orders of magnitude smaller than those seen on full-sized rotors [13, p. 412]. As Reynolds number decreases the performance characteristics of airfoils decline [16]. While some references for low Reynolds number airfoils exist [17]-[19], little data is available for rotorcraft specific airfoil development in this Reynolds number regime.

Lastly, economic factors likely limit the amount of engineering or scientific development of $\mathrm{r} / \mathrm{c}$ rotor blades as even a high end blade pair in the 700 class $^{3}$ is generally available at prices ranging from USD 100 to USD 200 [9], [10], [20]. This is supported by the fact that blades are usually fitted with a single airfoil along the whole blade span and other features like special tip shapes are rarely implemented on $\mathrm{r} / \mathrm{c}$ rotor blades.

\subsection{Objective}

The objective of this work is the development of a rotor blade performance simulation and optimization framework specifically suited to typical UAS operating conditions and the challenges of the low Reynolds number environment encountered by large $\mathrm{r} / \mathrm{c}$ helicopters. In order to allow continued use of commercial off the shelf (COTS) components

\footnotetext{
${ }^{2}$ A notable exception are a number of rotor blade models offered by SpinBlades, which take advantage of cambered airfoils [15].

${ }^{3} \mathrm{R} / \mathrm{c}$ helicopters in the 700 class use main rotor blades measuring approximately $700 \mathrm{~mm}$ in length. Including the dimensions of the hub, this results in an approximate rotor diameter of 1.5 to $1.6 \mathrm{~m}$.
} 
the rotor blades are to be completely compatible with typical 700 class $r / c$ hub systems ${ }^{4}$. The rotor configuration is limited to conventional single main and tail rotors. Numerical operations are to be carried out at small computational cost to produce a fast rotor simulation environment for the Carleton University Rotorcraft Research Group and a practical design tool for a rotorcraft UAS engineer.

\subsection{Organization of Thesis}

This thesis documents the development of the Qoptr simulation and optimization framework. Chapter 2 combines the literature review with a summary of published methods and theories that various portions of the framework are based on.

Chapter 3 outlines the main tasks completed in the development of the simulation framework. Two pieces of software are evaluated on their capability to generate reliable low Reynolds number airfoil performance data. Then the theoretical procedures within the hover and forward flight simulation modules of the Qoptr framework are described in detail and validated.

The integration of the hover module with a MATLAB optimizer is demonstrated in Chapter 4. Chapter 5 concludes the thesis and lists a number of further developments that may be implemented in the future in order to improve the simulation results and extend the capabilities of the Qoptr framework.

A number of appendices contain additional comparisons to published experimental data, the derivation of the equations of motion for the rotor blades in forward flight and other reference material. Cross-references are provided in the respective section of the text.

\footnotetext{
${ }^{4}$ See note 3
} 


\section{Chapter 2}

\section{Literature Review and Theory Background}

The following sections outline research efforts made in studying scaled rotors, describe some of the methods used to predict the aerodynamic performance capabilities of helicopter rotors and discuss airfoil data requirements and considerations related to rotorcraft operations. A short section then summarizes optimization efforts applied to rotorcraft design. The selection of various simulation and optimization methods needed to satisfy the objective are discussed in the respective sections.

\subsection{Review of Small Rotor Performance Studies}

Scaled rotors have been used to perform experimental studies in support of full scale helicopter rotor developments. In the 1980s and early 1990s several studies were conducted at the NASA Langley Research Center using the 2-meter rotor test system [21][23]. These studies usually involved a four-bladed baseline rotor configuration and one or more test rotors of equal thrust-weighted solidity, which were tested on a hover stand with a generic helicopter fuselage shell. Reference [21] considered the effect of different taper ratios applied to the outer $20 \%$ of the blade span at different rotational speeds, Reference [22] describes the effect of advanced airfoils compared to a rotor with NACA 0012 airfoils and Reference [23] examined the effect of the radial 
position of taper initiation. Each reference provided experimental thrust and power coefficients as well as figure of merit data. Maximum figure of merit values between 0.6 and 0.75 were recorded for the various configurations usually at thrust coefficients between 0.006 and 0.008 . Tip Reynolds numbers were in the range of approximately $1.6 \times 10^{5}$ to $3.8 \times 10^{51}$.

Reference [24] outlines the efforts made to improve the hover efficiency of a $1.2 \mathrm{~m}$ diameter electric $\mathrm{r} / \mathrm{c}$ helicopter, performed at the University of Maryland in response to the 2010 American Helicopter Society student design competition. A number of experimental and analytical tests were performed to investigate the effect of rotor speed, blade twist and taper. A custom designed blade, which also incorporated a low-drag airfoil, resulted in a rotor thrust increase of $17 \%$ at the design power level of $0.6 \mathrm{hp}$ compared to the baseline set of COTS Mylar covered wooden blades. However another set of carbon fibre "high performance" COTS blades outperformed the baseline by 25\% at the same power level. Approximate tip Reynolds numbers for the experimental tests were in the range of $2.75 \times 10^{5}$ to $3.4 \times 10^{5}$. No experimental figure of merit values were stated, but the theoretical design point was set at a value of 0.66 .

In Reference [25] another experimental investigation of rotor blades with diameters ranging from $1.26 \mathrm{~m}$ to $1.62 \mathrm{~m}$ was conducted to determine their performance capability for a coaxial UAV implementation. The rotor blades had no twist or taper with a NACA 0015 section. They were tested in a single rotor configuration test rig. Maximum figure of merit values of 0.59 to 0.65 were recorded at blade loading coefficients ${ }_{T} / \sigma$ between 0.09 and 0.11 . One of the goals of the investigation was the determination of the induced power factor $\kappa$ which was shown to vary from 1.35 to 1.42 . Approximate tip Reynolds number ranged from $2.8 \times 10^{5}$ to $4.3 \times 10^{5}$.

\footnotetext{
${ }^{1}$ Additional findings made in References [21], [22] and [23] with respect to test rotor characteristics are summarized in Appendix B.
} 
Since the introduction of the MAV concept by the Defense Advanced Research Project Agency (DARPA) in 1996 numerous research studies have also been conducted to investigate and improve the performance capabilities of this class of aircraft. MAV category aircraft are constrained dimensionally to $15 \mathrm{~cm}$ and by weight to about 100 grams. Because of the size limitation, rotorcraft MAV rotors operate at very low Reynolds numbers, usually significantly less than 60,000. References [26], [27], [28] and [29] report figures of merit for a variety of these rotors in the range of 0.4 to 0.62 with tip Reynolds numbers between 25,000 and 46,000. These Reynolds numbers are deemed significantly lower than the range considered in this study.

\subsection{Aerodynamic Performance Analysis Methods for Helicopter Rotors}

This section contains a short history of rotor performance methods as well as developments and equations relevant to methodologies utilized in this work.

\subsubsection{Momentum Theory}

Momentum theory dates back to 1865 when Rankine originally introduced it to aid in the analysis of marine propellers. In the early 20th century it was applied to aircraft propellers [30]. Momentum theory is a form of actuator disk theory, which considers the helicopter rotor as having an infinite number of blades, essentially forming a circular disk. This disk is assumed to be infinitely thin, but able to support a pressure difference. The flow through the rotor is assumed incompressible and constrained to a well defined slipstream. As the name suggests, the theory is based on the conservation laws of fluid 
mechanics, namely conservation of mass, momentum and energy.

\subsubsection{Momentum Theory in Hover}

In the literature, momentum theory is often divided into "basic momentum theory" and more advanced variations, which extend the theory to include more flow effects. Basic momentum theory assumes constant one-dimensional flow at each cross section of the slipstream, neglecting swirl in the wake and hence the effect of angular momentum in general. The method is used as an initial estimate of wake induced flow velocity and the induced power loss, making it the basis for the rotor's hover efficiency parameter, the figure of merit.

Applied to the hover state as shown in Figure 2.1, the flow enters the slipstream at zero velocity and exits the slipstream at a finite velocity $w$. The disk has area $A$ and produces thrust $T$. At the disk the slipstream velocity, or induced velocity, is $v_{i}$.

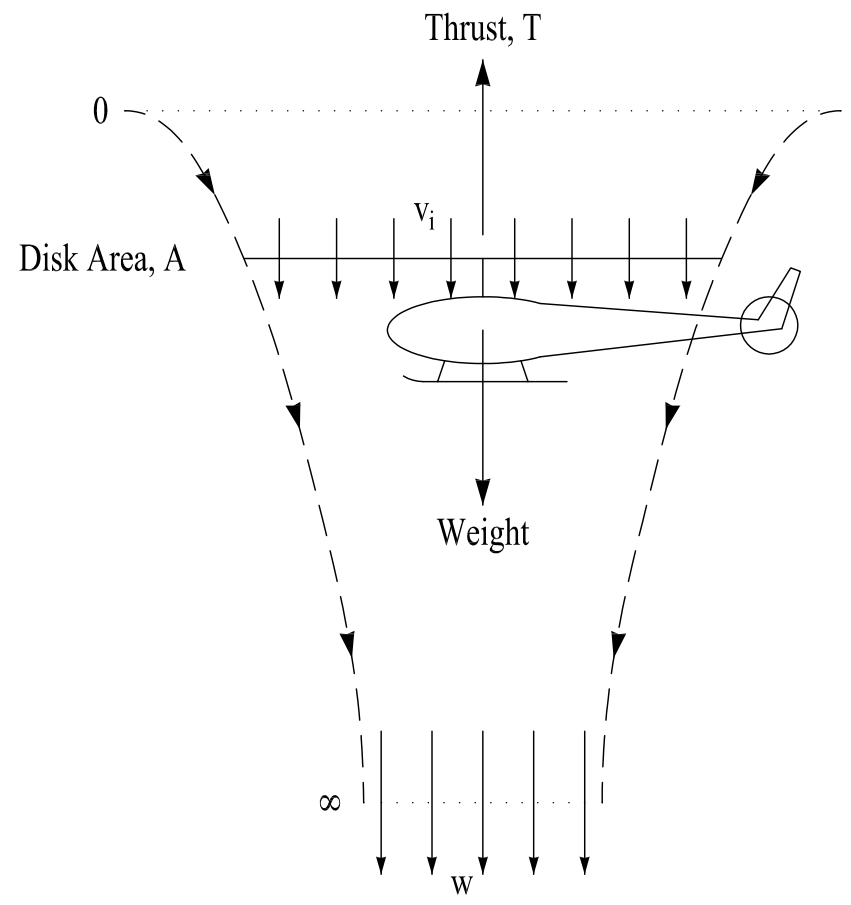

Figure 2.1: Momentum theory in hover. 
Considering the slipstream as a one-dimensional stream tube allows the following definitions for mass flux, the rate of change of momentum and the rate of change of energy.

$$
\begin{gathered}
\dot{m}=\rho A v_{i} \\
T=\dot{m} w \\
T v_{i}=\frac{1}{2} \dot{m} w^{2}
\end{gathered}
$$

Eliminating $w$ from Equations 2.2 and 2.3, expanding the mass flux term using Equation 2.1 and solving for thrust gives

$$
T=2 \rho A v_{i}^{2}
$$

Equation 2.4 can be solved for the hover induced velocity $v_{i}$, sometimes denoted by a subscript $h$.

$$
v_{h}=\sqrt{\frac{T}{2 \rho A}}
$$

The induced power loss $P_{i}$ is defined as the amount of power required to generate thrust. Equation 2.6 shows the induced power loss in hover.

$$
P_{i}=T v_{h}=T \sqrt{\frac{T}{2 \rho A}}
$$

For theoretical analyses parameters are often cast in non-dimensional form. For helicopter rotors, air density $\rho$, radius $R$ and tip speed $\Omega R$ are used for this purpose. The induced velocity ratio is represented by $\lambda_{i}$ and $C_{T}$ and $C_{P_{i}}$ designate the thrust and induced power coefficients, respectively.

$$
\lambda_{h}=\frac{v_{h}}{\Omega R}
$$




$$
\begin{gathered}
C_{T}=\frac{T}{\rho \pi R^{2}(\Omega R)^{2}}=\frac{2 \rho A v_{i}^{2}}{\rho \pi R^{2}(\Omega R)^{2}}=2 \lambda_{h}^{2} \\
C_{P_{i}}=\frac{P_{i}}{\rho \pi R^{2}(\Omega R)^{3}}=\frac{T}{\rho \pi R^{2}(\Omega R)^{2}} \frac{v_{h}}{(\Omega R)}=C_{T} \lambda_{h}
\end{gathered}
$$

Solving the thrust coefficient expression (Eq. 2.8) for $\lambda_{h}$ and substituting into Equation 2.9 gives

$$
C_{P_{i}}=\frac{C_{T}^{\frac{3}{2}}}{\sqrt{2}}
$$

It can be shown mathematically that the induced power is minimum when inflow through the disk is constant. Since this is one of the assumptions of basic momentum theory, the induced power defined here represents the optimal power requirement of an ideal rotor. The figure of merit is defined as the ratio of this ideal power $P_{i}$ required to generate thrust in hover and the actual total power of the rotor in hover $P$ as shown in Equations 2.11 and 2.12.

$$
\begin{gathered}
F M=\left.\frac{\text { ideal power }}{\text { actual total power }}\right|_{\text {in hover }} \\
F M=\frac{C_{P_{i}}}{C_{P}}=\frac{C_{T}^{\frac{3}{2}}}{\sqrt{2} C_{P}}
\end{gathered}
$$

Various extensions to the basic momentum theory in hover allow incorporation of varying inflow across the rotor disk, addition of the angular momentum equation and swirl in the wake flow. Basic momentum theory can also be applied to axial flight. Here, however, it is limited to climb and fast descend rates. At moderate descend rates, where the climb velocity $V_{c}$ is between $-2 v_{h}$ and 0 , the slipstream becomes less well defined due to partial recirculation of flow at the disk. These conditions disagree with the one-dimensional flow assumption and therefore invalidate the actuator disk model. 


\subsubsection{Momentum Theory in Forward Flight}

In 1926, Glauert also applied momentum theory to forward flight [30, p. 127]. Operating with a forward velocity requires a propulsive force to overcome drag. This force is provided by inclining the rotor disk toward the flight direction. The rotor inclination angle $\alpha_{T P P}$ is introduced to define the orientation of the "tip path plane" with respect to the incoming flow. Additionally, two advance ratios $\mu_{x}$ and $\mu_{z}$ quantify the incoming flow as non-dimensional velocity components parallel and perpendicular to the plane of the rotor, respectively. $\mu_{x}$ is also known as the tip-speed ratio. Expressions for the advance ratios and the total inflow ratio in forward flight are shown in Equations 2.13 to 2.15 [13, pp. 95-99], [31, pp. 111-115].

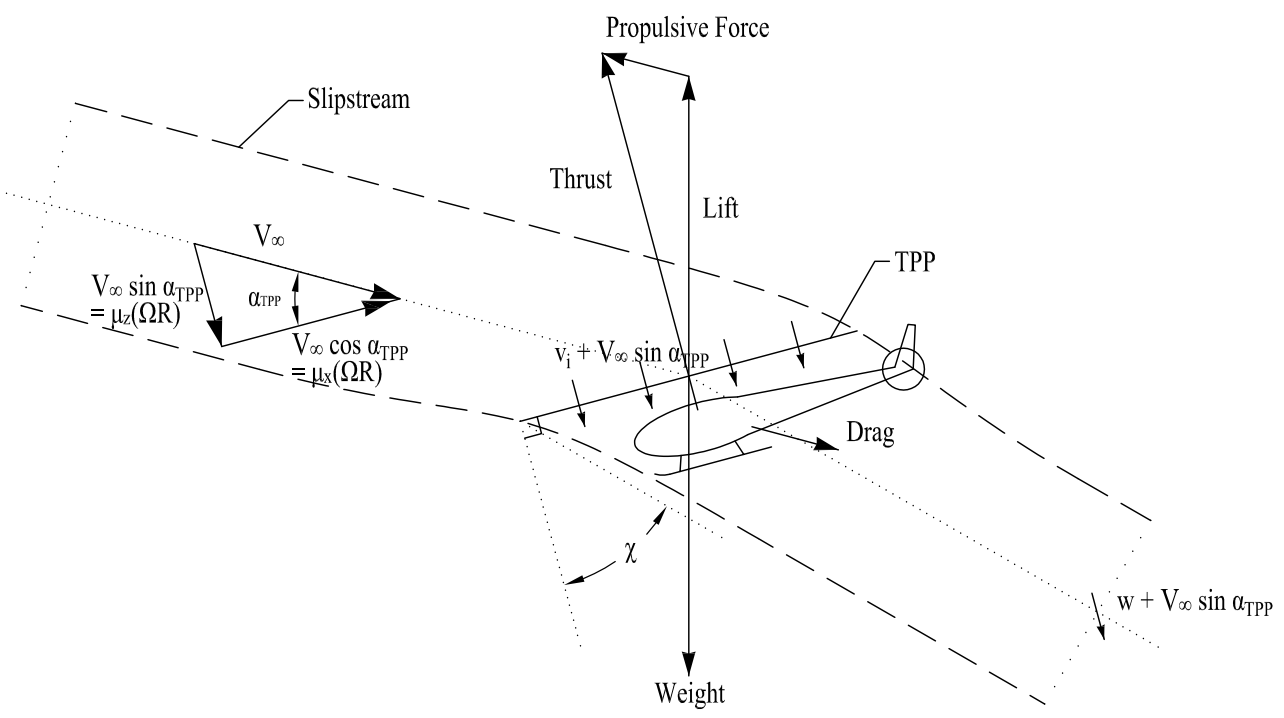

Figure 2.2: Momentum theory in forward flight.

$$
\begin{aligned}
& \mu_{x}=\frac{V_{\infty} \cos \alpha_{T P P}}{\Omega R} \\
& \mu_{z}=\frac{V_{\infty} \sin \alpha_{T P P}}{\Omega R}
\end{aligned}
$$




$$
\lambda=\mu_{z}+\lambda_{i}
$$

Glauert then related the induced inflow ratio $\lambda_{i}$ in forward flight to the induced inflow in hover $\lambda_{h}$.

$$
\lambda_{i}=\frac{\lambda_{h}^{2}}{\sqrt{\mu_{x}^{2}+\left(\mu_{z}+\lambda_{i}\right)^{2}}}=\frac{C_{T}}{2 \sqrt{\mu_{x}^{2}+\lambda^{2}}}
$$

Equations 2.13 to 2.16 also apply to combined forward flight and climb or descend. In this case $V_{\infty}$ represents the vector sum of the forward and climb velocities and $\alpha_{T P P}$ is measured with respect to this total velocity. Once again, there is a limitation on the applicability of the theory at flight conditions with an upward component of velocity normal to the rotor between 0 and $2 v_{h}$ as the one-dimensional flow assumption is not valid [13, p. 99].

\subsubsection{Alternative Actuator Disk Theories}

As mentioned previously, momentum theory is a form of actuator disk theory. Alternative approaches to defining the flow for the actuator disk model have also been developed based on vortex theory and potential flow theory [30, p. 29]. Johnson provides a good summary of the development of vortex theory and its application to helicopter rotors [30, pp. 72-91]. Vortex theory was developed to analyse propeller aerodynamics with initial contributions by Betz and Glauert around 1920. It allows a more realistic analysis of the rotor wake, which contains strong tip vortices due to large gradients in blade loading at the blade tip. To take account of the effect of discrete propeller blades - in contrast with the "infinite number of blades" assumption of the basic actuator disk model - Prandtl approximated the vorticity in the far wake using a two-dimensional model in 1919 [32] and derived a tip loading correction factor F published by Glauert in 1935 [33, pp. 261-269]. Goldstein went a step further to find the exact solution for 
a helical wake moving at constant axial velocity in 1929. Both developments are most accurate for high inflow propellers. However, helicopter rotors usually have low inflow velocities causing the tip vortices of previous blades and rotations to affect the flow at the rotor. While these interactive effects are not captured by the Prandtl or Goldstein model, Johnson [30, p. 88] states that the Prandtl implementation is justified by its simplicity when the rotor wake is not further analysed in detail. Prandtl's blade loading correction is shown in Equation 2.17 [30, p. 85].

$$
F=\frac{2}{\pi} \cos ^{-1}\left(e^{-\frac{N_{b}(1-r)}{2 r \phi}}\right)
$$

\subsubsection{Blade Element Theory}

Almost all helicopter aerodynamic analyses are based on blade element theory (BET). Stefan Drzewiecki is credited with the major introduction of blade element theory between 1892 and 1920, though contributions were also made by Froude and Lanchester [30, pp. 46-47], [13, p. 115]. Blade element theory is lifting-line theory applied to a rotating wing. In lifting-line theory the wing has a high aspect ratio and the aerodynamics are solved in two parts. The wing is divided into two-dimensional sections and at each section the loading is calculated. The effect of the wake is accounted for using an induced downwash velocity component. The second portion of the theory models the bound circulation at the wing as a vortex line that sheds vorticity into the wake. The shed vorticity then determines the induced downwash velocity. Thus the two portions of the theory interact by passing blade loading from the first to the second and induced velocity from the second to the first part. Spanwise discretization of the blade allows arbitrary variations of blade parameters along the span and the analysis of the resulting effects on the performance of the rotor. Blade element analysis assumes that 
adjacent elements have no influence on each other and that three-dimensional effects are negligible or may be captured through empirical factors.

The flow field at an individual blade section is shown schematically in Figure 2.3. Velocities at the section can be separated into three orthogonal components where $U_{T}$ is the tangential velocity, $U_{P}$ is the perpendicular component and $U_{R}$ is the radial velocity. Generally, the influence of $U_{R}$ on the lift of the blade section is ignored according to the principle that a swept wing section generates lift due to the velocity component perpendicular to the section. Equations 2.18 and 2.19 are expressions for velocities $U_{P}$ and $U_{T}$ in hover and axial flight in terms of the non-dimensional radial position $r$.

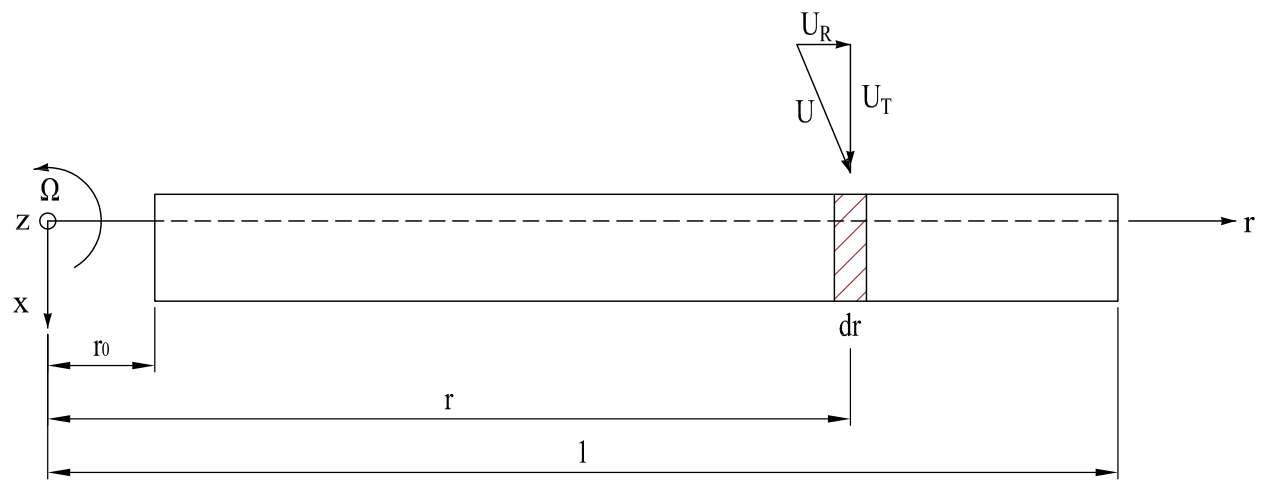

(a) Top view.

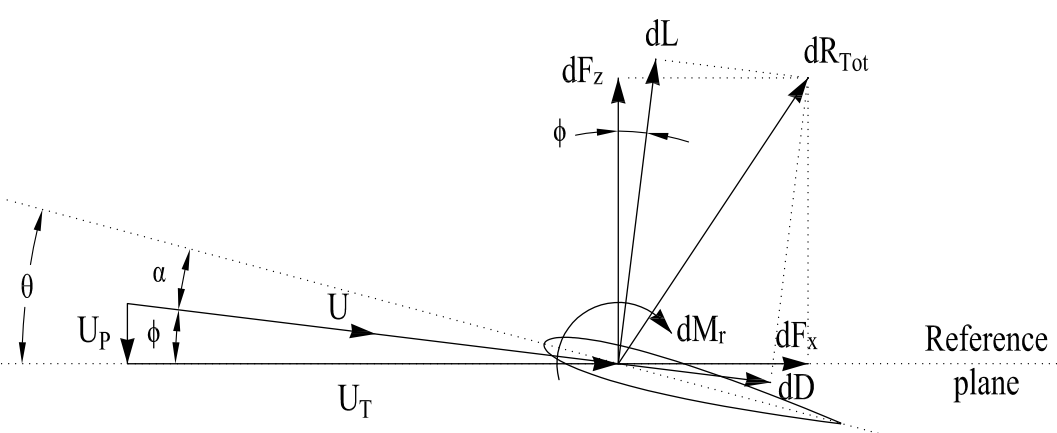

(b) Blade element view.

Figure 2.3: Velocity and force components at a blade section. 


$$
\begin{gathered}
U_{P}=V_{c}+v_{i} \\
U_{T}=\Omega r R
\end{gathered}
$$

From these components the resultant velocity at the blade section $U$ and the inflow angle $\phi$ can be obtained.

$$
\begin{gathered}
U=\sqrt{U_{T}^{2}+U_{P}^{2}} \\
\tan \phi=\frac{U_{P}}{U_{T}}
\end{gathered}
$$

Therefore a blade element operating at a pitch angle $\theta$ relative to the reference plane will have an effective angle of attack $\alpha$ defined in Equation 2.22.

$$
\alpha=\theta-\phi
$$

Blade angle lift and drag coefficients represent forces relative to the resultant velocity at the section and must therefore be decomposed into values relative to the reference plane before they are integrated along the blade. As shown in Figure 2.3 this requires a coordinate transformation through inflow angle $\phi$.

$$
\begin{aligned}
& \mathrm{d} F_{z}=\mathrm{d} L \cos \phi-\mathrm{d} D \sin \phi \\
& \mathrm{d} F_{x}=\mathrm{d} L \sin \phi+\mathrm{d} D \cos \phi
\end{aligned}
$$

From these quantities, the contributions to thrust, torque and power are calculated as shown in Equations 2.25 to 2.27.

$$
\mathrm{d} T=N_{b} \mathrm{~d} F_{z}
$$




$$
\begin{aligned}
\mathrm{d} Q & =N_{b} \mathrm{~d} F_{x} r R \\
\mathrm{~d} P & =N_{b} \mathrm{~d} F_{x} \Omega r R
\end{aligned}
$$

Non-dimensionalizing gives the following expressions for elemental thrust, torque and power coefficients.

$$
\begin{gathered}
\mathrm{d} C_{T}=\frac{\mathrm{d} T}{\rho \pi R^{2}(\Omega R)^{2}}=\frac{\sigma}{2}\left(C_{L} \cos \phi-C_{D} \sin \phi\right) \frac{U^{2}}{(\Omega R)^{2}} \mathrm{~d} r \\
\mathrm{~d} C_{Q}=\mathrm{d} C_{P}=\frac{\mathrm{d} P}{\rho \pi R^{2}(\Omega R)^{3}}=\frac{\sigma}{2}\left(C_{L} \sin \phi+C_{D} \cos \phi\right) \frac{U^{2}}{(\Omega R)^{2}} r \mathrm{~d} r
\end{gathered}
$$

$C_{L}$ and $C_{D}$ represent the element lift and drag coefficients. The rotor solidity ratio $\sigma$ relates the blade area to the rotor disk area as shown in Equation 2.30 assuming constant chord $c$.

$$
\sigma=\frac{N_{b} A_{b}}{A}=\frac{N_{b} R c}{\pi R^{2}}=\frac{N_{b} c}{\pi R}
$$

Introducing coefficients for the forces perpendicular to and in plane with the rotor disk allows Equations 2.28 and 2.29 to be rewritten as follows.

$$
\begin{gathered}
\mathrm{d} C_{T}=\frac{\sigma}{2} C_{F_{z}} \frac{U^{2}}{(\Omega R)^{2}} \mathrm{~d} r \\
\mathrm{~d} C_{Q}=\mathrm{d} C_{P}=\frac{\sigma}{2} C_{F_{x}} \frac{U^{2}}{(\Omega R)^{2}} r \mathrm{~d} r
\end{gathered}
$$

Expressions for $C_{F_{z}}$ and $C_{F_{x}}$ are given in Equations 2.33 and 2.34, respectively.

$$
\begin{aligned}
& C_{F_{z}}=C_{L} \cos \phi-C_{D} \sin \phi \\
& C_{F_{x}}=C_{L} \sin \phi+C_{D} \cos \phi
\end{aligned}
$$

From Equations 2.31 and 2.32 it can be seen that thrust and power are dependent 
on the rotor inflow in terms of $U$ and $\phi$ and that knowledge of the inflow distribution across the rotor is essential for rotor performance calculations.

\subsubsection{Blade Element Momentum Theory}

Blade element momentum theory combines momentum theory with a blade element analysis. The spanwise thrust distribution is related to the distribution of induced inflow velocity at the rotor disk, allowing a more realistic estimation of the velocity at the blade section and hence the performance of the rotor as a whole. A rotor in hover or axial flight is considered to operate in an axisymmetric slipstream. The disk is discretized into concentric annuli and the assumptions of basic momentum theory from Section 2.2.1 are applied to each annulus resulting in Equation 2.35.

$$
\mathrm{d} T=2 \rho\left(V_{c}+v_{i}\right) v_{i} \mathrm{~d} A=4 \pi \rho\left(V_{c}+v_{i}\right) v_{i} R^{2} r \mathrm{~d} r
$$

Non-dimensionalizing and realizing $\lambda_{i}=\lambda-\lambda_{c}$ gives Equation 2.36.

$$
\mathrm{d} C_{T}=4\left(\lambda_{c}+\lambda_{i}\right) \lambda_{i} r \mathrm{~d} r=4 \lambda\left(\lambda-\lambda_{c}\right) r \mathrm{~d} r
$$

The blade element theory thrust coefficient equation (Eq. 2.28) can be simplified assuming $U=U_{T}, C_{d} \sin \phi \ll C_{L} \cos \phi$ and $\cos \phi \approx 1$ giving

$$
\mathrm{d} C_{T}=\frac{\sigma}{2} C_{L} r^{2} \mathrm{~d} r
$$

The lift coefficient can furthermore be re-written in terms of the lift curve slope and the zero lift angle of attack using steady linearized aerodynamics [13, p. 119].

$$
C_{L}=C_{l_{\alpha}}\left(\alpha-\alpha_{o}\right)=C_{l_{\alpha}}\left(\theta-\alpha_{o}-\phi\right)
$$


Total inflow ratio in hover can be expressed in terms of $\phi$ and $r$ as follows.

$$
\lambda=\frac{V_{c}+v_{i}}{\Omega R}=\frac{U_{P}}{U_{T}} \frac{\Omega y}{\Omega R}=\tan \phi r \approx \phi r
$$

Assuming that $\alpha_{o}$ can be merged into $\theta$, Equations 2.37, 2.38 and 2.39 can be combined to form Equation 2.40.

$$
\mathrm{d} C_{T}=\frac{\sigma}{2} C_{l_{\alpha}}\left(\theta r^{2}-\lambda r\right) \mathrm{d} r
$$

Combining the momentum equation for the annulus (Eq. 2.36) with the simplified blade element equation (Eq. 2.40) to eliminate $\mathrm{d} C_{T}$ a quadratic equation in terms of inflow ratio is formed with the following solution.

$$
\lambda\left(r, \lambda_{c}\right)=\sqrt{\left(\frac{\sigma C_{l_{\alpha}}}{16}-\frac{\lambda_{c}}{2}\right)^{2}+\frac{\sigma C_{l_{\alpha}}}{8} \theta r}-\left(\frac{\sigma C_{l_{\alpha}}}{16}-\frac{\lambda_{c}}{2}\right)
$$

Hence a blade element momentum theory analysis provides an explicit radial distribution of inflow ratio at the disk without assuming any specific shape or magnitude.

\subsubsection{Blade Element Theory in Forward Flight}

Blade element theory can also be applied to forward flight conditions. Due to the component of edgewise flow, however, the aerodynamic complexity at the blade section is greatly increased. As the flow can no longer be assumed to be axisymmetric, the discretization of the rotor must be extended in the azimuthal direction, requiring azimuth angle $\psi$ - defined zero at the downwind position of the rotor and increasing in the direction of rotor rotation. Due to the asymmetric loading condition of the rotor disk, blade dynamics become a factor and require integration of forces and moments in the time domain. Other complicating effects include unsteady aerodynamics due to changes in 
Mach number, pitch and effective angle of attack, reversed flow near the root of the retreating side of the rotor and interactions with wake structures, which are convected not only downward, but also downwind [13, p. 156]. Extreme occurrences of these effects under high loading conditions eventually lead to dynamic stall and compressibility related drag increases, causing vibration and limiting the performance of the rotor and the operational envelope of the aircraft.

While numerical modelling of these effects is difficult, simplifying assumptions can be made to obtain a first order estimate of the rotor forces using a blade element analysis. The basic aerodynamic considerations for this analysis are summarized here.

Due to the periodic nature of the aerodynamic forces exerted on the rotor blades, hinges or flexures are usually incorporated in the rotor hub design to allow the blades to move in and perpendicular to the rotational plane. The resulting flap and lag motions add new components to the blade element velocity equations (Eq. 2.18 and 2.19) from Section 2.2.2. Equations 2.42 to 2.44 are expressions for the velocities accounting for the situation depicted in Figure 2.4, where the blade is allowed to flap about a hinge on the rotational axis.

$$
\begin{gathered}
U_{P}=V_{c}+v_{i}+r R \dot{\beta}+V_{\infty} \beta \cos \psi \\
U_{T}=\Omega r R+V_{\infty} \sin \psi \\
U_{R}=V_{\infty} \cos \psi
\end{gathered}
$$

The radial velocity component's aerodynamic effect is again neglected due to the sweep equivalence principle ${ }^{2}$. In general, the locations of the flapping and lead-lag hinges may vary with rotor hub design. Specific derivation to account for such variations

\footnotetext{
${ }^{2}$ Leishman states that this component may affect rotor drag [13, pp. 157-158]. Johnson considers radial flow in more detail [30, pp. 213-221].
} 


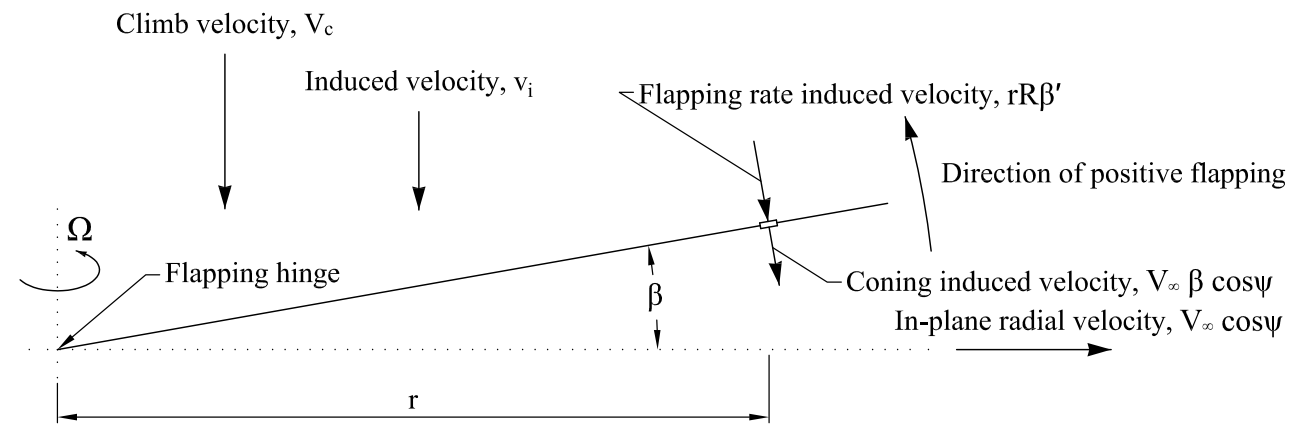

Figure 2.4: Blade flapping and coning induced velocities.

are described in Appendix C.

As the flow through the rotor is no longer axisymmetric, the inflow distribution calculated with the help of momentum analysis at the annulus can no longer be used in forward flight. An accurate treatment of the inflow would require a detailed analysis of the wake, where individual tip vortices cause flow non-uniformities at the rotor disk. Experiments have shown, however, that most of these non-uniformities occur during the transition from hover to forward flight at advance ratios between 0 and 0.1 . At advance ratios above 0.15 the distribution of inflow becomes more linear [13, p. 158].

Numerous models have been developed over the years to approximate the induced inflow at the rotor disk and are documented in Leishman [13, pp. 158-166] and Johnson [30, pp. 134-142]. Initial developments by Coleman et al. and Drees were based on classical vortex theory applications to actuator disk models with an inclined cylindrical wake. Numerical results were approximated with linear variations across the rotor disk in the following form.

$$
\lambda_{i}(r, \psi)=\lambda_{i_{\text {ave }}}\left(1+k_{x} r \cos \psi+k_{y} r \sin \psi\right)
$$

These linear inflow models are also known as first harmonic inflow models. The average induced inflow at the centre of the disk is obtained from the forward flight 
momentum theory equation (Eq. 2.16) and coefficients $k_{x}$ and $k_{y}$ are defined by the inflow model. Leishman provides coefficients for a number of linear inflow models and states that the models of Drees, Payne and Pitt \& Peters ${ }^{3}$ agree best with experimental results [13, p. 160]. Inflow model coefficients for these models and those of Glauert and Coleman et al. are listed in Table 2.1.

Table 2.1: Linear induced inflow model coefficients [13, pp. 159-160].

\begin{tabular}{ccc}
\hline Author(s) & $k_{x}$ & $k_{y}$ \\
\hline \hline Glauert & 1.2 & 0 \\
Coleman et al. & $\tan (\chi / 2)$ & $-2 \mu_{x}$ \\
Drees & $(4 / 3)\left(1-\cos \chi-1.8 \mu_{x}^{2}\right) / \sin \chi$ & 0 \\
Payne & $(4 / 3)\left[\mu_{x} / \lambda /\left(1.2+\mu_{x} / \lambda\right)\right]$ & 0 \\
Pitt \& Peters & $(15 \pi / 32) \tan (\chi / 2)$ & 0 \\
\hline
\end{tabular}

Most expressions for the inflow coefficients are dependent on the wake skew angle $\chi$, which is defined in Equation 2.46 and Figure 2.2.

$$
\chi=\tan ^{-1}\left(\frac{\mu_{x}}{\mu_{z}+\lambda_{i_{\text {ave }}}}\right)
$$

Another inflow model was published by Mangler and Squire in 1950. This model assumes that the pressure difference created across the disk $\Delta p$ - or disk loading - is axisymmetric and can be described by the linear combination of an elliptical distribution and one that vanishes at both the centre and the outside of the disk - denoted Type I and Type III - as shown in Equation 2.47. The nonlinear variation of induced inflow across the rotor disk is given in form of a Fourier series presented in Equation 2.48. Coefficients for the Fourier series are listed in References [13, p. 161] and [36]. The

\footnotetext{
${ }^{3}$ The model developed by Pitt and Peters is a dynamic inflow model [34]. The listed coefficients account only for the static inflow component due to rotor thrust [35].
} 
application of the Mangler and Squire inflow model is limited to advance ratios greater than 0.1 [13, pp. 161-162].

$$
\begin{aligned}
& \Delta p=w_{1} \Delta p_{1}+w_{3} \Delta p_{3}, \quad w_{1}+w_{3}=1 \\
& \lambda_{i}=\left(\frac{2 C_{T}}{\mu_{x}}\right)\left[\frac{c_{0}}{2}+\sum_{n=1}^{\infty}(-1)^{n} c_{n}(r, \alpha) \cos n \psi\right]
\end{aligned}
$$

To move beyond these relatively simple prescribed inflow models requires additional analysis of the wake structures shed by the rotor. Rotor wakes are dominated by strong tip vortices but vortex sheets are also shed along the span of the blade due to spanwise variations in blade loading. Over time these vortices interact with each other, the fuselage and the tail rotor and form very complex wake structures. Vortex wake models track the vorticity in the wake by discretizing it into singularity lines or particles. In steady flight the wake converges to a periodic equilibrium and knowing the vorticity in the wake the induced velocity at the rotor can be obtained using the Biot-Savart law. A large number of vortex filaments are required for an accurate representation of the wake, making this method computationally expensive. Simplifications to free vortex wake models - those where vortex to vortex interactions are calculated - may be to only account for the tip vortices as most of the wake vorticity is concentrated here. Prescribed vortex wake models specify the location of the wake filaments in terms of a wake age parameter based on analytic theory or empirical data.

Computational fluid dynamics (CFD) is seen by many as the eventual goal in accurately solving not only rotor wake aerodynamics but the flow-field around the helicopter in general [13, p. 772], [37]. However, this method is several orders of magnitude more computationally expensive that free vortex models and also methodically difficult to implement: The full Navier-Stokes equations are strongly coupled and highly 
nonlinear, meshing of the rotating blades in a non-rotating environment requires special grid interfacing and finite difference or volume methods tend to dissipate vorticity unrealistically quickly due to numeric round-off. A common simplification is the use of a Reynolds averaged Navier Stokes (RANS) methods where turbulence is modeled empirically. When turbulence and viscous effects can be neglected altogether, the Euler equations may be used.

\subsubsection{Modern Rotorcraft Analysis Tools}

Government research agencies, helicopter manufacturers and academic institutions specializing in helicopter research have developed comprehensive rotorcraft analysis tools to account for the effects of the different rotorcraft disciplines - structures, mechanics, dynamics and aerodynamics - and their interactions on the design and operation of the aircraft. A recent summary of the developments, capabilities and challenges of these programs is provided in Reference [37]. The developments aim to provide a single tool that combines the highest levels of modeling capabilities in the different disciplines balanced by computational feasibility in order to study and improve aircraft performance, vibration levels, control loads, handling qualities, aeroelastic effects, acoustics, etc. To support evaluation of as many aspects as possible, the programs often include various methods to implement a specific discipline at different levels of fidelity. For example, aerodynamic modeling capabilities for calculating the induced velocity at the rotor may include linear inflow models like the ones described in Section 2.2.2.1, dynamic inflow models, prescribed or free vortex wake models. Some programs even include CFD interfaces. In addition, models to account for unsteady aerodynamic, three-dimensional and interference effects may be included in the analysis. 


\subsubsection{Selection of Methodology}

While Carleton University's Rotorcraft Research Group has developed a rotorcraft analysis tool utilizing the discrete vortex wake model of the General Unsteady Vortex Particle software (GENUVP) from the National Technical University of Athens [38], [39] the computational requirements of the analysis were deemed too high for the purpose of an optimization scheme. It was decided that a new lifting-line analysis code be written using lower fidelity aerodynamic models to predict basic rotor performance parameters. For the hover case one-dimensional momentum conservation is used to solve for the induced velocity at the rotor in a manner consistent with blade element momentum theory (see Section 2.2.2.1). For forward flight conditions the prescribed induced inflow models listed in Table 2.1 and the Mangler and Squire induced inflow model were implemented in a blade element theory algorithm. A detailed description of the implemented methods can be found in Chapters 3.2 and 3.3. Considerations regarding the airfoil specific aerodynamic coefficients required for lifting-line methods are discussed in the next section.

\subsection{Airfoil Data Considerations}

Rotorcraft airfoil requirements are different from those used on fixed wing aircraft, because the flow conditions vary drastically in terms of velocity along the rotor span as well as periodically with azimuth in forward flight. Various considerations are discussed in the following sections. 


\subsubsection{Airfoil Aerodynamic Characteristics}

Differences in section and azimuth specific flow conditions are especially obvious in fast forward flight. High Mach numbers are often reached in the tip region of the advancing blades. On the retreating side relatively low dynamic pressures at the blade sections and the appearance of a reversed flow region require high section angles of attack, which may lead to retreating blade stall. Another requirement is a relatively low pitching moment as all torsional loads at the blade root are transferred through the swashplate into the control system. These reasons have lead to specialized development of helicopter airfoils, which depending on their intended spanwise location on the blade contain significant reflex camber at the trailing edge.

\subsubsection{Reynolds Number Effects}

Aerodynamic forces and moments on steady airfoil sections can be non-dimensionalized into functions of angle of attack, Reynolds number and Mach number. Reference [13, pp. 390-391] uses the airfoil maximum lift coefficient as an indicator of the effect of Reynolds number on an airfoil. It is shown that this maximum is relatively constant at Reynolds number above approximately $3 \times 10^{6}$ - where most blade sections of a full sized helicopter operate - and that significant gradients are observed in the $1 \times 10^{6}$ to $3 \times 10^{6}$ range. Since this study is specifically considering $\mathrm{r} / \mathrm{c}$ helicopter main rotors with chord-wise Reynolds numbers largely expected in the range between $1 \times 10^{5}$ to $1.5 \times 10^{6}$, special attention to the effect of Reynolds number on the airfoil is required.

In Reference [16] Carmichael divided the Reynolds number "spectrum" into 12 segments from fractional Reynolds numbers on one end to values greater than $10^{9}$ on the other. The range of Reynolds numbers expected in the current study spans three of Carmichael's ranges: 70,000 to $200,000,200,000$ to 700,000 and 700,000 to 
3,000,000. Considering increasing Reynolds numbers in this range, the difficulties encountered due to laminar separation bubbles decrease as reattachment after the separation bubble is likely to occur at values greater than 200,000 and only minor effects on drag are observed at Reynolds numbers greater than 700,000. Increasing thickness and camber combinations are possible and performance parameters improve considerably with increases in Reynolds number in the specified range.

Experimental low Reynolds number airfoil data references [17], [18], [19] show significant profile drag increases with decreasing Reynolds number especially at values below 200, 000. Higher Reynolds numbers usually result in increases in the stall angle of attack and the maximum lift coefficient.

\subsubsection{Compressibility Effects}

The second non-dimensional parameter affecting steady flow airfoil performance is the Mach number. Due to the velocity difference across the rotor disk, the Mach number also varies drastically. Reference [40] presents experimental airfoil properties for a number of rotorcraft airfoils at Mach numbers from 0.35 to 0.9. Reynolds number is increased approximately proportional to Mach number and depending on data set varies from $4.7 \times 10^{6}$ to $9.3 \times 10^{6}$ or from $0.9 \times 10^{6}$ to $2.2 \times 10^{6}$. Mach number effects include a decrease in the maximum achievable lift coefficient, along with a slight increase in lift curve slope. Drag shows significant increase as Mach number is increased to the drag divergence Mach number, usually located near 0.8. Maximum airfoil normal force to drag ratio also decreases significantly as Mach number is increased beyond 0.5. The effect on pitching moment is small at Mach numbers below the drag divergence Mach number after which they become more negative.

Reference [41] presents experimental data for three helicopter airfoils at different 
Mach numbers and at model scale and full scale Reynolds numbers. Model scale results of two of the three airfoils showed decreased maximum normal force coefficients and all model scale results had increased drag at zero normal force, lower maximum normal force to drag ratios and lower drag divergence Mach numbers for high normal force coefficients with respect to the full scale results. Pitching moment and drag divergence Mach number at zero normal force were insensitive to the Reynolds number changes between the two scales.

\subsubsection{Unsteady Effects}

A further complication of the flow observed at a blade section is that flow conditions are changing so quickly that airfoil characteristics become affected by the rate of change in flow conditions. Most airfoil lift, drag and pitching moment data is collected experimentally in wind tunnels under static or quasi-steady conditions. As the blades flap, lag, pitch, flex, experience different incident velocities and operate in gusts or the wake of other blades, flow conditions vary and affect the lift, drag and moment produced by each blade section. A parameter that is used to determine whether a certain motion can be considered quasi-steady is the reduced frequency $k$ defined in Equation 2.49.

$$
k=\frac{\omega_{k} b}{V_{a v e}}
$$

Reduced frequency is usually defined in terms of the airfoil semi-chord $b=c / 2$. The frequency of the driving variation and the mean velocity are denoted by $\omega_{k}$ and $V_{\text {ave }}$, respectively. If $\mathrm{k}=0$, the flow is steady and up to a value of $\mathrm{k}=0.05$ the flow can be considered quasi-steady. However, in Reference [13, pp. 427-428] Leishman states that even the first order flap frequency of an articulated rotor will have a reduced frequency greater than what can be assumed to be quasi-steady. 
Various methods have been developed to account for unsteady effect. Reference [13, pp. 423-524] provides a summary of different flow conditions that the rotor may encounter, along with research efforts made to model the airfoil response. Major topics include quasi-steady airfoil theory, Theodorsen's theory, wake and gust interactions, time-domain indicial response formulations and varying incident velocities and Mach numbers.

An unsteady aerodynamic effect deserving special mention is dynamic stall. Stall does not occur instantaneously on a rotor blade. Instead as the flow stalls the separated boundary layer rolls up into a vortex, which subsequently grows and rolls over the upper airfoil surface until it is shed at the trailing edge and full separation occurs. While the vortex is travelling along the section surface the airfoil effective angle of attack may continue to increase and may significantly exceed the static stall angle of attack and the corresponding maximum static lift coefficient. After separation it takes time for the airflow to reattach and this usually requires the section angle of attack to be reduced significantly below the static stall angle of attack. Dynamic stall is especially important due to its effect on the airfoil pitching moment. When the vortex is shed at the trailing edge, the pitching moment decreases suddenly. Plotting the moment coefficient for an airfoil in dynamic stall over time shows clockwise and anticlockwise hysteresis loops, which correspond to negative and positive torsional blade damping. Negative damping causes vibrations and can be detrimental to the blade's structure. A selection of empirical models that have been developed to account for dynamic stall are listed in Reference [13, pp. 536-540]. 


\subsubsection{Representation of Static Airfoil Data}

Since the direct calculation of airfoil coefficients during a simulation or optimization run is rarely computationally practical, this data is usually represented in some way as a function of angle of attack, Reynolds number and Mach number. Considering a rotorblade with a rectangular planform and neglecting radial components of velocity, the Reynolds number at a blade section is proportional to the Mach number at the section due to the linear dependency to velocity of each parameter. This allows one to express Mach number in terms of Reynolds number or vice versa.

$$
M=\left(\frac{\mu_{d}}{\rho a}\right) \frac{R e}{c}
$$

The bracketed term depends on the local air properties which are usually assumed constant, while chord length c may vary along the span of the blade, for example, if taper is used. In general, however, chord length will also be relatively constant over a blade region, where a specific airfoil is applied, allowing the aerodynamic properties of the blade to be represented in terms of angle of attack $\alpha$ and one of either Reynolds number or Mach number. Helicopter analysis tools will often use angle of attack and Mach number, since the dependency of airfoil properties on Reynolds numbers in the high Reynolds number range is less pronounced than the dependency on Mach number.

Reference [13, pp. 401-409] lists a number of common approaches to represent static airfoil data. The simplest manner is the use of linear aerodynamic models. Lift is usually specified using the lift curve slope and the zero-lift angle of attack. The drag polar is also approximated using a second order polynomial. A disadvantage of such a simple linear model is that stall effects on lift are not captured. Another approach is direct curve fitting of aerodynamic parameters onto higher order polynomials or ratios 
of polynomials which are able to capture the non-linear behaviour at higher angles of attack. Providing lists of airfoil characteristics in form of look-up tables is a further approach common for large comprehensive rotor analyses and flight simulation models. Coefficients are then interpolated from the tabulated data during the evaluation.

For this study it was decided to represent required airfoil characteristics in look-up table format. Angle of attack and Reynolds number were chosen as interpolation variables to address the low Reynolds number effects encountered in the range of interest for this study.

\subsubsection{Airfoil Data Sources}

In order to assess the influence of different airfoils and their placement along the rotor span, reliable airfoil data is required. Two-dimensional airfoil properties are usually expressed in non-dimensional form as lift, drag and moment coefficients, which can be determined either experimentally using wind tunnel tests or numerically.

Experimental testing is the most reliable and straight-forward way to obtain airfoil coefficients. However, it is a labour and time intensive procedure that requires precise manufacturing of wind-tunnel specimens and access to test facilities.

Experimental airfoil data from early development efforts in airfoil design have been published in well-known references such as [42]. Unfortunately, data is often collected at Reynolds numbers significantly higher than those seen on $r / c$ helicopters of the scale considered in this study. However, a few resources for low Reynolds number airfoil data do exist in the form of the University of Illinois' Summary of Low-Speed Airfoil Data Volumes I to III [43], [44], [17] and the University of Stuttgart's Stuttgarter Profilkataloge $I \& I I$ [18], [19]. These references include experimental results over a large variety of airfoils, usually organized by application. 
The alternative to experimental testing is computational flow simulation. Many computational methods are available to predict the flow around an airfoil. The most reliable form of simulation would be a full computational fluids dynamics (CFD) implementation. In CFD the flow region is divided into a mesh of elements and the governing equations of fluid dynamics - known collectively as the Navier-Stokes equations - are solved numerically throughout the simulation domain. A big advantage of this method is that flow characteristics such as Reynolds number and Mach number can be set to values not easily achievable in experimental conditions. However, due to the non-linearity of the Navier-Stokes equations, these calculations are computationally expensive.

Simplified and faster methods have been developed - mostly based on thin airfoil theory or surface singularity theory - specifically for predicting airfoil performance characteristics. Both theories assume inviscid flow, replace the airfoil shape with strategically placed potential flow singularities and make use of the Kutta condition at the trailing edge. In thin airfoil theory, the flow at the airfoil is represented by a vortex sheet with strengths adjusted to make the camber line a streamline of the overall flow [45, pp. 298-306]. Surface singularity or panel methods replace sections of the airfoil surface with flow singularities like vortices or sources and control point constraints ensure that the streamlines are parallel to the airfoil surface [45, pp. 319-325].

Since both of these methods are based on an inviscid flow assumption, they are usually coupled to a boundary layer flow solver to account for laminar flow, transition and turbulent flow as well as separation and potential reattachment of the boundary layer, all of which greatly affect the viscous drag experienced by the airfoil [46]. Difficulties with such viscous-inviscid interaction methods include the prediction of laminar to turbulent flow transition, handling of separation bubbles and the fact that the boundary layer equations break down when flow separation occurs and the thickness of the 
boundary layer increases drastically. Various models may be used in an attempt to capture these effects.

Tinis [46] recently developed a viscous-inviscid interaction airfoil analysis tool at Carleton University that will be referred to as VII in the remainder of this document. VII is based on a two-dimensional source-vortex panel method and the momentumintegral method for the inviscid and viscous portions, respectively, and includes a new model to predict transition in separated and attached boundary layers [47]. Another well known viscous-inviscid interaction method for airfoil analysis and design is Drela's XFOIL released under the GNU general public license [48]. XFOIL utilizes an inviscid linear vorticity panel method and a viscous lagged dissipation integral method. The software has been shown to be well suited for low Reynolds number applications [49].

\subsubsection{Selection of Airfoil Parameter Generator}

While computational fluid dynamics was identified as the most reliable numerical method to generate airfoil characteristics, evaluations of a large number of airfoils over a range of $\mathrm{r} / \mathrm{c}$ helicopter flight conditions was left as a future task in the interest of continued focus on the objective of this work. Tinis' VII software was initially chosen as the two-dimensional properties generator as it was a Carleton University developed package and included recent developments for separation bubble effects. Since the tool was new, however, it was tested on a number of airfoils, for which published experimental data in the Reynolds number range of interest was available. During this process it was identified that the treatment of flow separation was not mature and results at angles of attack approaching stall were unsatisfactory. Section 3.1.1 lays out the efforts made to create an empirical model to enable the prediction of airfoil lift stall characteristics in order to correct for this deficit. Due to difficulty in obtaining the desired level of 
accuracy from the VII algorithm, its results - along with the empirical lift stall estimation - were compared to experimental data and XFOIL results for a variety of airfoils in the Reynolds number range considered in this study. Figures of this comparison are included in Appendix A. As discussed in Section 3.1.2, XFOIL was finally selected as the more reliable airfoil data generator.

\subsection{Optimization Applied to Rotorcraft Design}

Two recent surveys on the application of optimization in the field of rotorcraft design are provided by Celi [50] and Ganguli [51]. Both authors focus on optimization techniques applied to the heavily multidisciplinary areas of aeromechanical rotor effects, design of composite rotor blades, flight mechanics and handling qualities. The most common goal was the minimization of hub vibratory loads, but weight, noise and rotor performance were also part of the objective functions of several of the presented studies. With respect to optimization techniques the studies are generally divided into two groups: utilizing traditional gradient based algorithms or heuristic methods such as genetic algorithms or simulated annealing.

The authors of References [50] and [51] both comment that the predictions obtained from even the most sophisticated rotor aeroelastic analysis codes are poor, which limits the accuracy of any optimized design. Celi [50] also states that experimental verification of whether the optimized design is truly optimum is most often impractical as such a confirmation would require building and testing of a number of designs close to the numerically generated optimum. Therefore, verification that the application of optimization techniques lead to an improved design is usually limited to a comparison of the final design and a baseline configuration. All numerical studies which were confirmed 
in this way showed an improvement due to the changes suggested by the optimization routine.

\subsubsection{Optimization Methods}

The optimization efficiency of gradient based methods depends on the ease of providing or estimating the sensitivity of the objective function to the design parameters. Due to the multidisciplinary nature of many rotorcraft optimization problems, this gradient information is difficult to obtain. Finite difference approximations are often a large draw on efficiency as they require a large number of additional function evaluations and their accuracy depends on the perturbation step size. Analytical or semi-analytical gradients can be developed to bypass these difficulties; however, extensive modification to the underlying analysis computer programs are often required. Another weakness of gradient based methods is the fact that they tend to "get stuck" in local minima of the objective function and therefore do not guarantee that the optimized design presents a global optimum. It is suggested that multiple initial conditions be used when using these methods to increase the likelihood of finding the true optimum. Non-convex design spaces will limit convergence efficiency and discrete design parameters cannot easily be accommodated by gradient methods.

Typical gradient based optimization techniques include the method of steepest descend and Newton's method [52, pp. 113-184]. The method of steepest descend only requires first order derivatives of the objective function with respect to the design variables. In each iteration of the algorithm the direction of steepest descend (towards the minimum of the objective function) is determined from the gradient information. Then a line search method is applied to find the minimum of the objective function along this 
direction. The process is repeated by recalculating gradients at the new location. Newton's method approximates the curvature at the current design with quadratic functions which can easily be minimized mathematically. However, the Hessian - a $n \times n$ matrix of second derivatives of the objective function relative to $n$ design variables - is now required. Due to this added complexity quasi-Newton methods and conjugate methods have been developed to approximate the Hessian or its inverse using first derivative gradient information only.

Heuristic optimization methods based on genetic algorithms or simulated annealing do not depend on gradients and are also denoted global optimization methodologies as they are more likely to find the absolute optimum position in the design space. Genetic algorithms start with a population of designs composed of a random arrangement of design variables represented in a string - most often using binary format. From one "generation" to the next design points are selected based on their "fitness" and genetic operations of crossover and mutation lead to a new population, where improved traits prevail [52, pp. 237-249]. As the design variable information can be stored in binary form genetic algorithms are specially suited to discrete variables. However, representing a large number of continuous design variables of significant precision quickly increases the length of the bit string, which is counterproductive to the algorithm efficiency.

Simulated annealing methods follow the physical processes observed during metal or crystal solidification. Reference [50] describes the following sample implementation: First, the objective function for the current design is calculated. Then the design is perturbed and the objective function is recalculated. In the third step the perturbed design is accepted as the new design, if the objective function improved or a randomly generated condition is satisfied. As the optimization progresses, the probability of meeting 
the random conditions is reduced. Optimization algorithms based on simulated annealing are able to handle discrete variables provided that the perturbation rules are designed respectively. The likelihood of finding the global optimum even in non-convex design spaces with local minima is improved by the random condition in the selection process.

Celi [50] predicted in 1999 that gradient based methods would remain dominant in rotorcraft related optimization for some time, while methods like genetic algorithms and simulated annealing would be reserved for discrete design variables and preliminary global design space searches. In 2003, Ganguli [51] reported a significant increase in the utilization of gradient-free optimization methods, but concludes that many different methods are still used and merited for various applications.

\subsubsection{Aerodynamic Rotor Performance Optimization Studies}

The following paragraphs summarize a number of rotorcraft design optimization applications either singly considering or otherwise placing great importance on rotor aerodynamic performance.

In the early 1990's, NASA placed special focus on the development of multidisciplinary design procedures. In Reference [53] the aerodynamic performance optimization of a four bladed $2.75 \mathrm{~m}$ diameter rotor is presented. Rotor performance in hover was determined using the strip theory momentum analysis program HOVT. Two forward flight conditions were simulated using the comprehensive helicopter analysis program CAMRAD, one representing a cruise condition at an advance ratio of 0.3 and the other indicative of a manoeuvre at a load factor of 1.2. CONMIN, a general purpose optimization program, was used as the optimization algorithm along with a finite difference sensitivity analysis. Four design variables were used: the point of taper initiation, root 
chord, taper ratio and linear twist rate. The airfoil selection was predetermined. Constraints included limits on power and drag coefficient magnitudes to retain a healthy margin from the stall point and the drag divergence Mach number of the airfoil, a minimum tip chord length and a condition to ensure that the rotor system could be trimmed at the three flight conditions. The objective function was defined as the minimization of power required during hover. Two different cases for the forward flight analyses were conducted. The first assumed a uniform inflow distribution, while the second included a prescribed rigid wake. The two cases returned significant differences in the taper initiation point. The optimized blades produced reductions in both hover and forward flight power, while the power required for the manoeuvre condition increased in most cases. It was noted that the optimized design operated significantly closer to stall compared to the reference design.

Reference [54] built on the developments of Reference [53]. In this paper the aerodynamic optimization is coupled with a dynamic analysis monitoring hub loads and adjusting tuning masses to prevent overlapping of the blade elastic frequencies with integer multiples of rotor speed. HOVT and CAMRAD/JA were used for the analysis of hover and forward flight conditions, respectively. CAMRAD/JA also provided the blade frequencies and hub reactions. CONMIN was used as the optimization algorithm. The aerodynamic design variables listed above for Reference [53] are retained. A total of fifteen dynamic design variables were added, nine represented chordwise, flapwise and torsional stiffnesses at three radial locations and the remaining six were reserved for three tuning masses and their locations. The objective function was expanded to include the minimization of a linear combination of the power required in hover, forward flight and the manoeuvre condition, as well as the four-per-rev vertical hub shear stresses. In addition there were 54 performance constraints and 26 dynamic constraints. Several 
optimization cases were conducted considering hover, forward flight and manoeuvre power requirements or hover exclusively. The integrated optimization of aerodynamic performance and dynamic design variables was also compared to a sequential optimization scheme - first with respect to aerodynamics and second with respect to dynamics. The integrated optimization approach produced a superior design both in terms of performance and dynamic behaviour for the cases where forward flight and manoeuvre power were included in the objective function. Excluding these two parameters from the objective function resulted in inferior performance measures from the optimum design returned by the integrated approach compared to that of the sequential approach. However, the integrated design provided significantly lower vertical hub shear loads in the forward flight condition.

A multilevel decomposition procedure for rotorblade optimization is described in Reference [55]. The procedure considers a multidisciplinary optimization of rotorblade dynamics, aerodynamics, structure and aeroelasticity and divides the optimization into three levels, each focusing on a single discipline. First, the rotor is optimized for aerodynamic performance, adjusting the blade chord and twist distributions to minimize power required in forward flight at an advance ratio of 0.3 , while holding thrust constant. At level two, dynamics and aeroelastic effects are evaluated and blade stiffness distributions and non-structural blade tip weights are adjusted to minimize the dominant hub shear forces. Last, the wall thicknesses of the blade box beam are adjusted to meet the stiffnesses obtained in level two, while minimizing the total blade mass. The different levels are coupled only through constraints and the optimization was conducted by "cycling" through the three levels multiple times. To accommodate the minimization of multiple shear forces in the second level the Kreisselmeier-Steinhauser function approach was used to reformulate multiple objectives and constraints into a 
single objective function. CAMRAD was used for the blade aerodynamic, dynamic and aeroelastic stability analyses, while an in-house code was used to analyse the structure of the box beam. CONMIN was used as the optimization scheme. The procedure was observed to efficiently produce an optimum design with improvements noted with respect to each discipline.

A more recent aerodynamic helicopter rotor optimization study is presented in Reference [56]. A lifting line method based code is used to calculate the blade deformations at each optimization step. The deformed geometry is then analyzed in the ONERA developed 3D Reynolds averaged Navier Stokes solver elsA. Only hover is considered. CONMIN is again used to perform the optimization. Available design variables included chord, sweep, anhedral and twist distributions as well as airfoil positions. Collective pitch was also added to the design variables to enable changes in thrust coefficients in order to maximize the rotor figure of merit selected as the objective function. The paper presents optimizations performed with respect to individual design variables, demonstrating the resulting performance effects. Finally, an optimization with respect to eight design variables representing chord, anhedral, sweep and twist along the rotor blade was performed starting from a blade geometry (previously) designed for low noise operation. Considerable performance improvements were predicted for the optimized design. The planform shape of the optimized rotor was significantly different from the baseline design; no comment was made on whether the low noise characteristics were retained.

In Reference [28] a procedure used to optimize micro aerial vehicle and nano aerial vehicle rotor hover performance is presented. As mentioned in Section 2.1 the Reynolds numbers that rotors of this scale operate in are very low $(5000 \leq R e \leq 60000)$ and the study was therefore limited to a family of thin circular arc airfoils. The aerodynamic 
performance analysis was conducted using a BEMT algorithm with 2D CFD generated airfoil coefficient data. The optimization was divided into two steps. First, a grid search of the design space was used to determine the approximate location of the optimized design within initial constraints. Starting from the point with the highest power loading factor the second step applied a constrained nonlinear optimization procedure - coded in MATLAB - to maximize power loading in the neighbourhood of the result of the first step. The optimized rotor geometry for a $6 \mathrm{~g}$ helicopter with a $7.5 \mathrm{~cm}$ diameter rotor is presented along with experimental results.

\subsubsection{Optimizer Selection}

Since the two rotor performance simulation modules developed in this work were written in MATLAB, the MATLAB Optimization Toolbox was selected to provide an optimization algorithm. The toolbox includes various gradient-based and heuristic solvers [57]. Both simulation modules were developed to operate with manageable computational cost, which limits the penalty obtained due to repeated function evaluations for the generation of finite difference gradients. Therefore, the gradient-based solver fmincon was chosen for the optimization tasks presented in this work. fmincon is designed to find the minimum of nonlinear multivariable functions subject to bounds on the design variables and linear or non-linear equality and inequality constraints in terms of the design variables. The implementation of the hover module simulation with this optimizer is presented in Chapter 4. 


\section{Chapter 3}

\section{The Qoptr Simulation Modules}

The quick rotor optimization framework Qoptr was developed to achieve the objective of this thesis. While a lot of time and effort went into the development of a practical, fast and user-friendly engineering tool, this chapter will focus only on the theoretical aspects in the software that relate to the performance simulations.

The Qoptr framework contains two simulation modules:

- The hover module uses a blade element momentum theory approach to determine the induced inflow distribution. This method assumes axisymmetric flow conditions and hence is applicable to rotors in hover and axial climb.

- The forward flight module is based on blade element theory. Non-zero advance ratio conditions can be simulated using prescribed inflow models to define the induced inflow distribution at the rotor disk. The module includes fully articulated as well as teetering rotor hub models to allow rigid blade motion.

Both of these simulation modules rely on airfoil data that is organized into look-up tables as a function of Reynolds number and angle of attack. The VII program and XFOIL are therefore evaluated in Section 3.1 on their ability to generate reliable airfoil data in the low Reynolds number regime applicable to 700 class electric $r / c$ helicopters. 
Sections 3.2 and 3.3 then detail the main iteration loops of the simulation modules. Simulation results are validated through comparison to published experimental data in the respective sections.

\subsection{Generating Two-Dimensional Airfoil Properties}

This section details the main problem encountered with airfoil coefficient estimations provided by the VII algorithm [46], the subsequent efforts made to develop an empirical airfoil lift stall correction model and the final decision made to use XFOIL as the tool to generate two-dimensional airfoil data. Implemented airfoil data corrections for compressibility effects and limitations of the current implementation are also summarized.

\subsubsection{Lift Stall Correction for the VII Algorithm}

Tinis's VII algorithm was introduced in Section 2.3.3. As this tool was only recently developed, its results for a number of airfoils were compared to published experimental data in the Reynolds number range of interest. It was identified that the treatment of flow separation was not able to predict airfoil characteristics properly once the flow separated from the upper surface without reattachment, unless an empirical free-shear layer angle coefficient $m$ was provided to define the angle at which the viscous shear layer separates from the airfoil surface [46]. The only solution provided to define this factor $m$, which would vary from one airfoil to the next and also change with angle of attack and flow condition, was reverse derivation from experimental data. Since effects due to the onset of stall could not be practically determined, VII by itself was deemed not suitable to provide reliable airfoil data at high angles of attack. In an attempt 
to "correct" the lift coefficient polar in the stall region an empirical model based on experimental data at the Reynolds number range of interest was developed as described in the next section.

\subsubsection{Evaluation of Experimental Data}

Using data presented in Summary of Low-Speed Airfoil Data, Vol.3 [17] and corresponding airfoil profile coordinates from Reference [58] a database containing the following parameters was constructed.

1. Airfoil thickness to chord ratio

2. Airfoil camber relative to chord length

3. Maximum airfoil upper surface deflection from the chord line

4. Estimated leading edge radius ${ }^{1}$ relative to chord length

5. Reynolds Number

6. Maximum lift coefficient

7. Angle of attack corresponding to the maximum lift coefficient

8. Angle of attack corresponding to stall onset, approximated by the deviation in the lift polar.

9. Stall shape factor. A numeric value assigned to the severity or 'sharpness' of the observed stall behaviour relative to the change in angle of attack.

\footnotetext{
${ }^{1}$ The validity of the estimation used to determine the airfoil leading edge radius was never confirmed.
} 
Reference [17] contains wind-tunnel measurements for a variety of airfoils developed mostly for $\mathrm{r} / \mathrm{c}$ powered and unpowered fixed wing and wind turbine applications at Reynolds numbers from 60,000 to 500,000 . The correlation database was limited in Reynolds number to tests performed between 100,000 and 500,000. Furthermore, only data from laminar - not intentionally tripped - tests and conventionally ${ }^{2}$ shaped airfoils were included in the correlation database.

The data set was examined graphically for possible correlations between stall parameters and airfoil shape and flow characteristics. Only the maximum lift coefficient and its corresponding angle of attack revealed promising results. The maximum lift coefficient showed reasonable correlation to Reynolds number and camber, while the angle of attack at maximum lift coefficient indicated dependence on Reynolds number and the maximum upper surface deflection. No convenient correlations were observed for the stall onset angle of attack and the shape factor.

In order to increase the Reynolds number range of the experimental data beyond 500,000, Stuttgarter Profilkatalog I \& II [18], [19] and their data were purchased. These data sets contain experimental wind-tunnel results acquired at Reynolds numbers ranging from 300,000 to $3,000,000$. Again selecting data only from laminar tests the correlations for maximum lift coefficient and the corresponding angle of attack were examined. Satisfactory correlation was observed for the maximum lift coefficient. However, the correlation to the angle of attack at maximum lift coefficient was poor. To obtain an acceptable result the Stuttgart data set was limited further to results with a maximum Reynolds number of 1,500,000 and airfoils exhibiting significant airfoil stall

\footnotetext{
${ }^{2}$ Airfoils resembling curved flat plates and special interest airfoils were excluded.
} 
followed by lift coefficient increase over a prolonged angle of attack ranged ${ }^{3}$ were excluded. In addition, the locations of the maximum airfoil thickness, camber and uppersurface deflection along the chord of the airfoil were added as independent parameters. A complete list of independent and dependent parameters used in the correlation is presented in Table 3.1.

Table 3.1: Lift stall estimation correlation parameters.

\begin{tabular}{ccc}
\hline Parameter & Abbreviation & Dependency \\
\hline \hline Thickness & $t / c$ & \\
Camber & $c a m / c$ & \\
Maximum Upper-surface Deflection & $y_{\max } / c$ & Independent \\
Reynolds Number & $R e$ & Parameters \\
Location of Maximum Thickness & $x_{t}$ & \\
Location of Maximum Camber & $x_{c a m}$ & \\
Location of Maximum Upper-surface Deflection & $x_{y_{\max }}$ & \\
\hline Maximum Lift Coefficient & $C_{l_{\max }}$ & Dependent \\
Angle of Attack at Maximum Lift Coefficient & $\left.\alpha\right|_{l_{l_{\max }}}$ & Parameters \\
\hline
\end{tabular}

A MATLAB script - utilizing linear correlation techniques from Reference [59] - was used to determine the correlation of the dependent parameters with respect to the independent parameters in more detail. Parameters exhibiting comparatively weak correlations to the dependent variables or strong correlations to other independent variables were excluded to determine the final trends shown in Equations 3.1 and 3.2.

\footnotetext{
${ }^{3}$ See for example lift polars for airfoils FX 74-130 WP1, FX 74-130 WP2, AH 82-150F, AH 81-131, etc. in Ref. [19]
} 


$$
\begin{aligned}
& C_{l_{\max }} \approx 0.2852+9.737^{\mathrm{cam} / c}+9.288 \mathrm{t}_{\mathrm{c}}^{\mathrm{t}}+0.2857 \mathrm{x}_{\mathrm{cam}}+57.99 y_{\max }{ }^{2} \\
& -0.4436 x_{\mathrm{cam}}{ }^{2}+12.90 \mathrm{cam} / \mathrm{c} / \mathrm{c}-90.05 \mathrm{t} / \mathrm{c} y_{\max } / \mathrm{c}+2.705 \times 10^{-5} \mathrm{cam} / \mathrm{c} R e \\
& +1.5835 \times 10^{-5 t} / c R e-2.949 \times 10^{-5} y_{\max } / c R e \\
& \left.\alpha\right|_{C_{\operatorname{lmax}}} \approx-3.655+100.7{ }^{\mathrm{t}} / \mathrm{c}+212.5^{y_{\max } / c}-3.904 x_{\text {cam }}+9.297 x_{\text {cam }}{ }^{2} \\
& -12611^{t} c^{y_{\max } / c}-6.014 \times 10^{-5 \mathrm{cam} / c} \mathrm{Re}+1.138 \times 10^{-5} y_{\max } / \mathrm{c} R e
\end{aligned}
$$

These relationships resulted in correlation coefficients of 0.91 and 0.61 for the maximum lift coefficient magnitude and its corresponding angle of attack, respectively. Histograms of the error in the estimated values of the two parameters are shown in Figure 3.1. The standard errors in the estimated lift coefficient value and its angle of attack were 0.121 and $1.75^{\circ}$, respectively. 


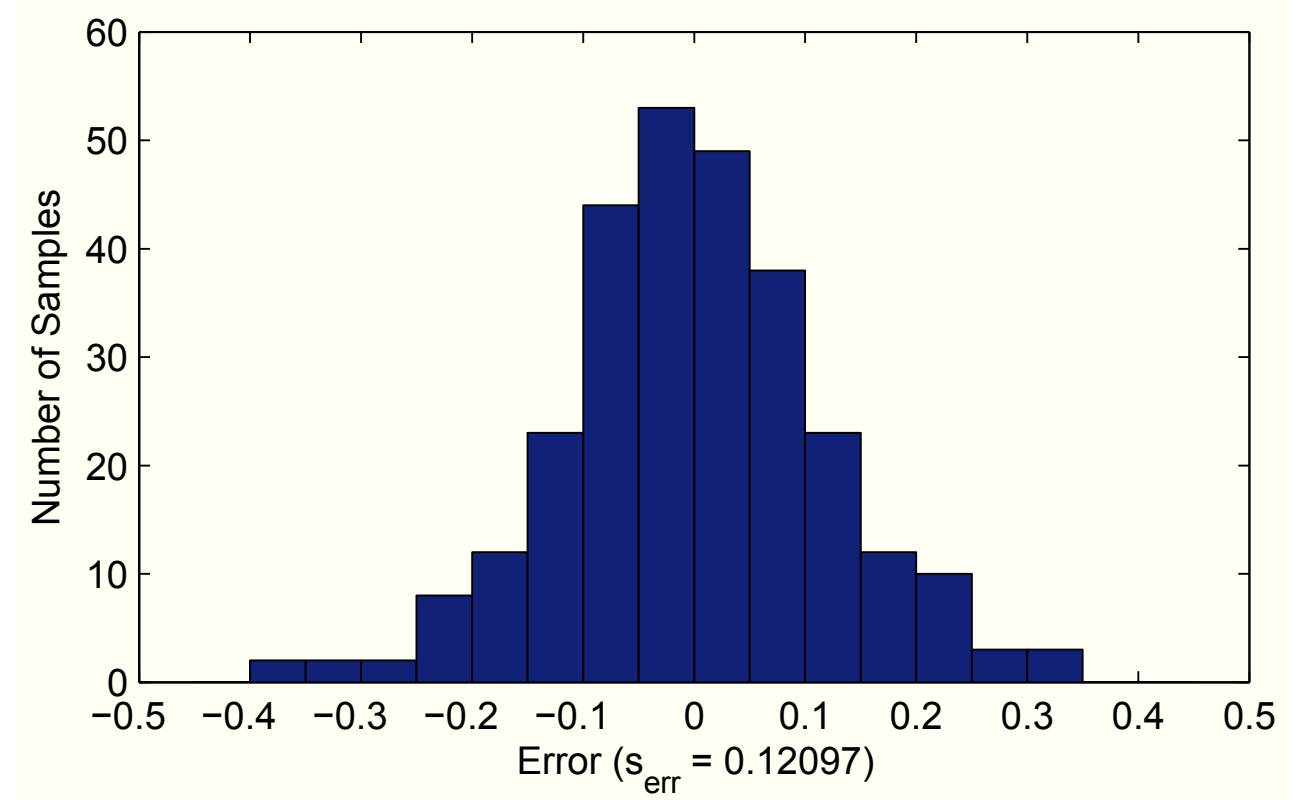

(a) Correlation error in maximum lift coefficient $C_{l_{\max }}$.

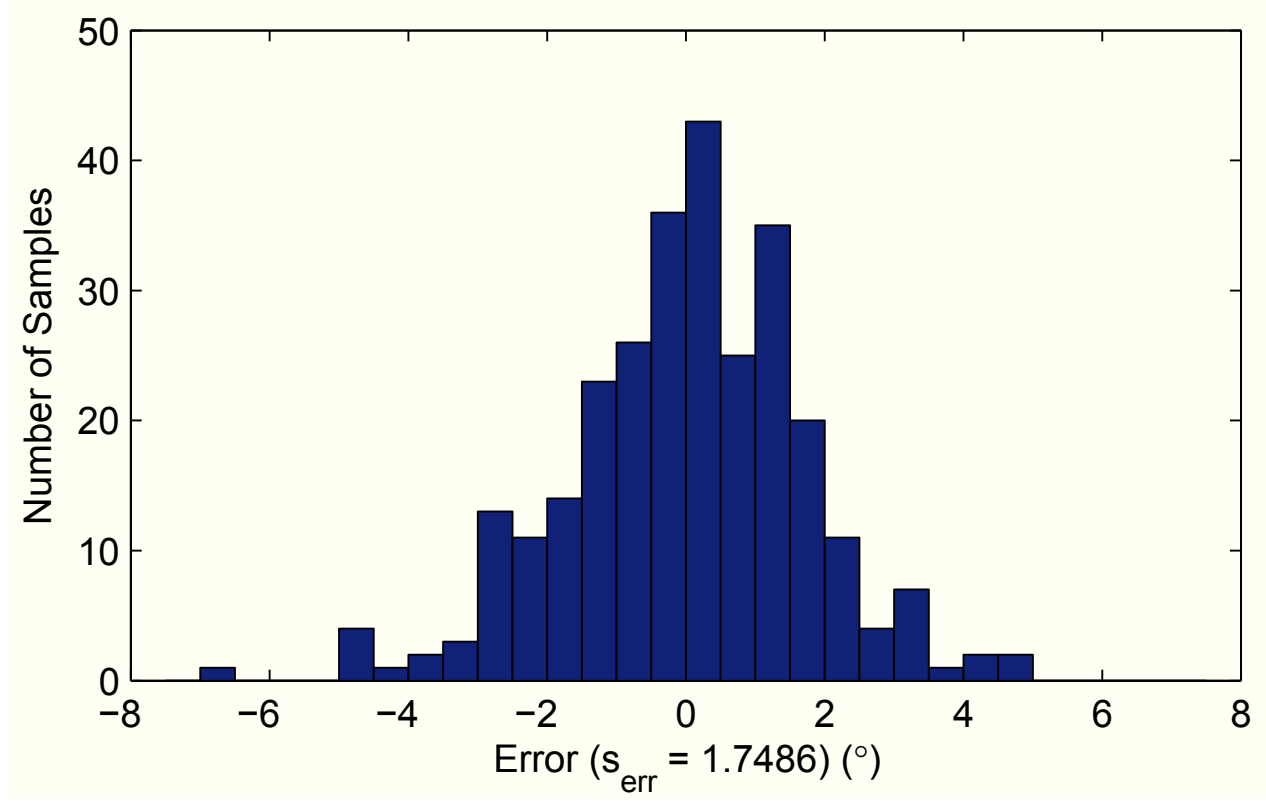

(b) Correlation error in maximum lift angle of attack $\left.\alpha\right|_{l_{\operatorname{lmax}}}$.

Figure 3.1: Histograms of error in the estimated lift coefficient maximum point coordinates on the lift polar described by Equations 3.1 and 3.2. 


\subsubsection{Lift Stall Estimation Model}

Given the observed trends for the maximum lift coefficient point on the lift polar from Equations 3.1 and 3.2, a sensible way of combining this estimate with the results of the VII algorithm was required. It was decided that a downward facing parabola would be used for this purpose as its shape agrees with the general behaviour of an airfoil near stall and it can be easily specified using only two conditions. The parabola was defined by enforcing tangency to the VII lift curve and by specifying the vertex to lie at the estimated maximum lift coordinates. Mathematically, the problem is shown in Equations 3.3 and 3.4 .

$$
\begin{gathered}
C_{l}=a_{c}\left(\alpha-\left.\alpha\right|_{C_{l_{\max }}}\right)^{2}+C_{l_{\max }} \\
a_{c}=\frac{C_{l_{\alpha}}{ }^{2}}{4\left(C_{l_{\max }}-\left.\alpha\right|_{C_{l_{\max }}} \times C_{l_{\alpha}}-\left.C_{l}\right|_{(\alpha=0)}\right)}
\end{gathered}
$$

For the purpose of defining the point of tangency, the VII results were approximated by a linear fit to the curve below the estimated maximum angle of attack ${ }^{4}$. This linear fit provided constants for the lift curve slope $C_{l_{\alpha}}$ and the intersection with the vertical axis $\left.C_{l}\right|_{(\alpha=0)}$. The coordinates of the point of tangency between the line of best fit and the parabola can be determined as shown in Equations 3.5 and 3.6.

$$
\begin{gathered}
\alpha_{x}=\alpha_{\max }+\frac{C_{l_{\alpha}}}{2 a_{c}} \\
C_{l_{x}}=C_{l_{\alpha}} \times \alpha_{x}+\left.C_{l}\right|_{(\alpha=0)}
\end{gathered}
$$

All of these functions were integrated into a MATLAB script to streamline the procedure of applying the lift stall estimation to a data set returned by the VII package. As an example the parabolic stall prediction for the "Great Planes PT40" airfoil at a

\footnotetext{
${ }^{4}$ This seemed reasonable as the VII algorithm produces a nearly straight lift curve, when the free-shear layer angle coefficient is given a value of $m=0$.
} 
Reynolds number of 400,000 is shown in Figure 3.2. Experimental data is taken from Reference [17].

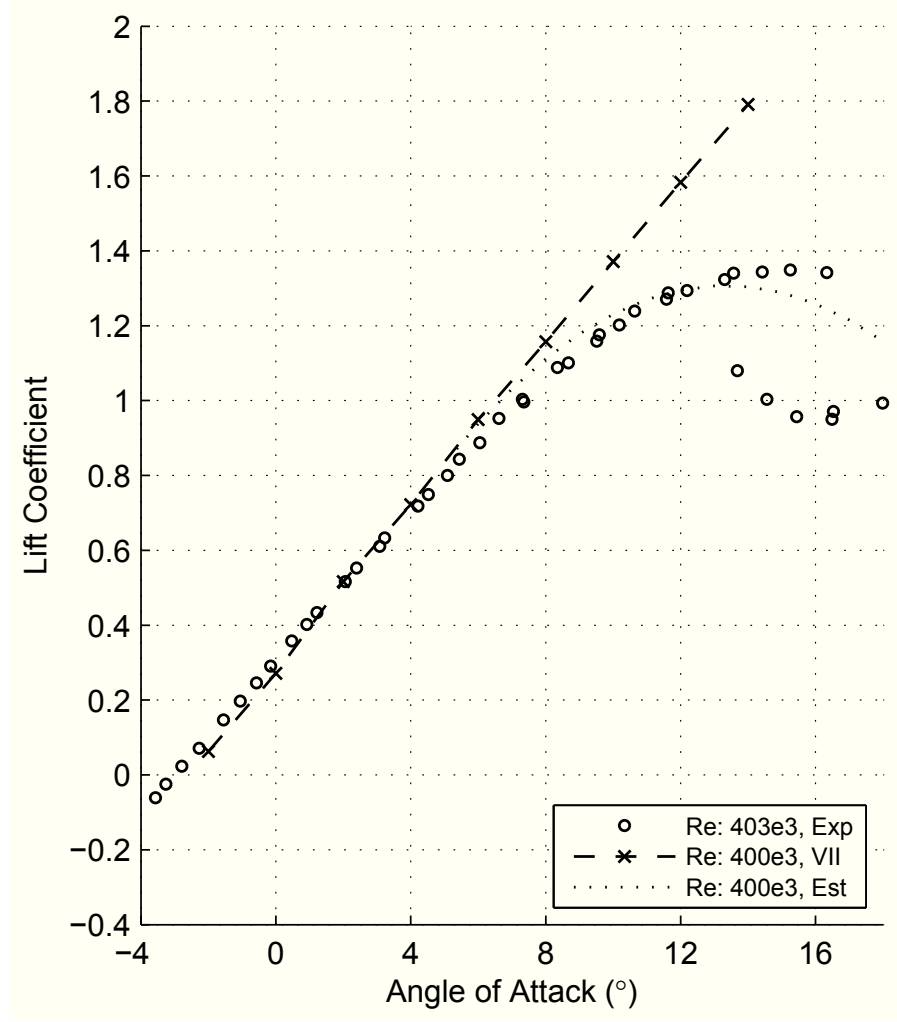

Figure 3.2: Great Planes PT40 airfoil lift coefficient polars showing the VII output $(m=0)$ and the empirical stall estimate at $R e=400,000$. (Experimental data from Ref. [17].)

\subsubsection{Limitations of the Lift Stall Estimation}

As presented in Figure 3.1, the accuracy of the predicted maximum lift coefficient point is limited and - considering two standard deviations - the predicted point may be $\pm 3.5^{\circ}$ and \pm 0.24 from the true maximum in angle of attack and lift coefficient magnitude, respectively. Therefore, not every airfoil is expected to agree with the trend as well as the airfoil pictured in Figure 3.2. One such example is the Eppler E472 airfoil for which the lift polars at two Reynolds numbers are shown in Figure 3.3. While the estimated lift 
curves show an increase in the maximum lift coefficient with the increase in Reynolds number, the experimental results show a much greater performance difference between the two flow conditions.

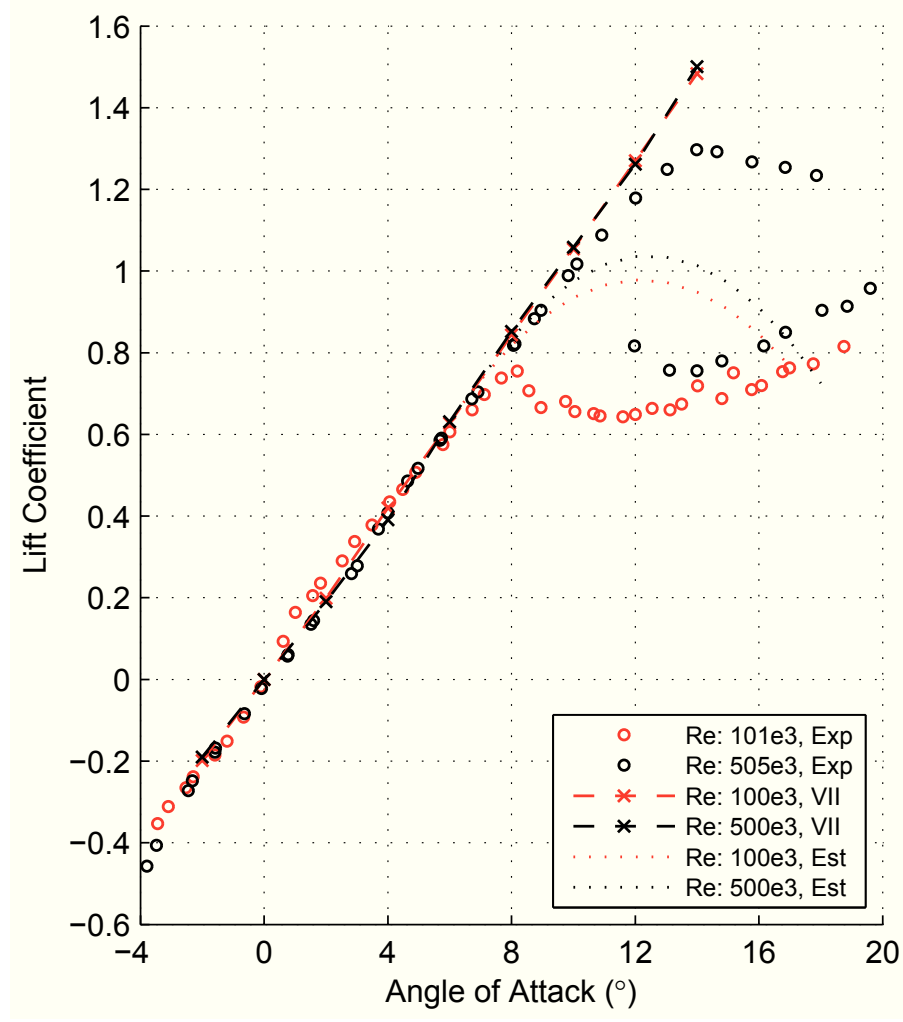

Figure 3.3: Eppler E472 airfoil lift coefficient polars showing the VII output $(m=0)$ and the empirical stall estimate at $R e=100,000 \& 500,000$. (Experimental data from Ref. [17].)

A further limitation of the presented lift stall model is that there is no guarantee that the shape of the real lift polar at stall is well approximated by the implemented quadratic function. Defining the parabola by the position of its vertex and tangency to a line causes a more sudden stall behaviour if the predicted maximum lift point is situated close to the line predicted by VII; alternatively, if the distance between the line and the vertex is large, the stall behaviour appears more gradual. This effect can 
be observed in Figure A.2f of Appendix A. The lift polar stall behaviour beyond the maximum lift condition often exhibits abrupt changes or other non-linearities. These are also not captured by the parabola shape, though by definition a decreasing trend will be predicted.

It should also be noted that the model was developed strictly from experimental data collected at low Mach numbers and that no attempt was made to correct the VII predicted drag and moment coefficients near stall.

\subsubsection{Validation of Two-Dimensional Airfoil Properties}

Appendix A contains a number of figures showing the application of the VII algorithm corrected with the lift stall estimation model presented in Section 3.1.1. Experimental data from References [17], [18] and [19] is included to allow an assessment of the overall performance of the tool in generating airfoil lift, drag and moment coefficients.

In Reference [46] Tinis states that airfoil lift coefficients can be predicted well into the stalled region specifying $20^{\circ}$ angle of attack as a limit for the S809 airfoil at Reynolds numbers $1 \times 10^{6}$ and $2 \times 10^{6}$. The airfoil showed significant stall effects on the lift coefficients initiating at about $7^{\circ}$ angle of attack. Due to the lack of a practical method to define the free shear layer angle factor $m$, these claims could not be confirmed during the tests conducted for the present study. Trials were performed assuming varying constant values for $m$, but no solution applicable for a range of airfoils was identified. Therefore, a value of zero was assumed and the estimated lift stall model was implemented to correct the lift polar at stall. At angles of attack below stall the lift curve produced by the VII algorithm agrees relatively well with the experimental data, though in some cases unexplained outliers were observed at individual angles of attack. The lift stall estimation model also performed relatively well within the limits of 
the correlation, however, as already discussed in Section 3.1.1.3, the accuracy of the statistical prediction was low and the shape of the lift stall behaviour could at best be used up to a few degrees angle of attack beyond the maximum lift point. Some tests showed significant offsets between the predicted lift polar and the experimental one. Thin airfoils seemed to perform best in this regards.

With respect to drag and moment coefficients Tinis [46] states that drag is likely under-predicted and pitching moment over-predicted at angles of attack beyond stall. These trends were mostly confirmed in the results shown in Appendix A. While drag coefficients showed the expected decrease with respect to Reynolds number increases, zero lift drag coefficients at Reynolds numbers below 200, 000 were significantly underpredicted. At higher angles of attack the increase of drag coefficients did not match the experimentally observed effects. This may again have been caused by the fact that the shear layer angle factor was unknown and assumed to be zero. The intervals of angle of attack chosen to produce the figures in Appendix A were relatively large at $2^{\circ}$ because the algorithm requires time on the order of a minute to produce results for a single data point. While this caused rather poor resolution, the VII results did not seem to capture more intricate drag coefficient details like the "drag buckets" shown in the experimental data polars of Figures A.2a to A.2c.

Pitching moment magnitudes predicted by VII at low angles of attack were generally fair, while the accuracy decreased with increasing amount of camber. Variations with angle of attack were not represented well compared to the experimental data and stall effect were not captured.

Due to the limited accuracy of the VII results to experimental lift and moment coefficients and significant deficits in the drag coefficient matches the airfoil comparison was repeated using XFOIL [48]. The resulting polars are also shown in Appendix A. While 
the matches of some lift polars seemed worse that those produced by the corrected VII algorithm, most were comparable in accuracy. The XFOIL drag vs lift curves outperformed the VII algorithm for every airfoil in the test series and non-linear trends like the aforementioned drag buckets were reproduced very well. Moment coefficients were predicted to a comparable or better accuracy with respect to the VII results. Compared to the experimental data, XFOIL often predicted higher maximum lift coefficients, and drag coefficients were in general underpredicted. Several of the drag versus lift coefficient plots in Appendix A show a considerable horizontal offset between the experimental and XFOIL data lines at high lift coefficients. This causes a significant underprediction in drag coefficient towards higher angles of attack, which was also noted in References [60]-[62].

A clear advantage of XFOIL is the speed of the algorithm. Results for a complete range of angles of attack can be computed in seconds allowing for higher angle of attack resolution without great time penalties. During the XFOIL data runs a number of airfoil flow conditions were not able to converge, especially at higher angles of attack, where this is expected due to severe separation of the boundary layers. The graphical output provided convenient and quick feedback when such conditions were encountered.

As the two-dimensional data is to be used to predict rotor performance parameters, the significantly superior drag coefficient matches as well as comparable lift and moment coefficient predictions lead to the decision to choose XFOIL as the airfoil analysis tool for this study. Faster execution times and a well developed user interface were a bonus that also fit well into the overall objective of developing a fast optimization tool. 


\subsubsection{Compressibility Corrections for Two-dimensional Airfoil Data}

A large portion of the rotor disk of a full sized helicopter is usually exposed to Mach numbers where compressibility effects are significant and should be accounted for. However, the VII algorithm and XFOIL are both based on incompressible flow theories. In order to use the predicted airfoil properties, so called compressibility corrections can be applied to the incompressible airfoil surface pressure distribution. The most famous of these is known as the Prandtl-Glauert rule shown in Equation 3.7 [63].

$$
C_{p}=\frac{C_{p_{\text {inc }}}}{\sqrt{1-M_{\infty}^{2}}}
$$

The Prandtl-Glauert rule was derived assuming small perturbation velocities of thin airfoils and only provides adequate correction accuracy at low Mach numbers. It is, however, easily applied to scale the lift and moment coefficients due to the constant correction factor that is only dependent on the free-stream Mach number $M_{\infty}$.

Several modifications and improvements to the Prandtl-Glauert rule are available in the form of Laitone's rule and the Kármán-Tsien rule. Laitone's modification specifically considers the local Mach number distribution at the airfoil surface rather than the freestream Mach number. The Kármán-Tsien rule accounts for local velocity perturbations and again assumes small perturbations most applicable to thin profiles. Since both of these modifications are dependent on local airfoil properties, their application requires knowledge of the pressure distribution at the airfoil surface and can therefore not be used to operate on airfoil force coefficients directly. XFOIL allows specification of Mach number for the flow condition and applies the Kármán-Tsien correction on the airfoil pressure distribution prior to the force coefficient integrations [48].

Another compressibility correction in the sense of Prandtl-Glauert was published by 
Kaplan [64]. Kaplan's rule is based on a study of compressibility effects on elliptical cylinders and provides a way to account for the thickness of an airfoil as shown in Equation 3.8 [63]. Similar to the original Prandtl-Glauert derivation, this rule can be expressed based on free-stream flow conditions only and may therefore be applied to correct lift and moment coefficients directly.

$$
C_{p}=C_{p_{\text {inc }}}\left[\mu_{K}+\frac{t / c}{1+(t / c)}\left[\mu_{K}\left(\mu_{K}-1\right)+\frac{1}{4}(\gamma+1)\left(\mu_{K}^{2}-1\right)^{2}\right]\right],
$$

where $\mu_{K}$ is the Mach number effect as shown in Equation 3.9, $\gamma$ is the fluid's ratio of specific heats and ${ }^{t} / c$ is the thickness to chord ratio.

$$
\mu_{K}=\frac{1}{\sqrt{1-M_{\infty}^{2}}}
$$

Unfortunately these simple corrections do not account for compressibility effects on the drag coefficient. Drag will start to increase drastically once the Mach number exceeds the airfoil specific drag divergence Mach number, usually near $M=0.8$.

Both the Prandtl-Glauert and the Kaplan compressibility correction models are implemented in the simulation modules for axial and forward flight introduced in Sections 3.2 and 3.3. Either one of these models may be optionally selected to correct incompressible lift and moment coefficient data from the airfoil data look-up tables. Alternatively, if the two-dimensional airfoil data already accounts for Mach effect, the compressibility corrections may be deselected. 
CHAPTER 3. THE QOPTR SIMULATION MODULES

\subsection{Blade Element Momentum Theory Implementation for Hover and Axial Climb}

The basis of blade element momentum theory is described in Section 2.2.2.1. This section describes the BEMT implementation in the Qoptr hover module. The following list outlines the structure of a main analysis loop iteration and succeeding paragraphs elaborate on the steps in more detail.

1. The inflow ratio is calculated with optional loading corrections to account for tip and root losses.

2. Blade element angle of attack, Reynolds number and Mach number are determined and section aerodynamic lift, drag and moment coefficients are interpolated from airfoil parameter look-up tables.

3. Lift and drag coefficients are transformed into the rotor coordinate system.

4. Thrust and power coefficients are calculated and blade pitching moment coefficients are summed along the blade.

5. Several parameters are checked for convergence; collective pitch is adjusted to reach the desired thrust coefficient.

The inflow ratio distribution, defined in Equation 2.41, assumes a constant lift curve slope $C_{l_{\alpha}}$, small angles and a number of other simplifications as shown in Section 2.2.2.1. Since the airfoil properties in the simulation modules are provided by look-up tables and the lift curve slope is thus not assumed constant, an equation was derived to express the inflow in terms of the upward force produced at the blade section. 
Starting from the two thrust coefficient equations for an annulus of the disk (see Eqs. 2.31 and 2.36) and eliminating $\mathrm{d} C_{T} / \mathrm{d} r$ gives

$$
\frac{\sigma}{2} C_{F_{z}} r^{2}=4 \lambda\left(\lambda-\lambda_{c}\right) r
$$

when $U / \Omega R=r$ is assumed. Equation 3.10 can be expanded into a quadratic equation in terms of inflow ratio with the solution

$$
\lambda\left(r, \lambda_{c}\right)=\frac{\lambda_{c}}{2}+\sqrt{\left(\frac{\lambda_{c}}{2}\right)^{2}+\frac{\sigma C_{F_{z}} r}{8}}
$$

Simulation results obtained using Equation 3.11 were confirmed to be equivalent to results obtained from Equation 2.41, when $C_{F_{z}}$ was defined according to the assumptions of Section 2.2.2.1. Since the aerodynamic coefficients and hence $C_{F_{z}}$ are unknown at the start of the first iteration, Equation 2.41 is used with an assumed linear lift coefficient slope $C_{l_{\alpha}}=2 \pi$ to initialize the simulation.

Tip loss effects are optionally accounted for using Prandtl's loading correction function $F$ shown in Equation 2.17. This correction function is applied to the momentum theory expression in the preceding derivation of the inflow ratio leading to Equations 3.12 and 3.13 .

$$
\begin{gathered}
\frac{\sigma}{2} C_{F_{z}} r^{2}=4 F \lambda\left(\lambda-\lambda_{c}\right) r \\
\lambda\left(r, \lambda_{c}\right)=\frac{\lambda_{c}}{2}+\sqrt{\left(\frac{\lambda_{c}}{2}\right)^{2}+\frac{\sigma C_{F_{z}} r}{8 F}}
\end{gathered}
$$

If tip losses are ignored, the value of $\mathrm{F}$ becomes unity and Equations 3.12 and 3.13 reduce to Equations 3.10 and 3.11, respectively. Solving for the loading correction $F$ is complicated as it is dependent on inflow angle $\phi$, which is directly related to inflow 
ratio $\lambda$ by Equation 2.39. Inflow ratio $\lambda$ is in turn dependent on the loading correction $F$. An iterative procedure is therefore implemented, where $F$ is initialized at unity and Equations 3.13 and 2.17 are repeatedly solved for $\lambda$ and $F$ until $F$ converges to a constant value.

Leishman suggests that Prandtl's correction for the tip may also be modified and applied at the blade root. The two equations are shown in Equations 3.14 and 3.15 [13, pp. 141-145, 743].

$$
\begin{gathered}
F_{\text {tip }}=\frac{2}{\pi} \cos ^{-1}\left(e^{-\frac{N_{b}(1-r)}{2 r \phi}}\right) \\
F_{\text {root }}=\frac{2}{\pi} \cos ^{-1}\left(e^{-\frac{N_{b}\left(r-r_{0}\right)}{2 r \phi}}\right)
\end{gathered}
$$

$F_{\text {root }}$ is determined in the same iterative manner as described above for $F_{\text {tip }}$. The two corrections are multiplied at each section to produce a single overall correction function distribution.

Once the inflow ratio distribution is known, Equations 2.39 and 2.22 are used to determine the effective angle of attack $\alpha$ at the blade section and Reynolds number and Mach number are calculated using section velocity U from Equation 2.20. Given section angle of attack and chordwise Reynolds number the two-dimensional aerodynamic lift, drag and moment coefficients are interpolated for the specified airfoil at each section. Subsequent correction for lift and moment coefficient compressibility effects is optional as described in Section 3.1.3.

Using Equations 2.33 and 2.34, the lift and drag coefficients are transformed into components parallel and perpendicular to the rotor. Annulus thrust coefficient $\mathrm{d} C_{T}$ and power (or torque) coefficient $\mathrm{d} C_{P}$ are then calculated using Equations 2.31 and 2.32. Annulus power coefficient sub-components $\mathrm{d} C_{P_{i}}$ and $\mathrm{d} C_{P_{o}}$ accounting for induced (and 
climb) and profile power losses, respectively, are also determined by dividing Equation 2.32 into two parts:

$$
\begin{aligned}
& \mathrm{d} C_{P_{i}}=\frac{\sigma}{2} C_{l} \sin \phi \frac{U^{2}}{(\Omega R)^{2}} r \mathrm{~d} r \\
& \mathrm{~d} C_{P_{o}}=\frac{\sigma}{2} C_{d} \cos \phi \frac{U^{2}}{(\Omega R)^{2}} r \mathrm{~d} r
\end{aligned}
$$

Annulus thrust and power coefficients are then summed over the disk to provide the overall rotor thrust and power coefficients.

$$
\begin{aligned}
C_{T} & =\int_{r_{0}}^{1} \mathrm{~d} C_{T} \\
C_{P} & =\int_{r_{0}}^{1} \mathrm{~d} C_{P}
\end{aligned}
$$

Knowledge of the thrust and torque coefficients allows evaluation of the figure of merit as shown in Equation 2.12. Blade aerodynamic pitching moment is also calculated dimensionally at each blade section and integrated along the blade span to provide a value at the root as shown in Equation 3.20.

$$
\left.M_{r}\right|_{\text {Blade Root }}=\int_{r_{0}}^{1} \frac{1}{2} \rho U^{2} c^{2} C_{m} R \mathrm{~d} r
$$

The last step in the iteration is to determine whether the simulation has converged to a steady solution. This is done by evaluating the variation of three parameters: the inflow ratio distribution $\lambda(r)$ and the magnitudes of the thrust coefficient $C_{T}$ and figure of merit $F M$. The normalized root mean square difference (NRMSD) of the inflow ratio distribution is calculated with respect to the preceding iteration and compared against a limit specified as an input to the algorithm. The iteration goal of the hover module simulation can be set either to a specific thrust coefficient or to a certain collective pitch 
deflection. In the first case the error in thrust coefficient is calculated with respect to the specified input value and collective pitch is adjusted for the next iteration. If the solution for a specific collective level is desired, the difference in thrust coefficient is calculated with respect to the previous iteration.

Convergence of the figure of merit of the rotor configuration is also important as it is a main component of the optimization objective function in hover. Similar to the thrust coefficient treatment, the normalized difference between values of consecutive iterations is compared to a specified limit.

Collective adjustment is carried out using the procedure outlined in Reference [13, pp. 121, 130-131]. The thrust coefficient produced by a simple rotor assuming constant inflow through the disk and linear twist on the blades is defined as

$$
C_{T}=\frac{\sigma}{2} C_{l_{\alpha}}\left(\frac{\theta_{75}}{3}-\frac{\lambda}{2}\right)
$$

Solving Equation 3.21 for collective pitch $\theta_{75}$ and assuming that inflow is defined by the basic momentum theory value in hover (Equation 2.8) gives

$$
\theta_{75}=\frac{6 C_{T}}{\sigma C_{l_{\alpha}}}+\frac{3}{2} \sqrt{\frac{C_{T}}{2}}
$$

Therefore a correction to the collective pitch for iteration $(j+1)$ can be determined based on the difference of the current iteration's thrust coefficient and the desired value $C_{T_{(r e q)}}$ as follows.

$$
\theta_{75_{(j+1)}}=\theta_{75_{(j)}}+\frac{6\left(C_{T_{(r e q)}}-C_{T_{(j)}}\right)}{\sigma C_{l_{\alpha}}}+\frac{3 \sqrt{2}}{4}\left(\sqrt{C_{T_{(r e q)}}}-\sqrt{C_{T_{(j)}}}\right)
$$

This sequence of calculations is repeated until convergence is determined or the iteration limit has been reached. 


\subsubsection{Development Difficulties}

Recasting Equation 2.41 into Equation 3.13 resulted in a numerical instability in the convergence algorithm. This most likely occurred because the vertical force coefficient $C_{F_{z}}$, which is used in Equation 3.13, is calculated in the previous iteration and therefore prior to the collective pitch correction applied for the current iteration. The instability was alleviated by forming a sub-loop enclosing all calculations from the inflow ratio distribution to the calculation of the normal force coefficient and applying an underrelaxation scheme to the value of $C_{F_{z}}$. It was found that two iterations of the sub-loop per main loop iteration and relaxation factor $\omega_{C_{F_{z}}}=0.5$ resulted in efficient convergence in most cases.

A further problem is encountered due to the momentum theory assumption of positive (downward) inflow at the disk (see Section 2.2.1). When the rotor definition or a bad initial condition - results in a spanwise loading distribution which is weighted heavily toward a certain region on blade, off-loaded sections of the blade may predict negative values of normal force coefficient $C_{F_{z}}$. Application of loading correction functions $F_{\text {tip }}$ and $F_{\text {root }}$ may amplify this effect. Considering Equation 3.13 and assuming hover for simplicity shows that a negative value of $C_{F_{z}}$ would produce a complex value for the section inflow ratio. In order to prevent such non-physical results, all negative values of $C_{F_{z}}$ are set to zero and the algorithm is not allowed to exit in the current iteration. Using additional under-relaxation on the normal force coefficient and inflow ratio distribution, the algorithm attempts to converge to a solution that satisfies the assumptions of the theory. If no satisfactory solution is reached in the maximum number of iterations specified for the simulation run, a warning message is displayed to the screen. 


\subsubsection{Validation of the Qoptr Hover Module}

The output of the hover module was first verified against textbook solutions to problems presented in Reference [13]. During these tests various theoretical trends with respect to different radial twist and taper distributions, lift and drag coefficient definitions and the effects of the tip loss function were confirmed to perform as expected.

The module was then also used to simulate the scaled rotor tests specified in References [21], [22] and [23]. Resulting power coefficient and figure of merit variations with respect to thrust coefficient are included in Appendix B. The results for Reference [22] are also shown in Figure 3.4. In this study two rotors differing only by the airfoils used were evaluated. The baseline rotor airfoil was the NACA 0012 and the second rotor featured the US Army/NASA RC(4)-10, RC(3)-10 and the RC(3)-08 airfoils distributed over various regions of the blade span. Solidity, twist and taper
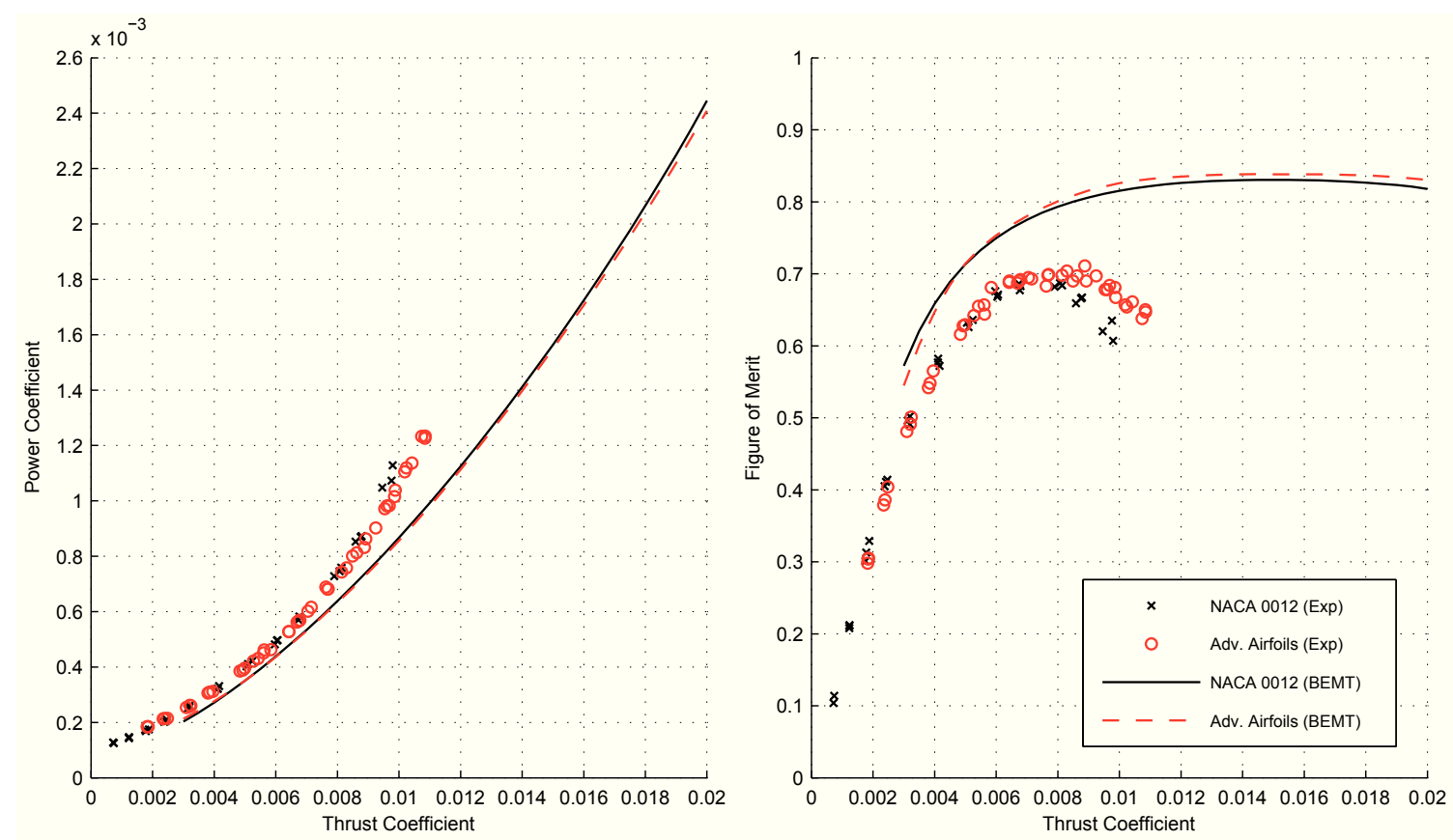

Figure 3.4: Power coefficient and figure of merit simulated using the hover module compared to experimental results from Ref. [22]. 
were the same for both rotors. Tests were performed at different tip speeds and the $172.8 \mathrm{~m} / \mathrm{s}(567 \mathrm{ft} / \mathrm{s})$ case is shown here.

It can be seen that the power coefficients returned by the hover module are low compared to the experimental results. It can also be seen that the difference from the experimental results increases with thrust coefficient. The approximate percent error varies from -8 to $-26 \%$ over the experimental thrust coefficient range. Considering the figure of merit plot in Figure 3.4 the effect of the underpredicted power coefficient is magnified. Not only is the magnitude of the figure of merit overpredicted by up to 35\% (see Figure 3.5), the peak of the predicted curve is also shifted significantly to higher thrust coefficients.

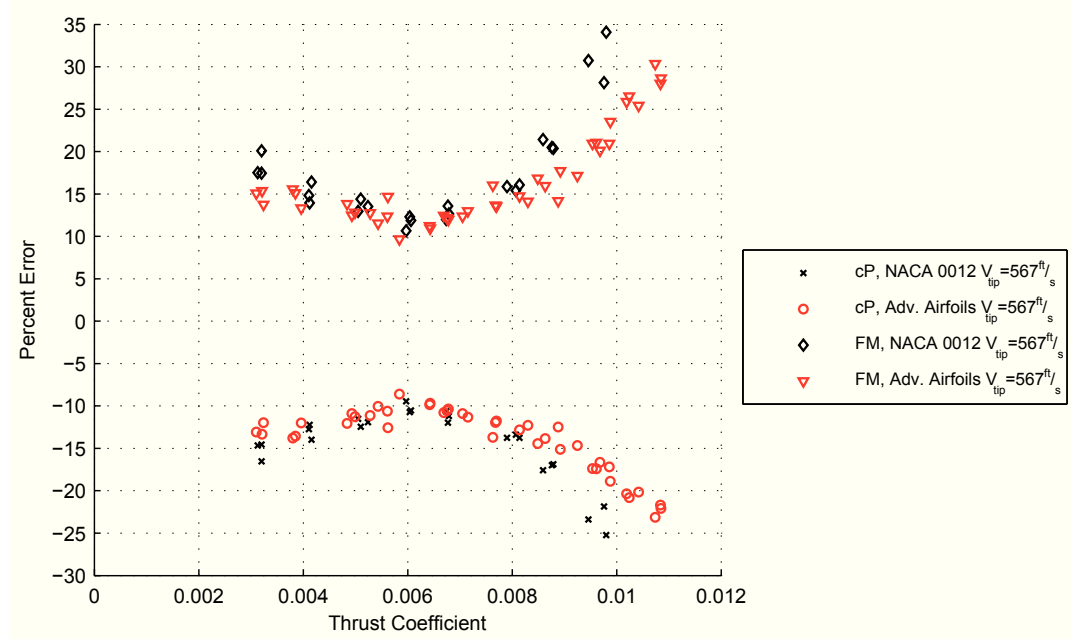

Figure 3.5: Error in simulated power coefficient and figure of merit predicted using the hover module.

All validation tests performed and presented in Appendix B show similar errors with respect to the experimental data from References [21], [22] and [23] as discussed here. Appendix B also includes results for full scale rotor tests presented in Reference [65]. Once again, similar trends with respect to power coefficient and figure of merit were observed. 
The authors of References [21], [22] and [23] compare their experimental results to several numerical methods including one based on blade element momentum theory. Similar trends of underpredicted power coefficients and resulting overprediction of figure of merit - including the shift of the figure of merit peak toward higher thrust coefficients - were published. Conclusions in Reference [21] suggest that this behaviour may be due to the fact that full scale airfoil data was used for the numerical assessments that did not represent the low Reynolds numbers encountered in the test. A subsequent analysis with airfoil data at a lower Reynolds number range - however, still not as low as the test condition - provided improved results for the blade element momentum theory based method.

In the present study, it is expected that the airfoil data produced using XFOIL may be responsible for a significant portion of the error in power coefficient. As discussed in Section 3.1.2, XFOIL tends to overpredict the maximum lift coefficient and underpredict drag coefficient at higher angles of attack. Using low Reynolds number experimental data for the NACA 0012 airfoil from Reference [66] the simulation for the baseline rotor already presented above was repeated. Due to turbulence levels in the wind tunnel used to produce the data presented in Reference [66] it is suggested that the test Reynolds numbers be corrected to an "effective" Reynolds number. Results for both simulations as well as those based on the XFOIL generated coefficients are shown in Figure 3.65.

The predicted power coefficient is increased to agree with the experimental results in the low thrust coefficient range. At higher thrust coefficients the power coefficient remains underpredicted, but shows better agreement with the experimental results than

\footnotetext{
${ }^{5}$ Application of the Reynolds number correction shifted the Reynolds number range of the data in Reference [66] to higher values, so that the new range did not completely encompass the range of Reynolds number simulated at the specified rotor condition. The coefficients for the lowest Reynolds number available were therefore applied to the affected rotor disk annuli.
} 

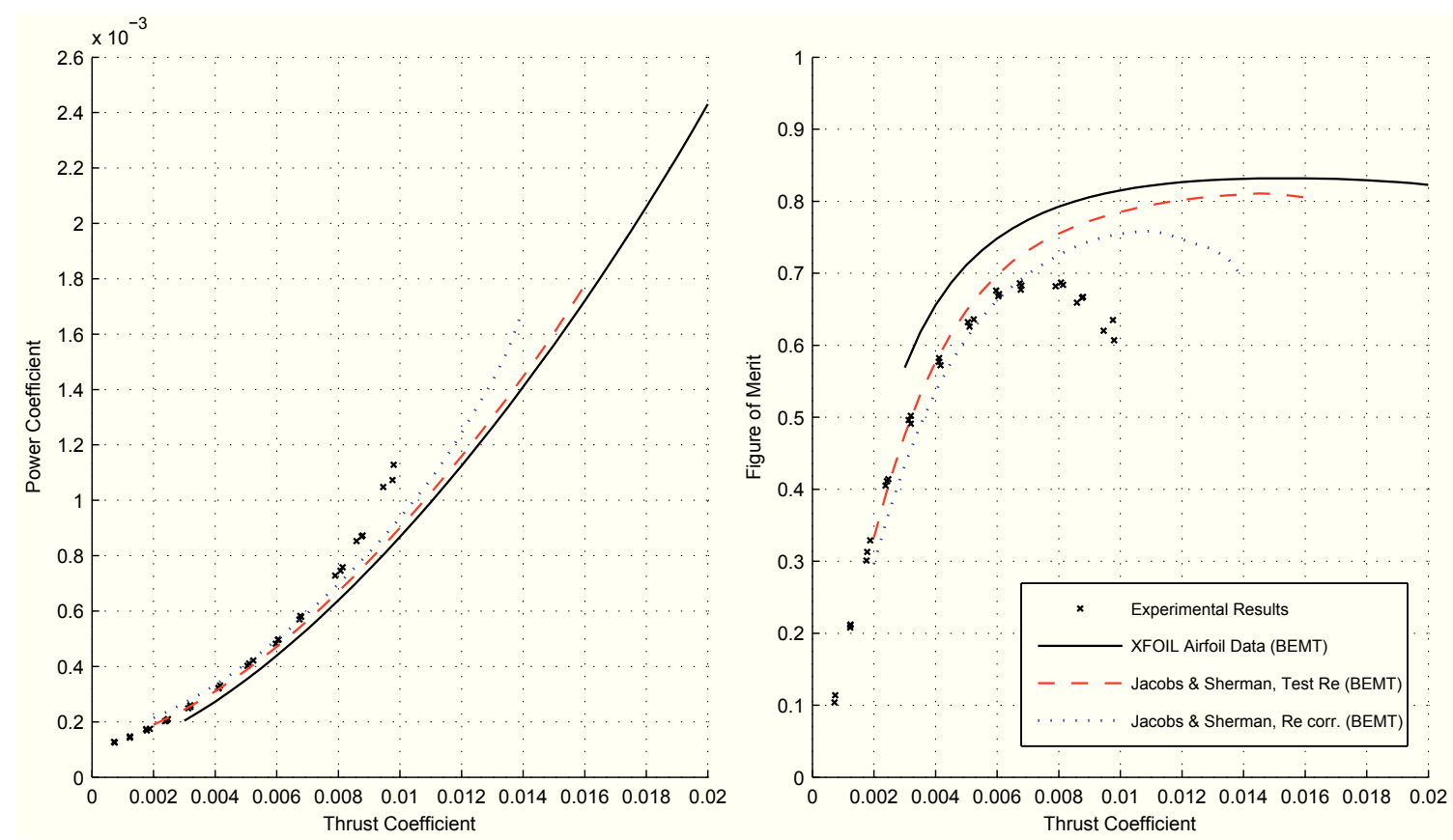

Figure 3.6: Comparison of simulated power coefficient and figure of merit using XFOIL and experimentally sourced [66] NACA 0012 airfoil data with respect to the experimental results from Ref. [22].

the curves produced using the XFOIL data. Considering the figure of merit fit, the simulation still overpredicts the maximum, but the peaks of the curves produced using the experimental low Reynolds number data from Reference [66] are reduced and shifted toward those seen in the experimental results.

In Figure 3.7 the percent error between the simulated data and the experimental data shows great improvement in the simulated results at low thrust coefficients with the use of the experimental airfoil coefficients that were not corrected for measurement turbulence levels ${ }^{6}$. Applying the correction actually caused an overprediction of power coefficient at low thrust coefficients.

At higher thrust coefficients, the simulated results continue to diverge from the experimental ones regardless of which airfoil dataset is used. This suggests that other

\footnotetext{
${ }^{6}$ See note 5
} 


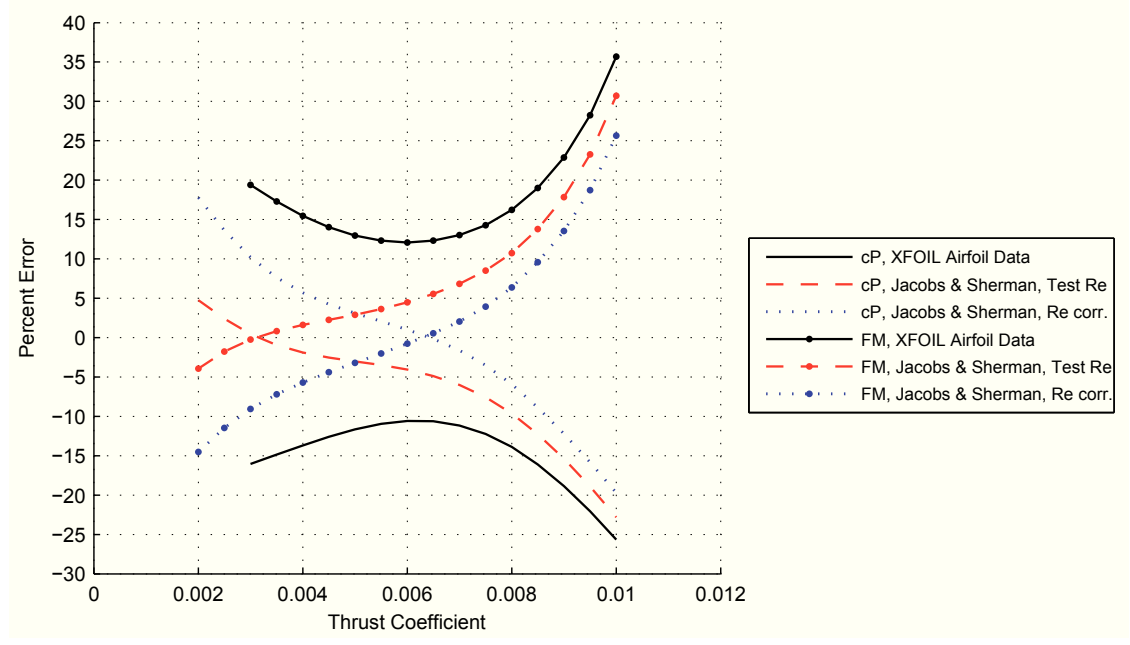

Figure 3.7: Error in simulated power coefficient and figure of merit predicted using XFOIL and experimentally sourced NACA 0012 airfoil data.

factors such as three-dimensional flow effects, elastic blade deformations, or flow interactions with the physical fuselage models used in the experiment may be at play.

\subsection{Blade Element Theory Implementation for Forward}

\section{Flight}

A general overview of blade element theory in forward flight is given in Section 2.2.2.2. In this section the BET implementation in the Qoptr forward flight module is described. A list of the general steps of the main simulation loop is provided, followed by a more detailed presentation of the underlying equations.

1. Blade dynamics and aerodynamics are determined iteratively moving azimuthally in the direction of rotation:

(a) Blade flapping and lead-lag velocities and deflection are determined.

(b) Local section flow velocities are calculated. 
(c) Blade element angle of attack, Reynolds number and Mach number are determined and section aerodynamic lift, drag and moment coefficients are interpolated from airfoil parameter look-up tables.

(d) Lift and drag coefficients are transformed into the reference coordinate system.

(e) Blade flapping and lead-lag accelerations are calculated from the blade equations of motion.

2. The one-per-rev flapping motion is characterized and the shaft angle of attack is updated.

3. Overall rotor force and moment coefficients are determined.

4. Several parameters are checked for convergence and collective and/or cyclic are adjusted to reach the desired thrust coefficient and rotor orientation.

5. If necessary, the induced inflow distribution is recalculated.

Similar to the BEMT implementation for hover, the rotor and the simulated flight condition is specified at the start of the simulation. The input parameters include the required thrust coefficient, the advance ratio $\mu_{x}$ and the disk angle of attack $\alpha_{T P P}$. In case performance parameters are to be simulated at specific control positions, an initial estimate for the thrust coefficient will be generated or may be supplied. These parameters are required to calculate the induced inflow based on forward flight momentum theory and various inflow models discussed in Sections 2.2.1.2 and 2.2.2.2, respectively.

In the forward flight module flap and lag deflections are defined as the physical angles of the flapping and lead-lag hinges. This is illustrated for a fully articulated rotor hub in Figure 3.8. The reference coordinate system for the blade motion derivation is 


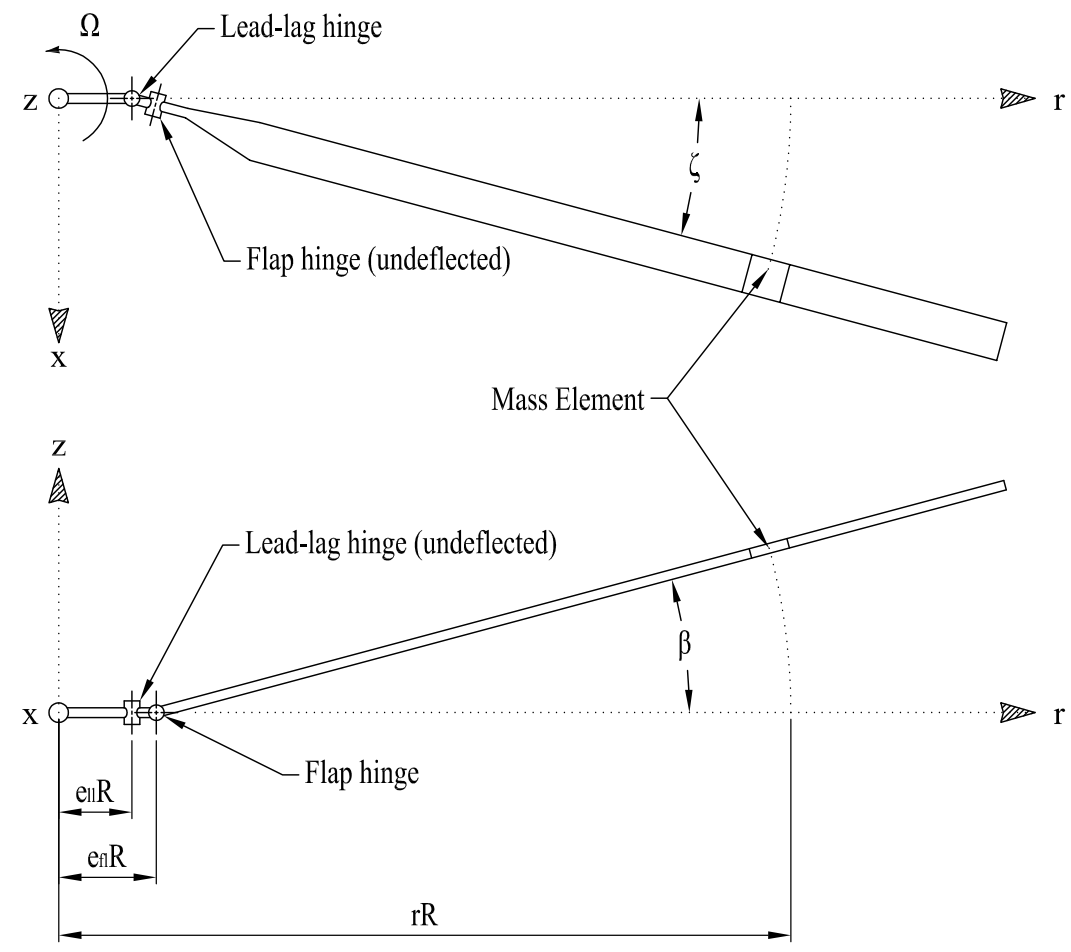

Figure 3.8: Fully articulated rotor hub layout in the hub-based rotating reference frame.

fixed to the rotor hub and is defined by the hub plane positioned perpendicular to the rotor shaft, and therefore fixed to the vehicle. In contrast, the orientation of the tip path plane is related to the vehicle attitude by the one-per-rev flapping motion. Flap and lead-lag derivatives are denoted $\beta^{*}, \beta^{* *}, \zeta^{*}$ and $\zeta^{* *}$ and represent the respective quantities normalized by rotorspeed $\Omega$ as shown in Equations 3.24 and 3.25.

$$
\begin{array}{ll}
\beta^{*}=\frac{\dot{\beta}}{\Omega} & \beta^{* *}=\frac{\ddot{\beta}}{\Omega^{2}} \\
\zeta^{*}=\frac{\dot{\zeta}}{\Omega} & \zeta^{* *}=\frac{\ddot{\zeta}}{\Omega^{2}}
\end{array}
$$

Blade flapping and lead-lag velocities and accelerations as well as lag deflections are initialized at zero. The initial blade flapping deflection is set to the precone angle for teetering rotors or to zero for fully articulated rotors. 
An explicit second order Adams-Bashforth scheme is utilized to integrate blade motion velocities and deflections from information at the two preceding azimuth positions. Using the normalized forms of the blade motion derivatives the temporal integration is performed per increment azimuth as shown in Equation 3.26.

$$
\begin{aligned}
\dot{\beta}_{n} & =\dot{\beta}_{(n-1)}+\left(\frac{3}{2} \ddot{\beta}_{(n-1)}-\frac{1}{2} \ddot{\beta}_{(n-2)}\right) \mathrm{d} t \\
\Omega \beta_{n}^{*} & =\Omega \beta_{(n-1)}^{*}+\left(\frac{3}{2} \Omega^{2} \beta_{(n-1)}^{* *}-\frac{1}{2} \Omega^{2} \beta_{(n-2)}^{* *}\right) \frac{\mathrm{d} \psi}{\Omega} \\
\beta_{n}^{*} & =\beta_{(n-1)}^{*}+\left(\frac{3}{2} \beta_{(n-1)}^{* *}-\frac{1}{2} \beta_{(n-2)}^{* *}\right) \mathrm{d} \psi
\end{aligned}
$$

Flap deflection and lead-lag velocity and deflection are determined similarly.

$$
\begin{aligned}
& \beta_{n}=\beta_{(n-1)}+\left(\frac{3}{2} \beta_{(n-1)}^{*}-\frac{1}{2} \beta_{(n-2)}^{*}\right) \mathrm{d} \psi \\
& \zeta_{n}^{*}=\zeta_{(n-1)}^{*}+\left(\frac{3}{2} \zeta_{(n-1)}^{* *}-\frac{1}{2} \zeta_{(n-2)}^{* *}\right) \mathrm{d} \psi \\
& \zeta_{n}=\zeta_{(n-1)}+\left(\frac{3}{2} \zeta_{(n-1)}^{*}-\frac{1}{2} \zeta_{(n-2)}^{*}\right) \mathrm{d} \psi
\end{aligned}
$$

Knowledge of the flap and lag positions and velocities at the current azimuth position allows the calculation of velocity at the blade sections. General equations were introduced in Section 2.2.2.2 (Eqs. 2.42 and 2.43). Adding flapping hinge location $e_{f l}$ as well as lag motion about $e_{l l}$ and non-dimensionalizing gives Equations 3.30 and 3.31. Coupled flap-lag effects on section velocity are neglected.

$$
\begin{gathered}
\frac{U_{P}}{\Omega R}=\mu_{z_{\text {ref }}}+\lambda_{i}+\left(r-e_{f l}\right) \beta^{*}+\mu_{x_{\text {ref }}} \beta \cos \psi \\
\frac{U_{T}}{\Omega R}=r+\mu_{x_{r e f}} \sin \psi-\left(r-e_{l l}\right) \zeta^{*}
\end{gathered}
$$


The advance ratios $\mu_{z_{\text {ref }}}$ and $\mu_{x_{\text {ref }}}$ are values perpendicular and parallel to the hubplane and are calculated using the shaft angle of attack $\alpha_{\text {shaft }}$ as shown in Equations 3.32 to 3.34 .

$$
\begin{aligned}
& \mu_{z_{\text {ref }}}=\frac{V_{\infty}}{\Omega R} \sin \alpha_{\text {shaft }} \\
& \mu_{x_{\text {ref }}}=\frac{V_{\infty}}{\Omega R} \cos \alpha_{\text {shaft }} \\
& \alpha_{\text {shaft }}=\alpha_{T P P}-\beta_{\text {long }},
\end{aligned}
$$

where $\beta_{\text {long }}$ is the longitudinal disk tilt due to flapping.

The perpendicular and tangential velocity components are summed vectorially to give the resultant velocity and allow determination of the inflow angle $\phi$ and the effective angle of attack $\alpha$ at the blade section as shown in Equations 2.20 to 2.22. To account for extreme flow incidence angles seen in the vicinity of the reverse flow region the range of the inverse tangent function was expanded as shown to return a value between $-\pi$ and $\pi$.

$$
\phi=\tan ^{-1}\left(\frac{U_{P}}{U_{T}}\right)+\operatorname{sign}\left(U_{P}\right) \frac{-\operatorname{sign}\left(U_{T}\right)+1}{2} \pi
$$

After calculating the section Reynolds numbers and Mach numbers, airfoil section lift, drag and moment coefficients are interpolated from lookup table databases. Incompressible lift and moment coefficients may be subsequently corrected for compressibility effects using the methods presented in Section 3.1.3.

The aerodynamic blade force coefficients are then transformed from the blade section into a rotating reference frame as shown in Equation 3.36, where the rotation 
matrix represents intrinsic rotations through the flapping and lead-lag hinges ${ }^{7}$. The rotating reference frame shares the z-axis with the fixed hub reference frame. Coordinate $\mathrm{x}$ is in the tangential direction opposite to the rotation of the rotor and $r$ is radially outward.

$$
\left\{\begin{array}{c}
C_{F_{x}} \\
C_{F_{r}} \\
C_{F_{z}}
\end{array}\right\}=\left[\begin{array}{ccc}
\cos \zeta & \cos \beta \sin \zeta & -\sin \beta \sin \zeta \\
-\sin \zeta & \cos \beta \cos \zeta & -\sin \beta \cos \zeta \\
0 & \sin \beta & \cos \beta
\end{array}\right]\left\{\begin{array}{c}
C_{l} \sin \phi+C_{d} \cos \phi \\
0 \\
\left(C_{l} \cos \phi-C_{d} \sin \phi\right) F_{\text {tip }}
\end{array}\right\}
$$

Reference [30, pp. 133-134] suggests that tip losses in a forward flight BET implementation can be accounted for by assuming that blade sections beyond a certain radius near the blade tip do not produce any thrust but continue to contribute to rotor torque. In order to prevent an abrupt discontinuity in the thrust distribution and remove the necessity of a rather ambiguous cut-off radius input an approach based on the aforementioned Prandtl tip loss correction function (Eq. 2.17) was implemented in the forward flight module. Since the varying dynamic environment that the rotor is exposed to in forward flight differs greatly from the quasi-steady operating and loading conditions seen on propellers for which Prandtl originally developed his tip loss function, it should be noted that the accuracy of the implementation is unconfirmed and likely poor. The changing operating conditions also create a challenge to the implementation of the tip loss function as the equation is only mathematically viable if the exponent $\frac{-N_{b}(1-r)}{2 r \phi}$ is negative. Considering the parameters involved, this requires that the inflow angle $\phi$ be positive at all times, which is not a practical requirement in forward flight. It was found that an azimuthally averaged inflow angle distribution could be used to allow

\footnotetext{
${ }^{7}$ Equation 3.36 only accounts for the reorientation of the direction of the blade element forces. The displacement of the deflected blade sections with respect to their undeflected positions in the rotating reference frame is assumed to be small and therefore neglected.
} 
the expression to be evaluated reliably. The vertical blade section force component $C_{F_{z}}$ is multiplied by the resulting correction factor distribution.

Knowing the aerodynamic forces at each blade section, the blade flapping and leadlag equations of motion are used to determine the resulting flap and lead-lag accelerations. Rigid blade motion is implemented in the forward flight module. This allows the rotor blade to flap or lag about the respective hinge locations, but restricts any elastic deformation like bending or twisting. Johnson states that rigid blade motion is sufficient to determine rotor performance characteristics [30, p. 168]. Using the rigid blade assumption and given rotor geometry parameters such as hinge locations, precone angle $\beta_{p}$ and undersling distance $e_{u s}$, the position of any section of the blade can be defined relative to the shaft by its radial location along the span and the blade flap and lag deflections, where $\beta$ and $\zeta$ are the time varying degrees of freedom in the dynamic system. The equations of motion for fully articulated and teetering rotor head configurations were derived and the full derivations are presented in Appendix C. Two teetering rotor configurations were implemented, one represents a traditional teetering hub on a full scale helicopter which does not include a lead-lag hinge, i.e. stiff in-plane, while the other includes lead-lag hinges to represent common $\mathrm{r} / \mathrm{c}$ helicopter teetering hubs which allow lead-lag motion about the blade attachment fastener. The second type of teetering rotor configuration is shown in Figure 3.9. The final forms of the equations of motion are presented here. 


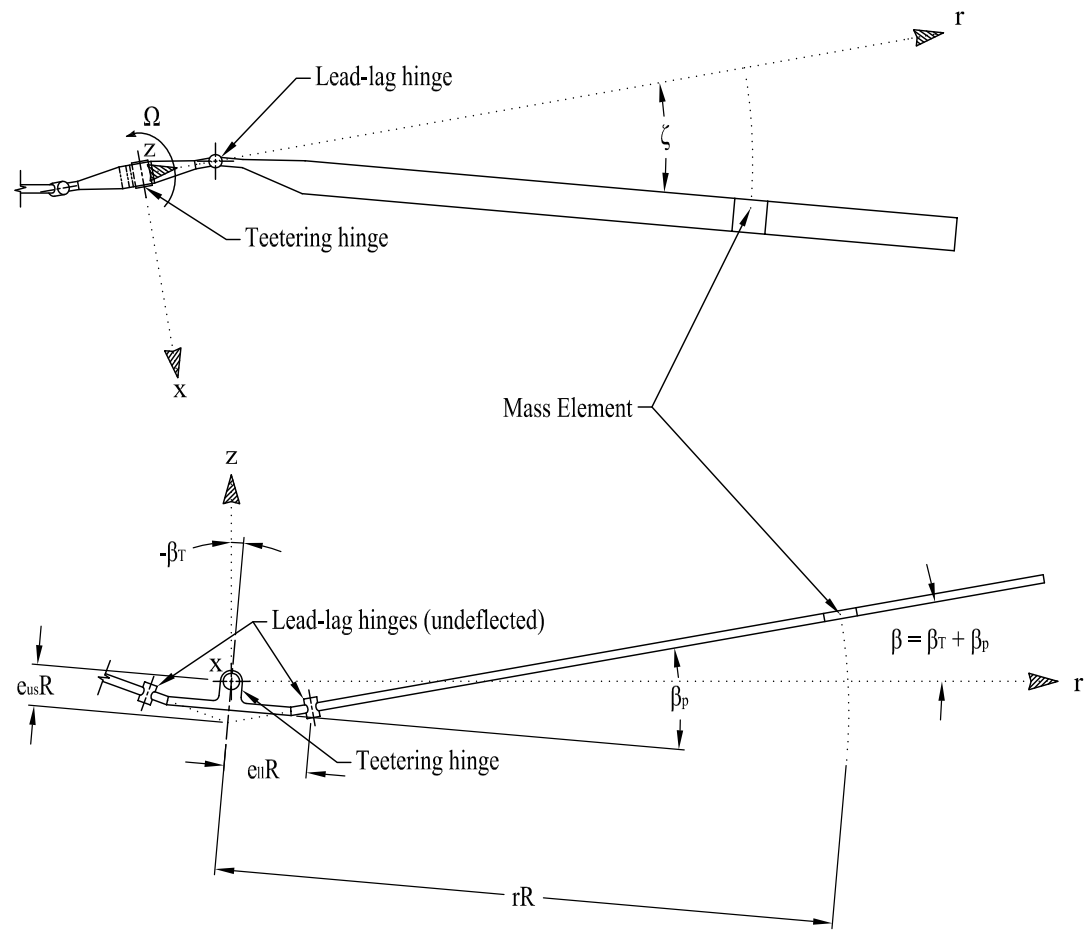

Figure 3.9: Soft in-plane teetering rotor hub layout in the hub-based rotating reference frame.

Fully articulated rotor:

$$
\begin{gathered}
\beta^{* *}=-v_{\beta}^{2} \beta+2 \frac{I_{C o r}}{I_{\beta}} \beta \zeta^{*}+\frac{K_{\beta}}{I_{\beta} \Omega^{2}} \beta_{p}+\frac{R}{I_{\beta} \Omega^{2}} \int_{e_{f l}}^{1}\left(r-e_{f l}\right)\left(F_{z}-F_{r} \beta\right) \mathrm{d} r \\
\zeta^{* *}=-v_{\zeta}^{2} \zeta-2 \frac{I_{C o r}}{I_{\zeta}} \beta \beta^{*}-\frac{C_{\zeta}}{I_{\zeta} \Omega} \zeta^{*}+\frac{R}{I_{\zeta} \Omega^{2}} \int_{e_{l l}}^{1}\left(r-e_{l l}\right)\left(F_{x}-F_{r} \zeta\right) \mathrm{d} r
\end{gathered}
$$

Teetering rotor (soft in-plane):

$$
\begin{aligned}
\beta_{T}^{* *}=-v_{\beta_{T}}^{2} \beta_{T}+2 \frac{I_{C o r_{1}}}{I_{\beta_{T}}} \beta_{T}\left(\zeta_{\psi}^{*}+\zeta_{\psi+\pi}^{*}\right)+2 \frac{I_{C o r_{2}}}{I_{\beta_{T}}}\left(\zeta_{\psi}^{*}-\zeta_{\psi+\pi}^{*}\right) \\
+\frac{R}{I_{\beta_{T}} \Omega^{2}}\left[\int_{0}^{1} F_{z_{\psi}}\left(r-\left(\beta_{p} r-e_{u s}\right) \beta_{T}\right) \mathrm{d} r-\int_{0}^{1} F_{z_{\psi+\pi}}\left(r+\left(\beta_{p} r-e_{u s}\right) \beta_{T}\right) \mathrm{d} r\right. \\
\left.\quad-\int_{0}^{1} F_{r_{\psi}}\left(\left(\beta_{T}+\beta_{p}\right) r-e_{u s}\right) \mathrm{d} r+\int_{0}^{1} F_{r_{\psi+\pi}}\left(\left(\beta_{T}-\beta_{p}\right) r+e_{u s}\right) \mathrm{d} r\right]
\end{aligned}
$$




$$
\zeta^{* *}=-v_{\zeta}^{2} \zeta-2 \frac{I_{C_{0 r_{1}} \beta_{T}+I_{C o r_{2}}}}{I_{\zeta}} \beta_{T}^{*}-\frac{C_{\zeta}}{I_{\zeta} \Omega} \zeta^{*}+\frac{R}{I_{\zeta} \Omega^{2}} \int_{e_{l l}}^{1}\left(r-e_{l l}\right)\left(F_{x}-F_{r} \zeta\right) \mathrm{d} r
$$

Teetering rotor (fixed in-plane):

$$
\begin{gathered}
\beta_{T}^{* *}=-v_{\beta_{T}}^{2} \beta_{T}+\frac{R}{I_{\beta_{T}} \Omega^{2}}\left[\int_{0}^{1} F_{z_{\psi}}\left(r-\left(\beta_{p} r-e_{u s}\right) \beta_{T}\right) \mathrm{d} r-\int_{0}^{1} F_{z_{\psi+\pi}}\left(r+\left(\beta_{p} r-e_{u s}\right) \beta_{T}\right) \mathrm{d} r\right. \\
\left.-\int_{0}^{1} F_{r_{\psi}}\left(\left(\beta_{T}+\beta_{p}\right) r-e_{u s}\right) \mathrm{d} r+\int_{0}^{1} F_{r_{\psi+\pi}}\left(\left(\beta_{T}-\beta_{p}\right) r+e_{u s}\right) \mathrm{d} r\right]
\end{gathered}
$$

Equations for the individual parameters in the equations of motion are shown in Appendix C. $I_{\beta}, I_{\zeta}$ and $I_{\beta_{T}}$ represent the flapping, lead-lag and teetering moments of inertia. $I_{\text {Cor }}$ accounts for the relative placement of hinges on the Coriolis acceleration terms. The non-dimensional natural flapping, lead-lag and teetering frequencies are denoted $v_{\beta}, v_{\zeta}$ and $v_{\beta_{T}}$, respectively. Constants $K_{\beta}$ and $C_{\zeta}$ represent optional flapping or teetering hinge spring stiffness and lead-lag damping coefficients in units of torque per angular displacement and torque per angular velocity, respectively ${ }^{8}$.

Force distributions $F_{x}, F_{r}$ and $F_{z}$ are the aerodynamic force components per unit blade span in the rotational reference frame as defined in Equation 3.42 to 3.44. In the teetering degree of freedom equations subscripts are used on the force terms to account for forces being applied at the current azimuth as well as those acting on the opposite blade, i.e. at azimuth $\psi+\pi$.

$$
\begin{aligned}
& F_{x}=\frac{1}{2} \rho U^{2} c C_{F_{x}} \\
& F_{r}=\frac{1}{2} \rho U^{2} c C_{F_{r}} \\
& F_{z}=\frac{1}{2} \rho U^{2} c C_{F_{z}}
\end{aligned}
$$

\footnotetext{
${ }^{8}$ Specifying zero lead-lag hinge damping for forward flight module simulations leads to convergence challenges and may cause the simulation to "blow up". It is suggested that at least a small amount of damping coefficient be specified to find a solution. This amount may subsequently be reduced, if necessary.
} 
The calculations presented up to this point are repeated at the next azimuth location until properties over the complete rotor disk have been determined.

Once calculations have been performed at each azimuth location the one-per-rev longitudinal and lateral disk deflections $\beta_{\text {long }}$ and $\beta_{\text {lat }}$ are determined from the azimuthal flapping angle variation using a least squared approach on the relation shown in Equation 3.45. The lag deflection is neglected in this process.

$$
\left(1-e_{f l}\right) \beta(\psi) \approx \beta_{0}+\beta_{\text {long }} \cos \psi+\beta_{\text {lat }} \sin \psi(+\ldots \text { higher order terms })
$$

where $\beta$ is the flapping hinge deflection and $\beta_{0}$ is the coning angle. The longitudinal disk deflection angle is then used in conjunction with Equation 3.34 to update the shaft angle of attack.

Knowledge of the aerodynamic force distribution across the rotor disk also allows these force components to be combined to total rotor force and moment coefficients relative to the vehicle fixed hub reference frame. Equations 3.46 to 3.51 are used to estimate these coefficients. Dynamic rotor effects and hinge deflections are neglected. Rotor forces include thrust $T$, the rotor drag force $H$ and the rotor side force $Y$.

$$
\begin{gathered}
C_{T_{r e f}}=\frac{1}{\rho(\Omega R)^{2} \pi R^{2}} \frac{N_{b}}{2 \pi} \int_{0}^{2 \pi} \int_{0}^{1} F_{z} R \mathrm{~d} r \mathrm{~d} \psi \\
=\frac{N_{b}}{2 \rho \pi^{2}(\Omega R)^{2} R^{2}} \int_{0}^{2 \pi} \int_{0}^{1} \frac{1}{2} \rho U_{n d}^{2}(\Omega R)^{2} R c C_{F_{z}} \mathrm{~d} r \mathrm{~d} \psi \\
C_{T_{r e f}}=\frac{1}{4 \pi} \int_{0}^{2 \pi} \int_{0}^{1} U_{n d}^{2} \sigma C_{F_{z}} \mathrm{~d} r \mathrm{~d} \psi \\
C_{H_{r e f}}=\frac{1}{4 \pi} \int_{0}^{2 \pi}\left[\int_{0}^{1} U_{n d}^{2} \sigma C_{F_{x}} \mathrm{~d} r \sin \psi+\int_{0}^{1} U_{n d}^{2} \sigma C_{F_{r}} \mathrm{~d} r \cos \psi\right] \mathrm{d} \psi \\
C_{Y_{r e f}}=\frac{1}{4 \pi} \int_{0}^{2 \pi}\left[\int_{0}^{1}-U_{n d}^{2} \sigma C_{F_{x}} \mathrm{~d} r \cos \psi+\int_{0}^{1} U_{n d}^{2} \sigma C_{F_{r}} \mathrm{~d} r \sin \psi\right] \mathrm{d} \psi
\end{gathered}
$$


Total rotor moments are rotor torque $Q^{9}$, rotor rolling moment $M_{x}$ and rotor pitching moment $M_{y}$.

$$
\begin{aligned}
C_{Q_{\text {ref }}}= & -\frac{1}{\rho(\Omega R)^{2}\left(\pi R^{2}\right) R} \frac{N_{b}}{2 \pi} \int_{0}^{2 \pi} \int_{0}^{1} R^{2} F_{x} \mathrm{~d} r \mathrm{~d} \psi \\
= & -\frac{N_{b}}{2 \rho \pi^{2}(\Omega R)^{2} R^{3}} \int_{0}^{2 \pi} \int_{0}^{1} \frac{1}{2} \rho U_{n d}^{2}(\Omega R)^{2} R^{2} c C_{F_{x}} r \mathrm{~d} r \mathrm{~d} \psi \\
C_{Q_{\text {ref }}}= & -\frac{1}{4 \pi} \int_{0}^{2 \pi} \int_{0}^{1} U_{n d}^{2} \sigma C_{F_{x}} r \mathrm{~d} r \mathrm{~d} \psi \\
C_{M_{x_{r e f}}} & \frac{1}{4 \pi} \int_{0}^{2 \pi} \int_{0}^{1} U_{n d}^{2} \sigma C_{F_{z}} r \mathrm{~d} r \sin \psi \mathrm{d} \psi \\
& C_{M_{y_{r e f}}}=\frac{1}{4 \pi} \int_{0}^{2 \pi} \int_{0}^{1} U_{n d}^{2} \sigma C_{F_{z}} r \mathrm{~d} r \cos \psi \mathrm{d} \psi
\end{aligned}
$$

Blade section thrust and power coefficient distributions are also calculated for each blade section and azimuth. When the Mangler and Squire inflow model is selected the Type I and Type III superposition coefficients are determined using a least square approach on Equation 3.52, where $\mathrm{d} C_{T_{a v e}}(r)$ is the azimuthally averaged section thrust coefficient distribution and $C_{a r b}$ is an arbitrary constant. The values of $w_{1}$ and $w_{3}$ are limited to lie between 0 and 1 .

$$
\mathrm{d} C_{T_{\text {ave }}}(r)=C_{\text {arb }}\left(w_{1} \frac{3}{4} \sqrt{1-r^{2}}+w_{3} \frac{15}{8} r^{2} \sqrt{1-r^{2}}\right)
$$

Using the latest azimuthally averaged inflow angle distribution the tip loss function $F_{\text {tip }}$ is updated.

At the end of the simulation loop thrust coefficient and blade motion variations are checked for convergence within limits set as inputs. The conditions on thrust coefficient

\footnotetext{
${ }^{9}$ Using the hub based reference frame where the z-axis is oriented positive up requires a sign change on the torque coefficient $C_{Q}$ relative to the power coefficient $C_{P}$.
} 
are equivalent to those described for the hover module. For flapping and lead-lag motions the RMS difference of the respective acceleration profiles relative to the previous iteration are used to determine convergence.

Depending on the simulation goal - specified thrust coefficient or specified collective pitch - parameters are then modified to attempt to converge on the desired solution. If a desired value of thrust coefficient is specified collective pitch is adjusted as described for the hover module in Section 3.2 and the effect of the updated shaft angle of attack is taken into account. Alternatively, if a desired collective pitch value is specified, thrust coefficient convergence is tested with respect to the value from the previous iteration and the inflow distribution is recalculated based on the most recent thrust coefficient.

The forward flight module also includes two trim routines. A wind-tunnel trim routine can be activated to impose cyclic pitch adjustments to remove the one-perrev flapping motion in the hub reference frame. The second trim routine is a simple implementation that attempts to achieve full vehicle trim. In this case additional vehicle parameters must be specified to define the vehicle centre of gravity, the main and tail rotor moment arms, fuselage drag, etc.

This concludes the calculations performed in the simulation loop. The steps are repeated until convergence is achieved or the iteration limit is reached.

After exiting the simulation loop the overall rotor forces and moments as well as power are calculated from their respective coefficients as shown in Equations 3.53 to 3.59

$$
\begin{aligned}
& T_{r e f}=\rho(\Omega R)^{2}\left(\pi R^{2}\right) C_{T_{\text {ref }}} \\
& H_{\text {ref }}=\rho(\Omega R)^{2}\left(\pi R^{2}\right) C_{H_{r e f}} \\
& Y_{\text {ref }}=\rho(\Omega R)^{2}\left(\pi R^{2}\right) C_{Y_{\text {ref }}}
\end{aligned}
$$




$$
\begin{gathered}
Q_{r e f}=\rho(\Omega R)^{2}\left(\pi R^{2}\right) R C_{Q_{r e f}} \\
M_{x_{r e f}}=\rho(\Omega R)^{2}\left(\pi R^{2}\right) R C_{M_{x_{r e f}}} \\
M_{y_{r e f}}=\rho(\Omega R)^{2}\left(\pi R^{2}\right) R C_{M_{y_{r e f}}} \\
P=\rho(\Omega R)^{3}\left(\pi R^{2}\right) C_{P}
\end{gathered}
$$

For reference, the forces and moments are transformed from the hub reference frame into the tip path plane coordinate system by intrinsic rotations through the longitudinal and lateral rotor disk deflections using the rotation matrix shown in Equation 3.6010.

$$
\left\{\begin{array}{c}
x_{T P P} \\
y_{T P P} \\
z_{T P P}
\end{array}\right\}=\left[\begin{array}{ccc}
\cos \beta_{\text {long }} & 0 & \sin \beta_{\text {long }} \\
-\sin \beta_{\text {lat }} \sin \beta_{\text {long }} & \cos \beta_{\text {lat }} & \sin \beta_{\text {lat }} \cos \beta_{\text {long }} \\
-\cos \beta_{\text {lat }} \sin \beta_{\text {long }} & -\sin \beta_{\text {lat }} & \cos \beta_{\text {lat }} \cos \beta_{\text {long }}
\end{array}\right]\left\{\begin{array}{c}
x_{\text {ref }} \\
y_{r e f} \\
z_{\text {ref }}
\end{array}\right\}
$$

The total blade aerodynamic pitching moment is also calculated using Equation 3.20 at each azimuth location. No attempt is made to account for dynamic pitching moment effects.

\subsubsection{Validation of the Qoptr Forward Flight Module}

The Qoptr forward flight module was used to simulate the conditions presented by Harris in Reference [67]. This paper included measurements of rotor deflections and rotor force and moment coefficients for a wind-tunnel test series conducted using a scaled $\mathrm{CH}-47 \mathrm{C}$ rotor. The $1.66 \mathrm{~m}$ diameter rotor featured a four-bladed fully articulated rotor

\footnotetext{
${ }^{10}$ The disk deflection angles $\beta_{\text {lat }}$ and $\beta_{\text {long }}$ represent the rotor tip path plane orientation with respect to the stationary hub reference frame as determined in Equation 3.45.
} 
hub. The Boeing-Vertol V23010-1.58 airfoil was used along the span of the blade and XFOIL was used to generate the required low Reynolds number airfoil coefficient data for the simulation. Further rotor parameters are summarized in Table D.1 of Appendix D.

The data published in Reference [67] consists of three parameter sweeps. The first sweep presents data at advance ratios from 0 to 0.24 at a constant thrust coefficient solidity ratio of 0.08 . As the paper focuses on lateral flapping effects at low advance ratios the remaining parameter sweeps - one with respect to collective and one with respect to shaft angle of attack - were conducted at an advance ratio of 0.08 , the condition at which maximum lateral flapping effects were observed. In this section the performance of the forward flight module will be compared against the advance ratio sweep. Appendix D contains additional comparisons to the other two sweeps.

Results obtained using different inflow models are shown in Figure 3.10. Input parameters to the simulation were the disk angle of attack $\alpha_{T P P}$, the advance ratio $\mu_{x}$ and the thrust coefficient $C_{T}$.

Overall, the trends observed in the experimental study were reproduced by the simulation. The most distinguished differences between the simulated and experimental data seemed to be relatively constant offsets of approximately $3^{\circ}$ in collective pitch and 0.0014 in power coefficient to solidity ratio across the complete range of advance ratios. An increasing error in coning angle and rotor drag force was also observed with increasing advance ratio.

The selection of inflow model had significant effects on the simulation results. Assuming constant inflow resulted in a severe underrepresentation of lateral effects in flapping magnitude, as well as rotor side force and rolling moment. The Drees inflow model captured the longitudinal trends best, while the Pitt \& Peters model proved most reliable in reproducing lateral effects. As noted in Section 2.2.2.2, the implemented Pitt 

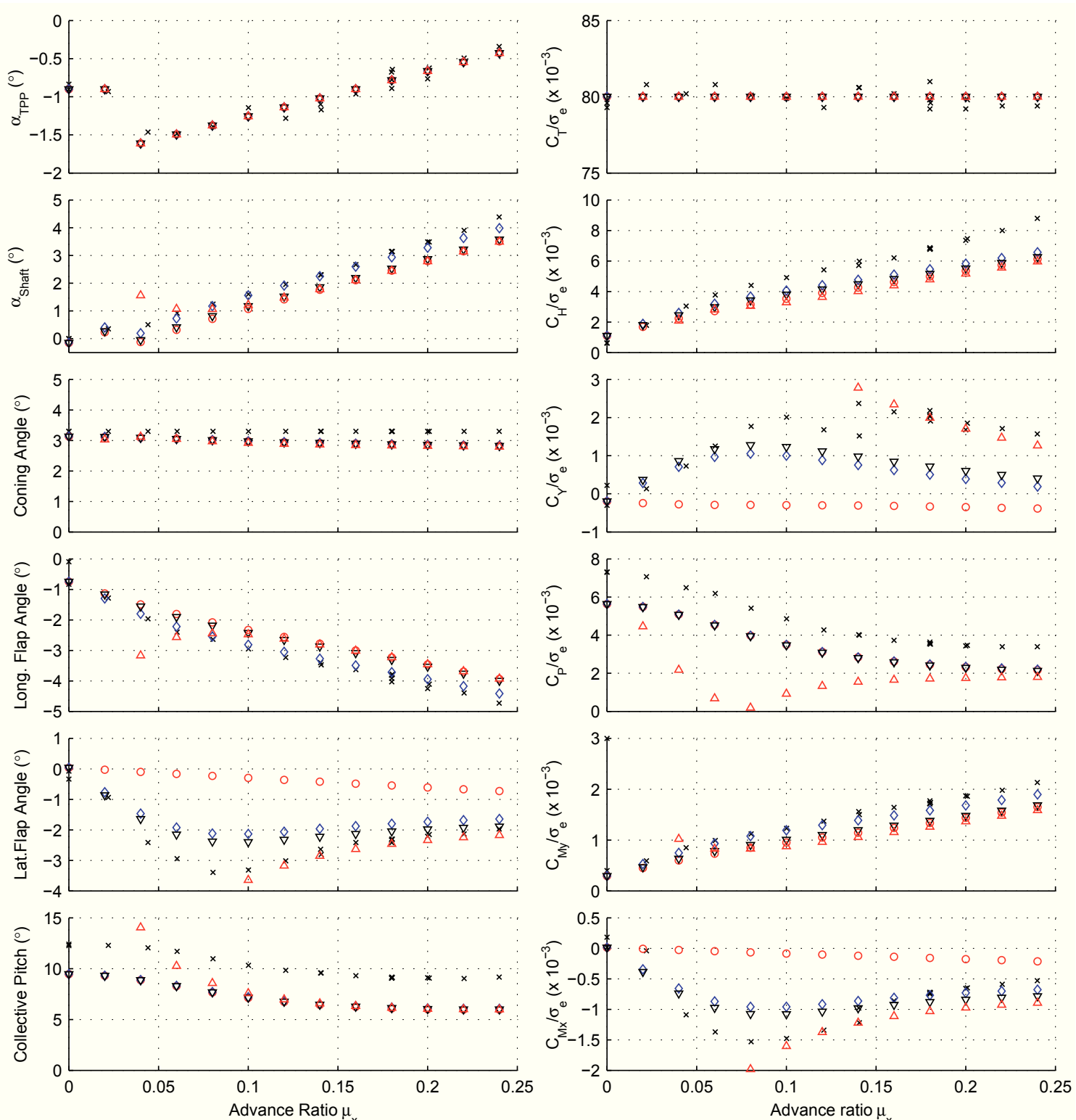

$$
\begin{array}{lllllllllll}
\hline \times & \text { Experimental } & \circ & \text { Constant } & \diamond & \text { Drees } & \nabla & \text { Pitt\&Peters } & \Delta & \text { Mangler\&Squire } \\
\hline
\end{array}
$$

Figure 3.10: Comparison of forward flight module simulated results to experimental data from Reference [67]. 
\& Peters model considers only the static effect of thrust on the induced inflow distribu$\operatorname{tion}^{11}$.

As mentioned in Section 2.2.2.2, the Mangler and Squire inflow model is only applicable at advance ratios above approximately 0.1 . This was supported by the flapping amplitude results. Considering rotor side force and power coefficient would suggest that this inflow model is not well suited to the test conditions at advance ratios below approximately 0.15 .

Beyond the limitations of the prescribed inflow models already discussed in Section 2.2.2.2, additional differences between the simulated and experimental results may be attributed to the fuselage model used in the experiments, inaccuracies in the predicted airfoil coefficients, blade deformations and possible unreported airfoil trailing edge deflections.

Though no comparable experimental dataset was readily available for teetering rotors the same set of simulations was repeated using a soft in-plane teetering rotor hub. The precone angle was set to $3^{\circ}$ and the undersling distance was assumed zero. The number of blades was reduced from four to two and to keep the solidity constant the blade chord length was doubled leading to an assumed fourfold blade mass. Teetering moment of inertia and other dynamic parameters were adjusted respectively. The simulated results for this rotor assuming Drees and Mangler \& Squire inflow distributions are shown in Figure 3.11. The original experimental data for the fully articulated test rotor and the simulated results for the fully articulated rotor using the same inflow models are also included for reference.

\footnotetext{
${ }^{11}$ The full Pitt \& Peters inflow model also includes static effects on the inflow distribution due to rotor pitching and rolling moments[34]. These were not accounted for in the simulation, which may explain the residual error in the longitudinal and lateral disk deflection and rotor pitching and rolling moment estimations produced by the use of this model. Unsteady or dynamic effects of the Pitt \& Peters model were neglected as the simulation assumes steady airflow and control input conditions.
} 

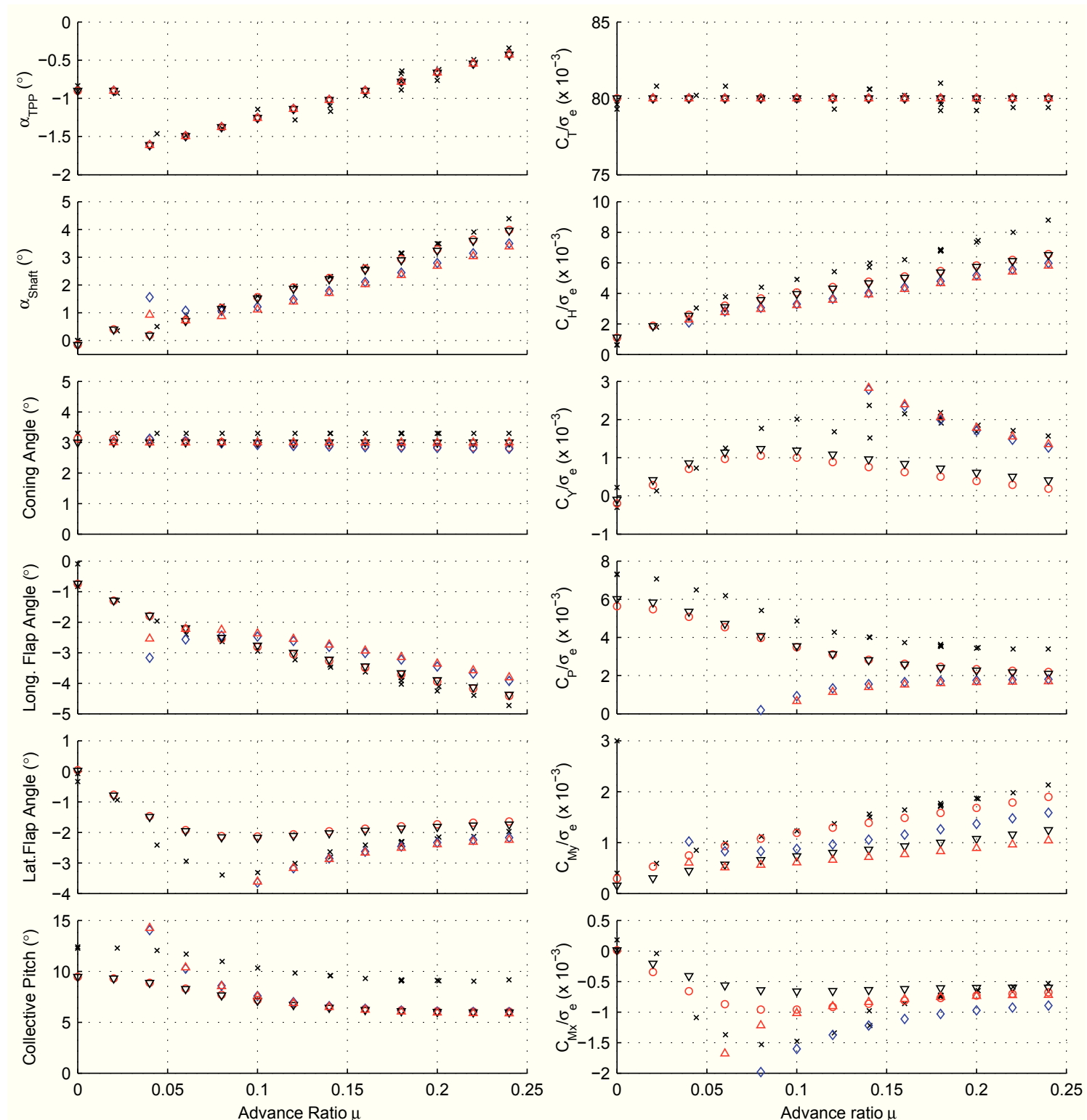

Experimental $\quad \circ \quad$ Drees(Art.) $\diamond \quad$ Mangler\&Squire(Art.)

Drees(Teet.)

Mangler\&Squire(Teet.)

Figure 3.11: Comparison of forward flight module simulated results to experimental data from Reference [67] (assuming teetering rotor hub). 
While a direct comparison of the results obtained for the teetering hub and the experimental results measured on a fully articulated test rotor hub could contain unknown degree of error, the lack of drastic differences was taken as a sign of confidence that the implementation of the equations of motion for the teetering rotor hub was not overtly erroneous. 


\section{Chapter 4}

\section{Rotor Blade Optimization}

As noted in Section 1.2, the objective of this work was the development of a simulation and optimization framework to aid in the performance driven aerodynamic design of $\mathrm{r} / \mathrm{c}$ helicopter rotor blades. This chapter outlines the integration of the Qoptr hover module with an optimization algorithm from the MATLAB Optimization Toolbox. The integration is verified using simplified analytical solutions of optimum rotor designs and a sample optimization for a rotor in hover is presented.

\subsection{Optimizer Integration Verification}

As discussed in Section 2.4.3 the MATLAB fmincon solver was selected as the optimization algorithm in this work. This function is documented in detail in Reference [68]. To demonstrate a viable integration of the simulation with the optimizer the hover module was selected to compare optimized designs to two theoretical idealized hover conditions presented in Reference [13, pp. 128-130, 135-138]. 


\subsubsection{The Ideal Twist Rotor}

Ideal blade twist for a rotor in hover has a well known analytical solution. A rectangular blade with constant lift curve slope $c_{l_{\alpha}}$ and constant drag coefficient $C_{d}$ is considered assuming no tip and root losses and neglecting airfoil coefficient variations due to compressibility or Reynolds number effects. Given these conditions, changes in profile power are negligible and maximum rotor efficiency is obtained by minimizing the induced power. As discussed in Section 2.2.1.1, induced power is minimized when the inflow distribution through the rotor disk is uniform. This can be shown to occur when the blade pitch distribution has the form shown in Equation 4.1 [13, p. 128].

$$
\theta(r)=\frac{\theta_{t i p}}{r}
$$

The optimization was set up to use negative figure of merit ${ }^{1}$ as the objective function and control values to define the pitch distribution at four radial locations were chosen as the design variables ${ }^{2}$. Figure 4.1 shows the results for a sample rotor.

Two optimized twist distributions are shown. In one case the blade pitch was linearly interpolated between the values of the control points. For the second case the control points were used to define a spline - more specifically a Bezier curve - to create a continuous spanwise pitch distribution. The optimized results returned the same value of figure of merit as the analytic solution to three and four decimal places using the linear interpolation and Bezier curve approaches, respectively.

\footnotetext{
${ }^{1}$ Negative figure of merit is specified, because the solver fmincon attempts to find the minimum of the objective function. The optimized design therefore represents a maximum figure of merit condition.

${ }^{2}$ Control values were defined at $r=0.1,0.25,0.4,0.7$ relative to the value at the blade tip.
} 

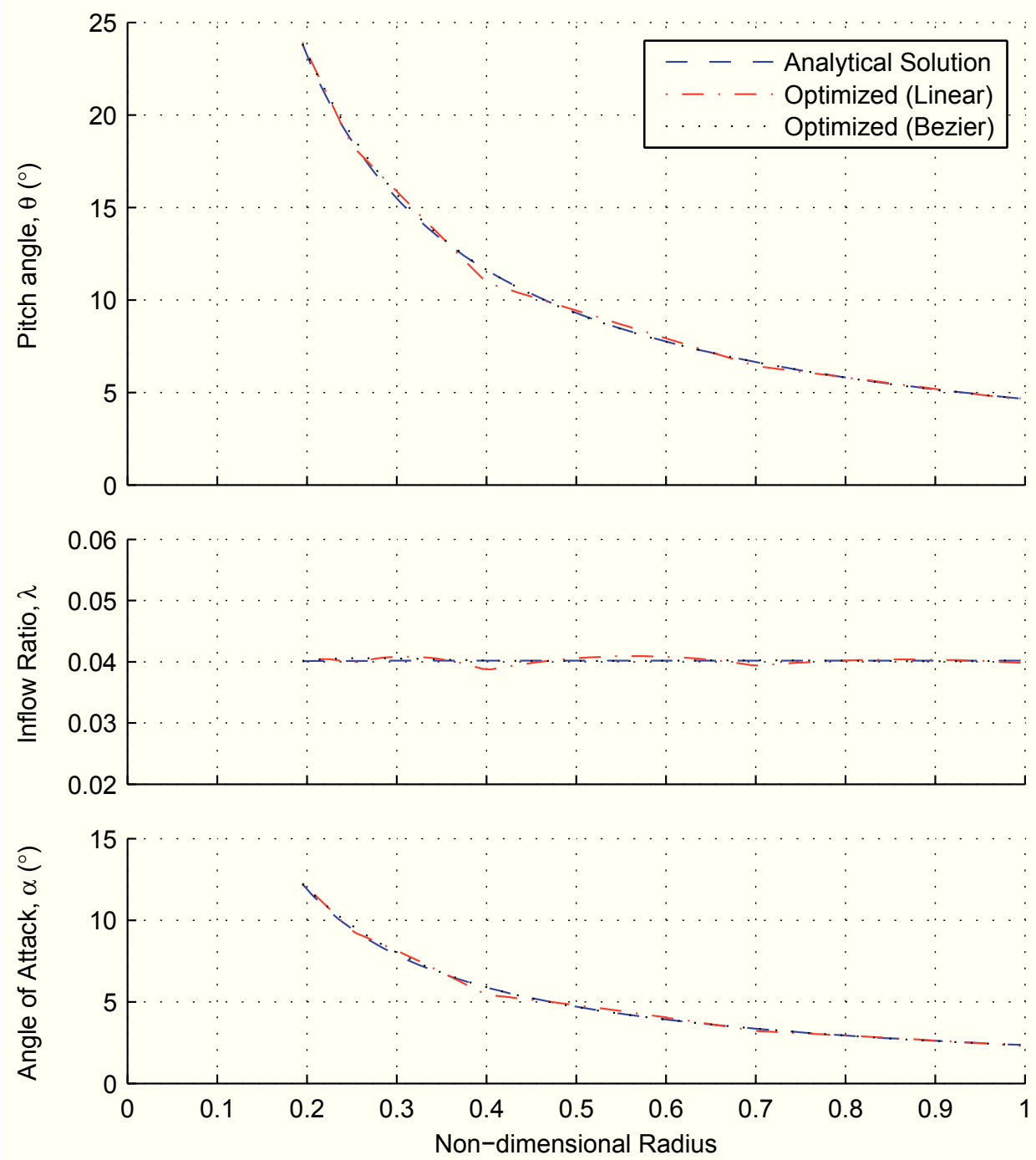

Figure 4.1: Optimization of twist distribution for a rotor with a rectangular planform.

\subsubsection{The "Optimum Hovering Rotor"}

Another analytically optimal design is referred to as the optimum hovering rotor [13, pp. 135-138]. For this design both induced and profile power are considered. The ideal twist rotor of the previous case results in a large range of angles of attack along the blade span. Varying both pitch and chord length (or solidity) it is possible to obtain a blade design that produces constant inflow ratio for minimum induced power while 
each blade section operates at the angle of attack for maximum airfoil lift to drag ratio $c_{l} / C_{d}$ - denoted $\alpha_{1}$ - to minimize profile power.

The required pitch and solidity distributions can be represented mathematically as shown in Equations 4.2 and 4.3 [13, pp. 136-137].

$$
\begin{gathered}
\theta(r)=\alpha_{1}+\frac{\lambda}{r}=\alpha_{1}+\sqrt{\frac{C_{T}}{2}} \frac{1}{r} \\
\sigma(r)=\frac{4 C_{T}}{C_{l_{\alpha}} \alpha_{1}} \frac{1}{r},
\end{gathered}
$$

This condition was simulated with the hover module using XFOIL generated airfoil data for the NACA 0012 airfoil at a Reynolds number of 200,000 which showed a maximum lift to drag ratio at $5^{\circ}$ angle of attack ${ }^{3}$. Reynolds number and compressibility effects were neglected and tip and root losses were not simulated. The optimized designs used negative figure of merit as the objective function. A total of nine control variables were utilized: Four defined each the distribution of pitch angle and solidity relative to their respective tip values. The ninth design variable allowed the equivalent rotor solidity to vary. The optimization was initialized with design variables representing a constant pitch, rectangular planform rotor blade with a solidity of 0.05 . The simulated analytical results as well as the two optimized designs - again differing only in the method used to interpolate between the control values - are shown in Figure 4.2.

The simulation of the analytical optimum hovering rotor design returned the expected constant inflow ratio and angle of attack distributions. The optimized designs both produced relatively constant angle of attack distributions at $\alpha_{1}$ as expected; however, the inflow distribution showed a decreasing trend with respect to radius. Further

\footnotetext{
${ }^{3}$ Airfoil coefficients were generated at $0.5^{\circ}$ intervals.
} 

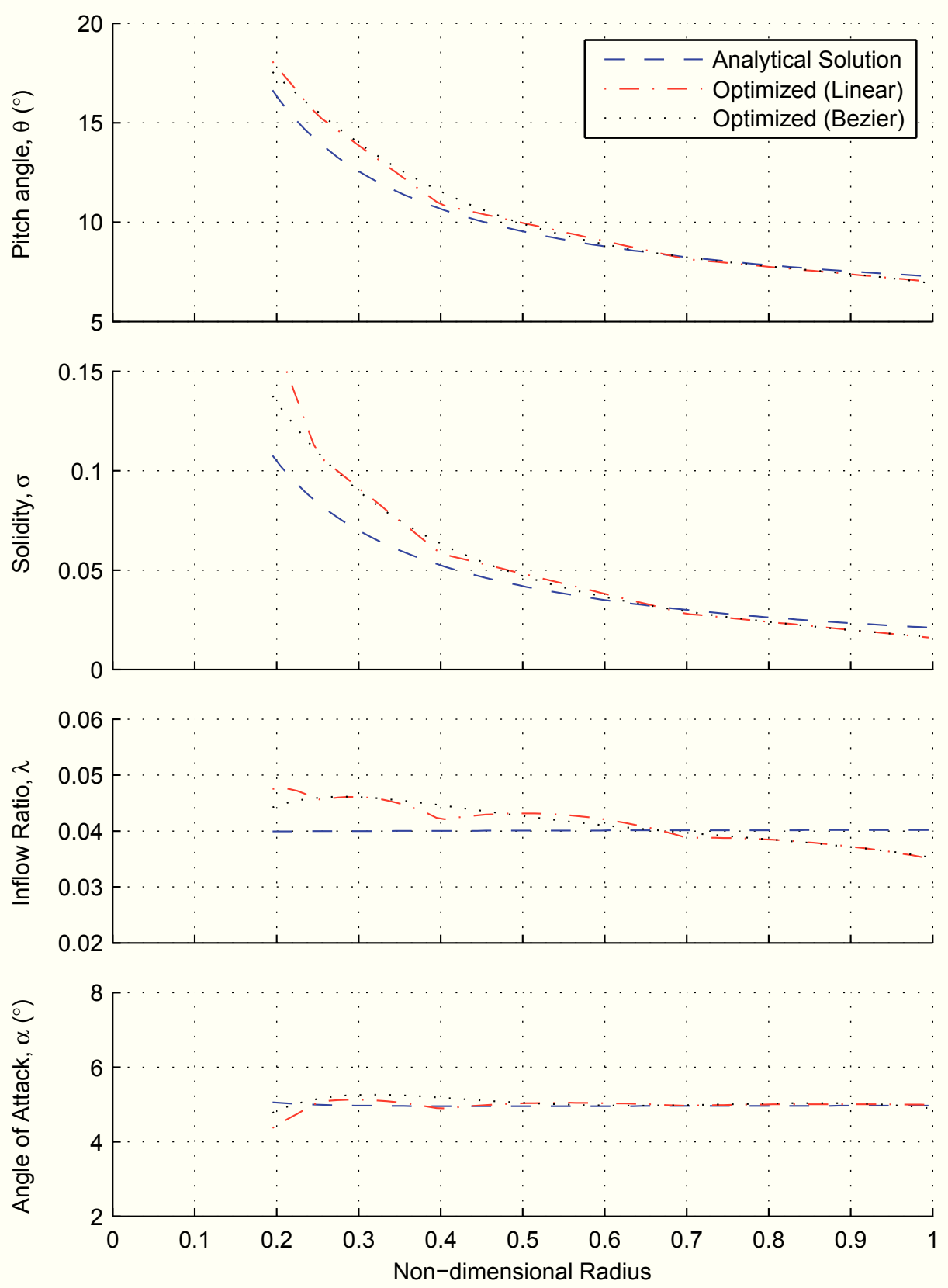

Figure 4.2: Optimization of twist and chord distributions in hover.

considering the performance parameters listed in Table 4.1, both optimized designs indeed showed a small increase in induced power coefficient for the optimized designs relative to the theoretical case. It seems that increasing the rotor solidity away from the 
Table 4.1: Simulated analytical and optimized results for the optimum hovering rotor design.

\begin{tabular}{cccccc}
\hline Case & $C_{T}$ & $C_{P_{i}}$ & $C_{P_{o}}$ & $\mathrm{FM}$ & $\sigma_{e}$ \\
\hline \hline Analytical Solution & 0.003118 & 0.000125 & 0.000045 & 0.7221 & 0.03032 \\
Optimized (Linear) & 0.003130 & 0.000127 & 0.000043 & 0.7274 & 0.03028 \\
Optimized (Bezier) & 0.003130 & 0.000127 & 0.000043 & 0.7274 & 0.03011 \\
\hline
\end{tabular}

rotor tip allowed this increase in induced power to be traded for a decrease in profile power, which resulted in a $0.5 \%$ increase in figure of merit relative to the analytical optimum design.

A specific reason to explain why the optimized design converged on a non-constant inflow distribution was not identified. However, the results were very close to each other and individual variations may have been masked by small differences in thrust coefficient and equivalent solidity. A possible explanation would be that figure of merit was more sensitive to profile power than induced power at the design point.

The results obtained for these two test cases were deemed sufficient evidence for a valid implementation of the Qoptr simulation module and MATLAB's fmincon optimization algorithm.

\subsubsection{Optimizer Limitations}

The selected optimizer fmincon requires the design variables and objective function and their first derivatives to be continuous. Therefore it is not able to select between different airfoils, which are discrete entities. One could, however, preselect for example two airfoils, one for the inboard region of the blade and the other for the tip. A design variable could then be used to define the radial distance at which the airfoil changes from the first to the second. 
Though the fmincon solver was the only optimization algorithm considered in this work, nothing speaks against the implementation of other types of optimization methods - including for example solvers based on genetic algorithms.

While the design variables passed from the optimizer to the objective function can be bounded and equality and inequality constraints in terms of these design variables can be specified, restrictions on other simulation parameters cannot be directly implemented as constraints in the optimizer. One way to still account for such constraints is the use of penalty functions in the objective function as shown in Section 4.2.2.

\subsection{Optimization of a 700 Class R/C Rotor for Hover}

\subsubsection{Model Rotor and Vehicle Description}

To demonstrate the performance improvement potential for $\mathrm{r} / \mathrm{c}$ helicopter rotors a baseline rotor was defined using values typical for a 700 class electric $\mathrm{r} / \mathrm{c}$ helicopter. In order to be representative of a UAS mission with a payload of sensors, communication equipment and extra energy storage a total vehicle mass of $8 \mathrm{~kg}$ was assumed. Ambient atmospheric conditions were taken at $250 \mathrm{~m} \mathrm{ISA}+15^{\circ}$. These baseline rotor parameter are summarized in Table 4.2. 
Table 4.2: Baseline rotor definition, loading and atmospheric operating conditions.

\begin{tabular}{lc}
\hline Parameter & Value \\
\hline \hline Rotor Definition & 2 \\
No. of Blades & $1.60 \mathrm{~m}$ \\
Diameter & $0.15 \mathrm{~m}$ \\
Root Cutout & $1500 \mathrm{rpm}$ \\
Rotorspeed & 0.05 \\
Equivalent Solidity & NACA 0012 \\
Airfoil & No Twist \\
Pitch Distribution & Rectangular \\
Blade Planform & \\
Vehicle and Loading & $8.0 \mathrm{~kg}$ \\
Total Takeoff Mass & $78.5 \mathrm{~N}$ \\
Total Takeoff Weight & 1.05 \\
Main Rotor Blockage & \\
Atmospheric Conditions (ISA $\left.250 \mathrm{~m}+15^{\circ}\right)$ & $1.136 \mathrm{~kg} / \mathrm{m}^{3}$ \\
Density & $1.85 \times 10^{-5} \mathrm{~Pa}-\mathrm{s}$ \\
Viscosity & $348.1 \mathrm{~m} / \mathrm{s}$ \\
Speed of Sound &
\end{tabular}

\subsubsection{Optimization Constants, Variables and Constraints}

During the optimization cases presented in Section 4.2, certain rotor parameters were held constant while others were free to change. The decisions made as to whether a certain rotor parameter should be allowed to be controlled by the optimization algorithm were based on two main factors: the ability to integrate the design variable in the fmincon optimization function and limiting the amount of secondary vehicle modifications required as a result of changing from one set of rotor blades to another. 
For example, the selection of airfoils and the amount of blades involve discrete design variables, which are not compatible with gradient based optimization algorithms like fmincon. Airfoils were preselected outside of the optimization loop as described in Section 4.2.3 and the number of rotor blades was kept constant at two. Rotor diameter was also held constant; while small changes may have been possible, larger variations could lead to physical interference issues with vehicle tail components.

Rotor speed on the other hand was allowed to vary as it is expected to be relatively simple to program different speeds into the vehicle control system. Parameters that define the shape of the rotor blade were also open to be used as design variables. These include the rotor solidity as well as spanwise pitch and chord distributions.

Certain parameters were limited to remain within practical envelopes. It should be noted that no attempt was made to simulate or otherwise evaluate internal rotor blade structural and dynamic loading conditions and that the following limits were set on a qualitative "common sense" basis and may therefore not represent real structural or dynamic limitations. A lower limit on rotor speed was set at $1250 \mathrm{rpm}$, which had been identified by a UAS operator to represent the approximate condition at which a 700 class rotor becomes increasingly sensitive to wind gusts. Chord length was limited to a maximum of $0.08 \mathrm{~m}$ to prevent very large - and therefore heavier - chords at the blade root. Chord length at blade stations smaller than 0.8 were also limited to a minimum of $0.05 \mathrm{~m}$ and beyond blade station 0.8 to $0.04 \mathrm{~m}$ to keep the blade from becoming too slender and maintain stiffness requirements. For reference, commercial rotor blades for a 700 class electric $\mathrm{r} / \mathrm{c}$ helicopter commonly have a chord length of approximately 0.060 to $0.065 \mathrm{~m}$.

As the limits placed on chord length may not be easily expressed in terms of the design variables - depending on the interpolation method used - penalty functions 
were added to the objective function as shown in Equation 4.4

$$
f=f_{\text {orig }}+\gamma_{1} P_{1}+\gamma_{2} P_{2}+\ldots
$$

where $P_{1}, P_{2}$, etc. individually represent penalty functions related to one of the limits [52, pp. 445-446]. For example, the maximum chord length limit penalty function is defined as the square of the sum of excess chord amounts of all blade sections. Therefore, if the chord at any blade section is larger than the limit of $0.08 \mathrm{~m}$, the objective function returns a slightly larger result than the direct negative of the figure of merit and the optimizer attempts to minimize the sum of these terms. Penalty functions do not strictly enforce the specified limits as the optimizing algorithm does not consider them directly, but constants $\gamma_{1}, \gamma_{2}$, etc. may be adjusted to keep the limit violations small.

\subsubsection{Selection of Airfoils}

As discussed in Section 2.3.3, there has been very limited rotorcraft airfoil development in the lower Reynolds number range. And since it is beyond the scope of this work to develop new airfoil profiles for the conditions observed for $r / c$ helicopters, it was decided to evaluate a selection of existing rotorcraft airfoils at these conditions using the numerical method selected in Section 3.1 to generate airfoil performance coefficients. Based on various airfoil criteria - such as high airfoil lift to drag ratio over specific ranges of angle of attack or low pitching moment magnitude - airfoils with the desired characteristics may then be selected. 
Figure 4.3 shows the maximum airfoil lift coefficient for rotorcraft airfoils at four different Reynolds numbers. The displayed values assume incompressible flow and transition parameter $n_{\text {crit }}=9$. Airfoil profile coordinates were obtained from References [63] and [22]. As noted in Section 3.1.2, XFOIL generated airfoil data is not necessarily very accurate especially near stall, which is where these maximum lift coefficient values were obtained.

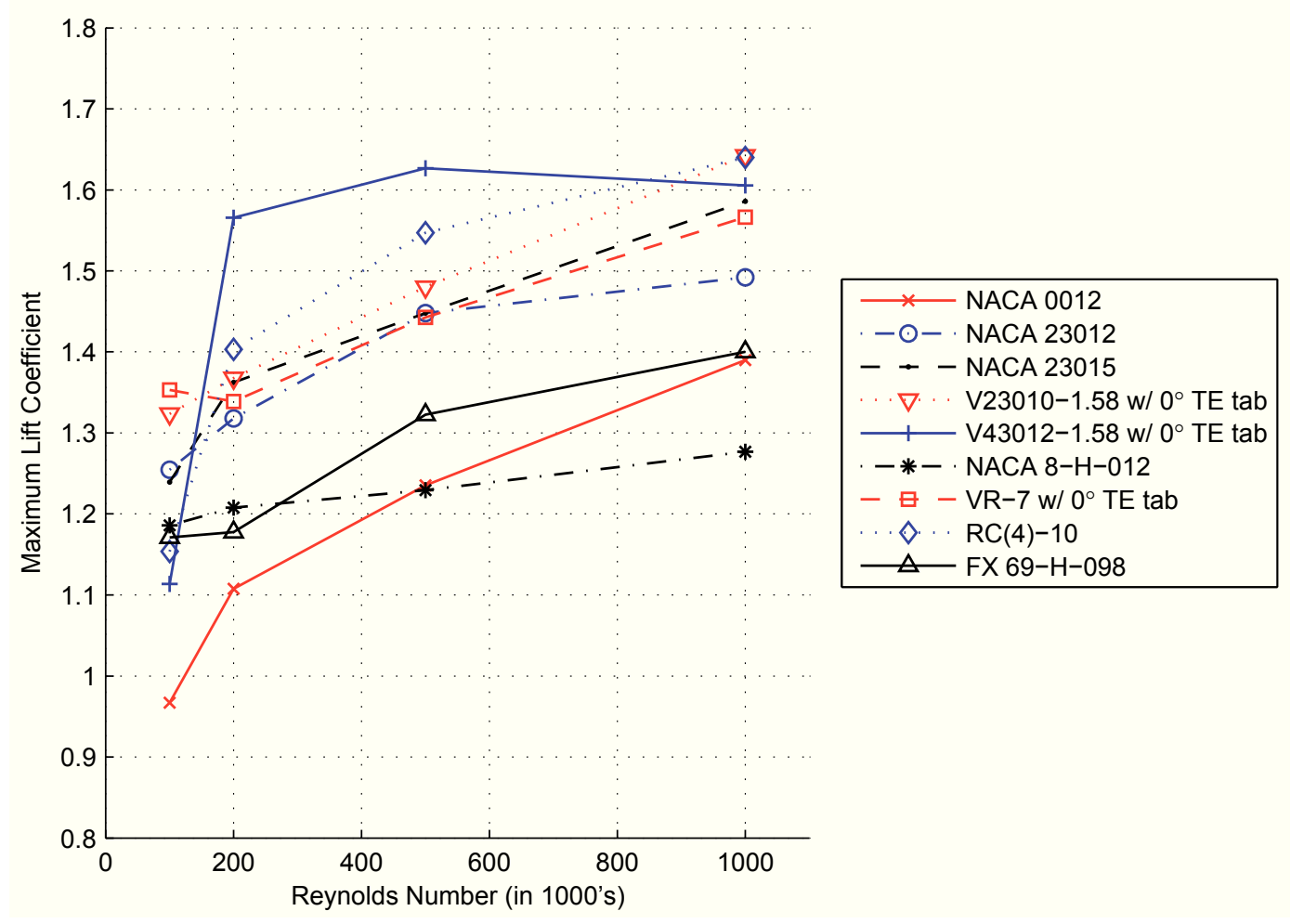

Figure 4.3: Maximum lift coefficient of rotorcraft airfoils at Reynolds numbers between 100,000 and $1,000,000$.

Figure 4.4 depicts the maximum section lift to drag ratios for the same airfoils shown in Figure 4.3. While the XFOIL generated drag coefficients were also found to be optimistic in the higher angle of attack range (see Section 3.1.2), the maximum lift to drag ratio is most often achieved at an angle of attack below stall, which suggests that these results align better with reality. 


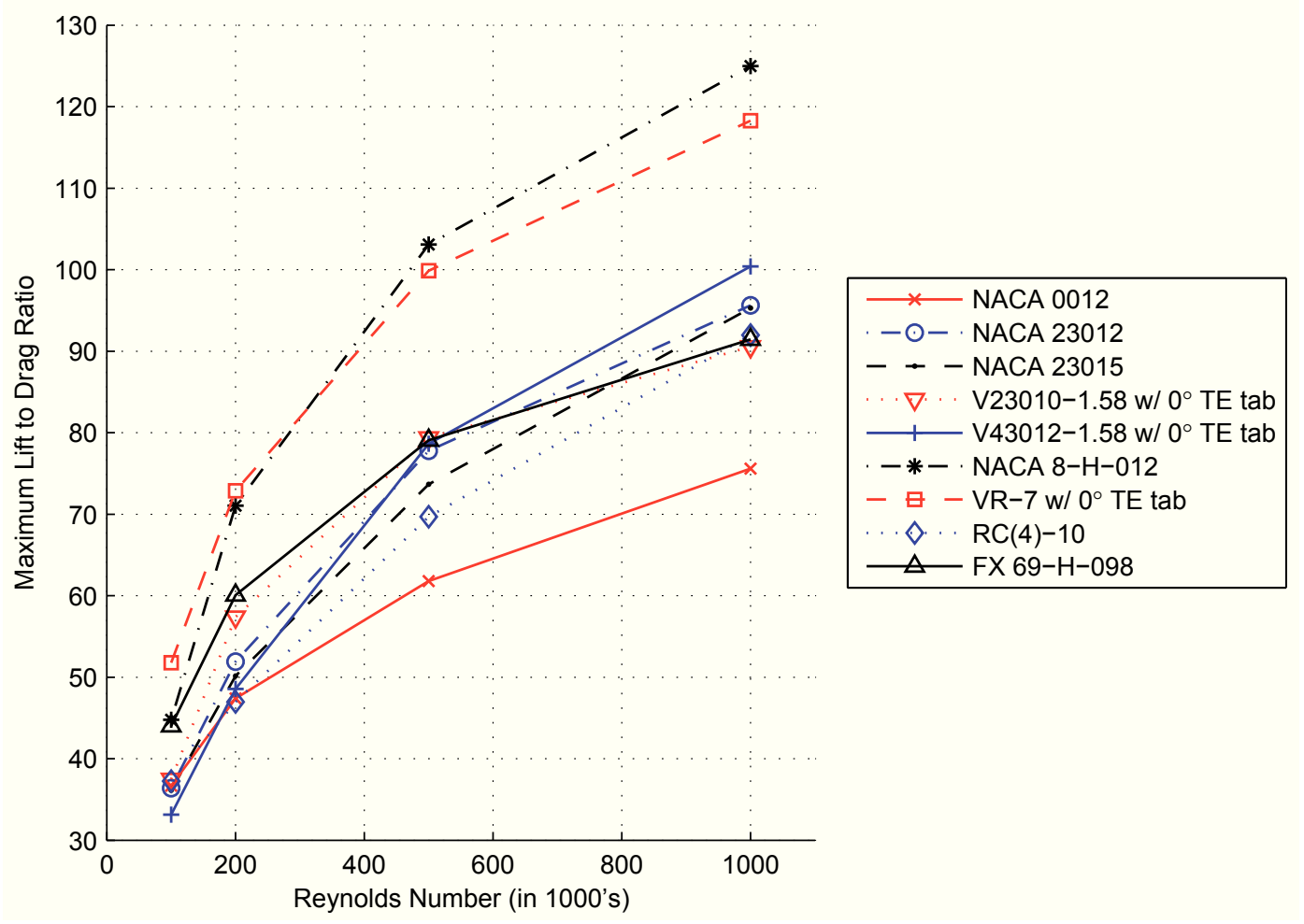

Figure 4.4: Maximum lift to drag ratio of rotorcraft airfoils at Reynolds numbers between 100,000 and 1,000,000.

Considering the trends shown in Figures 4.3 and 4.4 most of the shown airfoils' maximum lift coefficients vary significantly with Reynolds number. Changes in maximum lift coefficient between Reynolds numbers of 200,000 and 1,000,000 are largely limited to approximately 0.2 for individual airfoils. Below a Reynolds number of 200,000 some airfoils' maximum lift coefficient drop dramatically, while others are not affected significantly and the maximum lift coefficient of the VR-7 airfoil even shows a small increase. The trends in maximum airfoil lift to drag ratio are more common between different airfoil. An increasing trend with decreasing sensitivity with respect to Reynolds number is observed for all airfoils shown.

In both cases - considering maximum lift coefficient and maximum lift to drag ratio - the NACA 0012 airfoil performs relatively poorly, which is not surprising when a 
symmetric airfoil is compared with cambered ones. However, these two figures also demonstrate some of the complications involved when choosing a suitable airfoil. While the maximum lift coefficient of the NACA 8-H-012 was relatively low, its lift to drag ratio outperformed the other airfoils - most by a large margin.

Since the data displayed in Figures 4.3 and 4.4 represent values at individual values of angle of attack, an informed airfoil selection requires a more in depth analysis of the variations of these and other parameters over significant ranges in angle of attack and Reynolds number, especially if the rotor is to operate in non-zero advance ratio conditions. Appendix E contains numerical data for a slightly larger selection of rotorcraft airfoils at the maximum lift coefficient and maximum lift to drag ratio including the corresponding angles of attack and pitching moment values.

For the examples shown in this chapter, only one airfoil profile will be used along the blade span.

\subsubsection{Hover Optimization Results}

As described in the preceding sections, an optimization routine using the negative of the figure of merit as the objective function was set up to find a rotor blade design for a $1.6 \mathrm{~m}$ diameter rotor in hovering flight. A total of ten design variables were defined: One each for rotor speed and equivalent solidity and four each for spanwise variations in blade pitch and chord length. Parameters were limited as outlined in Section 4.2.2. Rotor thrust was held constant by adjusting the thrust coefficient $C_{T}$ according to changes in rotor speed. The blade pitch and chord length distributions were defined using continuous Bezier curves with radial control point stations at $\mathrm{r}=$ $0.15,0.45,0.7,0.9$, relative to the respective tip values. Two optimized results are presented, differing only in the airfoil used: The first utilized the $\mathrm{RC}(3)-10$ and the 
second the RC(4)-10 airfoil. These airfoils were selected as both feature relatively high airfoil lift to drag ratios at low values of angle of attack in the Reynolds number range from 100,000 to 200,000 .

Table 4.3 lists a number of numeric results obtained for the three rotor designs. Spanwise distributions of a number of rotor parameters are shown in Figure 4.5, as simulated at the design thrust value.

Table 4.3: Performance parameters for the baseline and optimized rotor at the design point.

\begin{tabular}{cccc}
\hline Parameters & Baseline & Opt. RC(3)-10 & Opt. RC(4)-10 \\
\hline \hline FM & 0.5556 & 0.7000 & 0.6985 \\
Thrust (N) & 82.42 & 82.42 & 82.42 \\
Power (W) & 630.1 & 500.1 & 501.2 \\
$C_{T}$ & 0.002285 & 0.003287 & 0.003289 \\
$C_{P_{i}}$ & $8.747 \times 10^{-5}$ & $1.411 \times 10^{-4}$ & $1.414 \times 10^{-4}$ \\
$C_{P_{o}}$ & $5.155 \times 10^{-5}$ & $4.933 \times 10^{-5}$ & $4.959 \times 10^{-5}$ \\
$\sigma_{e}$ & 0.050 & 0.0378 & 0.0428 \\
$\Omega(\mathrm{rpm})$ & 1500 & 1250.6 & 1250.2 \\
$M_{\text {Tip }}$ & 0.361 & 0.301 & 0.301 \\
\hline
\end{tabular}

Immediately noticeable is an approximate 0.14 increase in figure of merit from the baseline rotor to the two optimized designs, providing the same amount of thrust at approximately $80 \%$ of the power required by the baseline rotor. Thrust and power coefficients are not directly comparable due to the difference in rotor speed, but considering the ratios of induced power to profile power of the individual designs a significant reduction in profile power becomes evident. As also seen in Figure 4.5 the rotor solidities of the optimized rotor both gravitated to lower values; the distribution of solidity (or chord length) along the blade and the equivalent solidity value were heavily influenced 

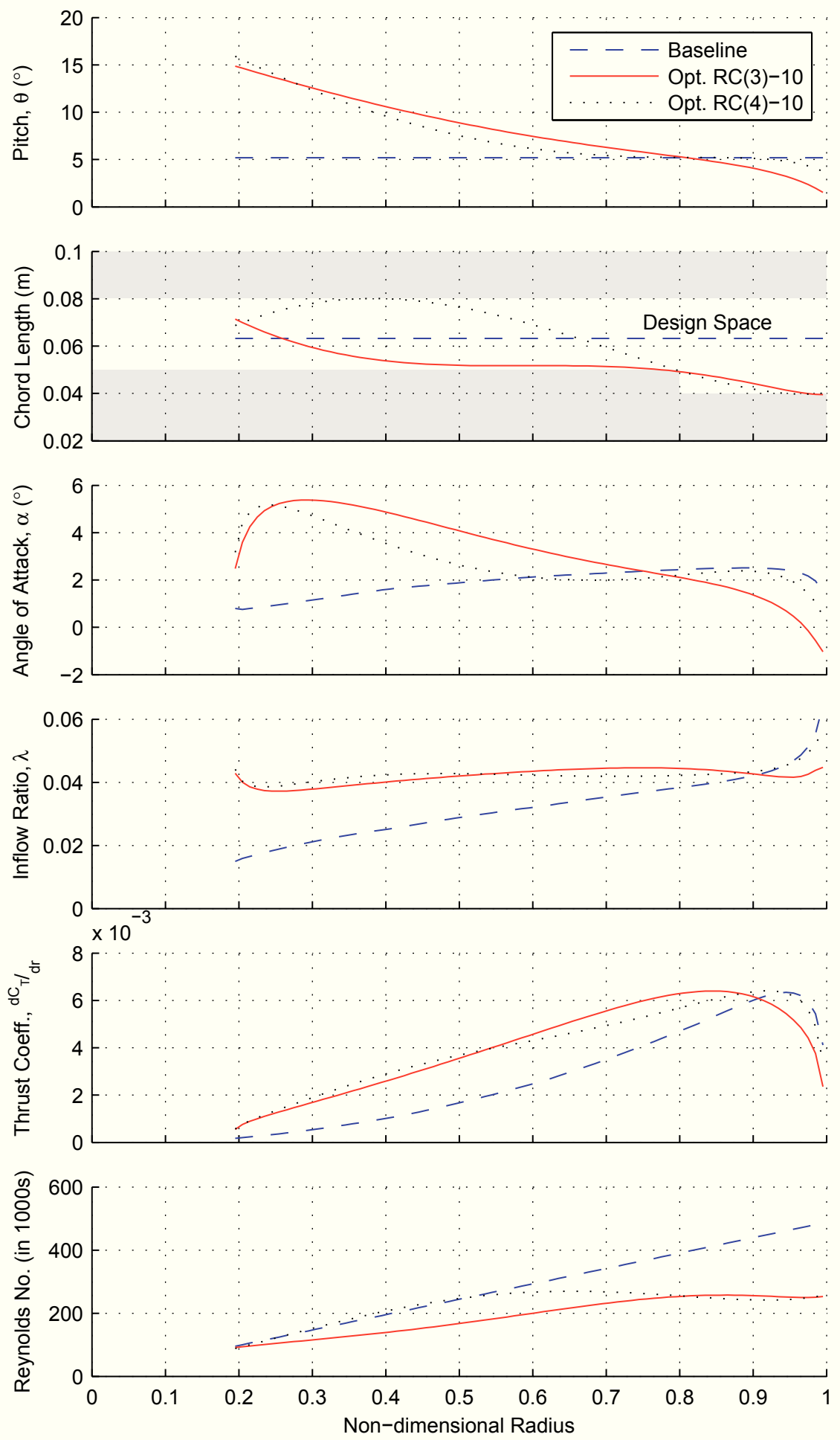

Figure 4.5: Radial performance parameter variations at the design point. 
by the airfoil used. In both optimizations the rotor speed trended toward the lower limit at $1250 \mathrm{rpm}^{4}$. This resulted in a relatively low tip Mach number near 0.3 and suggests that compressibility effects on $\mathrm{r} / \mathrm{c}$ rotors of this size and under similar loading and operating conditions are not significant.

Both optimized designs featured negative twist on the order of 15 to $20^{\circ}$ with a drop-off near the tip. The chord distributions were significantly different for the two airfoils. The RC(3)-10 airfoil has an optimum lift to drag ratio near 4.5 to $5.0^{\circ}$ angle of attack at Reynolds numbers from 100,000 to 200,000. Under the same conditions, the $\mathrm{RC}(4)-10$ has its maximum lift to drag ratio near 2.5 to $3.5^{\circ}$ angle of attack. Since the rotor with the RC(3)-10 airfoil operates more optimally at higher angles of attack, the chord length distribution 'hugs' the lower constraint, resulting in an overall lower rotor solidity.

The inflow ratio distributions of the two optimized rotors were significantly more level than that of the baseline rotor and the thrust coefficient distribution of the optimized designs were more triangular compared to the distribution on the baseline rotor. Both of these characteristics lead to a more efficient distribution of thrust generation around the rotor disk and a reduction in the induced power requirement.

The Reynolds number distributions show a significant reduction, especially near the tip, when the optimized designs are compared to the baseline rotor. This is caused by the reduction in rotor speed and chord length. The hover operating Reynolds number range is thus placed between 100,000 and 300,000, which is the lower end of the range first considered during the development of the simulation algorithms.

Figure 4.6 shows the off-design performance of the three rotor designs: Figure of merit, total rotor power and power loading - the amount of thrust generated per unit

\footnotetext{
${ }^{4}$ The small offset of the value of rotor speed from the limit at $1250 \mathrm{rpm}$ may have been caused by the interior point search method selected for the optimization algorithm.
} 
of power used - are plotted with respect to rotor thrust. It can be seen that while the design point does not coincide with the figure of merit maxima for any of the rotors, the optimized designs operate significantly closer to their maximum figure of merit operating conditions. The simulated hover thrust stall margins are large on all three
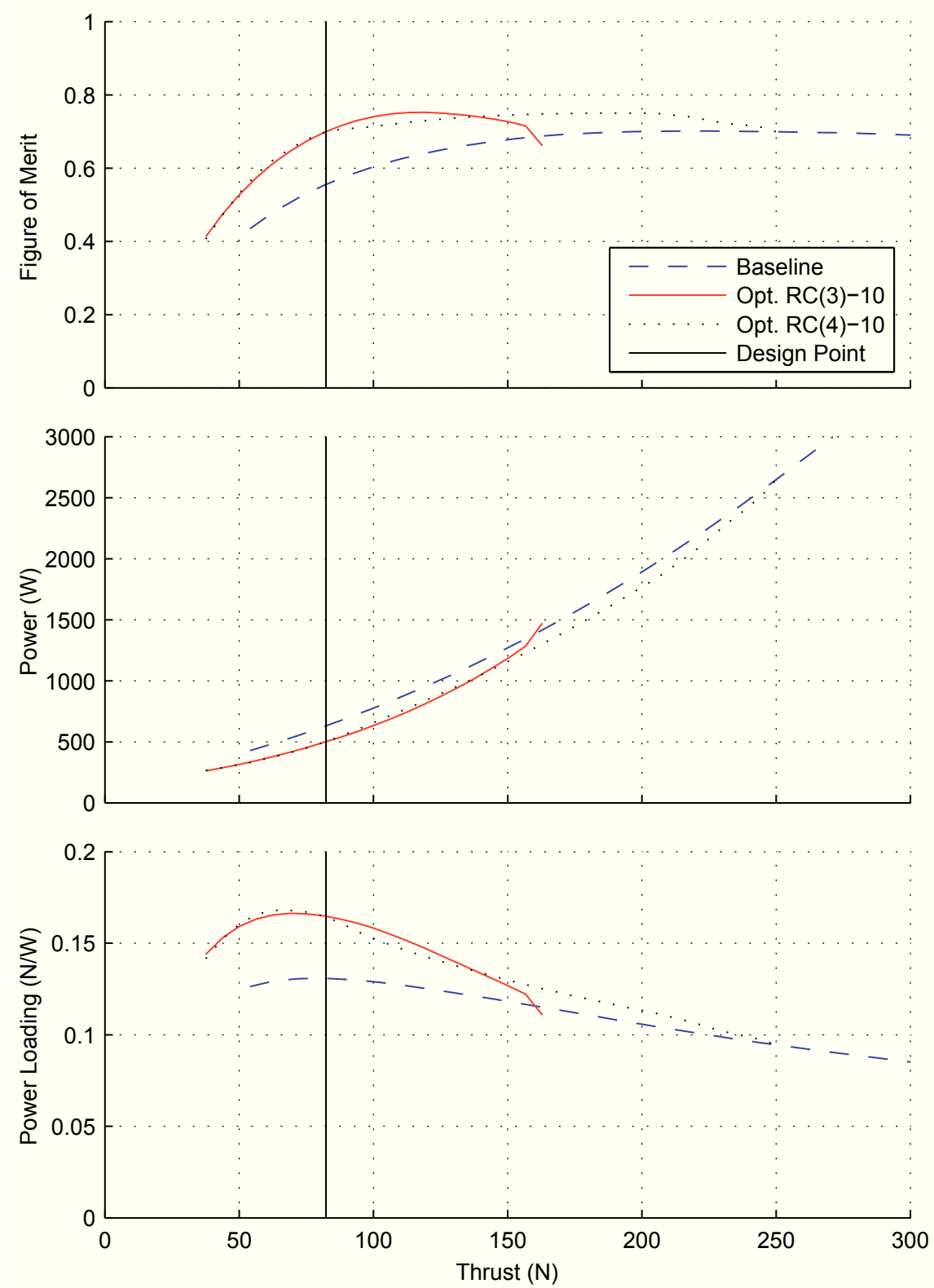

Figure 4.6: Rotor performance at off-design conditions. 
rotor designs: The optimized $\mathrm{RC}(3)-10$ and the $\mathrm{RC}(4)-10$ rotors were simulated to stall at approximately $160 \mathrm{~N}$ and $245 \mathrm{~N}$, respectively, which exceeded the design thrust level in hover by about 100 and $200 \%$, respectively. The baseline rotor was simulated to stall near $360 \mathrm{~N}$, exceeding the hover level by approximately $340 \%$. The power and power loading graphs also show significant performance improvements for the optimized rotor designs at essentially all operating conditions before the aforementioned stall limits.

A number of operational performance parameters for the optimization runs are listed in Table 4.4. Starting the optimization from the initial conditions specified above - untwisted and rectangular blades with a rotor solidity of 0.05 and a rotor speed of $1500 \mathrm{rpm}$ - the optimizer required on the order of 150 iterations to converge to the final design, at which relative changes to the design variables were less than $10^{-6}$. During the optimization 1300 to 2000 calls to the objective function were made, each including a call to the hover module simulation. On a laptop computer with a $2.67 \mathrm{GHz}$ Intel Core i5 processor and $4 \mathrm{~Gb}$ of RAM the optimizations took about 5 minutes to complete individually. Initiating the optimization run with a $-5^{\circ}$ linear twist, linear root-to-tip taper ratio of 1.5 and the same rotor solidity and rotor speed as before reduced the time requirement by about $20 \%$ while returning final equivalent solidity and rotor speed values within $0.2 \%$ and standard deviations in pitch and chord distributions within $0.02^{\circ}$ and $0.2 \mathrm{~mm}$ compared to the results of the original optimization runs.

Table 4.4: Optimizer performance evaluation.

\begin{tabular}{ccccc}
\hline Case & $\begin{array}{c}\text { Starting Conditions } \\
\text { Twist / Taper }\end{array}$ & $\begin{array}{c}\text { Optimizer } \\
\text { Iterations }\end{array}$ & $\begin{array}{c}\text { Function } \\
\text { Evaluations }\end{array}$ & $\begin{array}{c}\text { Time } \\
\text { (s) }\end{array}$ \\
\hline \hline \multirow{2}{*}{ Opt. RC(3)-10 } & None / Rectangular & 109 & 1347 & 286 \\
& $-5^{\circ} / 1.5: 1$ & 111 & 1315 & 235 \\
Opt. RC(4)-10 & None / Rectangular & 159 & 1924 & 300 \\
& $-5^{\circ} / 1.5: 1$ & 149 & 1805 & 233 \\
\hline
\end{tabular}




\section{Chapter 5}

\section{Conclusions and Recommendations}

\subsection{Conclusions and Accomplishments}

The Qoptr framework was developed to simulate the aerodynamic performance of $\mathrm{r} / \mathrm{c}$ helicopter rotors and to optimize the performance at a specific operating condition through changes in the rotor blade design. Two simulation modules were constructed in MATLAB: one to simulate hover using blade element momentum theory and the second to model forward flight performance using a blade element theory approach. Both, the hover module and the forward flight module were successfully validated through individual comparisons to published experimental results. The simulation results consistently underpredicted the required rotor power when numerically generated airfoil data was used. The use of experimentally determined airfoil performance data mostly rectified the simulation error at low to medium loading conditions; however, at high loading conditions the simulation results remained optimistic.

Of the two 2D airfoil performance data generating programs evaluated in this work XFOIL proved to provide better results at significantly higher speed. Especially at high airfoil loading conditions near stall, however, XFOIL delivered optimistic results with maximum lift coefficients exceeding and drag coefficients underestimating the actual 
airfoil parameters. The VII package could not be used to predict airfoil stall without prior knowledge of the stall behaviour and was therefore deemed inadequate for the task at hand. Even after the development of an empirical correction model for the computed lift coefficient the predictions of the VII algorithm were shown to be less reliable than those produced by XFOIL.

The results of the sample optimization run suggest that significant efficiency improvements can be expected for rotorcraft UAS through the use of rotor blades specifically designed for the prevalent flight condition required for the mission. The predicted $20 \%$ reduction in power could, for example, be directly related to a $25 \%$ increase in hovering endurance.

\subsection{Recommended Future Extensions for the Qoptr Framework}

As the development of the simulation and optimization framework as a whole was the primary focus of this work a number of "problematic" areas were set aside for more detailed treatment in the future. Already noted in the previous section is the deficiency in the simulation results seen due to the use of numerically generated airfoil data compared to experimental airfoil data. Different numerical methods - such as computational fluid dynamics - may be evaluated to enable the generation of more reliable coefficients for the Qoptr airfoil data look-up tables, when higher accuracy simulations are required. Though the sample optimization suggests that compressibility effects are not a major influence on the efficiency operation of lightly loaded $r / c$ helicopter rotors, a method able to account for Mach number effects on the airfoil drag coefficient could be implemented for more highly loaded scenarios. 
Potential improvements to the forward flight module include the implementation of methods to account for unsteady aerodynamic effects that build on the airfoil coefficients provided by the steady state data look-up tables and the possible incorporation of an empirical dynamic stall model. A more detailed treatment of the reversed flow region is also advised. The derivation and implementation of a flexible blade model would improve the realism of the simulated blade angles, enable the estimation of stresses and vibratory loads and allow the direct incorporation of bearingless ${ }^{1}$ rotor hub configurations.

These features are expected to improve the simulation results, especially at extreme disk loading conditions and high advance ratios; they will, however, also increase the computational requirements of the algorithm.

Lastly, similar to the optimization using the MATLAB function fmincon and the Qoptr hover module presented in Section 4.2, objective functions calling the forward flight module can be established to optimize the rotor blade performance for a specific flight speed. Other types of optimization algorithms may also be incorporated in order to enable the use of discrete design variables and objective functions may be designed to account for rotor performance at more than a single flight condition. One must keep in mind, however, that the optimization setup can vary greatly from one set of goals, design variables and constraints to the next and will therefore always require manual input from the design engineer.

\footnotetext{
${ }^{1}$ Bearingless or rigid rotor hub configurations do not feature dedicated flap, lead-lag and feathering hinges. Instead the blade motion is accommodated by flexures in the rotor hub and the flexibility of the rotor blades themselves. Refer also to the discussion on alternative rotor hub designs in Appendix C.
} 


\section{List of References}

[1] Teal group predicts worldwide UAV market will total $\$ 89$ billion in its 2013 UAV market profile and forecast, Teal Group Corporation: Aerospace and Defense Market Intelligence - Analysis and Forecasts, Jun. 17, 2013, [Online]. Available: http : / / tealgroup . com/index . php/about - teal - groupcorporation / press - releases / 94 - 2013 - uav - press - release (Accessed Jun. 19, 2014).

[2] D. Chabot, "Precise, convenient and unobtrusive wildlife and habitat monitoring using small UAS," CASI Ottawa Branch Meeting, Carleton University, Mar. 31, 2014.

[3] CAMCOPTER S-100 - System, Schiebel, [Online]. Available: http : //www . schiebel .net/Products/Unmanned-Air-Systems/CAMCOPTER-S-100/ System. aspx (Accessed Jun. 19, 2014).

[4] A160 Hummingbird: Boeings variable-rotor VTUAV, Defense Industry Daily, Jul. 4, 2012, [Online]. Available: http: //www . defenseindustrydaily . com / a160 - hummingbird - boeings - variable - rotor - vtuav - 03989/ (Accessed Jun. 20, 2014).

[5] MQ-8B Fire Scout data sheet, Northrop Grumman, [Online]. Available: http: //www . northropgrumman. com/Capabilities/FireScout/Documents/ 
pageDocuments/MQ-8B_Fire_Scout_Data_Sheet.pdf (Accessed Jun. 19, 2014).

[6] MQ-8C Fire Scout data sheet, Northrop Grumman, [Online]. Available: http: //www . northropgrumman . com/Capabilities/FireScout/Documents/ pageDocuments/MQ-8C_Fire_Scout_Data_Sheet.pdf (Accessed Jun. 19, 2014).

[7] G. Warwick, "Automated resupply," Aviation Week \& Space Technology, vol. 175, no. 28, p. 36, Aug. 2013.

[8] A. Osborne, "Optionally piloted helo tests," Aviation Week \& Space Technology, vol. 175, no. 14, pp. 14-16, Apr. 2013.

[9] Helicopters - helicopter kits, HeliDirect, [Online]. Available: http : //www . helidirect . com/helicopters - helicopter-kits-c-1_29.hdx (Accessed Jun. 19, 2014).

[10] Great hobbies R/C helicopter department, [Online]. Available: http : / www . greathobbies. com/rchelicopters/ (Accessed Jun. 19, 2014).

[11] Banshee 700 limited, Banshee Helicopters, [Online]. Available: http://www . banshee-helicopters . de/index .php/en/shoppingcart/product/ view/1/13 (Accessed Jun. 19, 2014).

[12] Responder, ING Robotic Aviation, [Online]. Available: http://ingrobotic . com/aircrafts/rotorcraft-responder/ (Accessed Jun. 19, 2014).

[13] J. G. Leishman, Principles of Helicopter Aerodynamics, 2nd. Cambridge, UK: Cambridge University Press, 2006. 
[14] M. Fadely. Helicopter stunts, Model Aviation, [Online]. Available: http://www . modelaviation. com/helicopterfadely (Accessed Nov. 1, 2013).

[15] SpinBlades - high performance premium blades, SpinBlades, [Online]. Available: http://www . spinblades . com/ (Accessed Nov. 13, 2013).

[16] B. H. Carmichael, "Low Reynolds number airfoil survey, Volume I," NASA Langley Research Center, Hampton, VA, NASA CR 165803, 1981.

[17] C. A. Lyon, A. P. Broeren, P. Giguere, A. Gopalarathnam, and M. S. Selig, Summary of low-speed airfoil data. Virgina Beach, VA: SoarTech Publications, 1998, vol. 3.

[18] D. Althaus and F. X. Wortman, Stuttgarter Profilkatalog I. Braunschweig, Wiesbaden: Vieweg, 1981.

[19] D. Althaus, Stuttgarter Profilkatalog II - Niedriggeschwindigkeitsprofile. Braunschweig, Wiesbaden: Vieweg, 1996.

[20] Rotorblätter / Paddel - Hauptrotor - 680 - 720, World of Heli, [Online]. Available: http: / /www . world-of -heli.de/rotorblaetter/hauptrotor/ 690-710-mm/index.html (Accessed Jun. 20, 2014).

[21] A. E. Phelps and S. L. Althoff, "Effects of planform geometry on hover performance of a 2-meter-diameter model of a four-bladed rotor," NASA Langley Research Center, Hampton, VA, NASA TM 87607, AVSCOM TR 85-B-6, 1986.

[22] S. L. Althoff, "Effect of advanced rotorcraft airfoil sections on the hover performance of a small-scale rotor model," NASA Langley Research Center, Hampton, VA, NASA TP 2832, AVSCOM TP 88-B-001, Sep. 1, 1988. 
[23] S. L. Althoff and K. W. Noonan, "Effect of blade planform variation on a smallscale hovering rotor," NASA Langley Research Center, Hampton, VA, NASA TM 4146, AVSCOM TM 89-B-009, 1990.

[24] B. Berry, G. Bowen-Davis, D. Mayo, D. Pfeifer, V. Posbic, A. Sridharan, I. Chopra, J. G. Leishman, and V. Nagaraj, "Goliath twin-lift system," University of Maryland, Alfred Gessow Rotorcraft Center, AHS Student Design Competition Report, 2010. [Online]. Available: https : / / vtol . org / download . cfm? downloadfile =4CB30950 - E859 - 11E0 - AE6C0050568D0042 \& typename = dmFile\&fieldname=filename (Accessed Nov. 11, 2013).

[25] B.-E. Lee, Y.-S. Byun, J. Kim, and B.-S. Kang, "Experimental hover performance evaluation on a small-scale rotor using a rotor test stand," Journal of Mechanical Science and Technology, vol. 25, no. 6, pp. 1449-1456, 2011.

[26] L. A. Young, E. W. Aiken, J. L. Johnson, R. Demblewski, J. Andrews, and J. Klem, "New concepts and perspectives on micro-rotorcraft and small autonomous rotary-wing vehicles," presented at the 20th AIAA Applied Aerodynamics Conference, St. Louis, MO, 2002.

[27] B. R. Hein and I. Chopra, "Hover performance of a micro air vehicle: Rotors at low Reynolds number," Journal of the American Helicopter Society, vol. 52, no. 3, pp. 254-262, 2007.

[28] F. Bohorquez, P. Samuel, J. Sirohi, D. Pines, L. Rudd, and R. Perel, "Design, analysis and hover performance of a rotary wing micro air vehicle," Journal of the American Helicopter Society, vol. 48, no. 2, pp. 80-90, 2003. 
[29] F. Bohorquez, D. Pines, and P. D. Samuel, "Small rotor design optimization using blade element momentum theory and hover tests," Journal of Aircraft, vol. 47, no. 1, pp. 268-283, 2010.

[30] W. Johnson, Helicopter Theory. New York, NY: Dover Publications, 1994.

[31] J. M. Seddon and S. Newman, Basic Helicopter Aerodynamics, 3rd ed. Chichester, United Kingdom: John Wiley \& Sons, 2011.

[32] A. Betz, "Schraubenpropeller mit geringstem Energieverlust," Nachrichten von der Gesellschaft der Wissenschaften zu Göttingen, Mathematisch-Physikalische Klasse, vol. 1919, in collab. with L. Prandtl, pp. 193-217, 1919.

[33] H. Glauert, "L - Airplane Propellers," in Aerodynamic Theory: A General Review of Progress. New York: Dover Publications, 1963, vol. 4, pp. 169-360.

[34] D. M. Pitt and D. A. Peters, "Rotor dynamic inflow derivatives and time constants from various inflow models," presented at the 9th European Rotorcraft Forum, Stresa, Italy: Associazione Italiana di Aeronautica ed Astronautica, Sep. 1983.

[35] R. T. N. Chen, "A survey of nonuniform inflow models for rotorcraft flight dynamics and control applications," Ames Research Center, Moffett Field, California, NASA TM-102219, Nov. 1989.

[36] K. W. Mangler and H. B. Squire, "The induced velocity field of a rotor," Aeronautical Research Council, London, UK, ARC R\&M 2642, 1950.

[37] D. Kunz, "Comprehensive rotorcraft analysis: past, present, and future," presented at the 46th AIAA/ASME/ASCE/AHS/ASC Structures, Structural Dynamics \& Materials Conference, Austin, TX: American Institute of Aeronautics and Astronautics, Apr. 18, 2005. 
[38] D. G. Opoku, D. G. Triantos, F. Nitzsche, and S. G. Voutsinas, "Rotorcraft aerodynamic and aeroacoustic modelling using vortex particle methods," presented at the International Council of the Aeronautical Sciences Congress, Toronto, Canada, 2002.

[39] S. Voutsinas, M. Belessis, and S. Huberson, "Dynamic inflow effects and vortex particle methods," presented at the European Community Wind Energy Conference, Travemünde, Germany, 1993.

[40] K. W. Noonan and G. J. Bingham, "Two-dimensional aerodynamic characteristics of several rotorcraft airfoils at Mach numbers from 0.35 to 0.90," NASA-Langley Research Center, Hampton, Virginia, NASA TM X-73990, 1977.

[41] K. W. Noonan and G. J. Bingham, "Aerodynamic characteristics of three helicopter rotor airfoil sections at Reynolds numbers from model scale to full scale at Mach numbers from 0.35 to 0.90," NASA Langley Research Center, AVRADCOM Research, and Technology Laboratories, Washington, D.C.; Springfield, Va., NASA TP-1701, AVRADCOM TR 80-B-5, 1980.

[42] I. H. Abbott, A. E. Von Doenhoff, and L. Stivers, "Summary of airfoil data," National Advisory Committee for Aeronautics. Langley Aeronautical Lab., Langley Field, VA, NACA TR 824, Jan. 1, 1945.

[43] M. S. Selig, J. J. Gugliemo, A. P. Broeren, and P. Giguere, Summary of Low-Speed Airfoil Data. Virgina Beach, VA: SoarTech Publications, 1995, vol. 1.

[44] M. S. Selig, C. A. Lyon, P. Giguere, C. N. Ninham, and J. J. Gugliemo, Summary of Low-Speed Airfoil Data. Virgina Beach, VA: SoarTech Publications, 1996, vol. 2.

[45] J. D. Anderson, Fundamentals of Aerodynamics, 3rd. Boston: McGraw-Hill, 2001. 
[46] M. U. Tinis, "An algorithm for the aerodynamic analysis of rotating blades using a weak viscous-inviscid interaction method," MASc thesis, Carleton University (Canada), Ottawa, Canada, 2013.

[47] S. K. Roberts and M. I. Yaras, "Modeling transition in separated and attached boundary layers," Journal of Turbomachinery, vol. 127, no. 2, pp. 402-411, Mar. 1, 2004.

[48] M. Drela, "XFOIL: An analysis and design system for low Reynolds number airfoils," in Low Reynolds Number Aerodynamics, ser. Lec. Notes in Eng. 54, Springer-Verlag, 1989.

[49] M. S. Selig, A. Gopalarathnam, P. Giguere, and C. A. Lyon, "Systematic airfoil design studies at low Reynolds numbers," in Fixed and Flapping Wing Aerodynamics for Micro Air Vechicle Applications, ser. Progress in Astronautics and Aeronautics, T. J. Mueller, Ed., vol. 195, American Institute of Aeronautics and Astronautics, 2001, pp. 143-167.

[50] R. Celi, "Recent applications of design optimization to rotorcraft - a survey," presented at the American Helicopter Society 55th Annual Forum, Montreal, Canada, May 1999.

[51] R. Ganguli, "A survey of recent developments in rotorcraft design optimization," Journal of Aircraft, vol. 41, no. 3, pp. 493-510, May 2004.

[52] E. K. P. Chong and S. H. Zak, An Introduction to Optimization, 2nd ed. New York, NY: Wiley, 2001.

[53] J. L. Walsh, "Performance optimization of helicopter rotor blades," NASA Langley Research Center, Hampton, Virginia, NASA TM-104054, Apr. 1991. 
[54] J. L. Walsh, W. J. Lamarsh, and H. M. Adelman, "Fully integrated aerodynamic/dynamic optimization of helicopter rotor blades," presented at the 33rd AIAA, ASME, ASCE, AHS, and ASC Structures,Structural Dynamics and Materials Conference, Dallas, TX, Feb. 1992.

[55] A. Chattopadhyay, T. R. McCarthy, and N. Pagaldipti, "Multilevel decomposition procedure for efficient design optimization of helicopter rotor blades," AIAA Journal, vol. 33, no. 2, pp. 223-230, Feb. 1995.

[56] A. Le Pape and P. Beaumier, "Numerical optimization of helicopter rotor aerodynamic performance in hover," Aerospace Science and Technology, vol. 9, no. 3, pp. 191-201, 2005.

[57] MathWorks. Optimization toolbox, R2014b Documentation, [Online]. Available: http://www. mathworks.com/help/optim/index.html (Accessed Dec. 4, 2014).

[58] UIUC airfoil coordinate database, UIUC Applied Aerodynamics Group, [Online]. Available: http : / / aerospace . illinois . edu /m-selig/ads / coord_ database.html (Accessed Jun. 25, 2014).

[59] S. C. Chapra, Applied numerical methods with MATLAB for engineers and scientists, 2nd. Boston, MA: McGraw-Hill Higher Education, 2008.

[60] W. A. Timmer and C. Bak, "Aerodynamic characteristics of wind turbine blade airfoils," in Advances in Wind Turbine Blade Design and Materials, P. Brøndsted and R. Nijssen, Eds., Cambridge, UK: Woodhead Publishing, 2013, pp. 109-149.

[61] C. Bak, H. Aagaard Madsen, M. Gaunaa, U. Schmidt Paulsen, P. Fuglsang, J. Romblad, N. A. Olesen, P. Enevoldsen, J. Laursen, and L. Jensen, "DAN-AERO 
MW: Comparisons of airfoil characteristics for two airfoils tested in three different wind tunnels," presented at the Torque 2010, Crete, Greece: European Wind Energy Association (EWEA), 2010, pp. 59-70.

[62] P. A. Krogstad, J. A. Karlsen, and M. S. Adaramola, "Performance of a model wind turbine," presented at the 17th Australasian Fluid Mechanics Conference, Auckland, New Zealand: University of Auckland, 2010, pp. 112-115.

[63] L. U. Dadone, "US army helicopter design datcom. Volume 1: Airfoils," Ames Research Center, Moffett Field, California, USAAMRDL CR76-2, 1976.

[64] C. Kaplan, "Effect of compressibility at high subsonic velocities on the lifting force acting on an elliptic cylinder," Langley Aeronatical Laboratory, Langley Field, VA, NACA-TR-834, 1946.

[65] R. Harrington, "Full-scace-tunnel investigation of the static thrust performance of a coaxial helicopter rotor.," Langley Aeronatical Laboratory, Washington, DC, NACA TN 2318, 1951.

[66] E. N. Jacobs and A. Sherman, "Airfoil section characteristics as affected by variations of the Reynolds number," National Advisory Committee for Aeronautics, Langley Field, VA, NACA-TR-586, 1937.

[67] F. D. Harris, "Articulated rotor blade flapping motion at low advance ratio," Journal of the American Helicopter Society, vol. 17, no. 1, pp. 41-48, 1972.

[68] MathWorks. fmincon, R2014b Documentation, [Online]. Available: http:// www . mathworks. com/help/optim/ug/fmincon. html (Accessed Dec. 1, 2014).

[69] J. H. Ginsberg, Advanced Engineering Dynamics, 2nd ed. Cambridge, UK: Cambridge University Press, 1998. 


\section{Appendix A}

\section{Airfoil Characteristics Prediction Validation}

This appendix contains figures of airfoil performance coefficients generated using VII [46] and XFOIL [48]. Airfoils for which experimental wind-tunnel data was available in the form of References [17], [18] and [19] were used to assess the applicability of the two numerical packages to the incompressible low Reynolds number regime. VII lift polars were corrected using the lift stall estimation model described in Section 3.1.1.

Nine airfoils were chosen each from Reference [17] at Reynolds numbers from 100, 000 to 500, 000 and from References [18] and [19] at Reynolds numbers from 700,000 to $1,500,000^{1}$. Each selection of airfoils consists of three ranges of thickness and three different amounts of camber in each thickness group. The experimental data mostly corresponds to clean or "un-tripped" airfoil test configurations. The XFOIL results were generated assuming incompressible conditions and $e^{n}$ transition parameter $n_{\text {crit }}=9$.

\footnotetext{
${ }^{1}$ Note that References [18] and [19] did not include section pitching moment data at all Reynolds numbers at which lift and drag data was provided.
} 

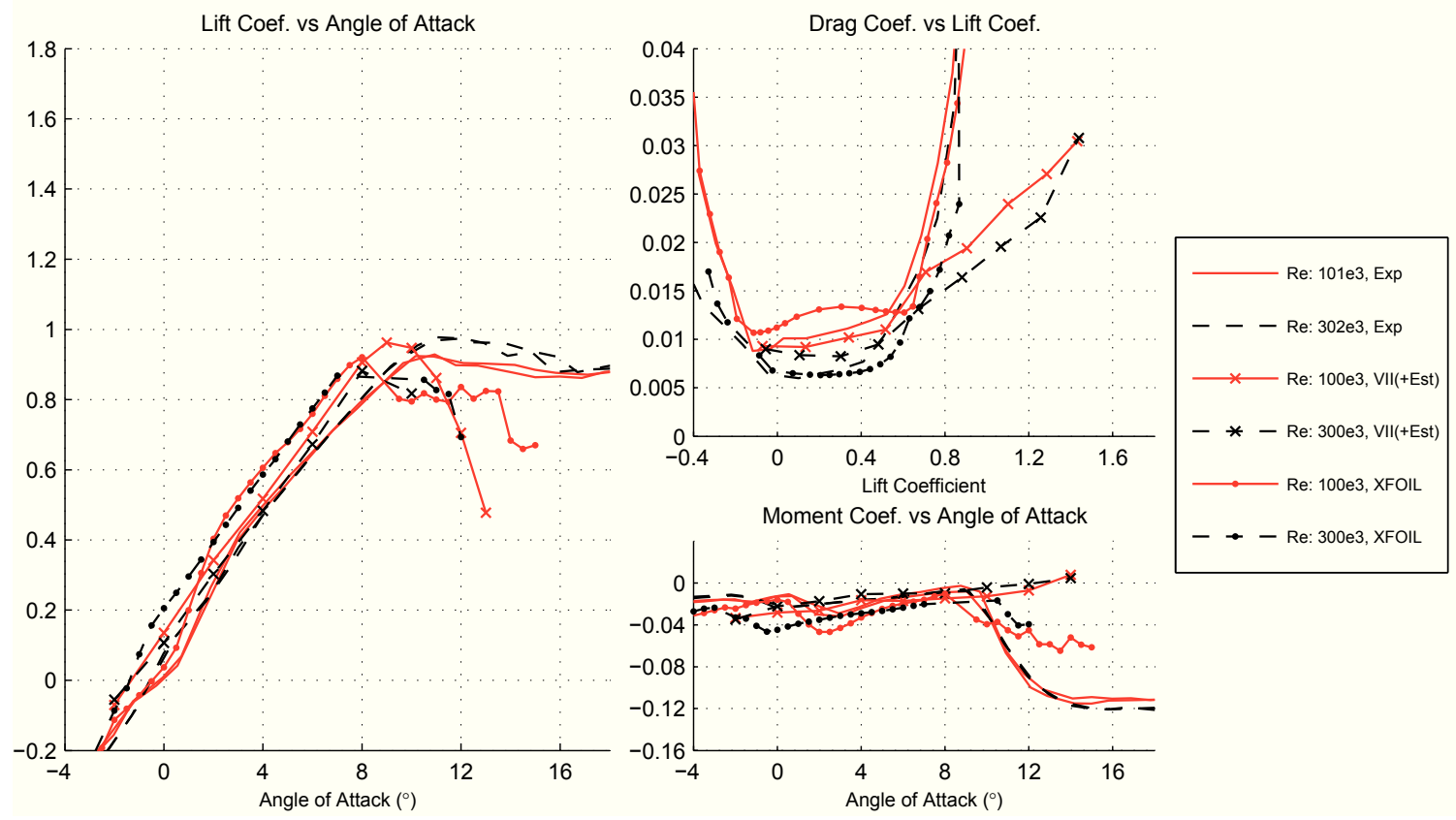

(a) Airfoil: Selig S6063, ${ }^{t} / \mathrm{c}: 7.0 \%,{ }^{\mathrm{cam}} / \mathrm{c}: 1.4 \%[17]$.
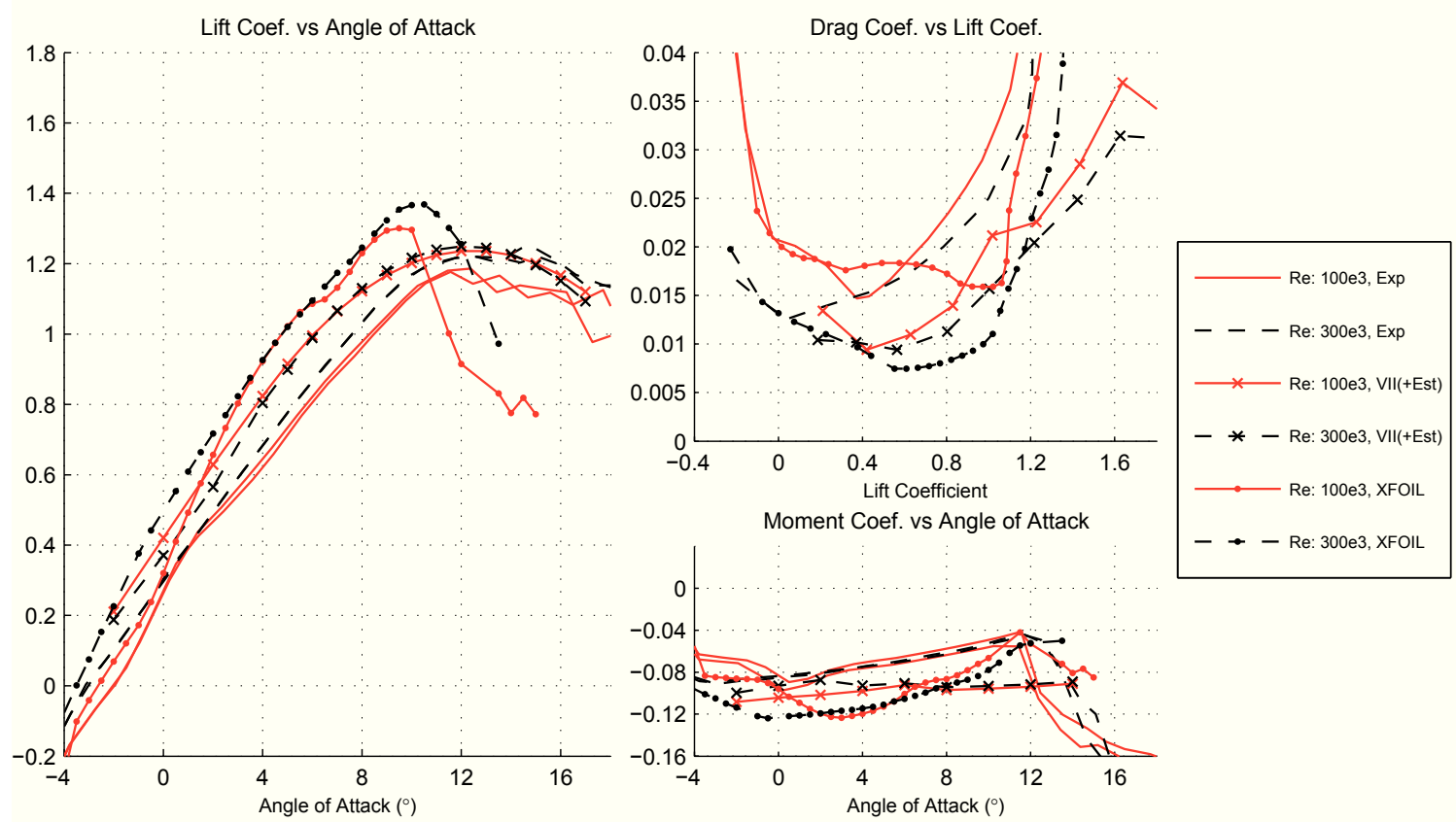

(b) Airfoil: Archer A18, ${ }^{t} / \mathrm{:}: 7.3 \%,{ }^{\mathrm{cam}} / \mathrm{c}: 3.8 \%[17]$.

Figure A.1: Comparison of two-dimensional airfoil property data from VII [46] including the stall estimation model and XFOIL [48] with respect to experimental data from the University of Illinois at Urbana-Champaign [17]. 

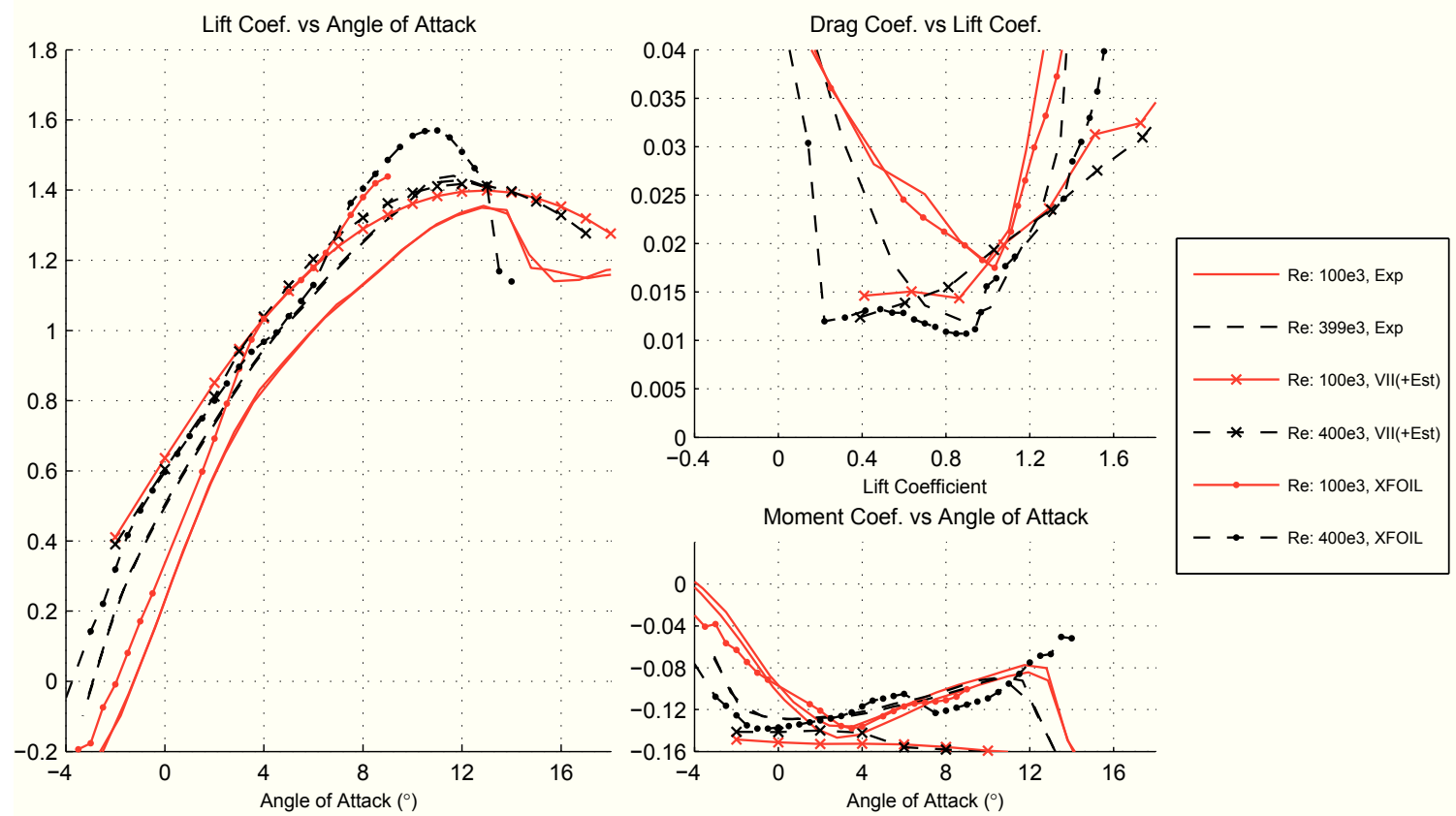

(c) Airfoil: Bergey BW-3, ${ }^{t} / c: 5.0 \%,{ }^{\mathrm{cam}} / \mathrm{c}: 5.8 \%$ [17].
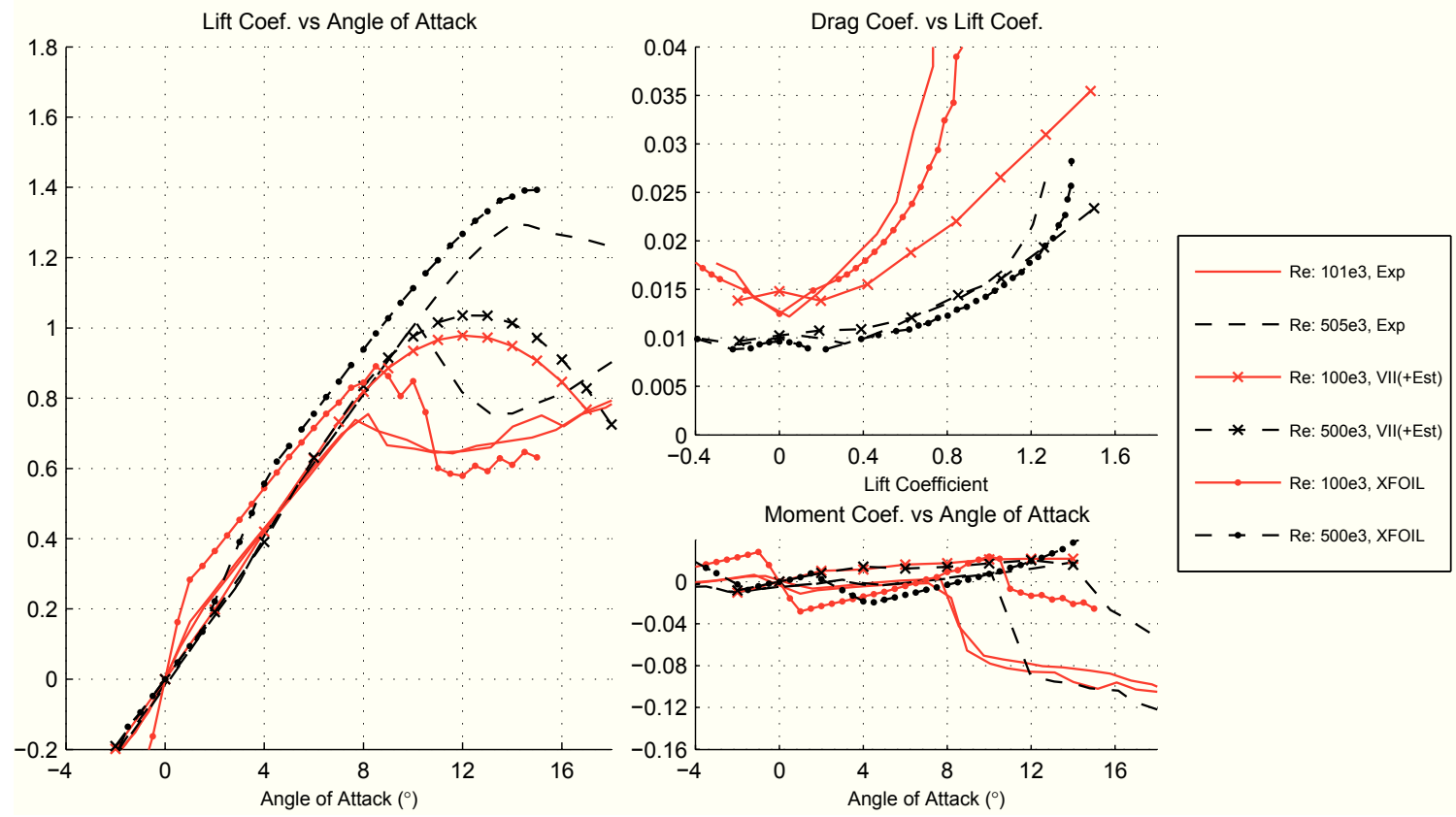

(d) Airfoil: Eppler E472, ${ }^{t} / \mathrm{c}: 12.1 \%,{ }^{\mathrm{cam}} / \mathrm{c}: 0 \%$ [17].

Figure A.1: Continued. 


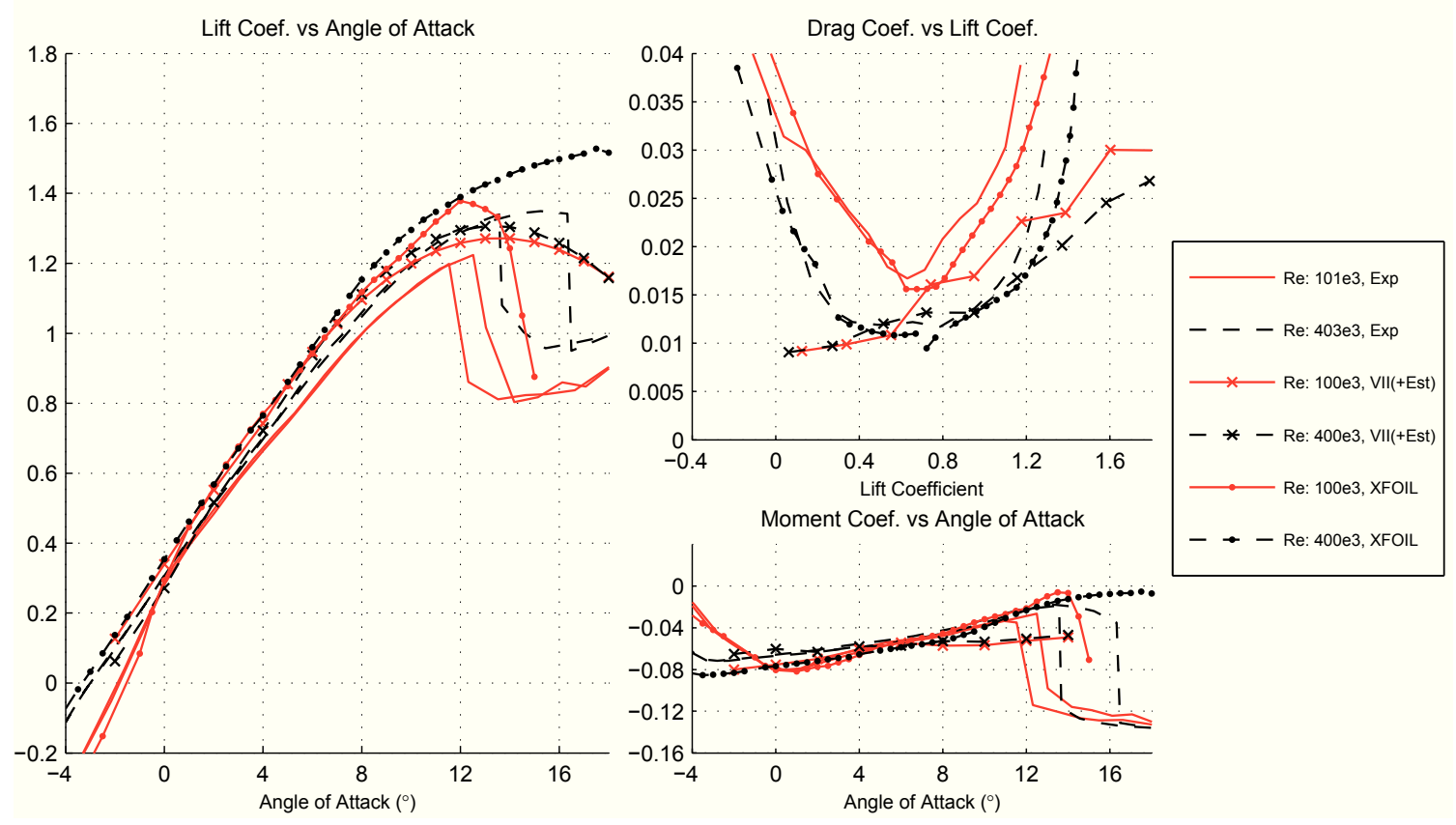

(e) Airfoil: Great Planes PT-40, ${ }^{t} / c: 11.6 \%,{ }^{\mathrm{cam}} / \mathrm{c}: 2.9 \%$ [17].
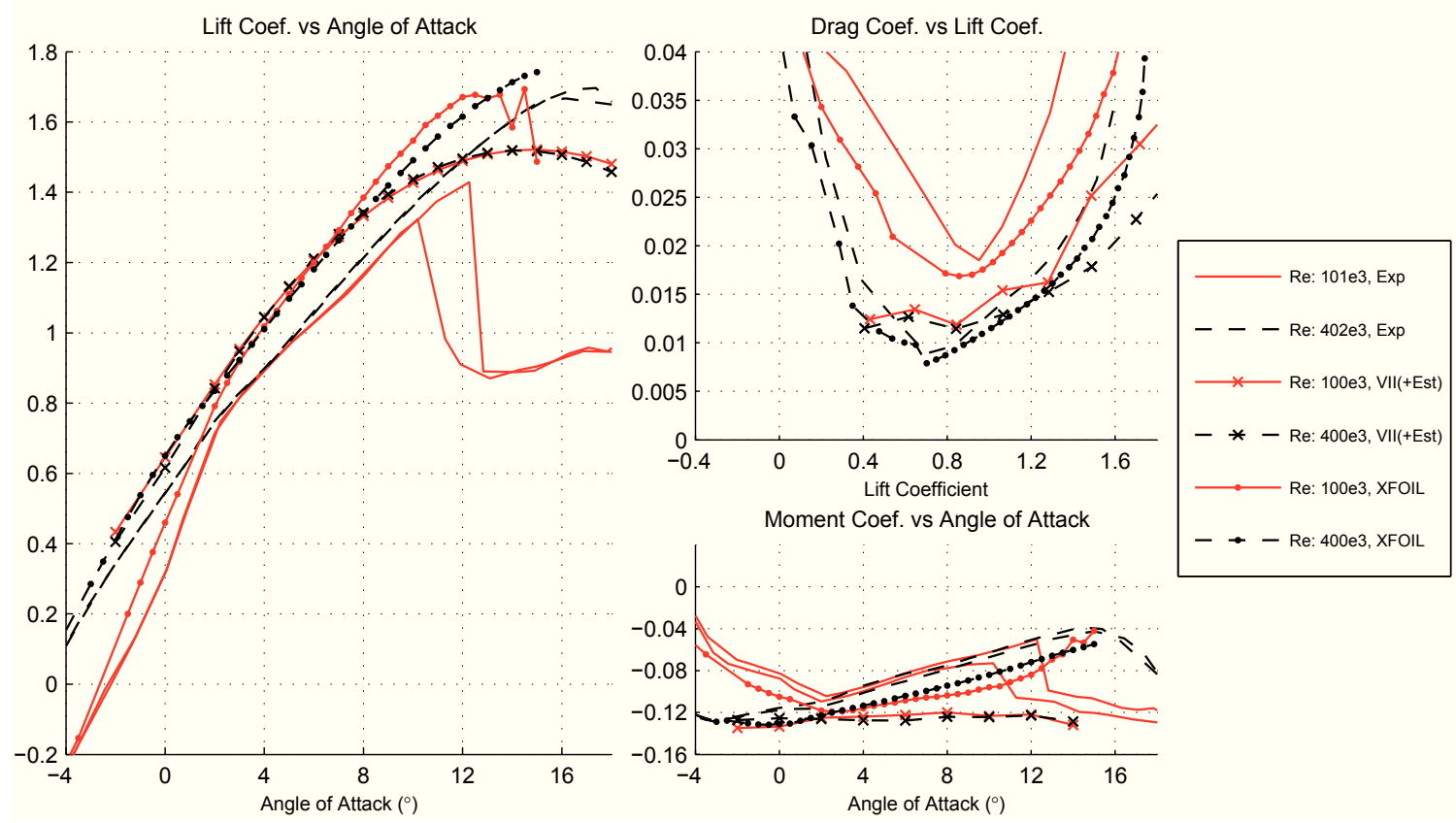

(f) Airfoil: Jacobs USNPS4, ${ }^{t} / \mathrm{c}: 11.9 \%,{ }^{\mathrm{cam}} / \mathrm{c}: 9.0 \%$ [17].

Figure A.1: Continued. 

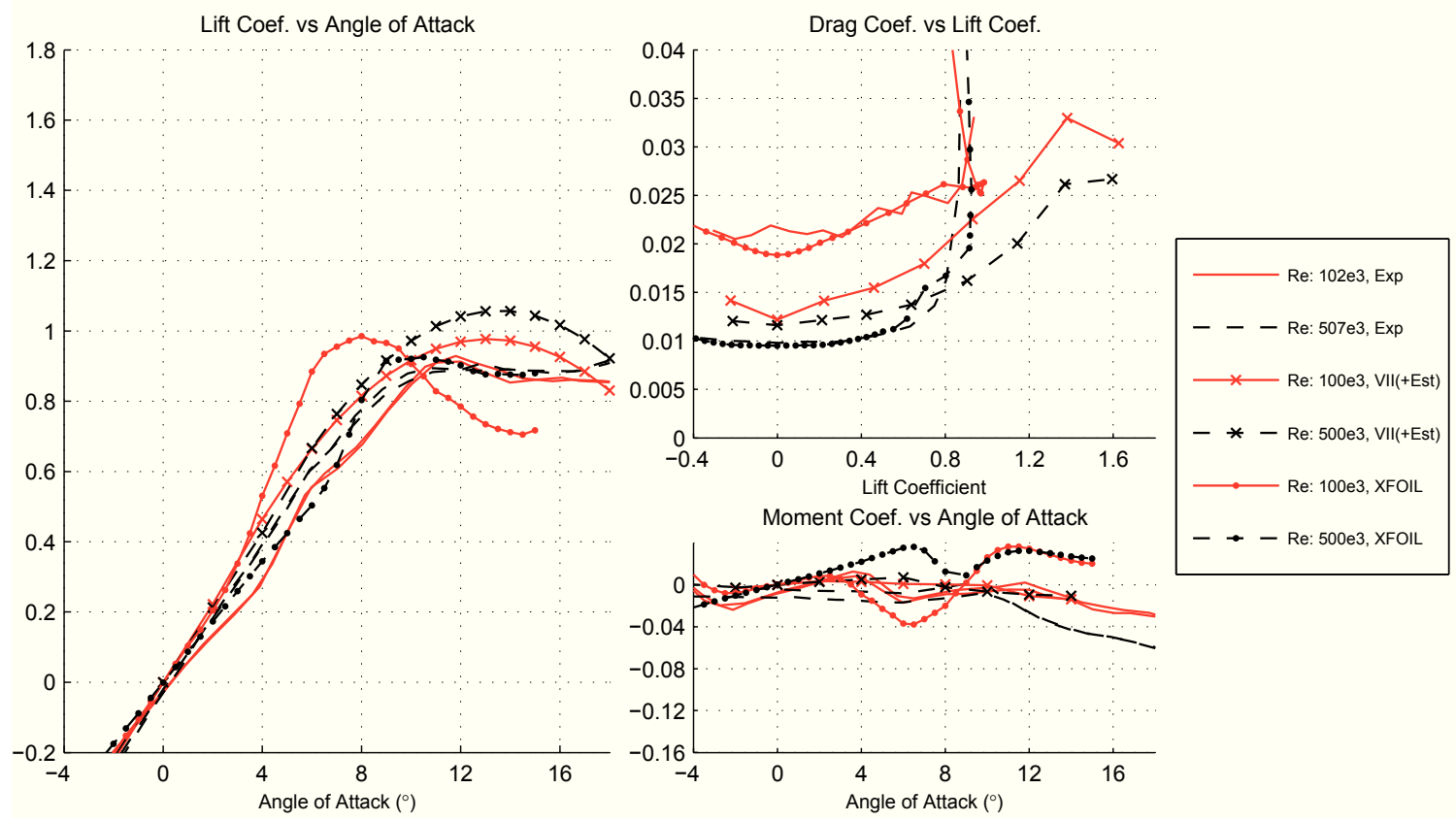

(g) Airfoil: Great Planes R/C Ultra-Sport 1000, ${ }^{t} / \mathrm{c}: 18.6 \%,{ }^{\mathrm{cam}} / \mathrm{c}: 0 \%$ [17].
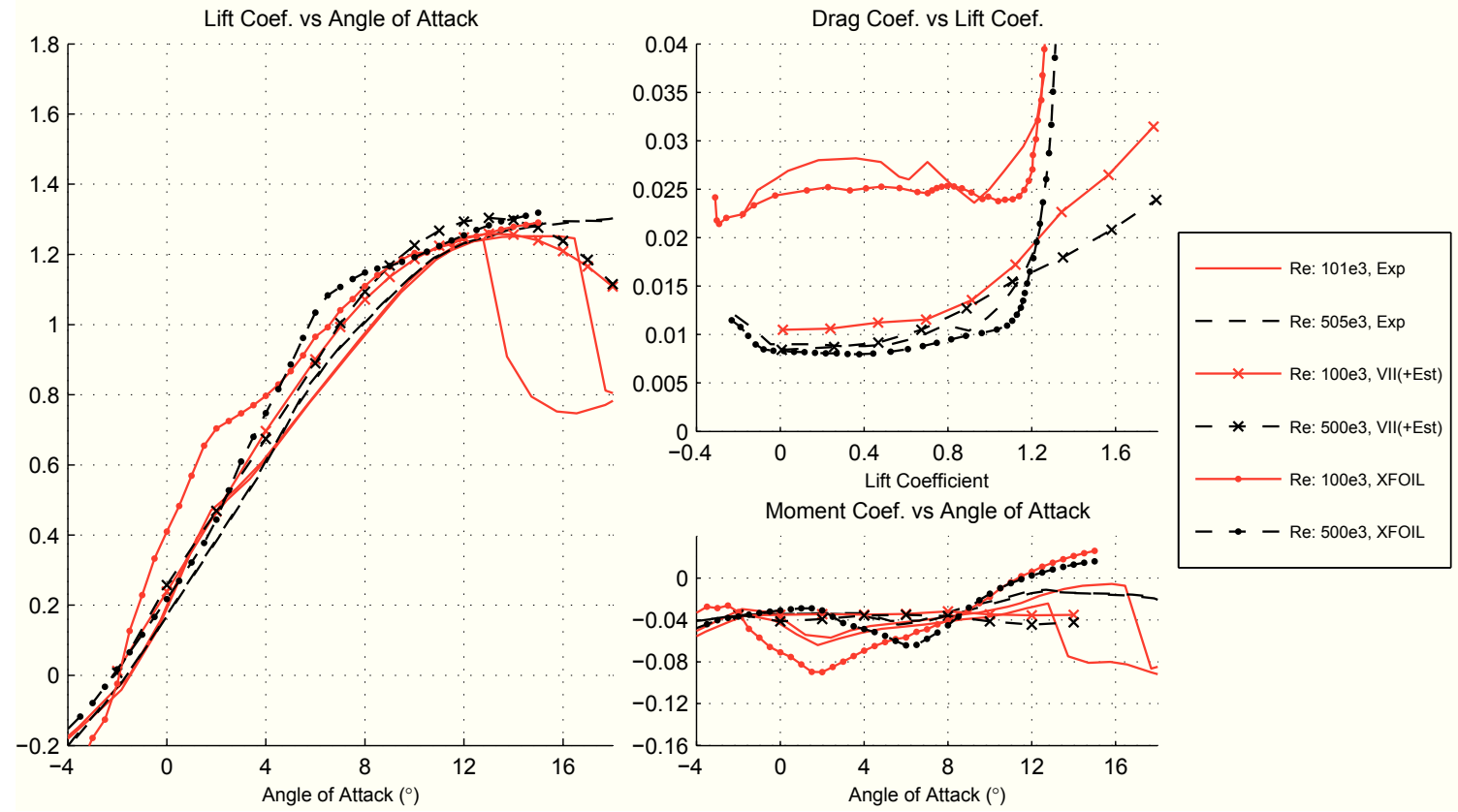

(h) Airfoil: Selig S8037, ${ }^{t} / \mathrm{c}: 16.0 \%,{ }^{\mathrm{cam}} / \mathrm{c}: 2.6 \%$ [17].

Figure A.1: Continued. 


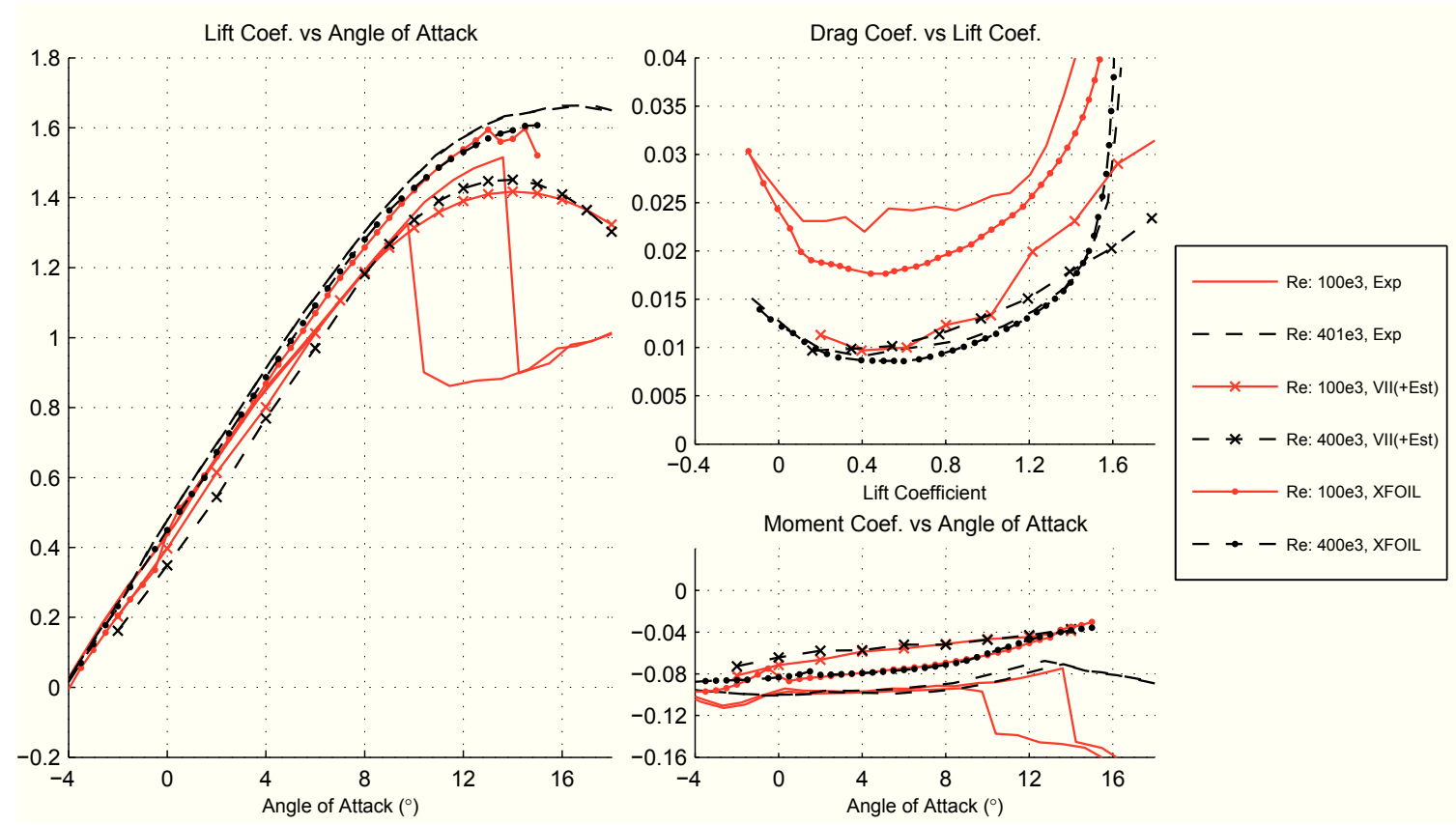

(i) Airfoil: Selig/Donovan SD7062, ${ }^{t} / c: 14.0 \%,{ }^{\mathrm{cam}} / \mathrm{c}: 4.0 \%$ [17].

Figure A.1: Continued. 

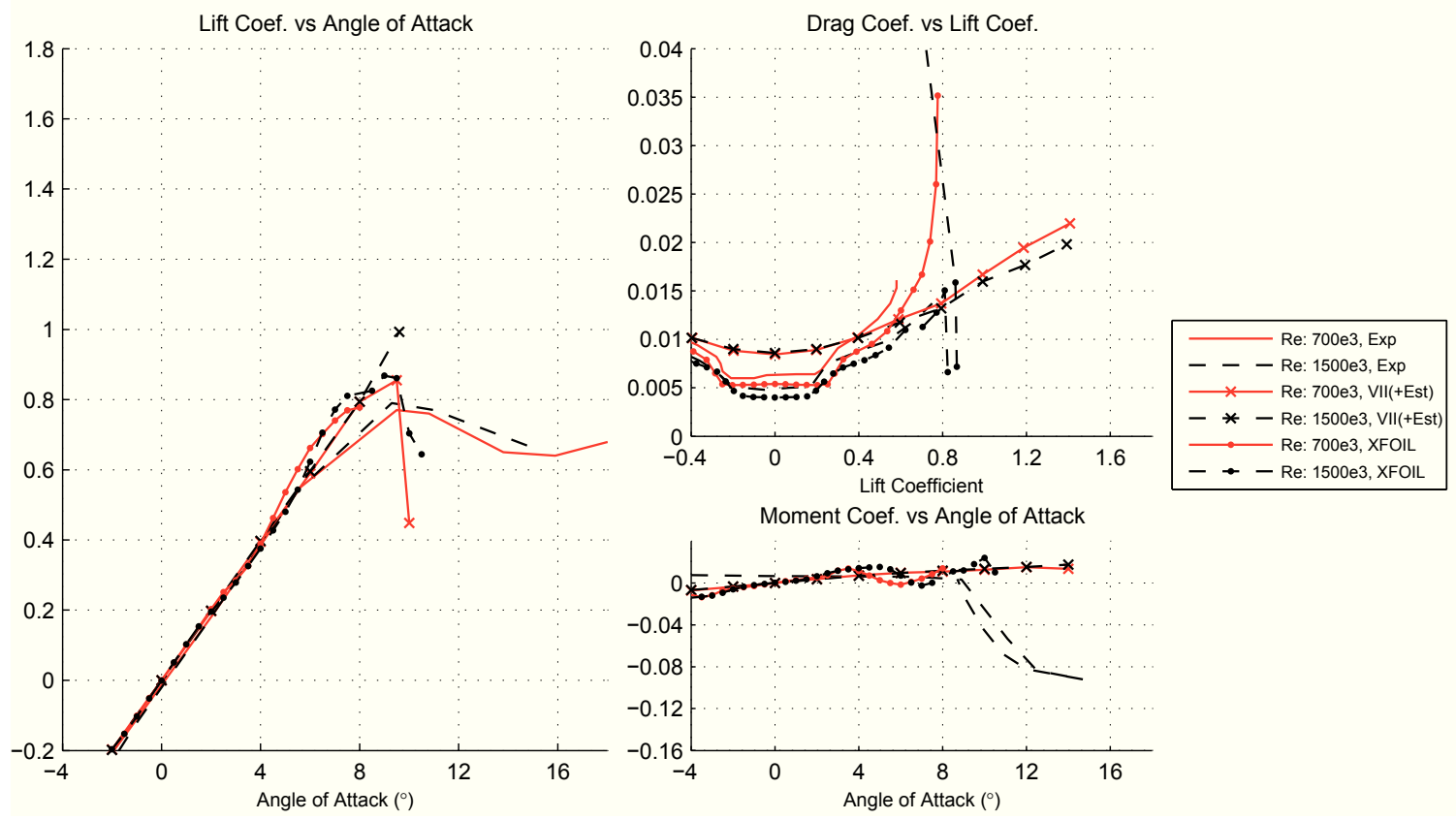

(a) Airfoil: LWK 80-080, ${ }^{t} / \mathrm{c}: 8.1 \%,{ }^{\mathrm{cam}} / \mathrm{c}$ : 0\% [19].
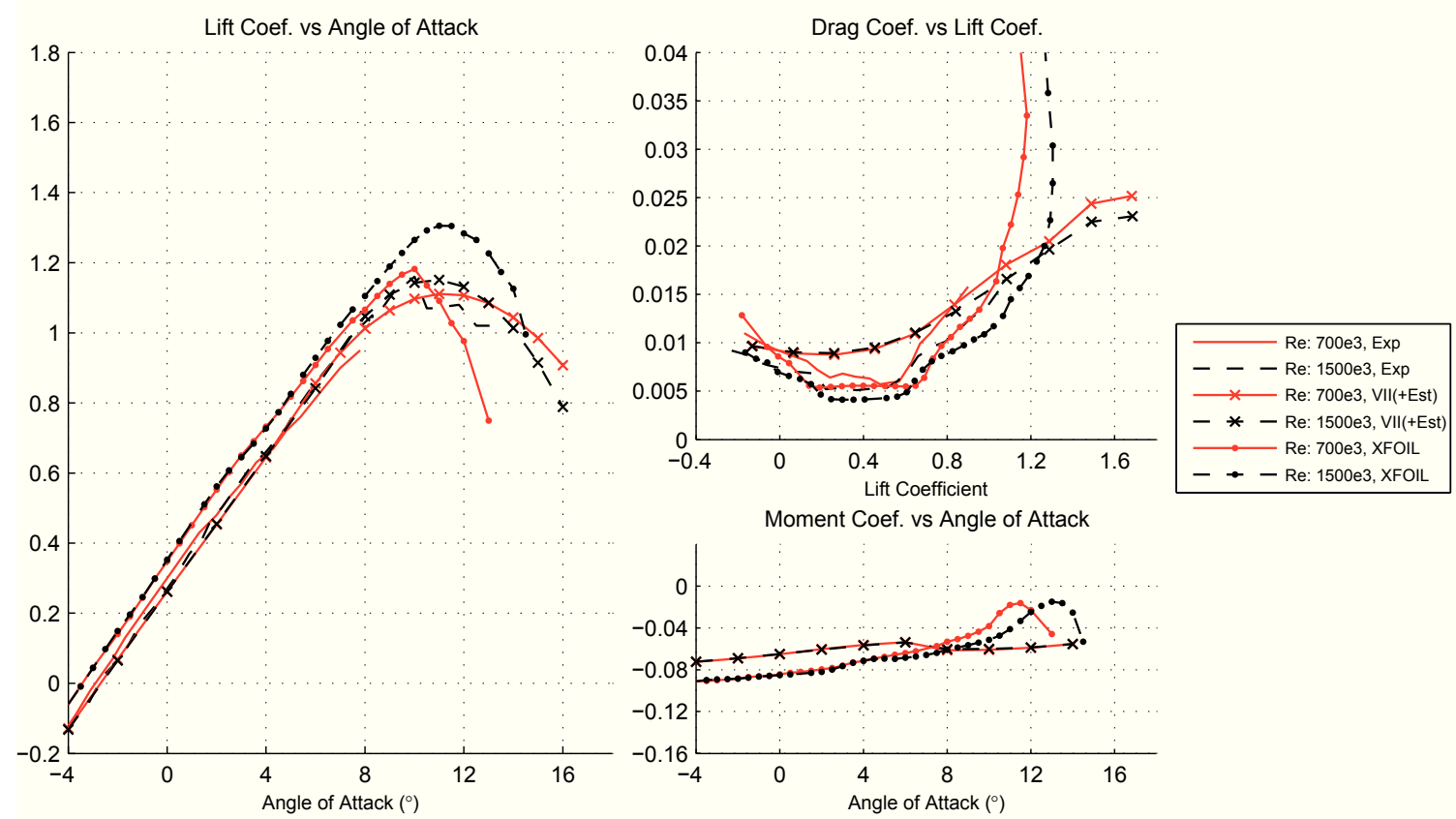

(b) Airfoil: Wortmann FX 80-080, ${ }^{t} / \mathrm{c}: 8.1 \%,{ }^{\mathrm{cam}} / \mathrm{c}: 2.2 \%$ [19].

Figure A.2: Comparison of two-dimensional airfoil property data from VII [46] including the stall estimation model and XFOIL [48] with respect to experimental data from Universität Stuttgart [18], [19]. 

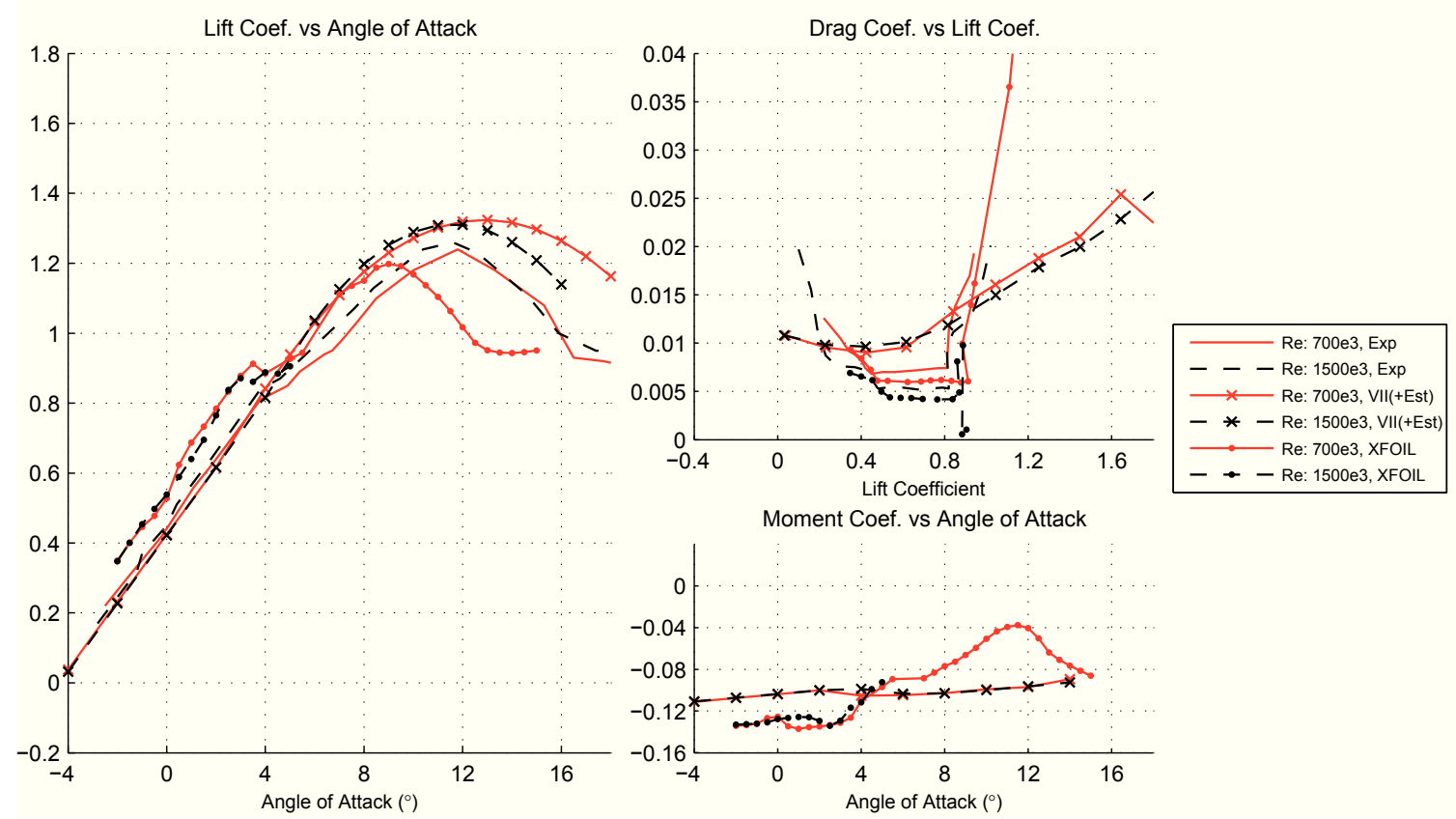

(c) Airfoil: Wortmann FX 74-080, ${ }^{t} / \mathrm{c}: 8.2 \%,{ }^{\mathrm{cam}} / \mathrm{c}: 4.3 \%$ [19].
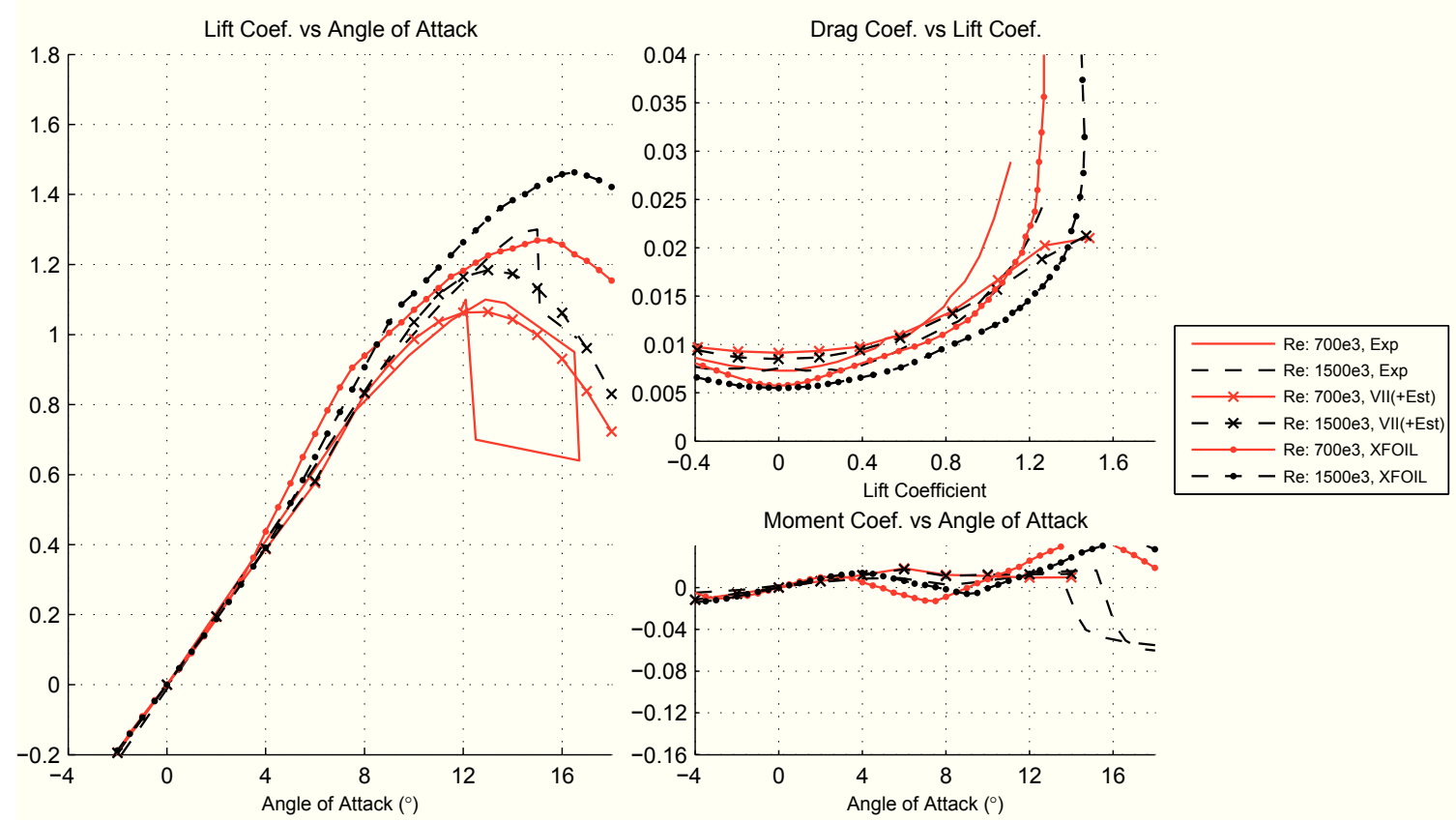

(d) Airfoil: Wortmann FX 76-120, ${ }^{t} / \mathrm{c}: 12.1 \%,{ }^{\mathrm{cam}} / \mathrm{c}_{\mathrm{c}}$ : 0\% [19].

Figure A.2: Continued. 

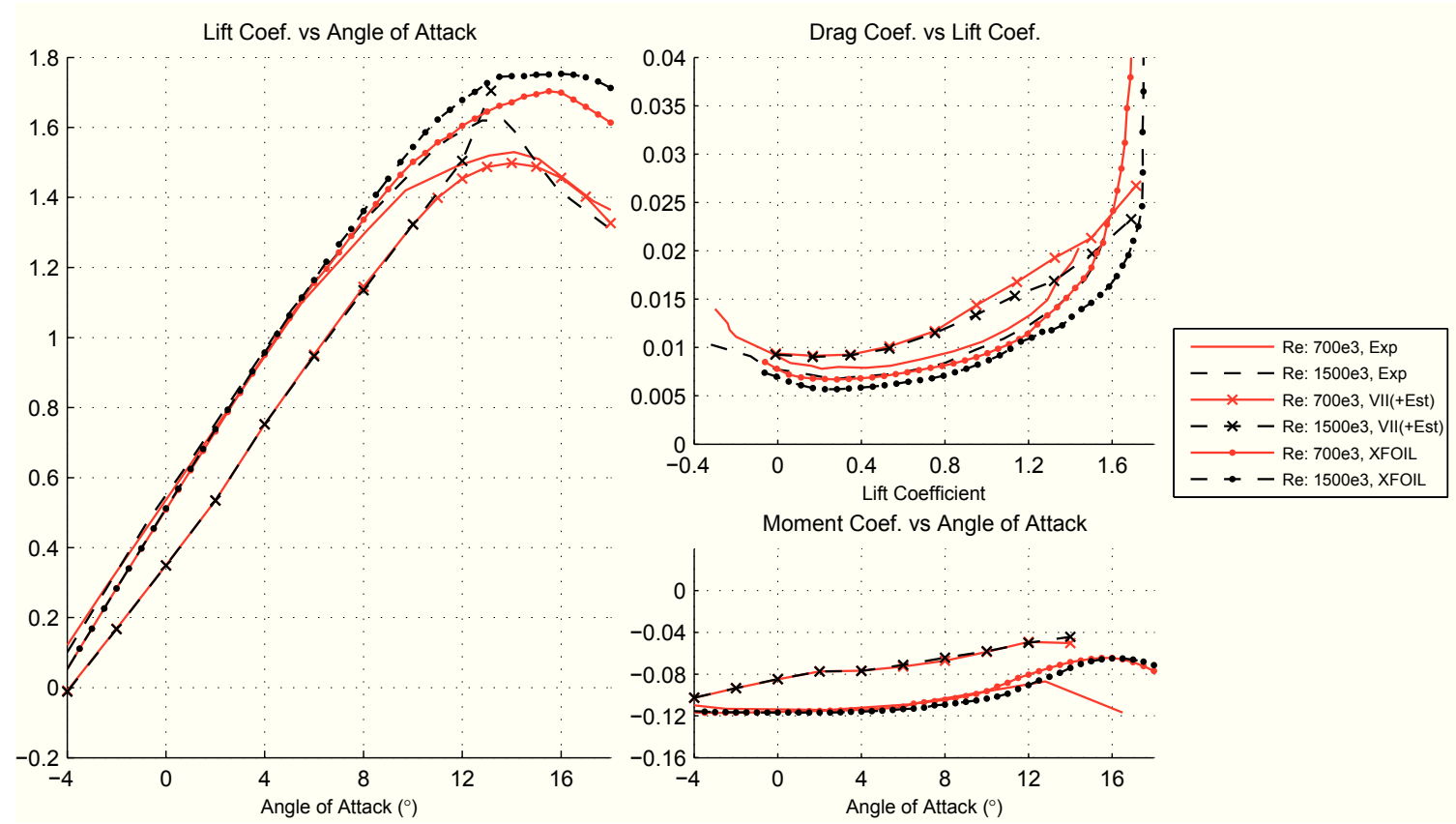

(e) Airfoil: Wortmann FX 60-126, ${ }^{t} / c: 12.6 \%,{ }^{c a m} / c: 3.5 \%$ [18].
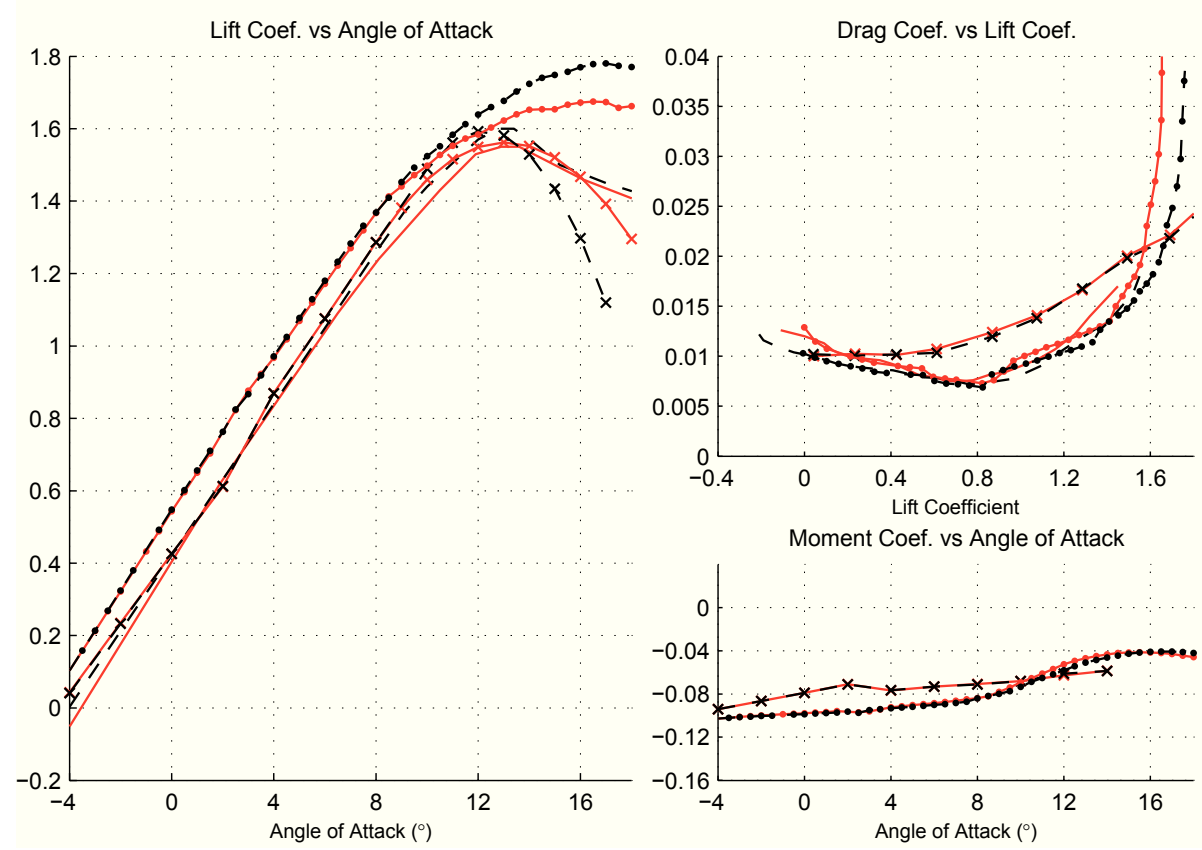

(f) Airfoil: Göttingen $532,{ }^{t} / c: 12.5 \%,{ }^{\mathrm{cam}} / \mathrm{c}: 4.8 \%$ [18].

Figure A.2: Continued. 

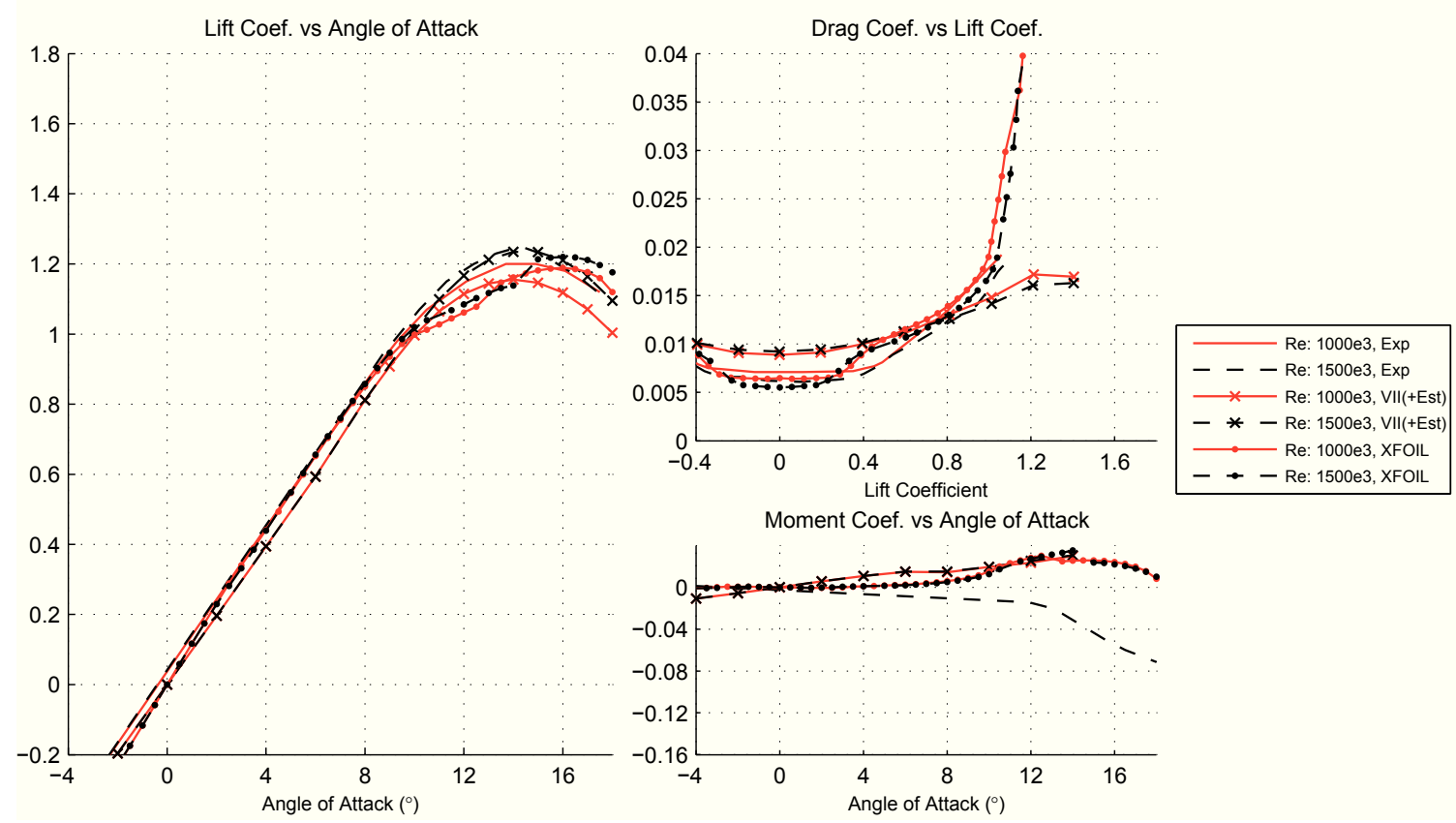

(g) Airfoil: Wortmann FX 71-L-150/20, ${ }^{\mathrm{t}} / \mathrm{c}: 15.0 \%,{ }^{\mathrm{cam}} / \mathrm{c}: 0 \%$ [18].
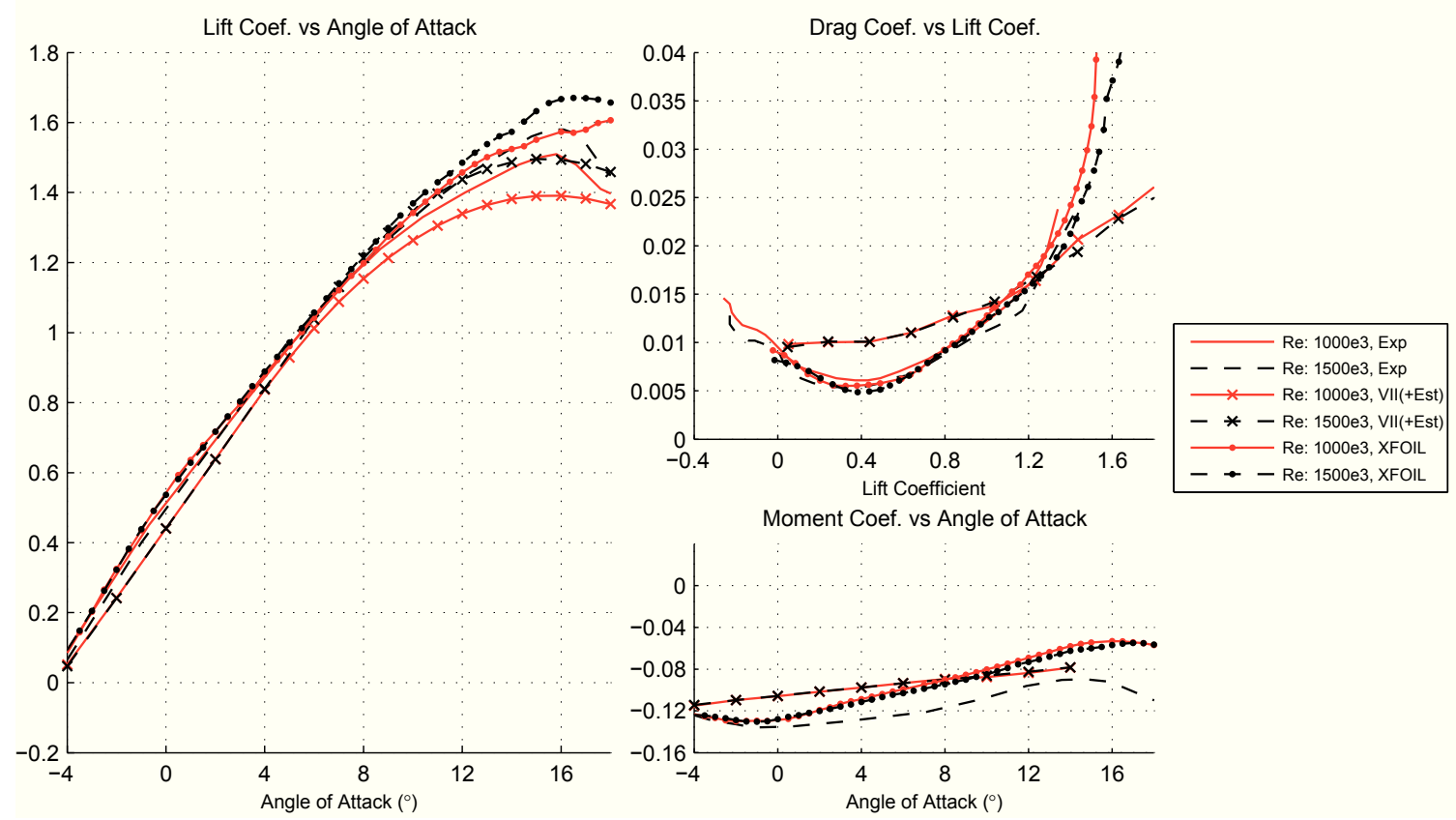

(h) Airfoil: Wortmann FX 60-157, ${ }^{t} / \mathrm{c}: 15.7 \%,{ }^{\mathrm{cam}} / \mathrm{c}: 2.7 \%[18]$.

Figure A.2: Continued. 

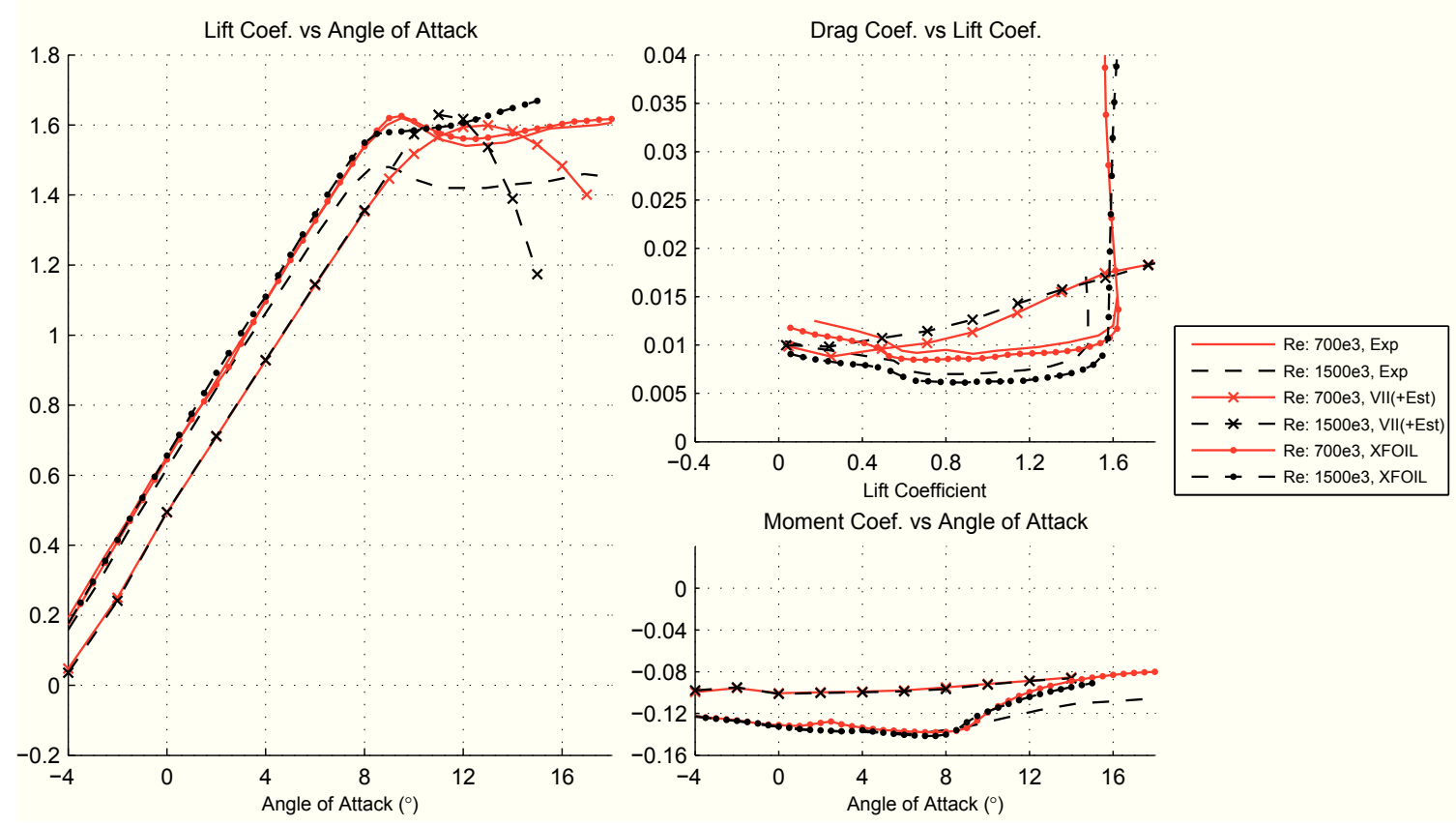

(i) Airfoil: Althaus AH 95-160, ${ }^{t} / c: 16.0 \%,{ }^{\mathrm{cam}} / \mathrm{c}: 5.5 \%$ [19].

Figure A.2: Continued. 


\section{Appendix B}

\section{Additional Hover Module Validation Figures}

\section{and Discussion}

In this appendix the output of the hover module is compared to three experimental scaled rotor studies from References [21], [22] and [23] and a full scale rotor study presented in Reference [65]. General observations of underpredicted power coefficient and overpredicted figure of merit have already been discussed in Section 3.2.2. The discussion here will focus on the relation of figure of merit to thrust coefficient with respect to specific test rotor characteristics.

All simulated results were obtained using XFOIL generated airfoil coefficients. Important rotor parameters are summarized in tabular form. Further test specific details can be obtained from the respective source documents. 
Reference [21] compares the performance of three scaled test rotors at multiple tip speeds. The baseline rotor was based on the Sikorsky UH-60A rotor and had a rectangular planform with a tip swept $20^{\circ}$ aft for the outer $7.5 \%$ of the blade span. Compared to this baseline were two rotors of equivalent thrust weighted solidity, denoted TR3 and TR5, with 3:1 and 5:1 linear planform taper applied to the outer $20 \%$ of the rotor span, respectively. TR3 and TR5 did not include any sweep. While all three blade sets had $-16^{\circ}$ blade twist, the rate of twist on the baseline blades was not constant along the span. Figure B.1 shows the experimental and simulated results at tip speeds of $91.4 \mathrm{~m} / \mathrm{s}(300 \mathrm{ft} / \mathrm{s})$ and $182.9 \mathrm{~m} / \mathrm{s}(600 \mathrm{ft} / \mathrm{s})$.

At $91.4 \mathrm{~m} / \mathrm{s}$ rotor TR5 showed highest figure of merit while at $182.9 \mathrm{~m} / \mathrm{s}$ best performance was observed for rotor TR3. A significant performance difference between the different rotors was only observed near the upper end of the thrust coefficient range. At
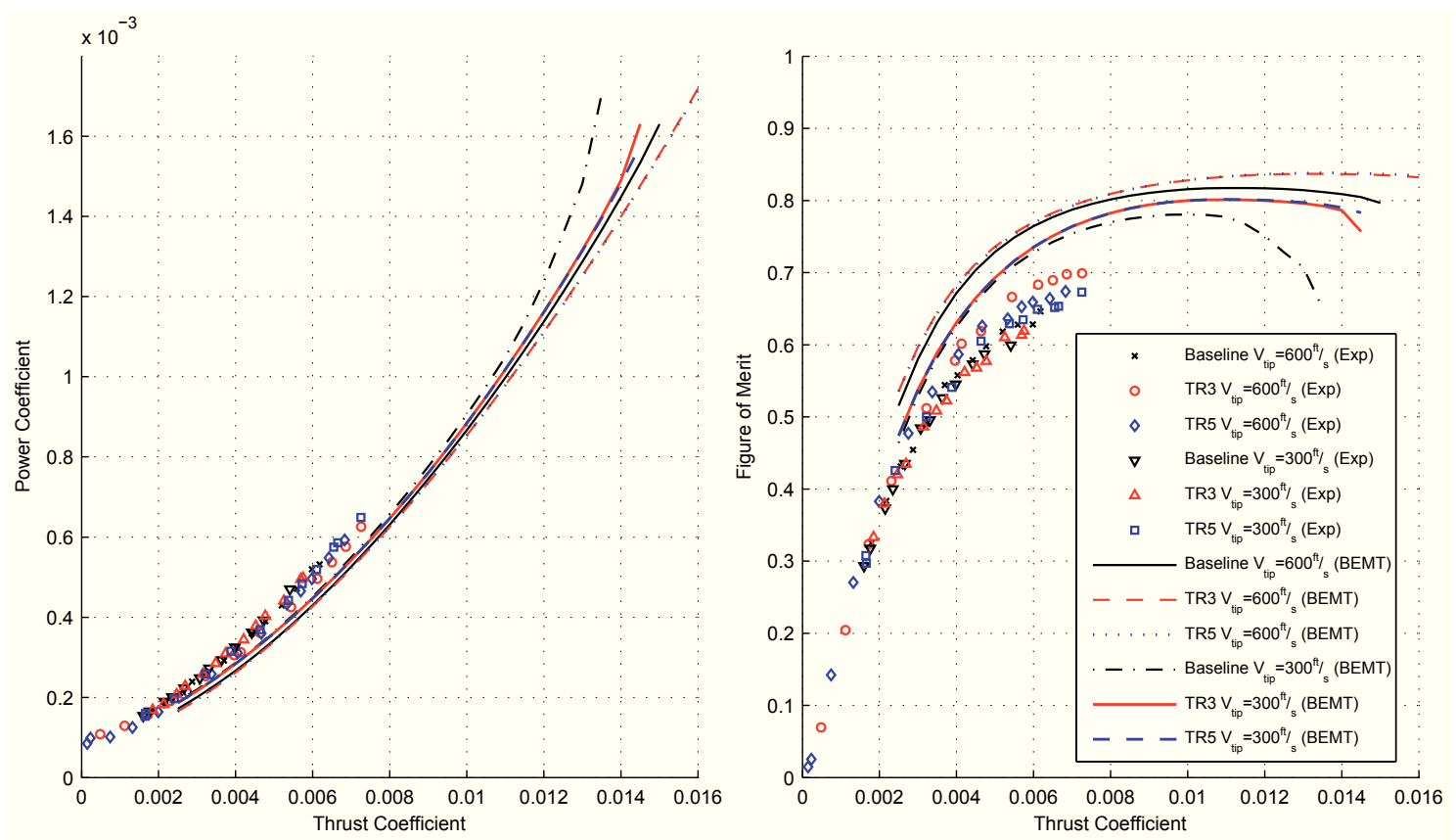

Figure B.1: Power coefficient and figure of merit simulated using the hover module compared to experimental results for rotors with varying taper ratios [21]. 
both speeds the baseline rotor performed weakest, though at $91.4 \mathrm{~m} / \mathrm{s}$ the performance difference between the baseline rotor and TR3 was small. Comparing the two velocities, the observed figure of merit increased by approximately $3 \%$ for the baseline rotor and TR5, while TR3 saw a larger increase on the order or $8 \%$. The experimental thrust coefficient range was power limited and therefore did not reach observable peaks in figure of merit.

The results of the hover module reflect the performance advantage of the tapered TR3 and TR5 rotors over the baseline rotor. However, the predicted performance difference between TR3 and TR5 was small with TR5 showing marginally higher figure of merit at both simulated velocities. The figure of merit increase between the 91.4 and $182.9 \mathrm{~m} / \mathrm{s}$ cases was simulated at approximately $3-4 \%$ for all three rotors. At the lower speed the simulated rotor figure of merit dropped off at significantly lower thrust coefficients.

Table B.1: Test condition and rotor characteristics: variation of taper ratio [21].

\begin{tabular}{cccc}
\hline Parameter & Baseline & TR3 & TR5 \\
\hline \hline Number of Blades & 4 & 4 & 4 \\
Diameter $(\mathrm{m})$ & 2.03 & 2.03 & 2.03 \\
Rotorspeed $(\mathrm{rpm})$ & $859.4,1719$ & $859.4,1719$ & $859.4,1719$ \\
Tip Speed $(\mathrm{m} / \mathrm{s}$ ) & $91.4,182.9$ & $91.4,182.9$ & $91.4,182.9$ \\
Solidity & 0.0825 & 0.0825 & 0.0825 \\
Twist $\left({ }^{\circ}\right)$ & -16 (non-linear) & -16 & -16 \\
Taper & None & $3: 1 @ 0.8 R$ & $5: 1 @ 0.8 R$ \\
Airfoil & NACA 0012 & NACA 0012 & NACA 0012 \\
Notes & Swept Tip & - & - \\
& (not simulated) & & \\
\hline
\end{tabular}


The comparison of the hover module to Reference [22] has already been presented in Section 3.2.2. In this study two test rotors with different airfoils were compared at three tip speeds. The baseline rotor airfoil was the NACA 0012 and the second rotor featured the US Army/NASA RC(4)-10, RC(3)-10 and RC(3)-08 airfoils. Figure B.2 displays results at tip speeds of $172.8 \mathrm{~m} / \mathrm{s}(567 \mathrm{ft} / \mathrm{s})$ and $216.1 \mathrm{~m} / \mathrm{s}(709 \mathrm{ft} / \mathrm{s})$.

The experimental results show an approximately $3 \%$ maximum figure of merit advantage for the "advanced" airfoil selection of the second test rotor over the baseline result at the lower tip speed. At the higher tip speed the difference in maximum figure of merit was similar, but performance drops off at slightly lower thrust coefficients. At both speeds the difference in figure of merit between the baseline rotor and the rotor with the advanced airfoil selection was greatest in the upper thrust coefficient range, where the peaks in figure merit occurred at higher thrust coefficients of 0.009 and 0.008
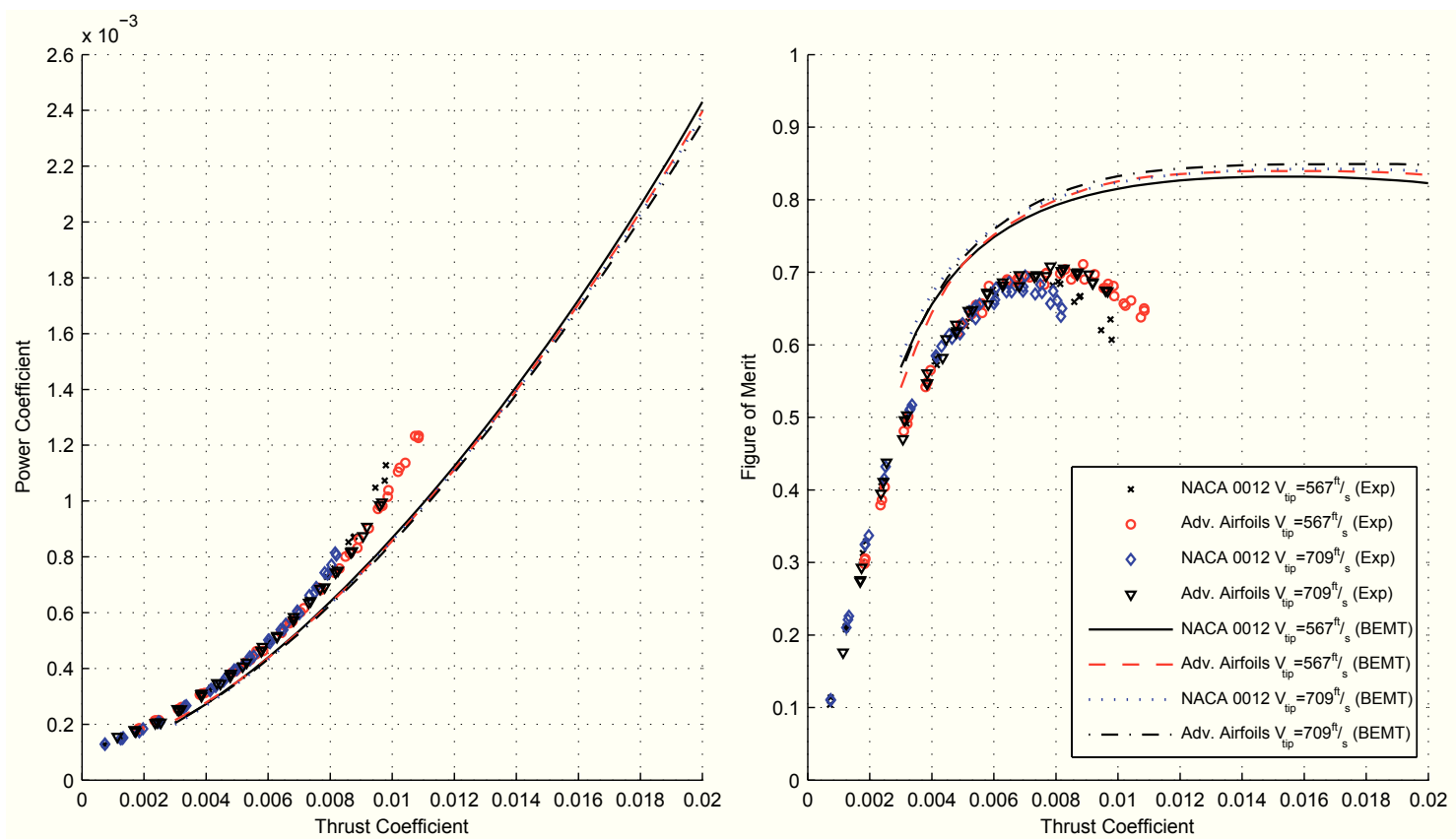

Figure B.2: Power coefficient and figure of merit simulated using the hover module compared to experimental results for rotors with different airfoils [22]. 
for the advanced rotor compare to 0.008 and 0.007 for the baseline rotor.

The hover module predicted results show a small performance gain on the order of $1 \%$ figure of merit from the use of the advances airfoils at thrust coefficients above 0.005. The increased tip speed resulted in a slight increase in figure of merit. The peaks in figure of merit are very flat at thrust coefficients near $0.015-0.016$.

Table B.2: Test condition and rotor characteristics: advanced airfoil selection [22].

\begin{tabular}{ccc}
\hline Parameter & Baseline & Advanced \\
\hline \hline Number of Blades & 4 & 4 \\
Diameter $(\mathrm{m})$ & 1.65 & 1.65 \\
Rotorspeed $(\mathrm{rpm})$ & 2000,2500 & 2000,2500 \\
Tip Speed $(\mathrm{m} / \mathrm{s})$ & $172.8,216.1$ & $172.8,216.1$ \\
Solidity & 0.0977 & 0.0977 \\
Twist $\left(^{\circ}\right)$ & -13 & -13 \\
Taper & $3: 1 @ 0.75 R$ & $3: 1 @ 0.75 R$ \\
Airfoils & NACA 0012 & $\mathrm{RC}(4)-10, \mathrm{RC}(3)-10$ \\
& & $\& \mathrm{RC}(3)-08$ \\
\hline
\end{tabular}


In Reference [23] four test rotors were compared. The baseline rotor had a rectangular planform and the other three rotors featured 3:1 linear taper applied to the outer portion of the blade starting at $94 \%, 75 \%$ and $50 \%$ radius, respectively.

As shown in Figure B.3, best performance with a maximum figure of merit value of approximately $74 \%$ was observed for the rotor featuring taper initialization at $94 \%$ radius. The other two tapered rotors showed only marginal differences in performance compared to the baseline rotor. Maximum figure of merit values for the baseline rotor and the two rotors with taper starting at 50\% and 75\% were measured at 72,71 and $72 \%$, respectively. All test rotors attained their peak figure of merit values near a thrust coefficient of 0.008 .

The hover module predicted results show a trend of increasing figure of merit with respect to decreasing radial location of taper initialization. The thrust coefficient at
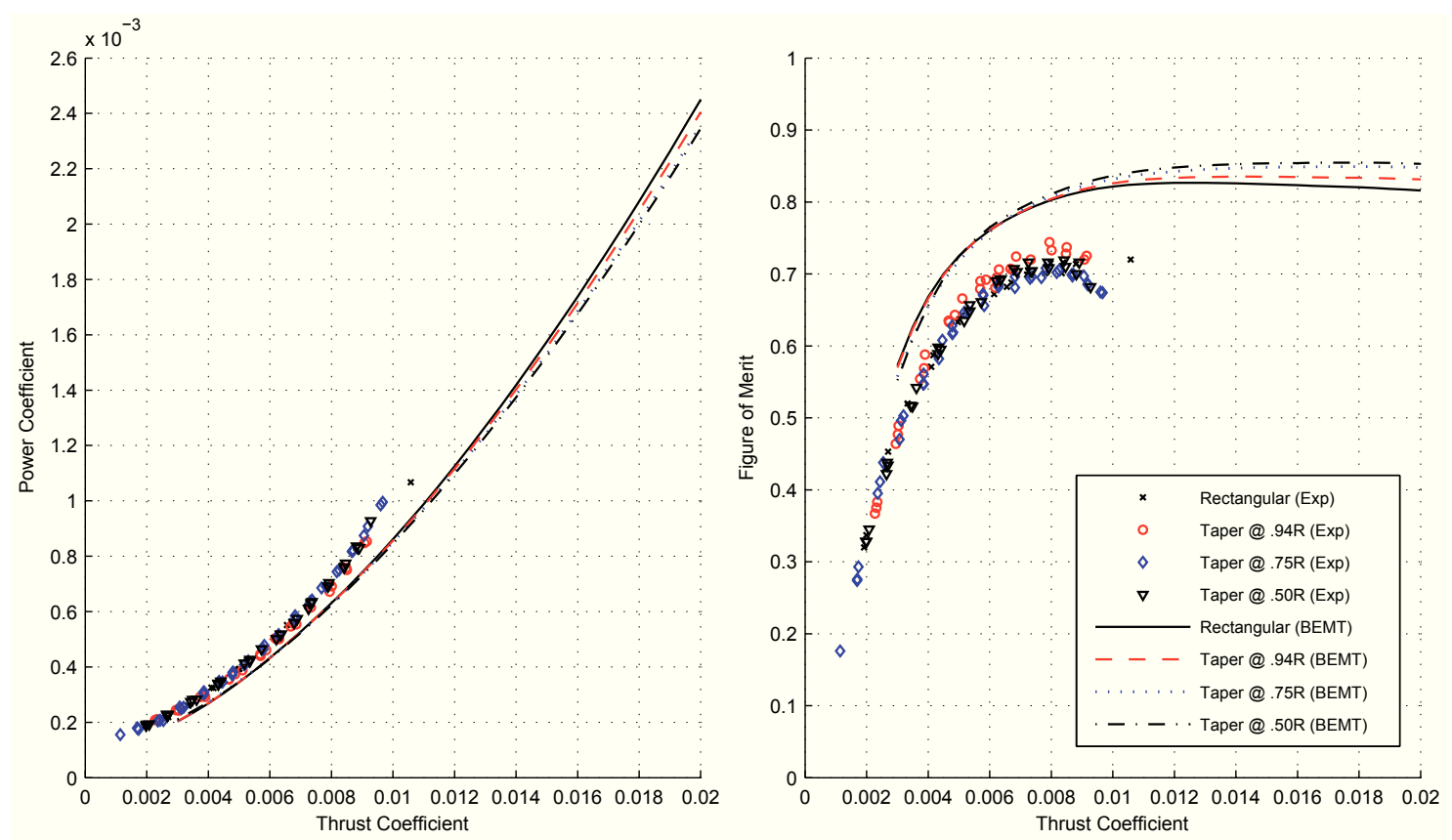

Figure B.3: Power coefficient and figure of merit simulated using the hover module compared to experimental results for rotors with different spanwise taper initiation [23]. 
maximum figure of merit is also shown to increase with decreasing taper initialization location from approximately 0.012 for the baseline rotor to 0.018 for the rotor featuring taper over $50 \%$ of its span.

Table B.3: Test condition and rotor characteristics: variation of spanwise taper initialization [23].

\begin{tabular}{ccccc}
\hline Parameter & Baseline & Taper(0.94R) & Taper(0.75R) & Taper(0.50R) \\
\hline \hline Number of Blades & 4 & 4 & 4 & 4 \\
Diameter (m) & 1.65 & 1.65 & 1.65 & 1.65 \\
Rotorspeed (rpm) & 2500 & 2500 & 2500 & 2500 \\
Tip Speed (m/s) & 216.1 & 216.1 & 216.1 & 216.1 \\
Solidity & 0.0977 & 0.0977 & 0.0977 & 0.0977 \\
Twist $\left({ }^{\circ}\right)$ & -13 & -13 & -13 & -13 \\
Taper & Rectangular & $3: 1 @ 0.94 R$ & $3: 1 @ 0.75 R$ & $3: 1 @ 0.50 R$ \\
& RC(4)-10, & RC(4)-10, & RC(4)-10, & RC(4)-10, \\
Airfoils & RC(3)-10 & RC(3)-10 & RC(3)-10 & RC(3)-10 \\
& $\&$ RC(3)-08 & $\&$ RC(3)-08 & $\& \mathrm{RC}(3)-08$ & $\& \mathrm{RC}(3)-08$ \\
\hline
\end{tabular}


Reference [65] presents experimental performance measurements made during full scale studies of two coaxial rotors with rotor diameters of $7.62 \mathrm{~m}(25 \mathrm{ft})$. Published data includes both single and coaxial rotor operation trials. Only data related to single rotor operation is considered in this comparison. The two rotors had different solidities and were not directly compared to one another. Both rotors featured symmetrical NACA four digit series airfoils of varying thickness along the rotor span. Rotor 1 had a nonlinear taper distribution while the planform of Rotor 2 was rectangular. The experimental data shown in Figure B.4 was reproduced from figures in Reference [65]. Individual data points do not coincide with the measured data points.

The experimental thrust coefficient range did not extend high enough to observe the peak in figure of merit. Similar to the scaled rotor tests, the hover module predicted lower power coefficients and higher figure of merit values than measured during the
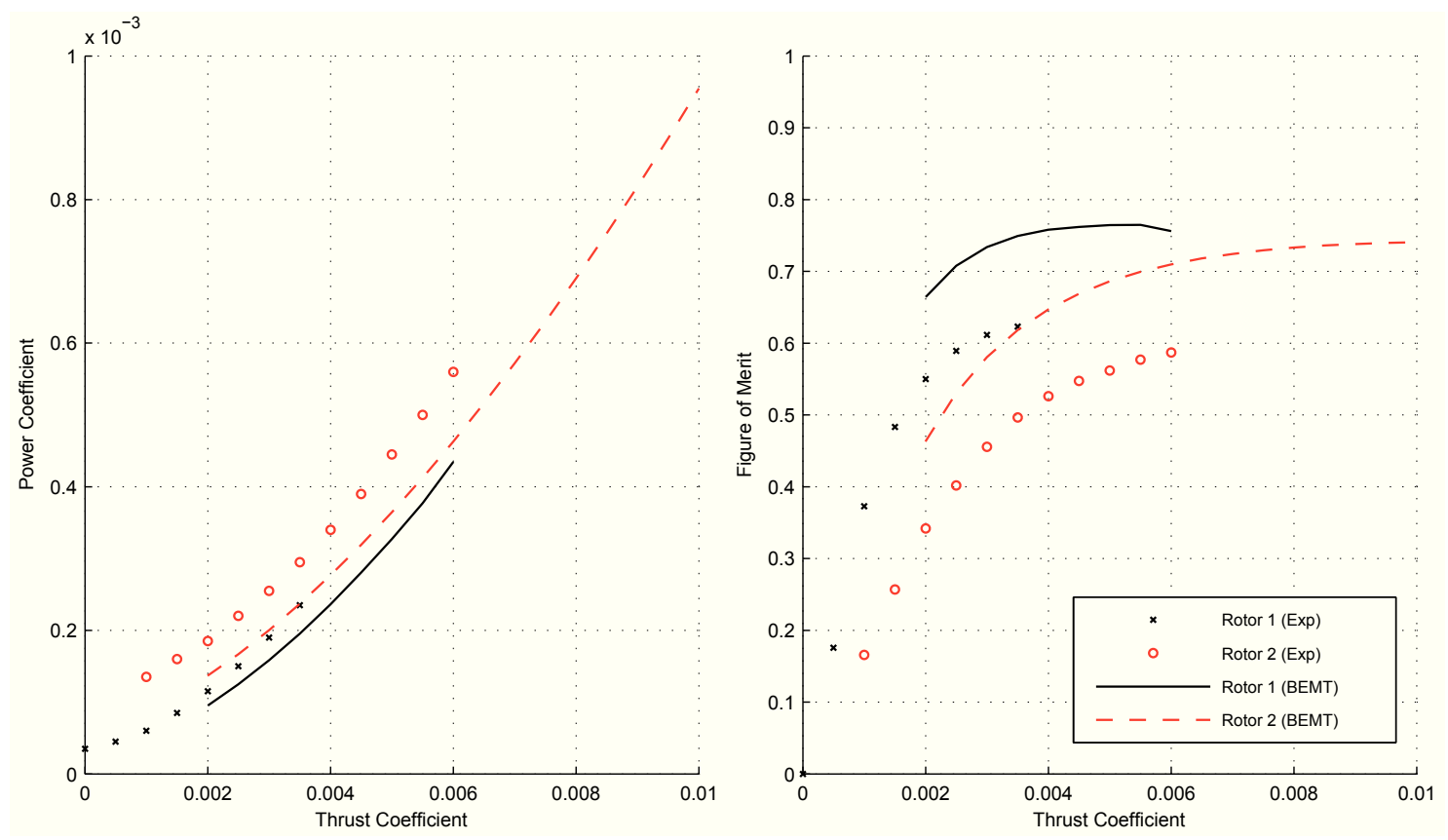

Figure B.4: Power coefficient and figure of merit simulated using the hover module compared to full scale experimental results [65]. 
experiment.

Table B.4: Test condition and rotor characteristics: full scale results [65].

\begin{tabular}{ccc}
\hline Parameter & Rotor 1 & Rotor 2 \\
\hline \hline Number of Blades & 2 & 2 \\
Diameter (m) & 7.62 & 7.62 \\
Rotorspeed (rpm) & 382.0 & 200.1 \\
Tip Speed ( $\mathrm{m} / \mathrm{s})$ & 152.4 & 79.9 \\
Solidity & 0.027 & 0.076 \\
Twist $\left({ }^{\circ}\right)$ & 0 & 0 \\
Taper & $3: 1$ @ 0.94R & Rectangular \\
Airfoils & NACA 4-digit & NACA 4-digit \\
& symmetric & symmetric \\
\hline
\end{tabular}




\section{Appendix C}

\section{Blade Dynamics - Equations of Motion}

While general blade flapping and lead-lagging equations of motion are published in helicopter related textbooks such as References [13] and [30], authors may apply different assumptions and use different reference systems. Therefore, the equations of motion for a few rotor configurations were derived from first principles. First, a fully articulated rotor was considered and the effect of relative placement of the flapping and lead-lag hinges was analysed as well as the addition of a flapping hinge spring and a lead-lag damper. Then, in order to more realistically simulate an $\mathrm{r} / \mathrm{c}$ helicopter rotor equations for teetering rotors were also derived. Finally, an alternative rigid $\mathrm{r} / \mathrm{c}$ rotor hub configuration is discussed.

As mentioned in Section 3.3, only rigid blade motion is considered in the Qoptr forward flight module. For the purpose of deriving the equations of motion the blade mass is assumed to be distributed one-dimensionally spanwise along a straight line passing through the outermost blade motion hinge. Using the Lagrange Equation approach the equations of motion for a mass element of the blade were derived and simplified. The results are integrated over the blade span to determine the equations of motion for the complete blade. The position of the blade mass is defined with respect to the rotating reference frame that was introduced in Section 3.3 for the direction of the aerodynamic 
forces $F_{x}, F_{r}$ and $F_{z}$. This reference frame rotates about the z-axis at rotorspeed $\Omega$. Unit vectors $\hat{i}, \hat{j}$ and $\hat{k}$ are used for the principle directions $x, r$ and $z$, respectively, where $\mathrm{x}$ is oriented tangentially opposite to the rotation of the rotor, $r$ points in the radial direction and $\mathrm{z}$ is parallel to the shaft axis. Rotorspeed $\Omega$ is assumed constant and the two independent degrees of freedom are the flap deflection $\beta$ and the lag deflection $\zeta$.

\section{Fully Articulated Rotor Hub}

The first derivation considers a fully articulated rotor blade, where the lead-lag hinge $e_{l l}$ is located inboard of the flapping hinge $e_{f l}$ as shown in Figure C.1. Hinge locations $e_{l l}$ and $e_{f l}$ represent non-dimensionalized values relative to rotor radius $\mathrm{R}$.

Position vector $\vec{P}_{\mathrm{d} m}$ points from the origin of the reference system to the mass element

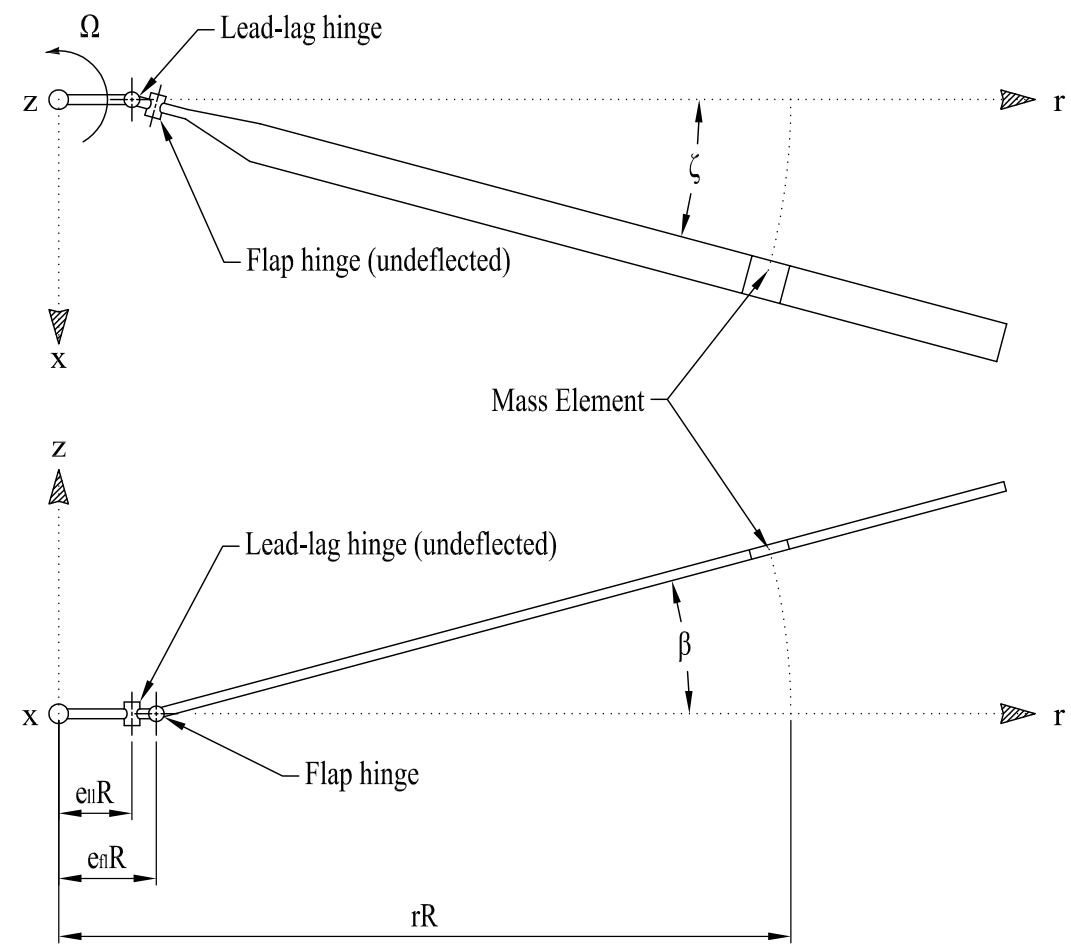

Figure C.1: Fully articulated rotor hub layout in hub-based rotating reference frame. 
$\mathrm{d} m$ located spanwise at $r R^{1}$.

$$
\begin{aligned}
\vec{P}_{\mathrm{d} m}=R & \left(\left(e_{f l}-e_{l l}\right) \sin (\zeta)+\left(r-e_{f l}\right) \sin (\zeta) \cos (\beta)\right) \hat{i} \\
& +R\left(e_{l l}+\left(e_{f l}-e_{l l}\right) \cos (\zeta)+\left(r-e_{f l}\right) \cos (\zeta) \cos (\beta)\right) \hat{j} \\
& +R\left(r-e_{f l}\right) \sin (\beta) \hat{k}
\end{aligned}
$$

The velocity of mass $\mathrm{d} m$ relative to the inertial hub-based reference frame can then be determined from the position vector $\vec{P}_{\mathrm{d} m}$ and the rotation of the rotating reference frame $\vec{\omega}$.

$$
\begin{aligned}
& \vec{V}_{\mathrm{d} m}=\frac{\mathrm{d}}{\mathrm{d} t}\left(\vec{P}_{\mathrm{d} m}\right)+\vec{\omega} \times \vec{P}_{\mathrm{d} m} \\
& \vec{V}_{\mathrm{d} m}=\left[R\left(e_{f l}-e_{l l}\right) \cos (\zeta) \dot{\zeta}+R\left(r-e_{f l}\right) \cos (\zeta) \cos (\beta) \dot{\zeta}\right. \\
& -R\left(r-e_{f l}\right) \sin (\zeta) \sin (\beta) \dot{\beta} \\
& \left.-R e_{l l} \Omega-R\left(e_{f l}-e_{l l}\right) \cos (\zeta) \Omega-R\left(r-e_{f l}\right) \cos (\zeta) \cos (\beta) \Omega\right] \hat{i} \\
& +\left[-R\left(e_{f l}-e_{l l}\right) \sin (\zeta) \dot{\zeta}-R\left(r-e_{f l}\right) \sin (\zeta) \cos (\beta) \dot{\zeta}\right. \\
& -R\left(r-e_{f l}\right) \cos (\zeta) \sin (\beta) \dot{\beta} \\
& \left.+R\left(e_{f l}-e_{l l}\right) \sin (\zeta) \Omega+R\left(r-e_{f l}\right) \sin (\zeta) \cos (\beta) \Omega\right] \hat{j} \\
& +R\left(r-e_{f l}\right) \cos (\beta) \dot{\beta} \hat{k}
\end{aligned}
$$

The general form of the Lagrange equation for degree of freedom $q_{i}$ is shown in Equation C.4 [69, p. 286].

$$
\frac{\mathrm{d}}{\mathrm{d} t}\left(\frac{\partial E_{k}}{\partial \dot{q}_{i}}\right)-\frac{\partial E_{k}}{\partial q_{i}}+\frac{\partial E_{p}}{\partial q_{i}}=Q_{q_{i}}
$$

Kinetic energy $E_{k}$ and potential energy $E_{p}$ of the mass element can be determined

\footnotetext{
${ }^{1}$ Equation C. 1 applies to positive values of $r$, where $r>e_{f l}$.
} 
from the velocity and position vector of the mass element. For the potential energy calculation it is assumed that the gravity vector is approximately aligned with the zaxis and that components of gravity orthogonal to this direction are negligible.

$$
\begin{gathered}
E_{k}=\frac{1}{2} \mathrm{~d} m\left(\vec{V}_{\mathrm{d} m} \cdot \vec{V}_{\mathrm{d} m}\right) \\
E_{p}=\vec{g} \cdot \vec{P}_{\mathrm{d} m} \\
E_{k}=\frac{1}{2} \mathrm{~d} m R^{2}\left[\left(\left(r-e_{f l}\right) \cos (\beta)+\left(e_{f l}-e_{l l}\right)\right)^{2} \dot{\zeta}^{2}+\left(r-e_{f l}\right)^{2} \dot{\beta}^{2}\right. \\
-2\left(\left(r-e_{f l}\right) \cos (\beta)+\left(e_{f l}-e_{l l}\right)\right)^{2} \dot{\zeta} \Omega \\
-2\left(\left(r-e_{f l}\right) \cos (\beta)+\left(e_{f l}-e_{l l}\right)\right) e_{l l} \cos (\zeta) \dot{\zeta} \Omega \\
+2\left(r-e_{f l}\right) e_{l l} \sin (\zeta) \sin (\beta) \dot{\beta} \Omega+\left(\left(r-e_{f l}\right) \cos (\beta)+\left(e_{f l}-e_{l l}\right)\right)^{2} \Omega^{2} \\
\left.+2\left(\left(r-e_{f l}\right) \cos (\beta)+\left(e_{f l}-e_{l l}\right)\right) e_{l l} \cos (\zeta) \Omega^{2}+e_{l l}^{2} \Omega^{2}\right] \\
E_{p}=\mathrm{d} m g R\left(r-e_{f l}\right) \sin (\beta)
\end{gathered}
$$

The non-conservative work terms account for the effect of the aerodynamic forces and were determined as shown in Equation C.9.

$$
\begin{gathered}
Q_{q_{i}}=\vec{F} \cdot \frac{\partial}{\partial q_{i}}\left(\vec{P}_{\mathrm{d} m}\right) \\
Q_{\beta}=-\mathrm{d} F_{x} R\left(r-e_{f l}\right) \sin (\zeta) \sin (\beta)-\mathrm{d} F_{r} R\left(r-e_{f l}\right) \cos (\zeta) \sin (\beta) \\
+\mathrm{d} F_{z} R\left(r-e_{f l}\right) \cos (\beta)
\end{gathered}
$$




$$
\begin{aligned}
Q_{\zeta}=\mathrm{d} F_{x} R & \left(\left(r-e_{f l}\right) \cos (\beta)+\left(e_{f l}-e_{l l}\right)\right) \cos (\zeta) \\
& -\mathrm{d} F_{r} R\left(\left(r-e_{f l}\right) \cos (\beta)+\left(e_{f l}-e_{l l}\right)\right) \sin (\zeta)
\end{aligned}
$$

Starting with the flapping motion the individual terms on the left hand side of Equation C.4 evaluate to:

$$
\begin{aligned}
& \frac{\mathrm{d}}{\mathrm{d} t}\left(\frac{\partial E_{k}}{\partial \dot{\beta}}\right)=\mathrm{d} m R^{2}\left[\left(r-e_{f l}\right) e_{l l} \cos (\zeta) \sin (\beta) \dot{\zeta} \Omega\right. \\
&\left.+\left(r-e_{f l}\right) e_{l l} \sin (\zeta) \cos (\beta) \dot{\beta} \Omega+\left(r-e_{f l}\right)^{2} \ddot{\beta}\right] \\
& \frac{\partial E_{k}}{\partial \beta}=\mathrm{d} m R^{2}\left[\left(\left(r-e_{f l}\right) \cos (\beta)+\left(e_{f l}-e_{l l}\right)\right)\left(r-e_{f l}\right) \sin (\beta) \dot{\zeta}(2 \Omega-\dot{\zeta})\right. \\
& \quad+\left(r-e_{f l}\right) e_{l l} \cos (\zeta) \sin (\beta) \dot{\zeta} \Omega+\left(r-e_{f l}\right) e_{l l} \sin (\zeta) \cos (\beta) \dot{\beta} \Omega \\
&-\left(\left(r-e_{f l}\right) \cos (\beta)+\left(e_{f l}-e_{l l}\right)\right)\left(r-e_{f l}\right) \sin (\beta) \Omega^{2} \\
&\left.-e_{l l}\left(r-e_{f l}\right) \sin (\beta) \cos (\zeta) \Omega^{2}\right] \\
& \frac{\partial E_{p}}{\partial \beta}=\mathrm{d} m g R\left(r-e_{f l}\right) \cos (\beta)
\end{aligned}
$$

Combining these terms with the non-conservative work term for flapping $Q_{\beta}$ gives the Lagrange Equation for the flapping degree of freedom:

$$
\begin{aligned}
R^{2} \mathrm{~d} m & {\left[\left(r-e_{f l}\right)^{2} \ddot{\beta}-\left(\left(r-e_{f l}\right) \cos (\beta)+\left(e_{f l}-e_{l l}\right)\right)\left(r-e_{f l}\right) \sin (\beta) \dot{\zeta}(2 \Omega-\dot{\zeta})\right.} \\
+ & \left.\left(r-e_{f l}\right) e_{l l} \sin (\beta) \cos (\zeta) \Omega^{2}+\left(\left(r-e_{f l}\right) \cos (\beta)+\left(e_{f l}-e_{l l}\right)\right)\left(r-e_{f l}\right) \sin (\beta) \Omega^{2}\right] \\
+ & R \mathrm{~d} m g\left(r-e_{f l}\right) \cos (\beta) \\
= & -\mathrm{d} F_{x} R\left(r-e_{f l}\right) \sin (\zeta) \sin (\beta)-\mathrm{d} F_{r} R\left(r-e_{f l}\right) \cos (\zeta) \sin (\beta) \\
\quad & +\mathrm{d} F_{z} R\left(r-e_{f l}\right) \cos (\beta)
\end{aligned}
$$


Repeating the procedure for the lead-lag motion gives:

$$
\begin{aligned}
& \frac{\mathrm{d}}{\mathrm{d} t}\left(\frac{\partial E_{k}}{\partial \dot{\zeta}}\right)=\mathrm{d} m R^{2}\left[\left(\left(r-e_{f l}\right) \cos (\beta)+\left(e_{f l}-e_{l l}\right)\right)^{2} \ddot{\zeta}\right. \\
&+2\left(\left(r-e_{f l}\right) \cos (\beta)+\left(e_{f l}-e_{l l}\right)\right)\left(r-e_{f l}\right) \sin (\beta) \dot{\beta}(\Omega-\dot{\zeta}) \\
&\left.+\left(r-e_{f l}\right) e_{l l} \cos (\zeta) \sin (\beta) \dot{\beta} \Omega+\left(\left(r-e_{f l}\right) \cos (\beta)+\left(e_{f l}-e_{l l}\right)\right) e_{l l} \sin (\zeta) \dot{\zeta} \Omega\right] \\
& \frac{\partial E_{k}}{\partial \zeta}=\mathrm{d} m R^{2}\left[+\left(r-e_{f l}\right) e_{l l} \cos (\zeta) \sin (\beta) \dot{\beta} \Omega\right. \\
&\left.-\left(\left(r-e_{f l}\right) \cos (\beta)+\left(e_{f l}-e_{l l}\right)\right) e_{l l} \sin (\zeta) \Omega(\Omega-\dot{\zeta})\right] \\
& \quad \frac{\partial E_{p}}{\partial \zeta}=0
\end{aligned}
$$

Combining the lead-lag terms as shown in Equation C.4 produces the Lagrange Equation for the lead-lag degree of freedom.

$$
\begin{aligned}
& R^{2} \mathrm{~d} m\left[\left(\left(r-e_{f l}\right) \cos (\beta)+\left(e_{f l}-e_{l l}\right)\right)^{2} \ddot{\zeta}\right. \\
& +2\left(\left(r-e_{f l}\right) \cos (\beta)+\left(e_{f l}-e_{l l}\right)\right)\left(r-e_{f l}\right) \sin (\beta) \dot{\beta}(\Omega-\dot{\zeta}) \\
& \left.+\left(\left(r-e_{f l}\right) \cos (\beta)+\left(e_{f l}-e_{l l}\right)\right) e_{l l} \sin (\zeta) \Omega^{2}\right] \\
& =\mathrm{d} F_{x} R\left(\left(r-e_{f l}\right) \cos (\beta)+\left(e_{f l}-e_{l l}\right)\right) \cos (\zeta) \\
& \quad-\mathrm{d} F_{r} R\left(\left(r-e_{f l}\right) \cos (\beta)+\left(e_{f l}-e_{l l}\right)\right) \sin (\zeta)
\end{aligned}
$$

Due to the complexity of the Lagrange Equations several simplifying assumptions were employed. Rotor flap and lag deflection amplitudes are usually small, justifying small angle approximations. Only first order terms were kept, i.e.:

$$
\begin{array}{ll}
\sin \beta=\beta & \cos \beta=1 \\
\sin \zeta=\zeta & \cos \zeta=1
\end{array}
$$


Equations C.21 and C.22 show the two Lagrange Equations after the small angle approximation.

$$
\begin{aligned}
R^{2} \mathrm{~d} m & {\left[\left(r-e_{f l}\right)^{2} \ddot{\beta}-\left(r-e_{f l}\right)\left(r-e_{l l}\right) \beta \dot{\zeta}(2 \Omega-\dot{\zeta})+r\left(r-e_{f l}\right) \Omega^{2} \beta\right] } \\
& =-\mathrm{d} F_{x} R\left(r-e_{f l}\right) \zeta \beta-\mathrm{d} F_{r} R\left(r-e_{f l}\right) \beta+\left(\mathrm{d} F_{z}-\mathrm{d} m g\right) R\left(r-e_{f l}\right) \\
R^{2} \mathrm{~d} m & {\left[\left(r-e_{l l}\right)^{2} \ddot{\zeta}+2\left(r-e_{f l}\right)\left(r-e_{l l}\right) \beta \dot{\beta}(\Omega-\dot{\zeta})+\left(r-e_{l l}\right) e_{l l} \Omega^{2} \zeta\right] } \\
& =\mathrm{d} F_{x} R\left(r-e_{l l}\right)-\mathrm{d} F_{r} R\left(r-e_{l l}\right) \zeta
\end{aligned}
$$

Using a relative magnitude comparison further terms were eliminated according to the following criteria, resulting in the final equations for the mass element $\mathrm{d} m$ with respect to the flapping and lead-lagging degrees of freedom $\beta$ and $\zeta$ (Eqs. C.26 and C.27).

$$
\begin{gathered}
|\dot{\zeta}| \ll|\Omega| \\
|\mathrm{d} m g| \ll\left|\mathrm{d} F_{z}\right| \\
\zeta \beta \approx 0 \\
R^{2} \mathrm{~d} m\left[\left(r-e_{f l}\right)^{2} \ddot{\beta}-2\left(r-e_{f l}\right)\left(r-e_{l l}\right) \Omega \beta \dot{\zeta}+r\left(r-e_{f l}\right) \Omega^{2} \beta\right] \\
=-\mathrm{d} F_{r} R\left(r-e_{f l}\right) \beta+\mathrm{d} F_{z} R\left(r-e_{f l}\right) \\
R^{2} \mathrm{~d} m\left[\left(r-e_{l l}\right)^{2} \ddot{\zeta}+2\left(r-e_{f l}\right)\left(r-e_{l l}\right) \Omega \beta \dot{\beta}+\left(r-e_{l l}\right) e_{l l} \Omega^{2} \zeta\right] \\
=\mathrm{d} F_{x} R\left(r-e_{l l}\right)-\mathrm{d} F_{r} R\left(r-e_{l l}\right) \zeta
\end{gathered}
$$

The expressions derived for an element of mass are then integrated along the span of 
the blade. For the integration $\mathrm{d} m$ is replaced with $m_{d} \mathrm{~d} r$, where $m_{d}$ is the spanwise linear density in units of mass per unit. Similarly $\mathrm{d} F_{x}, \mathrm{~d} F_{r}$ and $\mathrm{d} F_{z}$ are replaced with $F_{x} \mathrm{~d} r$, $F_{r} \mathrm{~d} r$ and $F_{z} \mathrm{~d} r$, respectively. For a fully articulated hub design the flapping motion is integrated from $e_{f l} R$ to $R$, while the lead-lag motion is integrated from $e_{l l} R$ to $R^{2}$.

$$
\begin{aligned}
& R^{2} \int_{e_{f l}}^{1} m_{d}\left(r-e_{f l}\right)^{2} \mathrm{~d} r \ddot{\beta}-2 R^{2} \int_{e_{f l}}^{1} m_{d}\left(r-e_{f l}\right)\left(r-e_{l l}\right) \mathrm{d} r \Omega \beta \dot{\zeta}+R^{2} \int_{e_{f l}}^{1} m_{d} r\left(r-e_{f l}\right) \mathrm{d} r \Omega^{2} \beta \\
& \quad=R \int_{e_{f l}}^{1}\left(r-e_{f l}\right)\left(F_{z}-F_{r} \beta\right) \mathrm{d} r \\
& R^{2} \int_{e_{l l}}^{1} m_{d}\left(r-e_{l l}\right)^{2} \mathrm{~d} r \ddot{\zeta}+2 R^{2} \int_{e_{f l}}^{1} m_{d}\left(r-e_{f l}\right)\left(r-e_{l l}\right) \mathrm{d} r \Omega \beta \dot{\beta}+R^{2} e_{l l} \int_{e_{l l}}^{1} m_{d}\left(r-e_{l l}\right) \mathrm{d} r \Omega^{2} \zeta \\
& \quad=R \int_{e_{l l}}^{1}\left(r-e_{l l}\right)\left(F_{x}-F_{r} \zeta\right) \mathrm{d} r
\end{aligned}
$$

The blade moments of inertia about the flapping and lead-lagging hinges have the following definitions (neglecting coupled flap-lag effects).

$$
\begin{gathered}
I_{\beta}=R^{2} \int_{e_{f l}}^{1} m_{d}\left(r-e_{f l}\right)^{2} \mathrm{~d} r \\
I_{\zeta}=R^{2} \int_{e_{l l}}^{1} m_{d}\left(r-e_{l l}\right)^{2} \mathrm{~d} r
\end{gathered}
$$

Further defining $I_{\text {Cor }}$ to account for the integrals of the Coriolis terms in Equations C.28 and C.29 and non-dimensional natural flapping and lead-lagging frequencies $v_{\beta}$ and $v_{\zeta}$ as

$$
I_{C o r}=R^{2} \int_{e_{f l}}^{1} m_{d}\left(r-e_{f l}\right)\left(r-e_{l l}\right) \mathrm{d} r
$$

\footnotetext{
${ }^{2}$ Coriolis terms are integrated from the most outward hinge outward, in this case from $e_{f l} R$ to $R$.
} 
APPENDIX C. BLADE DYNAMICS - EQUATIONS OF MOTION

$$
\begin{gathered}
v_{\beta}^{2}=1+\frac{R^{2} e_{f l} \int_{e_{f l}}^{1} m_{d}\left(r-e_{f l}\right) \mathrm{d} r}{I_{\beta}} \\
v_{\zeta}^{2}=\frac{R^{2} e_{l l} \int_{e_{l l}}^{1} m_{d}\left(r-e_{l l}\right) \mathrm{d} r}{I_{\zeta}}
\end{gathered}
$$

allows the equations of motion to be rewritten as shown in Equations C.35 and C.36.

$$
\begin{aligned}
& I_{\beta}\left(\ddot{\beta}+v_{\beta}^{2} \Omega^{2} \beta-2 \frac{I_{C o r}}{I_{\beta}} \Omega \beta \dot{\zeta}\right)=R \int_{e_{f l}}^{1}\left(r-e_{f l}\right)\left(F_{z}-F_{r} \beta\right) \mathrm{d} r \\
& I_{\zeta}\left(\ddot{\zeta}+v_{\zeta}^{2} \Omega^{2} \zeta+2 \frac{I_{C o r}}{I_{\zeta}} \Omega \beta \dot{\beta}\right)=R \int_{e_{l l}}^{1}\left(r-e_{l l}\right)\left(F_{x}-F_{r} \zeta\right) \mathrm{d} r
\end{aligned}
$$

As a final step the velocity and acceleration terms are non-dimensionalized with respect to rotorspeed $\Omega$ (see Eqs. 3.24 and 3.25) and the equations are solved for the flapping and lead-lag acceleration, respectively.

$$
\begin{aligned}
& \beta^{* *}=-v_{\beta}^{2} \beta+2 \frac{I_{C o r}}{I_{\beta}} \beta \zeta^{*}+\frac{R}{I_{\beta} \Omega^{2}} \int_{e_{f l}}^{1}\left(r-e_{f l}\right)\left(F_{z}-F_{r} \beta\right) \mathrm{d} r \\
& \zeta^{* *}=-v_{\zeta}^{2} \zeta-2 \frac{I_{C o r}}{I_{\zeta}} \beta \beta^{*}+\frac{R}{I_{\zeta} \Omega^{2}} \int_{e_{l l}}^{1}\left(r-e_{l l}\right)\left(F_{x}-F_{r} \zeta\right) \mathrm{d} r
\end{aligned}
$$

Considering the final equations of motion for a fully articulated rotor blade the terms can be classified from left to right as inertial, centrifugal, Coriolis and aerodynamic force effects.

The above procedure was repeated for a fully articulated rotor featuring the flapping hinge radially inward with respect to the lead-lag hinge, i.e. $e_{f l}<e_{l l}$. The resulting Lagrange Equations simplified for small angles are shown in Equations C.39 and C.40.

$$
\begin{aligned}
R^{2} \mathrm{~d} m & {\left[\left(r-e_{f l}\right)^{2} \ddot{\beta}-2\left(r-e_{f l}\right)\left(r-e_{l l}\right) \dot{\zeta}(\Omega \beta+\zeta \dot{\beta})+r\left(r-e_{f l}\right) \Omega^{2} \beta\right] } \\
& =-\mathrm{d} F_{r} R\left(r-e_{f l}\right) \beta+\left(\mathrm{d} F_{z}-\mathrm{d} m g\right) R\left(r-e_{f l}\right)
\end{aligned}
$$




$$
\begin{aligned}
R^{2} \mathrm{~d} m & {\left[\left(r-e_{l l}\right)^{2} \ddot{\zeta}+\left(r-e_{l l}\right)\left(r-e_{f l}\right) \dot{\beta}(2 \Omega \beta+\zeta \dot{\beta})+\left(r-e_{l l}\right) e_{l l} \Omega^{2} \zeta\right] } \\
& =\mathrm{d} F_{x} R\left(r-e_{l l}\right)-\mathrm{d} F_{r} R\left(r-e_{l l}\right) \zeta-\left(\mathrm{d} F_{z}-\mathrm{d} m g\right) R\left(r-e_{l l}\right) \beta \zeta
\end{aligned}
$$

Adding the following assumptions to the magnitude comparison based assumptions stated above (Equations C.23 to C.25)

$$
|\zeta \dot{\beta}| \ll|\Omega \beta|
$$

results in the same equations of motion obtained for the original fully articulated hub with $e_{l l}<e_{f l}$. Hence, any effects due to relative spanwise hinge placement are expected to be small and neglected in the equations of motion implemented in the forward flight module.

\section{Addition of a Flapping Hinge Spring and a Lead-lag Hinge Damper}

Fully articulated rotors may employ additional elements affecting the dynamics of the rigid blade motion. Two components considered here are the addition of a simple flapping spring and a simple lead-lag damper. Since the derivation is very similar to the process outlined above only significant differences will be discussed.

To determine the effect of an added spring acting about the flapping hinge with stiffness $K_{\beta}$ - in units of torque per radian - and neutral position at precone angle $\beta_{p}$ Equation C.28 is modified to

$$
\begin{aligned}
& R^{2} \int_{e_{f l}}^{1} m_{d}\left(r-e_{f l}\right)^{2} \mathrm{~d} r \ddot{\beta}-2 R^{2} \int_{e_{f l}}^{1} m_{d}\left(r-e_{f l}\right)\left(r-e_{l l}\right) \mathrm{d} r \Omega \beta \dot{\zeta}+R^{2} \int_{e_{f l}}^{1} m_{d} r\left(r-e_{f l}\right) \mathrm{d} r \Omega^{2} \beta \\
& \quad+K_{\beta}\left(\beta-\beta_{p}\right)=R \int_{e_{f l}}^{1}\left(r-e_{f l}\right)\left(F_{z}-F_{r} \beta\right) \mathrm{d} r
\end{aligned}
$$


which alters the non-dimensional natural flapping frequency $v_{\beta}$ as shown in Equation C.43.

$$
v_{\beta}^{2}=1+\frac{R^{2} e_{f l} \int_{e_{f l}}^{1} m_{d}\left(r-e_{f l}\right) \mathrm{d} r}{I_{\beta}}+\frac{K_{\beta}}{I_{\beta} \Omega^{2}}
$$

The final flapping equation of motion for a fully articulated rotor with a flapping hinge spring is given in Equation C.44.

$$
\beta^{* *}=-v_{\beta}^{2} \beta+2 \frac{I_{C o r}}{I_{\beta}} \beta \zeta^{*}+\frac{K_{\beta}}{I_{\beta} \Omega^{2}} \beta_{p}+\frac{R}{I_{\beta} \Omega^{2}} \int_{e_{f l}}^{1}\left(r-e_{f l}\right)\left(F_{z}-F_{r} \beta\right) \mathrm{d} r
$$

Note that Equations C.43 and C.44 both reduce to the previously derived forms (Eqs. C.33 and C.37) when $K_{\beta}$ is set to zero. The lead-lag equation of motion is not affected by the addition of a flapping hinge spring.

In a similar fashion a lead-lag damper can be added to the lag equation. A dynamic damper providing a moment proportional to the rotational velocity $\dot{\zeta}$ with damping constant $C_{\zeta}$ - in units of torque per radian per second - was considered. Equation C.29 becomes

$$
\begin{aligned}
& R^{2} \int_{e_{l l}}^{1} m_{d}\left(r-e_{l l}\right)^{2} \mathrm{~d} r \ddot{\zeta}+2 R^{2} \int_{e_{l l}}^{1} m_{d}\left(r-e_{f l}\right)\left(r-e_{l l}\right) \mathrm{d} r \Omega \beta \dot{\beta}+R^{2} e_{l l} \int_{e_{l l}}^{1} m_{d}\left(r-e_{l l}\right) \mathrm{d} r \Omega^{2} \zeta \\
& \quad=-C_{\zeta} \dot{\zeta}+R \int_{e_{l l}}^{1}\left(r-e_{l l}\right)\left(F_{x}-F_{r} \zeta\right) \mathrm{d} r .
\end{aligned}
$$

Rearranging the equation as above to isolate $\zeta^{* *}$ the final lag degree of freedom equation of motion for a blade on a fully articulated rotor hub with a lead-lag damper is produced as shown in Equation C.46.

$$
\zeta^{* *}=-v_{\zeta}^{2} \zeta-2 \frac{I_{C o r}}{I_{\zeta}} \beta \beta^{*}-\frac{C_{\zeta}}{I_{\zeta} \Omega} \zeta^{*}+\frac{R}{I_{\zeta} \Omega^{2}} \int_{e_{l l}}^{1}\left(r-e_{l l}\right)\left(F_{x}-F_{r} \zeta\right) \mathrm{d} r
$$

Again, Equation C.46 can be shown to reduce to Equation C.38 when $C_{\zeta}$ is set to zero 
and the flapping equation of motion is not affected by the introduction of the lead-lag damper.

\section{Teetering Rotor Hub}

As most $\mathrm{r} / \mathrm{c}$ helicopters have two-bladed rotors and teetering rotor hubs the equation of motion for this arrangement were also derived. The teetering rotor shown in Figure C.2 with precone angle $\beta_{p}$ and non-dimensional undersling distance $e_{u s}$ was considered. The mass of the rotor is assumed to be distributed along the "v"-shape formed by the two blades extended to the rotor shaft, i.e. specific mass or inertia characteristics the rotor hub are not considered.

Teetering hubs on full-scale helicopters in general do not feature lead-lag hinges.

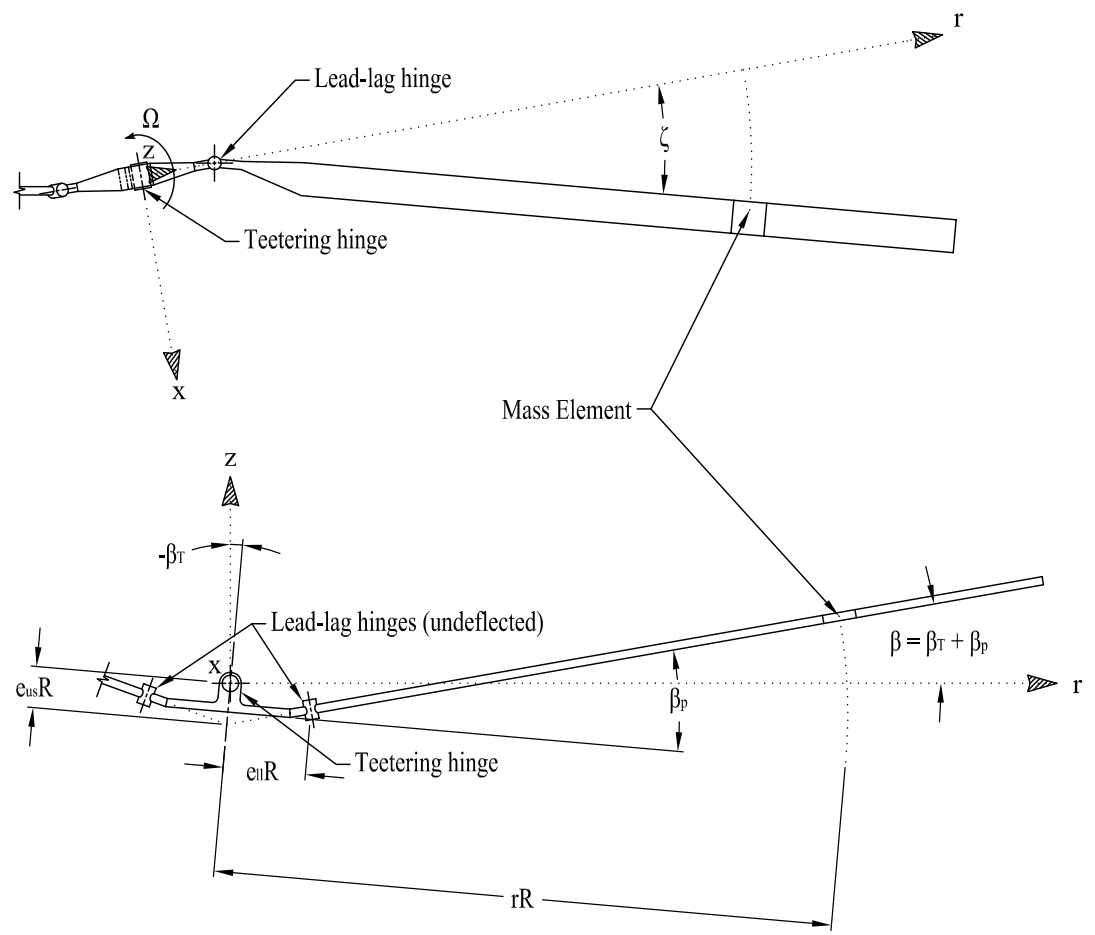

Figure C.2: Teetering rotor hub layout in hub-based rotating reference frame. 
Effects due to Coriolis accelerations are reduced using an underslung hub design and the remainder is transmitted to the rotor hub. $\mathrm{R} / \mathrm{c}$ helicopter blades on the other hand are typically attached to the rotor hub using a single bolt, which forms an effective lead-lag hinge. The lag deflection of one blade is independent of that of the other. The equations of motion derived here account for a lead-lag hinge, but may be reduced to a single teetering equation of motion, where the rotor blades are not allowed to lag and the rotor is "stiff in-plane".

For consistency in the simulation algorithm $\beta$ is still defined as the flapping inclination of the blade with respect to the hub plane. Since only rigid blade motion is considered, this angle is linearly related to the teetering hinge deflection $\beta_{T}$ as shown in Equation C.47. It follows that the flapping and teetering velocity and acceleration terms are equivalent.

$$
\begin{gathered}
\beta=\beta_{T}+\beta_{p} \\
\dot{\beta}=\dot{\beta}_{T} \\
\ddot{\beta}=\ddot{\beta}_{T}
\end{gathered}
$$

The position vector from the teetering hinge to a mass element of the blade at radial position $r R$ is defined in Equation C.503.

$$
\begin{aligned}
\vec{P}_{\mathrm{d} m}=R & \left(\left(r-e_{l l}\right) \sin (\zeta)\right) \hat{i} \\
& +R\left(e_{u s} \sin \left(\beta_{T}\right)+e_{l l} \cos \left(\beta_{T}+\beta_{p}\right)+\left(r-e_{l l}\right) \cos \left(\beta_{T}+\beta_{p}\right) \cos (\zeta)\right) \hat{j} \\
& +R\left(-e_{u s} \cos \left(\beta_{T}\right)+e_{l l} \sin \left(\beta_{T}+\beta_{p}\right)+\left(r-e_{l l}\right) \sin \left(\beta_{T}+\beta_{p}\right) \cos (\zeta)\right) \hat{k}
\end{aligned}
$$

The same procedure - as outlined above for the fully articulated rotor - was used to

\footnotetext{
${ }^{3}$ Equation C.50 applies to positive values of $r$, where $r>e_{l l}$.
} 
derive the Lagrange Equations. The treatment continues with the equations of motion for the teetering and lead-lag degrees of freedom after small angle approximations were applied as shown in Equations C.51 and C.52, respectively.

$$
\begin{aligned}
& R^{2} \mathrm{~d} m\left[\left(r^{2}+e_{u s}^{2}-2 \beta_{p} e_{u s} r\right) \ddot{\beta}_{T}-2\left(r-e_{l l}\right)\left(\left(r-\beta_{p} e_{u s}\right) \zeta \dot{\beta}_{T}+r \beta_{T} \Omega\right) \dot{\zeta}\right. \\
& +\left(r-e_{l l}\right) e_{u s}(2 \Omega-\dot{\zeta}) \dot{\zeta}-2\left(r-e_{l l}\right) r \beta_{p} \Omega \dot{\zeta} \\
& \left.+\left(r^{2}-e_{u s}^{2}+2 \beta_{p} e_{u s} r\right) \beta_{T} \Omega^{2}+\left(\beta_{p} r-e_{u s}\right) r \Omega^{2}\right] \\
& \quad=-\mathrm{d} F_{r} R\left(\left(\beta_{T}+\beta_{p}\right) r-e_{u s}\right)+\left(\mathrm{d} F_{z}-g \mathrm{~d} m\right) R\left(r-\left(\beta_{p} r-e_{u s}\right) \beta_{T}\right) \\
& R^{2} \mathrm{~d} m\left[\left(r-e_{l l}\right)^{2} \ddot{\zeta}+\left(r-e_{l l}\right)\left(\left(r-\beta_{p} e_{u s}\right) \zeta \dot{\beta}+2 r \beta_{T} \Omega\right) \dot{\beta}_{T}+2\left(r-e_{l l}\right)\left(\beta_{p} r-e_{u s}\right) \Omega \dot{\beta}_{T}\right. \\
& \left.+\left(r-e_{l l}\right) e_{l l} \Omega^{2} \zeta-\left(r-e_{l l}\right)\left(2 \beta_{p} r-e_{u s}\right) \beta_{T} \Omega^{2} \zeta\right] \\
& \quad=\mathrm{d} F_{x} R\left(r-e_{l l}\right)-\mathrm{d} F_{r} R\left(r-e_{l l}\right)\left(1-\beta_{p} \beta_{T}\right) \zeta-\left(\mathrm{d} F_{z}-g \mathrm{~d} m\right) R\left(r-e_{l l}\right)\left(\beta_{T}+\beta_{p}\right) \zeta
\end{aligned}
$$

Using a similar order of magnitude analysis to determine the relative impact of components of the main dynamic effects - inertial, centrifugal, Coriolis and aerodynamic the approximations stated below were applied to arrive at Equations C.58 and C.59.

$$
\begin{gathered}
|g \mathrm{~d} m| \ll\left|\mathrm{d} F_{z}\right| \\
\left|\left(r-\beta_{p} e_{u s}\right) \zeta \dot{\beta}\right| \ll\left|r \beta_{T} \Omega\right| \\
|\dot{\zeta}| \ll|\Omega| \\
\left|\beta_{p} \beta_{T}\right| \ll 1 \\
\beta \zeta \approx 0
\end{gathered}
$$


APPENDIX C. BLADE DYNAMICS - EQUATIONS OF MOTION

$$
\begin{aligned}
& R^{2} \mathrm{~d} m\left[\left(r^{2}+e_{u s}^{2}-2 \beta_{p} e_{u s} r\right) \ddot{\beta}_{T}-2 r\left(r-e_{l l}\right) \beta_{T} \Omega \dot{\zeta}-2\left(r-e_{l l}\right)\left(\beta_{p} r-e_{u s}\right) \Omega \dot{\zeta}\right. \\
& \left.+\left(r^{2}-e_{u s}^{2}+2 \beta_{p} e_{u s} r\right) \beta_{T} \Omega^{2}+\left(\beta_{p} r-e_{u s}\right) r \Omega^{2}\right] \\
& =-\mathrm{d} F_{r} R\left(\left(\beta_{T}+\beta_{p}\right) r-e_{u s}\right)+\mathrm{d} F_{z} R\left(r-\left(\beta_{p} r-e_{u s}\right) \beta_{T}\right) \\
& R^{2} \mathrm{~d} m\left[\left(r-e_{l l}\right)^{2} \ddot{\zeta}+2 r\left(r-e_{l l}\right) \beta_{T} \Omega \dot{\beta}_{T}+2\left(r-e_{l l}\right)\left(\beta_{p} r-e_{u s}\right) \Omega \dot{\beta}_{T}\right. \\
& \left.+\left(r-e_{l l}\right) e_{l l} \Omega^{2} \zeta-\left(r-e_{l l}\right)\left(2 \beta_{p} r-e_{u s}\right) \beta_{T} \Omega^{2} \zeta\right] \\
& \quad=\mathrm{d} F_{x} R\left(r-e_{l l}\right)-\mathrm{d} F_{r} R\left(r-e_{l l}\right) \zeta
\end{aligned}
$$

When Equations C.58 and C.59 of the teetering rotor mass element are compared to those previously derived for the fully articulated rotor (Eqs. C.26 and C.27), unmistakable similarities are observed. It can be shown that the teetering rotor mass element equations with $e_{u s}=0$ are largely equivalent to the fully articulated rotor mass element equations with a flapping hinge located on the shaft axis. Further considering the Coriolis and centrifugal acceleration parts of the equations shows that compared to the fully articulated rotor additional components purely in terms of geometric characteristics of the teetering rotor hub have appeared.

As before, the equations for a single mass element are integrated along the rotor span. The lagging motions of the two blades are independent of each other and the integration is therefore performed from the lead-lag hinge outward. As the flapping motion of the blades is coupled by the teetering hub the teetering equation of motion requires an integration from the tip of one blade to the tip of the other. The position vector for the teetering rotor mass element was defined for positive values of $r>e_{l l}$ only. For mass elements positioned radially inward with respect to the lead-lag hinge all $\zeta$-related terms are zero. The equations of motion for the opposing blade will also 
be slightly different from Equations C.58 and C.59, but it can be shown that the two sets of equations are equal if the following two substitutions are made.

$$
\begin{aligned}
& e_{l l}=-e_{l l} \\
& \beta_{p}=-\beta_{p}
\end{aligned}
$$

Subscripts $\psi$ and $\psi+\pi$ are used to indicate the azimuth location for terms related to the two opposing blades.

$$
\begin{aligned}
& R^{2} \int_{0}^{1} m_{d}\left(r^{2}+e_{u s}^{2}-2 \beta_{p} e_{u s} r\right) \mathrm{d} r \ddot{\beta}_{T}+R^{2} \int_{-1}^{0} m_{d}\left(r^{2}+e_{u s}^{2}+2 \beta_{p} e_{u s} r\right) \mathrm{d} r \ddot{\beta}_{T} \\
& -2 R^{2} \int_{e_{l l}}^{1} m_{d} r\left(r-e_{l l}\right) \mathrm{d} r \beta_{T} \Omega \dot{\zeta}_{\psi}-2 R^{2} \int_{e_{l l}}^{1} m_{d}\left(r-e_{l l}\right)\left(\beta_{p} r-e_{u s}\right) \mathrm{d} r \Omega \dot{\zeta}_{\psi} \\
& -2 R^{2} \int_{-1}^{-e_{l l}} m_{d} r\left(r+e_{l l}\right) \mathrm{d} r \beta_{T} \Omega \dot{\zeta}_{\psi+\pi}-2 R^{2} \int_{-1}^{-e_{l l}} m_{d}\left(r+e_{l l}\right)(-1)\left(\beta_{p} r+e_{u s}\right) \mathrm{d} r \Omega \dot{\zeta}_{\psi+\pi} \\
& +R^{2} \int_{0}^{1}\left(r^{2}-e_{u s}^{2}+2 \beta_{p} e_{u s} r\right) \mathrm{d} r \Omega^{2} \beta_{T}+R^{2} \int_{0}^{1} r\left(\beta_{p} r-e_{u s}\right) \mathrm{d} r \Omega^{2} \\
& +R^{2} \int_{-1}^{0}\left(r^{2}-e_{u s}^{2}-2 \beta_{p} e_{u s} r\right) \mathrm{d} r \Omega^{2} \beta_{T}+R^{2} \int_{-1}^{0}-r\left(\beta_{p} r+e_{u s}\right) \mathrm{d} r \Omega^{2} \\
& \quad=-R \int_{0}^{1} F_{r}\left(\left(\beta_{T}+\beta_{p}\right) r-e_{u s}\right) \mathrm{d} r-R \int_{-1}^{0} F_{r}\left(\left(\beta_{T}-\beta_{p}\right) r-e_{u s}\right) \mathrm{d} r \\
& \quad+R \int_{0}^{1} F_{z}\left(r-\left(\beta_{p} r-e_{u s}\right) \beta_{T}\right) \mathrm{d} r+R \int_{-1}^{0} F_{z}\left(r+\left(\beta_{p} r+e_{u s}\right) \beta_{T}\right) \mathrm{d} r \\
& R^{2} \int_{e_{l l}}^{1} m_{d}\left(r-e_{l l}\right)^{2} \mathrm{~d} r \ddot{\zeta} \\
& \quad+2 R^{2} \int_{e_{l l}}^{1} m_{d} r\left(r-e_{l l}\right) \mathrm{d} r \beta_{T} \Omega \dot{\beta}_{T}+2 R^{2} \int_{e_{l l}}^{1} m_{d}\left(r-e_{l l}\right)\left(\beta_{p} r-e_{u s}\right) \mathrm{d} r \Omega \dot{\beta}_{T} \\
& +R^{2} e_{l l} \int_{e_{l l}}^{1} m_{d}\left(r-e_{l l}\right) \mathrm{d} r \Omega^{2} \zeta-R^{2} \int_{e_{l l}}^{1} m_{d}\left(r-e_{l l}\right)\left(2 \beta_{p} r-e_{u s}\right) \mathrm{d} r \beta_{T} \Omega^{2} \zeta \\
& \quad=R \int_{e_{l l}}^{1}\left(F_{x}-F_{r} \zeta\right)\left(r-e_{l l}\right) \mathrm{d} r
\end{aligned}
$$


Collecting inertia, Coriolis and centrifugal terms and changing the integration variable of the opposite blade from $r$ to $-r$ allows evaluation of the moments of inertia, the non-dimensional natural frequencies and the integrals of the Coriolis terms as shown below. Note that the Coriolis acceleration terms are divided into two parts and that $I_{\mathrm{Cor}_{2}}$ is independent of teetering deflection $\beta_{T}$.

$$
\begin{gathered}
I_{\beta_{T}}=2 R^{2} \int_{0}^{1} m_{d}\left(r^{2}+e_{u s}^{2}-2 \beta_{p} e_{u s} r\right) \mathrm{d} r \\
I_{\zeta}=R^{2} \int_{e_{l l}}^{1} m_{d}\left(r-e_{l l}\right)^{2} \mathrm{~d} r \\
v_{\beta_{T}}^{2}=1+\frac{2 R^{2}}{I_{\beta_{T}}} \int_{0}^{1} m_{d}\left(-2 e_{u s}^{2}+4 \beta_{p} e_{u s} r\right) \mathrm{d} r \\
I_{\zeta}\left(e_{l l} \int_{e_{l l}}^{1} m_{d}\left(r-e_{l l}\right) \mathrm{d} r-\int_{e_{l l}}^{1} m_{d}\left(r-e_{l l}\right)\left(2 \beta_{p} r-e_{u s}\right) \mathrm{d} r \beta_{T}\right) \\
I_{C o r_{1}}=R^{2} \int_{e_{l l}}^{1} m_{d} r\left(r-e_{l l}\right) \mathrm{d} r \\
I_{C o r_{2}}=R^{2} \int_{e_{l l}}^{1} m_{d}\left(r-e_{l l}\right)\left(\beta_{p} r-e_{u s}\right) \mathrm{d} r
\end{gathered}
$$

Substituting Equations C.64 to C.69 into Equations C.62 and C.63 and performing the same variable substitution on the force terms acting on the opposite blade allows the equations of motion for the teetering rotor hub to be written as shown in Equations C.70 and C.71.

$$
\begin{aligned}
I_{\beta_{T}}\left(\ddot{\beta}_{T}+v_{\beta_{T}}^{2} \Omega^{2} \beta_{T}-2 \frac{I_{C o r_{1}}}{I_{\beta_{T}}} \beta_{T} \Omega\left(\dot{\zeta}_{\psi}+\dot{\zeta}_{\psi+\pi}\right)-2 \frac{I_{C o r_{2}}}{I_{\beta_{T}}} \Omega\left(\dot{\zeta}_{\psi}-\dot{\zeta}_{\psi+\pi}\right)\right) \\
=-R \int_{0}^{1} F_{r_{\psi}}\left(\left(\beta_{T}+\beta_{p}\right) r-e_{u s}\right) \mathrm{d} r-R \int_{0}^{1} F_{r_{\psi+\pi}}\left(\left(\beta_{T}-\beta_{p}\right)(-r)-e_{u s}\right) \mathrm{d} r \\
\quad+R \int_{0}^{1} F_{z_{\psi}}\left(r-\left(\beta_{p} r-e_{u s}\right) \beta_{T}\right) \mathrm{d} r+R \int_{0}^{1}-F_{z_{\psi+\pi}}\left(r+\left(\beta_{p} r-e_{u s}\right) \beta_{T}\right) \mathrm{d} r
\end{aligned}
$$


APPENDIX C. BLADE DYNAMICS - EQUATIONS OF MOTION

$$
I_{\zeta}\left(\ddot{\zeta}+v_{\zeta}^{2} \Omega^{2} \zeta+2 \frac{I_{C_{C o r}} \beta_{T}+I_{C o r_{2}}}{I_{\zeta}} \Omega \dot{\beta}_{T}\right)=R \int_{e_{l l}}^{1}\left(F_{x}-F_{r} \zeta\right)\left(r-e_{l l}\right) \mathrm{d} r
$$

Velocity and acceleration terms are non-dimensionalized and the equations of motion are solved for teetering and lead-lag acceleration, respectively, to form Equations C.72 and C.73 .

$$
\begin{aligned}
\beta_{T}^{* *}=-v_{\beta_{T}}^{2} \beta_{T}+2 \frac{I_{C o r_{1}}}{I_{\beta_{T}}} \beta_{T}\left(\zeta_{\psi}^{*}+\zeta_{\psi+\pi}^{*}\right)+2 \frac{I_{C o r_{2}}}{I_{\beta_{T}}}\left(\zeta_{\psi}^{*}-\zeta_{\psi+\pi}^{*}\right) \\
+\frac{R}{I_{\beta_{T}} \Omega^{2}}\left[\int_{0}^{1} F_{z_{\psi}}\left(r-\left(\beta_{p} r-e_{u s}\right) \beta_{T}\right) \mathrm{d} r-\int_{0}^{1} F_{z_{\psi+\pi}}\left(r+\left(\beta_{p} r-e_{u s}\right) \beta_{T}\right) \mathrm{d} r\right. \\
\left.-\int_{0}^{1} F_{r_{\psi}}\left(\left(\beta_{T}+\beta_{p}\right) r-e_{u s}\right) \mathrm{d} r+\int_{0}^{1} F_{r_{\psi+\pi}}\left(\left(\beta_{T}-\beta_{p}\right) r+e_{u s}\right) \mathrm{d} r\right] \\
\zeta^{* *}=-v_{\zeta}^{2} \zeta-2 \frac{I_{C o r_{1}} \beta_{T}+I_{C o r_{2}}}{I_{\zeta}} \beta_{T}^{*}+\frac{R}{I_{\zeta} \Omega^{2}} \int_{e_{l l}}^{1}\left(r-e_{l l}\right)\left(F_{x}-F_{r} \zeta\right) \mathrm{d} r
\end{aligned}
$$

To model a teetering rotor without lead-lag hinges, Equation C.72 may be reduced to form Equation C.74. Equation C.73 trivially disappears.

$$
\begin{gathered}
\beta_{T}^{* *}=-v_{\beta_{T}}^{2} \beta_{T}+\frac{R}{I_{\beta_{T}} \Omega^{2}}\left[\int_{0}^{1} F_{z_{\psi}}\left(r-\left(\beta_{p} r-e_{u s}\right) \beta_{T}\right) \mathrm{d} r-\int_{0}^{1} F_{z_{\psi+\pi}}\left(r+\left(\beta_{p} r-e_{u s}\right) \beta_{T}\right) \mathrm{d} r\right. \\
\left.-\int_{0}^{1} F_{r_{\psi}}\left(\left(\beta_{T}+\beta_{p}\right) r-e_{u s}\right) \mathrm{d} r+\int_{0}^{1} F_{r_{\psi+\pi}}\left(\left(\beta_{T}-\beta_{p}\right) r+e_{u s}\right) \mathrm{d} r\right]
\end{gathered}
$$

\section{Addition of a Teetering Hinge Spring and a Lead-lag Hinge Damper}

Teetering rotor hubs may feature a teetering spring and while full-sized teetering rotors in general do not have lead-lag hinges and hence no need for lead-lag dampers dynamic damping was also included in the equations implemented in the forward flight module. This was done since $\mathrm{r} / \mathrm{c}$ helicopter lead-lag motion occurs about a clamped pin attachment point instead of a bearing and the resulting frictional resistance at the joint is expected to have some lead-lag damping effect. The implementation of these two components into the equations of motion is very similar to the derivation outlined 
above for the fully articulated rotor. Therefore only a few notes regarding significant differences are provided here.

The teetering hinge spring is defined to have a spring stiffness $K_{\beta}$ - in units of torque per radian - and neutral position at $\beta_{T}=0$. This results in a change to the non-dimensional natural teetering frequency $v_{\beta_{T}}$ as shown in Equation C.75. The teetering and lead-lag equations of motion are not further affected by the addition of the spring.

$$
v_{\beta_{T}}^{2}=1+\frac{2 R^{2}}{I_{\beta_{T}}} \int_{0}^{1} m_{d}\left(-2 e_{u s}^{2}+4 \beta_{p} e_{u s} r\right) \mathrm{d} r+\frac{K_{\beta_{T}}}{I_{\beta_{T}} \Omega^{2}}
$$

The lead-lag damper is implemented in the same fashion as shown for the fully articulated rotor above. The final lead-lag equation of motion solved for the nondimensional lead-lag acceleration gains a term to account for the effect of the damper as shown in Equation C.76.

$$
\zeta^{* *}=-v_{\zeta}^{2} \zeta-2 \frac{I_{C o r_{1}} \beta_{T}+I_{C o r_{2}}}{I_{\zeta}} \beta_{T}^{*}-\frac{C_{\zeta}}{I_{\zeta} \Omega} \zeta^{*}+\frac{R}{I_{\zeta} \Omega^{2}} \int_{e_{l l}}^{1}\left(r-e_{l l}\right)\left(F_{x}-F_{r} \zeta\right) \mathrm{d} r
$$

\section{Discussion on Alternative Rotor Hub Designs}

This work assumes that the $\mathrm{r} / \mathrm{c}$ helicopter rotor under consideration features a twobladed teetering rotor hub, which is a common implementation used for example on the baseline ING Robotic Aviation Responder UAS. However, it has come to the attention of the author that another common type of $\mathrm{r} / \mathrm{c}$ helicopter rotor hubs - for two or more bladed rotors - does not include a teetering or flapping hinge at all ${ }^{4}$. Such a rigid rotor design on full sized helicopters usually depends on rotor articulation about flexible hub or blade components near the axis of rotation and improves the rotor response to cyclic

\footnotetext{
${ }^{4}$ Lead-lag motion is still possible about the blade attachment bolt.
} 
control inputs. Some r/c implementations, however, use very stiff rotor hubs often manufactured from solid aluminum. While no specific reference documentation could be located, it is the author's opinion that flapping motion for such a design heavily depends on the flexibility of the rotor blades.

Since only rigid blade motion is accounted for in the equations of motion implemented in the simulation algorithm, analysis of the forward flight performance of such a rigid rotor is not directly possible. One solution would be to implement flexible blade structures using for example a beam model to represent elastic deformations. Another possibility would be to simulate such a rotor as a fully articulated rotor and use an equivalent hinge location, flapping hinge spring stiffness and blade flapping inertia that approximate the flapping motion that occurs due to blade flexibility. The latter option is fully implementable using the current form of the equations of motion. 


\section{Appendix D}

\section{Additional Forward Flight Module Validation Figures and Discussion}

This appendix contains additional validation figures for the forward flight module. Experimental results are taken from Reference [67], which considers a scaled $\mathrm{CH}-47 \mathrm{C}$ rotor model operating in the "aft-rotor-alone" configuration with the forward rotor removed. Reference [67] provides data for three parameter sweeps, which include the advance ratio sweep already discussed in Section 3.3.1, a collective pitch sweep and a shaft angle of attack sweep. Comparisons of the output from the forward flight module to the experimental data of the latter two cases are presented here.

Rotor parameters are listed in Table D.1. Blade mass was estimated from the weight moment and moment of inertia parameters assuming a linear spanwise mass distribution. The location of the lead-lag hinge was assumed to coincide with that of the flapping hinge. XFOIL was used to generate low Reynolds number airfoil coefficients for the V23010-1.58 airfoil. Airfoil profile coordinates ${ }^{1}$ were obtained from Reference [63].

\footnotetext{
${ }^{1}$ Trailing edge tab deflection was assumed to be $0^{\circ}$.
} 
Table D.1: Scaled CH-47C rotor characteristics and test condition [67].

\begin{tabular}{cc}
\hline Parameter & Baseline \\
\hline \hline Number of Blades & 4 \\
Diameter (m) & 1.66 \\
Rotor Speed (rpm) & 1574 \\
Tip Speed (m/s) & 137.2 \\
Rotor Solidity & 0.0892 \\
Twist $\left(^{\circ}\right)$ & -9.14 \\
Planform & Rectangular \\
Airfoil & V23010-1.58 \\
Chord Length (m) & 0.0582 \\
Flapping Hinge Offset (m) & 0.0191 \\
Weight Moment (N m) & 0.664 \\
Moment of Inertia (kg m $\left.{ }^{2}\right)$ & 0.0366 \\
Blade Mass (kg) & 0.195 \\
\hline
\end{tabular}

The collective sweep was performed at a constant advance ratio of 0.08 and approximately constant shaft angle of attack. Due to the offset in collective pitch observed during the simulation of the advance ratio sweep, the iteration goal of the simulation was chosen to be the thrust coefficient along with advance ratio and disk angle of attack as other input parameters. The forward flight module collective sweep results are shown in Figure D.1.

Overall trends with respect to inflow model selection observed were very similar to those already discussed for the advance ratio sweep in Section 3.3.1. Out of the linear inflow models the Drees model reproduced the longitudinal rotor parameters best, while the Pitt \& Peters model showed best agreement for the lateral parameters. The offset in collective pitch was again observed relatively constant over the whole 
collective sweep range. However, the error in power coefficient increased with increasing collective pitch.
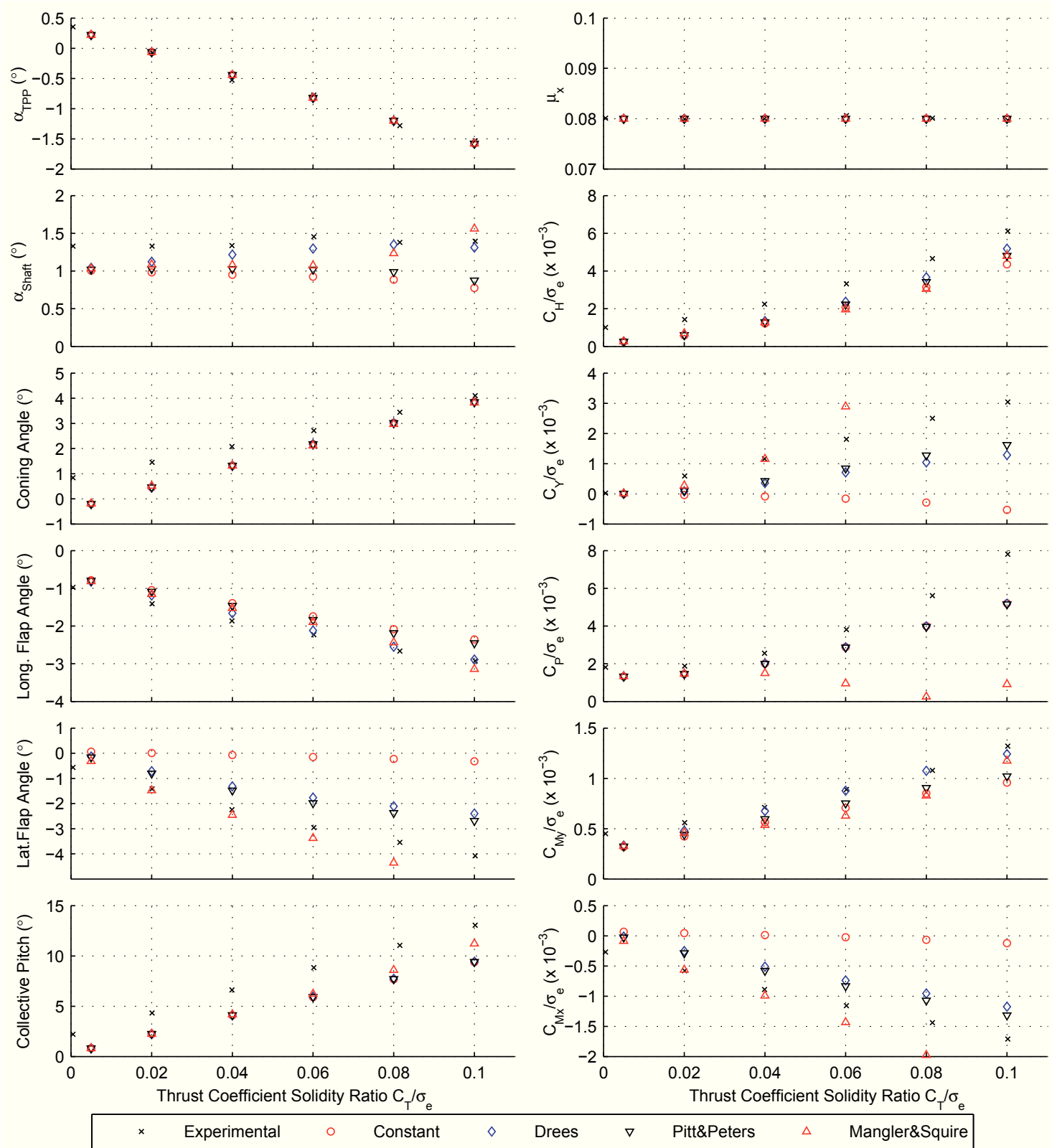

Figure D.1: Comparison of forward flight module simulated results to experimental data from Reference [67]: collective sweep. 
The third parameter sweep was with respect to shaft angle of attack, which varied from about $2^{\circ}$ backward tilt to $6^{\circ}$ forward tilt. Thrust coefficient, disk angle of attack and advance ratio were chosen as inputs to the simulation.

As shown in Figure D.2 most parameters remained relatively constant, though small gradients with respect to shaft angle of attack were reproduced well. The linear Drees and Pitt \& Peters inflow models provided best matches to the longitudinal and lateral parameters, respectively. A uniform offset was observed for collective pitch and power coefficient.

As the advance ratio for the collective and shaft angle of attack sweeps is held constant at 0.08 -i.e. below 0.1 - the Mangler \& Squire inflow model's inferior performance was not surprising. Similarly, since the advance ratio of 0.08 represented the condition of greatest lateral effect for the test rotor, the constant inflow model was not expected to provide representative results. 

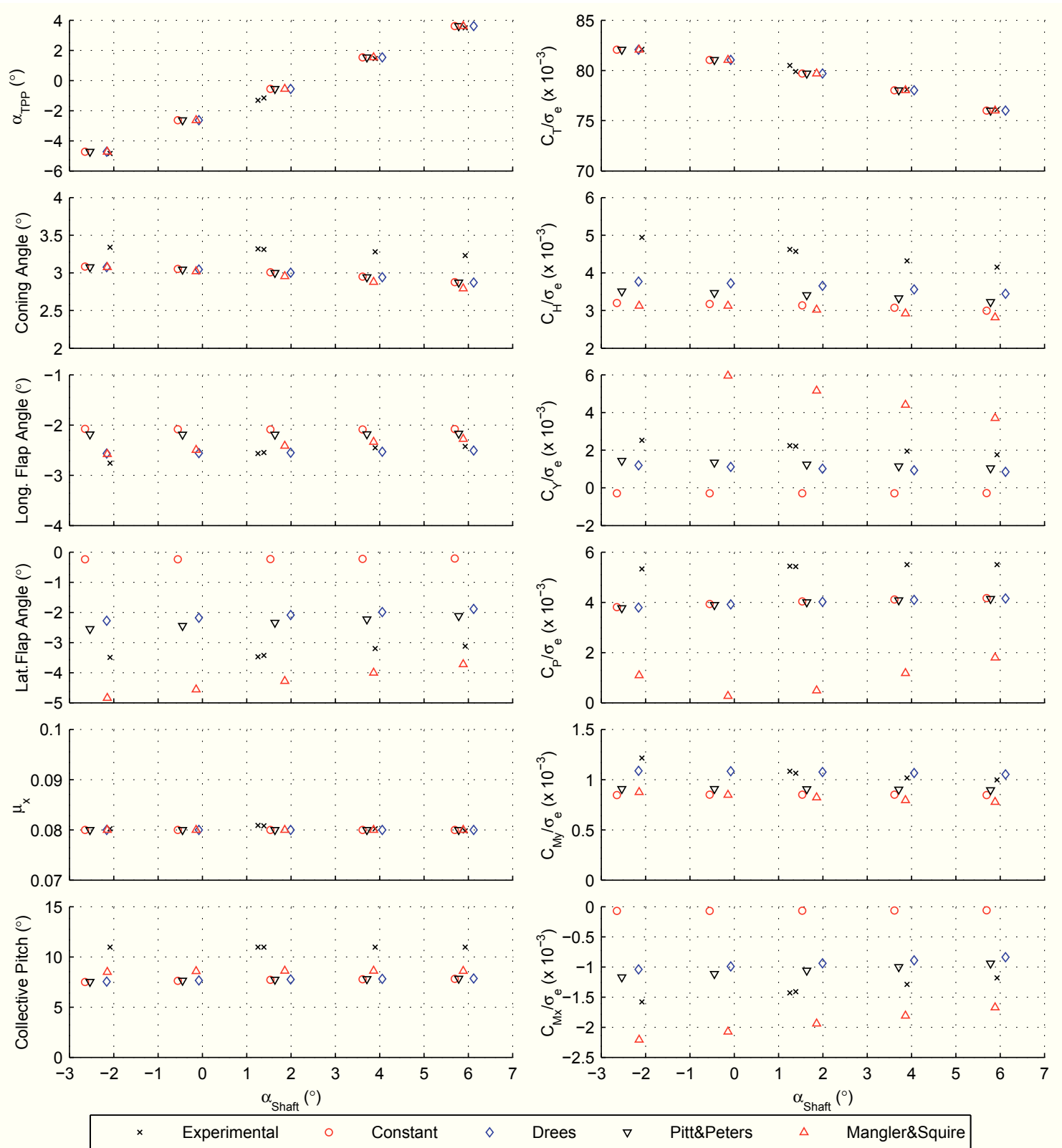

Figure D.2: Comparison of forward flight module simulated results to experimental data from Reference [67]: shaft angle of attack sweep. 


\section{Appendix E}

\section{Selected Rotorcraft Airfoils Evaluated at Reynolds Numbers Between 100,000 and $1,000,000$}

A number of airfoils developed for use on rotorcraft blades were evaluated at various Reynolds numbers using XFOIL [48] to determine their applicability in the Reynolds number range expected for a 700 class electric $r / c$ helicopter main rotor. Airfoil parameters were calculated in angle of attack increments of $0.5^{\circ}$ assuming incompressible flow and using transition parameter $n_{\text {crit }}=9$. Parameters at the maximum lift coefficient $C_{l_{\max }}$ and maximum airfoil lift to drag ratio $\left(C_{l} / C_{d}\right)_{\max }$ are listed in Tables E.1 to E.4. Airfoil profile coordinates were obtained from References [63] and [22]. 
Table E.1: Selected rotorcraft airfoil parameters at $\mathrm{Re}=100,000$.

\begin{tabular}{crrrr|rrrr}
\hline Airfoil & $C_{l_{\max }}$ & $\alpha$ & $\frac{C_{l}}{C_{d}}$ & $C_{m}$ & $\left(\frac{C_{l}}{C_{d}}\right)_{\max }$ & $\alpha$ & $C_{l}$ & $C_{m}$ \\
\hline \hline NACA 0009 & 0.82 & 8.5 & 15.4 & 0.0176 & 35.5 & 4.0 & 0.48 & -0.0047 \\
NACA 0012 & 0.97 & 10.0 & 21.1 & 0.0217 & 36.7 & 5.0 & 0.61 & -0.0077 \\
NACA 0015 & 1.11 & 12.0 & 23.4 & 0.0306 & 37.4 & 6.0 & 0.76 & -0.0130 \\
NACA 23012 & 1.25 & 12.0 & 28.0 & 0.0167 & 36.4 & 5.5 & 0.77 & -0.0119 \\
NACA 23015 & 1.24 & 12.0 & 26.5 & 0.0198 & 36.1 & 5.5 & 0.82 & -0.0188 \\
V23010-1.58 w/ 0 TE tab & 1.32 & 14.0 & 25.7 & 0.0058 & 37.5 & 10.5 & 1.13 & -0.0053 \\
V43012-1.58 w/ 0 TE tab & 1.11 & 9.0 & 23.0 & -0.0036 & 33.1 & 5.5 & 0.79 & 0.0041 \\
NACA 8-H-012 & 1.19 & 14.5 & 16.0 & 0.0223 & 44.8 & 9.5 & 1.18 & -0.0053 \\
VR-7 w/ 0 TE tab & 1.35 & 13.5 & 21.5 & -0.0126 & 51.8 & 9.0 & 1.21 & -0.0324 \\
VR-8 w/ 0 ${ }^{\circ}$ TE tab & 0.92 & 8.0 & 18.6 & -0.0055 & 44.9 & 5.0 & 0.64 & -0.0106 \\
RC(3)-08 & 0.89 & 8.5 & 17.3 & 0.0120 & 45.3 & 4.5 & 0.63 & -0.0180 \\
RC(3)-10 & 1.09 & 10.5 & 20.7 & 0.0128 & 49.0 & 5.0 & 0.76 & -0.0303 \\
RC(4)-10 & 1.15 & 11.0 & 20.6 & 0.0085 & 37.2 & 3.5 & 0.56 & -0.0148 \\
FX 69-H-098 & 1.17 & 12.0 & 24.0 & 0.0173 & 44.0 & 8.5 & 1.04 & -0.0108 \\
\hline
\end{tabular}


Table E.2: Selected rotorcraft airfoil parameters at $\mathrm{Re}=200,000$.

\begin{tabular}{crrrr|rrrr}
\hline Airfoil & $C_{l_{\max }}$ & $\alpha$ & $\frac{C_{l}}{C_{d}}$ & $C_{m}$ & $\left(\frac{C_{l}}{C_{d}}\right)_{\max }$ & $\alpha$ & $C_{l}$ & $C_{m}$ \\
\hline \hline NACA 0009 & 0.87 & 9.0 & 19.6 & 0.0172 & 43.5 & 4.0 & 0.48 & -0.0045 \\
NACA 0012 & 1.11 & 12.5 & 22.8 & 0.0327 & 47.4 & 5.0 & 0.62 & -0.0099 \\
NACA 0015 & 1.20 & 15.0 & 23.3 & 0.0461 & 49.5 & 6.0 & 0.77 & -0.0162 \\
NACA 23012 & 1.32 & 14.0 & 31.6 & 0.0249 & 51.9 & 9.0 & 1.08 & -0.0005 \\
NACA 23015 & 1.36 & 16.0 & 25.3 & 0.0346 & 50.2 & 7.5 & 0.98 & -0.0088 \\
V23010-1.58 w/ 0 ${ }^{\circ}$ TE tab & 1.37 & 14.0 & 35.3 & -0.0008 & 57.4 & 9.5 & 1.05 & -0.0094 \\
V43012-1.58 w/ 0 ${ }^{\circ}$ TE tab & 1.57 & 14.0 & 39.0 & 0.0018 & 48.6 & 7.5 & 1.02 & 0.0016 \\
NACA 8-H-012 & 1.21 & 15.5 & 16.2 & 0.0193 & 71.1 & 9.0 & 1.17 & -0.0071 \\
VR-7 w/ 0 ${ }^{\circ}$ TE tab & 1.34 & 14.5 & 20.1 & -0.0227 & 72.9 & 8.0 & 1.13 & -0.0338 \\
VR-8 w/ 0 ${ }^{\circ}$ TE tab & 0.93 & 8.5 & 15.9 & -0.0070 & 56.3 & 4.5 & 0.58 & -0.0092 \\
RC(3)-08 & 0.91 & 8.5 & 23.6 & 0.0073 & 56.7 & 3.5 & 0.54 & -0.0234 \\
RC(3)-10 & 1.15 & 11.5 & 24.8 & 0.0183 & 62.8 & 4.5 & 0.71 & -0.0315 \\
RC(4)-10 & 1.40 & 14.0 & 33.1 & 0.0191 & 47.0 & 2.5 & 0.47 & -0.0182 \\
FX 69-H-098 & 1.18 & 11.5 & 36.0 & 0.0111 & 60.1 & 8.0 & 1.01 & -0.0151 \\
\hline
\end{tabular}


Table E.3: Selected rotorcraft airfoil parameters at $\mathrm{Re}=500,000$.

\begin{tabular}{crrrr|rrrr}
\hline Airfoil & $C_{l_{\max }}$ & $\alpha$ & $\frac{C_{l}}{C_{d}}$ & $C_{m}$ & $\left(\frac{C_{l}}{C_{d}}\right)_{\max }$ & $\alpha$ & $C_{l}$ & $C_{m}$ \\
\hline \hline NACA 0009 & 1.04 & 11.0 & 26.9 & 0.0202 & 53.5 & 5.5 & 0.62 & -0.0019 \\
NACA 0012 & 1.24 & 15.0 & 25.8 & 0.0334 & 61.8 & 6.0 & 0.72 & -0.0100 \\
NACA 0015 & 1.28 & 16.0 & 25.7 & 0.0362 & 66.5 & 7.5 & 0.91 & -0.0140 \\
NACA 23012 & 1.45 & 14.5 & 44.7 & 0.0201 & 77.8 & 8.5 & 1.06 & -0.0067 \\
NACA 23015 & 1.45 & 16.0 & 32.4 & 0.0263 & 73.7 & 9.0 & 1.12 & -0.0049 \\
V23010-1.58 w/ 0 TE tab & 1.48 & 15.0 & 39.9 & -0.0045 & 79.4 & 8.5 & 0.96 & -0.0128 \\
V43012-1.58 w/ 0 TE tab & 1.63 & 13.5 & 62.6 & -0.0058 & 78.7 & 10.0 & 1.31 & -0.0031 \\
NACA 8-H-012 & 1.23 & 17.5 & 12.9 & 0.0034 & 103.1 & 7.5 & 1.04 & -0.0090 \\
VR-7 w/ 0 TE tab & 1.44 & 13.5 & 32.8 & -0.0331 & 99.9 & 7.0 & 1.02 & -0.0337 \\
VR-8 w/ 0 ${ }^{\circ}$ TE tab & 1.02 & 9.0 & 24.7 & -0.0123 & 69.3 & 4.0 & 0.52 & -0.0093 \\
RC(3)-08 & 1.05 & 10.5 & 29.5 & 0.0164 & 67.6 & 3.0 & 0.48 & -0.0225 \\
RC(3)-10 & 1.22 & 13.5 & 32.5 & 0.0347 & 74.3 & 3.5 & 0.62 & -0.0342 \\
RC(4)-10 & 1.55 & 16.0 & 39.3 & 0.0160 & 69.7 & 8.0 & 1.00 & -0.0057 \\
FX 69-H-098 & 1.32 & 13.0 & 43.6 & 0.0116 & 79.1 & 6.0 & 0.84 & -0.0255 \\
\hline
\end{tabular}


Table E.4: Selected rotorcraft airfoil parameters at $\operatorname{Re}=1,000,000$.

\begin{tabular}{crrrr|rrrr}
\hline Airfoil & $C_{l_{\max }}$ & $\alpha$ & $\frac{C_{l}}{C_{d}}$ & $C_{m}$ & $\left(\frac{C_{l}}{C_{d}}\right)_{\max }$ & $\alpha$ & $C_{l}$ & $C_{m}$ \\
\hline \hline NACA 0009 & 1.23 & 13.5 & 33.1 & 0.0252 & 67.7 & 7.0 & 0.78 & 0.0003 \\
NACA 0012 & 1.39 & 15.5 & 39.0 & 0.0315 & 75.6 & 7.5 & 0.87 & -0.0067 \\
NACA 0015 & 1.42 & 17.0 & 31.8 & 0.0345 & 78.9 & 9.0 & 1.04 & -0.0069 \\
NACA 23012 & 1.49 & 15.0 & 46.1 & 0.0196 & 95.6 & 8.0 & 1.04 & -0.0119 \\
NACA 23015 & 1.59 & 17.0 & 39.1 & 0.0249 & 95.3 & 9.0 & 1.17 & -0.0134 \\
V23010-1.58 w/ 0 ${ }^{\circ}$ TE tab & 1.64 & 16.5 & 46.2 & -0.0039 & 90.6 & 7.5 & 0.85 & -0.0133 \\
V43012-1.58 w/ 0 ${ }^{\circ}$ TE tab & 1.61 & 14.0 & 49.3 & -0.0061 & 100.4 & 8.5 & 1.15 & -0.0008 \\
NACA 8-H-012 & 1.28 & 16.0 & 17.6 & 0.0025 & 125.0 & 6.5 & 0.94 & -0.0091 \\
VR-7 w/ 0 ${ }^{\circ}$ TE tab & 1.57 & 15.0 & 33.3 & -0.0350 & 118.3 & 6.5 & 0.97 & -0.0332 \\
VR-8 w/ 0 ${ }^{\circ}$ TE tab & 1.09 & 9.5 & 30.8 & -0.0147 & 72.9 & 6.5 & 0.82 & -0.0169 \\
RC(3)-08 & 1.24 & 13.0 & 39.1 & 0.0245 & 71.5 & 6.0 & 0.78 & -0.0187 \\
RC(3)-10 & 1.38 & 15.0 & 42.0 & 0.0330 & 79.0 & 7.0 & 0.96 & -0.0288 \\
RC(4)-10 & 1.64 & 17.0 & 41.7 & 0.0156 & 92.0 & 9.0 & 1.12 & -0.0065 \\
FX 69-H-098 & 1.40 & 14.0 & 45.1 & 0.0133 & 91.4 & 5.5 & 0.80 & -0.0296 \\
\hline
\end{tabular}

